\title{
Ocean, Platform, and Signal Processing Effects on Synthetic Aperture Sonar Performance
}

\author{
by \\ Kenneth D. Rolt

\begin{abstract}
Submitted in partial fulfillment
of the requirements for the degree of

Scientiae Magister
\end{abstract} \\ at the \\ Massachusetts Institute of Technology \\ Cambridge, MA 02139 \\ February 1991
}

(C) Kenneth D. Rolt, 1990; All rights reserved. The author hereby grants MIT, C.S. Draper Labs, and the Raytheon Company, permission to reproduce and/or distribute copies of this thesis in-whole or in-part.

Signature of Author

Department of Ocean Engineering

28 November 1990

Certified by

Professor Jerome Milgram

Thesis Co-Supervisor

Certified by

Professor Henrik Schmidt

Thesis Co-Supervisor

Accepted by

Professor A. Douglas Carmichael

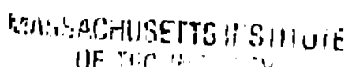
Ark 05 1991

LuErimatics 


\title{
Ocean, Platform, and Signal Processing Effects on Synthetic Aperture Sonar Performance
}

\author{
by Kenneth D. Rolt \\ keywords: synthetic aperture somar (SAS), synthetic aperture radar (SAR), \\ acoustical imaging, radar imaging
}

\begin{abstract}
Synthetic Aperture Sonar (SAS), the underwater sound application of the aperture synthesis method borrowed from Synthetic Aperture Radar (SAR), is investigated by means of ocean medium effects, platform motion effects, and signal processing effects. Synthetic aperture methods, both in radar and in sonar, allow large synthesized apertures, or equivalently synthesized arrays, to be formed from the combined use of signal processing and a small, real aperture transducer (e.g. a radar or a sonar transducer). Large synthetic apertures give rise to relatively narrow synthesized beams, which in turn allow very high resolution focused images to be formed from radar or sonar data. The target in the synthetic aperture sonar case could be the ocean bottom, and the objective could be, for example, ocean-bottom mapping or searching for a sunken vessel.
\end{abstract}

While synthetic aperture radars have been extensively developed and used since the late 1959's, synthetic aperture sonars have been rare except for a number of academic papers and a few experimental systems. A computer-based model has been created to evaluate the imaging potential of a synthetic aperture sonar system within a horizontally stratified ocean. The model includes ocean refraction, spatial and temporal coherence, surface and bottom influences via multipath, deterministic and random platform motion, and a variety of processing options. This model, the most extensive ever used for SAS simulations, has been verified by comparison to a SAS ocean experiment performed by others. By the use of the model, the three dominant influences of ocean, platform, and signal processing may be studied on the performance of synthetic aperture sonar imaging.

The ocean influence on SAS imagery is shown to depend on (1) the extent of spatial/temporal coherence which limits the useful aperture length that may be used for sharp imaging, and (2) the refraction profile which must be accounted for in image reconstruction to ensure a match between the real ocean and the estimated ocean in the computer model; this turns out to be a geometric matched filter. 
Platform motion is also shown to degrade the imagery, and the model confirms that lateral platform motion should be measured to within the canonical $\lambda / 8$ distance found in the SAR literature for the sharpest images, but also that the image persists even for lateral platform motion in excess of $\lambda / 8$.

Finally, a number of processing effects are shown, with the most important being that: (1) synthetic aperture radars and sonars working exactly at the Nyquist sampling rate do not entirely null alias lobes (an assumption often made in the SAS literature); (2) the use of many sub-aperture lengths for a Nyquist sampled synthetic aperture will force the aliases to the level of the far sidelobes (hence making them invisible); (3) the bandwidth must be carefully chosen for a Nyquist-sampled synthetic aperture to minimize alias images; and (4) broadband operation is always useful for undersampled synthetic apertures, and a "ballpark" formula is developed to estimate the level of alias image smear. [Work supported by the Charles Stark Draper Laboratory]. 


\section{ACKNOWLEDGEMENTS}

First, I acknowledge the guidance and support of my co-supervisors in this effort: Professor Jerome Milgram and Professor Henrik Schmidt. I also thank the Charles Stark Draper Laboratory for sponsoring the work.

I must naturally recognize the previous work upon which this thesis in constructed, particularly by those efforts in radar and sonar wave propagation, and in synthetic aperture radar and sonar. Although the bibliography in this thesis accounts for most of the published 'prior ar' in synthetic aperture sonar, the list of contributors to the field is considerably longer. This work is, in its own way, a minor improvement on an already invented and constantly-improving wheel.

Many individuals have helped me along during this work. At the start, Dr. George M. Walsh of the Raytheon Company, Submarine Signal Division, furnished me with three volumes of Submarine Signal Division reports on synthetic aperture sonar and medium stability. Dr. William Carey also furnished copies of papers on synthetic aperture sonar, particularly on passive synthetic arrays. Dr. Shahen A. Hovanessian kindly sent me a copy of his book on synthetic arrays. I had the pleasure of meeting Dr. Philippe de Heering at the Acoustical Society of America Spring Meeting (May 1990), discussed synthetic arrays with him. de Heering also furnished me with a copy of his Ph.D. thesis on SAS. I was also fortunate in finding the Dec. $1989 \mathrm{~J}$. Acoust. Soc. Am. paper on a prototype SAS by Dr. Peter T. Gough and Dr. Michael P. Hayes, of the University of Canterbury, Christchurch, New Zealand. This publication of synthetic aperture sonar images on a known target enabled me to verify my computer model. Gough and Hayes were both kind enough to correspond with me via mail concerning their work, and also sent me a copy of Dr. Hayes' Ph.D. thesis.

Howard Zebker and W.T.K. Johnson of the Jet Propulsion Laboratory, California Institute of Technology, also provided me with SAR information and images containing azimuthal aliases, which also helped confirm my synthetic aperture model.

Locally, I was also helped by many others. At MIT I was grateful for the discussions with DJ Tang, Brian Tracey and John Halsema in particular, and by many others in the office I share. Dr. Jeff Lozow and John Irza of the Charles Stark Draper Laboratory were most helpful. Dr. John L. Butler of Image Acoustics was most helpful in discussing all sorts of acoustics, including my work, and in keeping me active in the design of underwater sound sources. 
My father, George H. Rolt, deserves some sort of award (perhaps part of my diploma) for the many discussions of my work and for requesting a rough draft of this for parental scrutiny. Parental scrutiny soon gave way to parental editing, in extremis. However, both the writer $=9 . j$ the reader of this thesis greatly benefit from the editing. Thanks Dad. Christine Coughlin also gets an award for just-plain putting up with me during 1988-1990, and sticking with me because of my "future potential."

The last note is extended to the reader of this thesis: since almost no one ever really reads these things, you deserve an award for getting at. least this far.

"Work like hell, tell everyone everything you know, close a deal with a handshake, and have fun."

- Harold E. "Doc" Edgerton, a pioneer, inventor and educator in many areas, including imaging sonar. 


\section{CONTENTS}

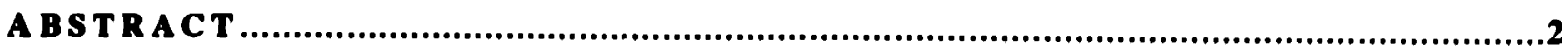

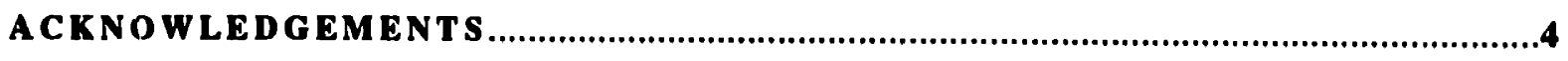

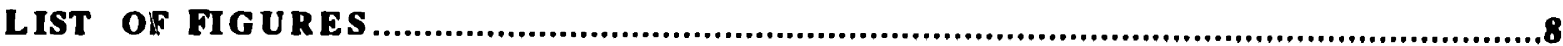

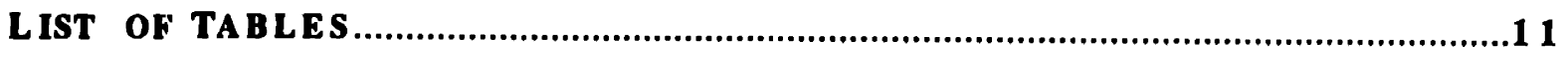

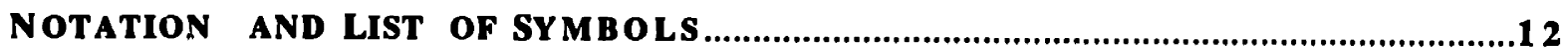

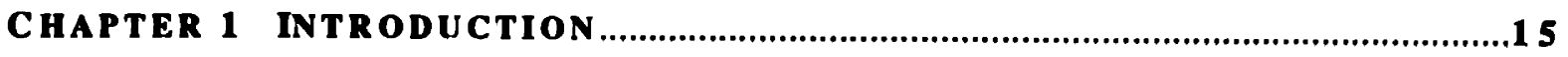

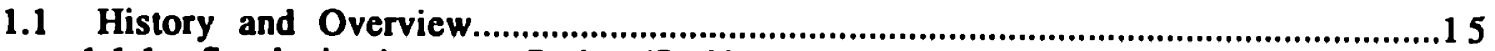

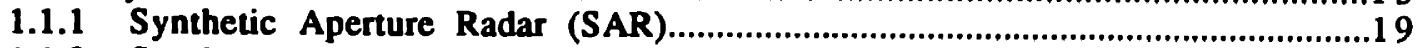

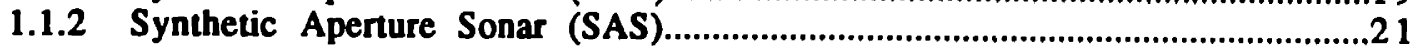

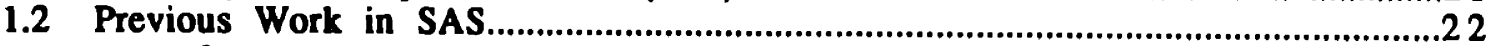

1.2.1 'Low' Frequency Active SAS (below $1 \mathrm{MHz}$ )...................................................24

1.2.2 'High' Frequency Active SAS (above $1 \mathrm{MHz}$ ).............................................28

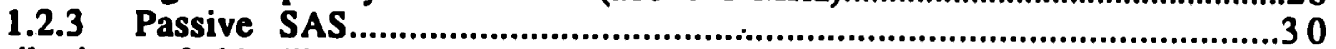

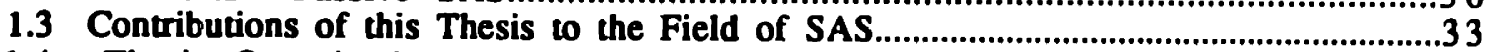

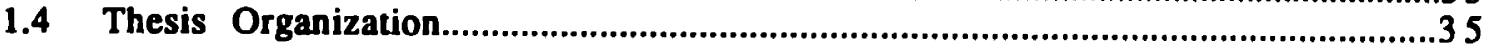

CHAPTER 2 SYNTHETIC APERTURE SONAR (SAS) PRINCIPLES..................................38

2.1 Real Apertures (Arrays) versus Synthetic Apertures (Arrays)...............................................39

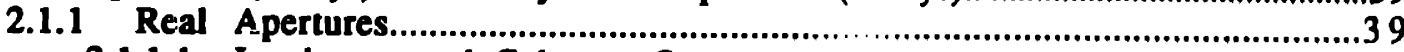

2.1.1.1 Incoherent and Coherent Sonar..............................................................46

2.1.1.2 Focused Sonar.............................................................................4

2.1.2 Synthetic Apertures (or Synthetic Arrays)...................................................47

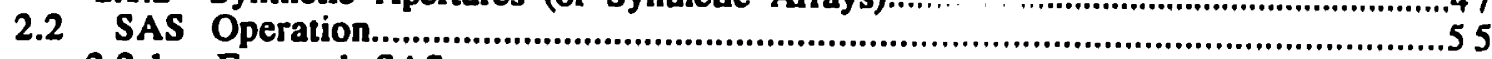

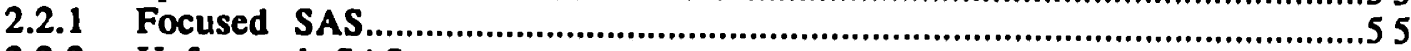

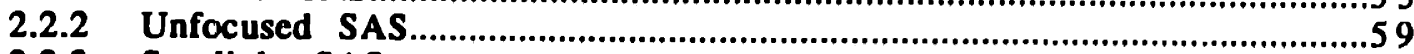

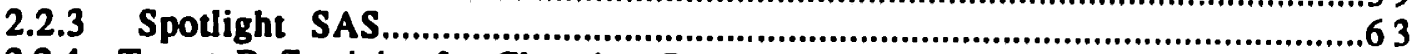

2.2.4 Target Reflectivity for Changing Sonar Positions..................................................67

2.3 Sampling Requirements and Sidelobe Limits...........................................................67

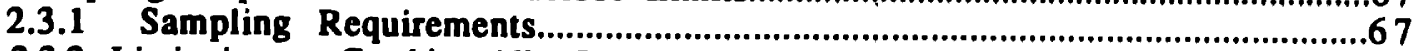

2.3.2 Limitations to Crushing Alias Lobes with the Sonar Beampattern Null..........................75

2.3.3 Vemier / Multiple-Hydrophone Approach to Sampling ..............................................84

2.3.4 PRF Requirements, Spatial Sampling, and Maximum Range.....................................86

2.3.5 Sidelobe Levels........................................................................................8 7

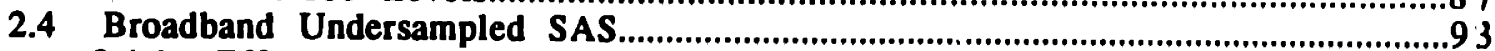

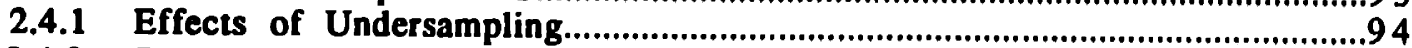

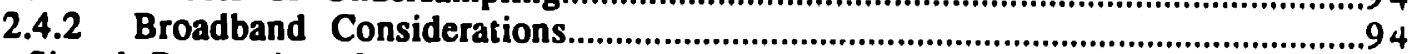

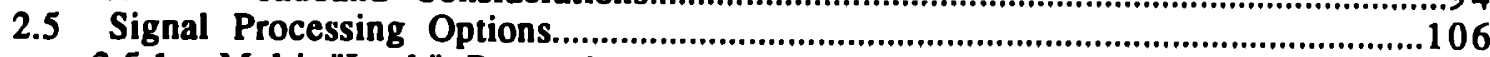

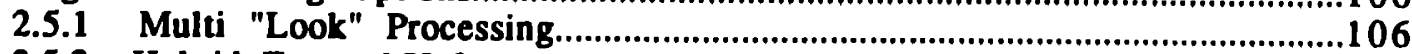

2.5.2 Hybrid Focused-Unfocused Processing...................................................................107

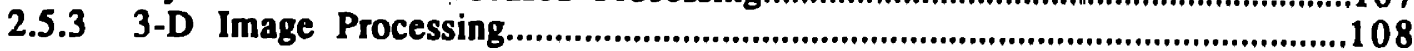


3.1 Computer Program Architecture and Processing Methods...............................................111 3.1.1 create.out

3.1.2 synth.out.

3.2 Platform/Sonar Capabilities and Assumptions.

3.3 Target, Ocean, and Boundary Properties and Assumptions.............................................116

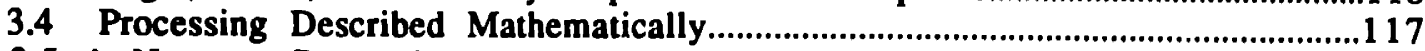

3.5 A Note on Processing.

ChaPter 4 ENVIRONMENTAL AND PLATfORM INFLUENCES ON SAS......................122

4.1 Multipath and Refraction Propagation...............................................................123

4.1.1 Isospeed Case - Surface and Bottom Effects.....................................................125

4.1.2 Deep Water Refraction Case.....................................................................126

4.2 Environment (or Medium) Stability......................................................................12

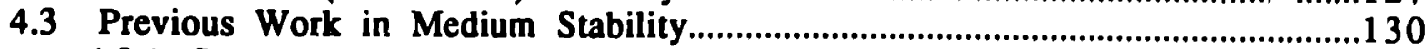

4.3.1 Summary and Comparison of Medium Stability Experiments..............................130

4.3.2 Experiment Descriptions.........................................................................13

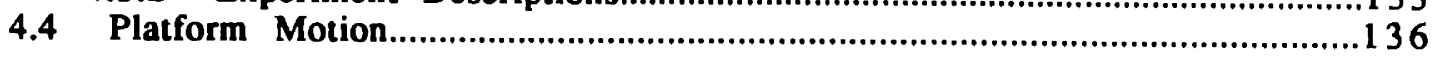

CHAPTER 5 COMPUTER MODEL EXAMPLE IMAGERY …..........................................140

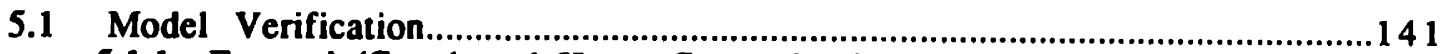

5.1.1 Focused (Gough and Hayes Comparison).......................................................141

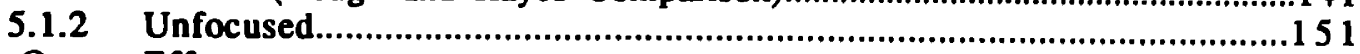

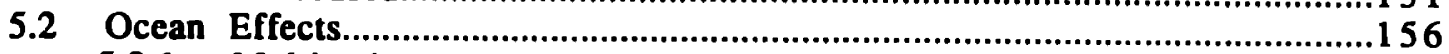

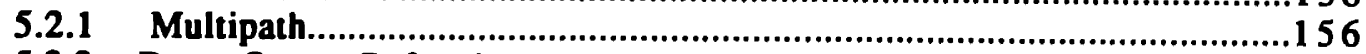

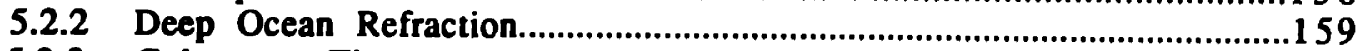

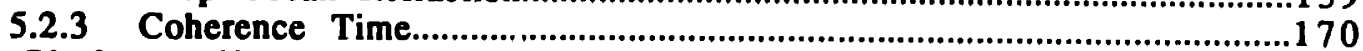

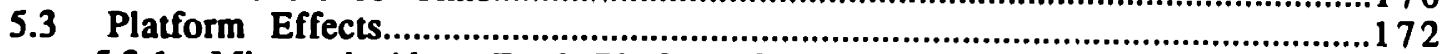

5.3.1 Mismatch Along-Track Platform Speed.........................................................1 172

5.3.2 Uncompensated Platform Motion..................................................................1 174

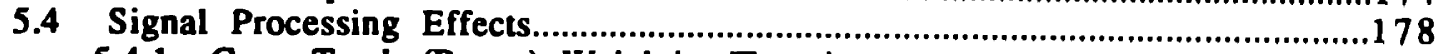

5.4.1 Cross-Track (Range) Weighting/Tapering ......................................................178

5.4.2 Along-Track (Azimuth) Weighting/Taperii!: ……..........................................178

5.4.3 Alias Levels at Nyquist Sampling Operatu(II ................................................181

5.4.4 Broadband Undersampled Operation..............................................................189

CHAPTER 6 DISCUSSION, CONCLUSIONS AND FUTURE WORK .................................196

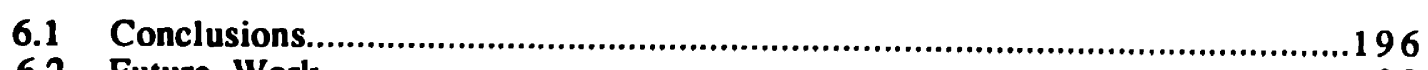

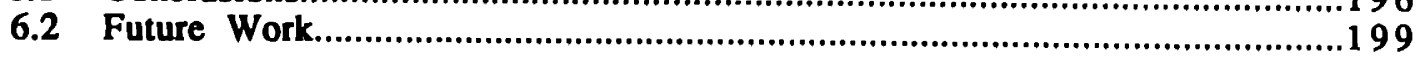

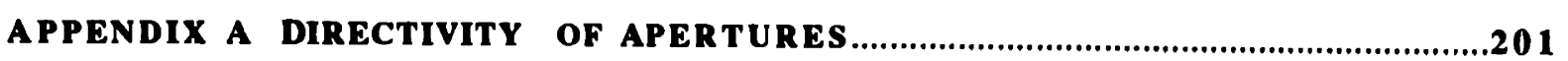

A PPENDIX B RANGE RESOLUTION AND PULSE COMPRESSION .................................207

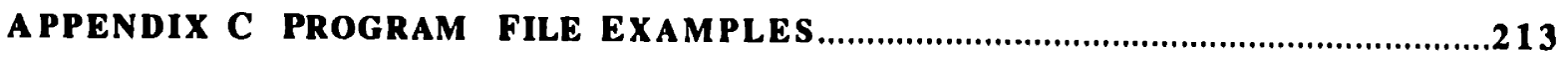

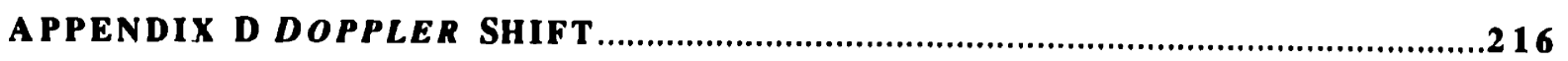

APPENDIX E DERIVE PLATFORM MOTION EQUATION...........................................222

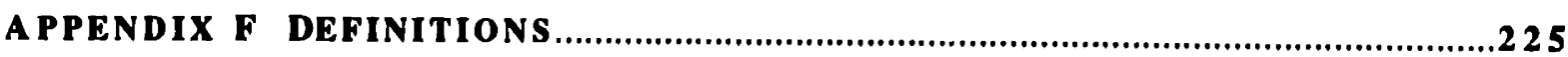

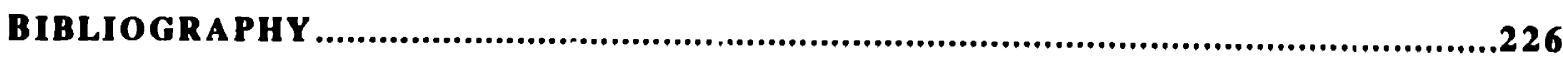




\section{LIST OF FIGURES}

Figure 1.1 - Example Radar Calculation for 3-meter Resolution........................................................17

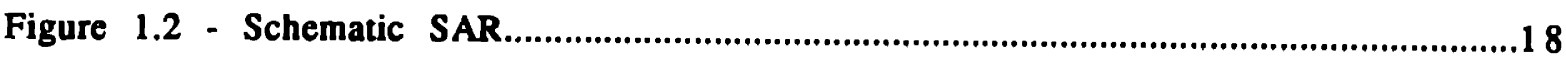

Figure 2.1 - Rectangular Piston in an Infinite Baffle..............................................................38

Figure 2.2 - Top view, rectangular piston in an infinite baffle

with example radiation pattern (beampattem)............................................................40

Figure 2.3 - Rectangular piston main lobe, geometry \& dimensions..................................................41

Figure 2.4 - Side scan sonar. Genmetry and image display............................................................43

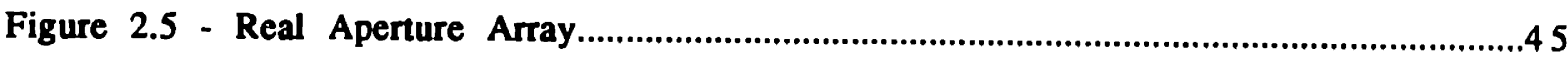

Figure 2.6 - Synthetic Aperture via Geometry.......................................................................48

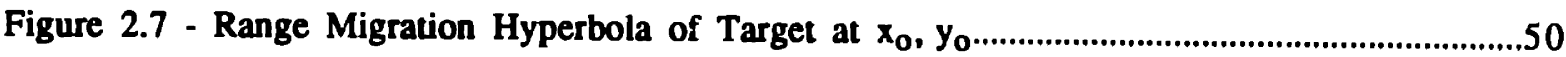

Figure 2.8 - Similar Triangles Argument.............................................................................52

Figure 2.9 - Ensemble Time Series for a Single Target...............................................................53

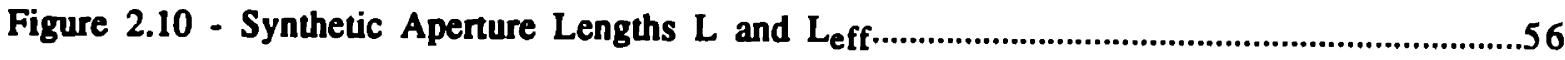

Figure 2.11 - Geometry for Unfocused SAS.........................................................................61

Figure 2.12 - Geometry for Spotlight Synthetic Aperture...........................................................64

Figure 2.13 - Transform Pair: Geometry and Wavenumber Spectra..................................................68

Figure 2.14 - Transform Result of Fig. 2.13 .......................................................................7 1

Figure 2.14' - Transform Pair: Transmit Signal and Frequency Spectra...............................................74

Figure 2.15 - Geometry for Target, Alias Target Image, and Null of Sonar Beampattern.........................76

Figure 2.16 - Ensemble Time Series of Target Echoes Showing Range Migration and Beampattern Weight................................................................................76

Figure 2.17 - Along-Track Correlation at Target Point..................................................................79

Figure 2.18 - Along-Track Correlation at Alias Point..................................................................79

Figure 2.19 - Along-Track Image "Slice" Showing Images of Target and Alias Target............................79

Figure 2.20 - Along-Track Correlation at Target Point for Reduced Aperture.........................................81

Figure 2.21 - Along-Track Correlation at Alias Point for Reduced Aperture..........................................81

Figure 2.22 - Along-Track Image "Slice" Showing Images of Target and Alias Target for Reduced Aperture......................................................................................8 1

Figure 2.23 - Sequential Hydrophone Array to Satisfy Spatial Sampling............................................85

Figure 2.24 - Transform Pair: Geometry with Amplitude Weight and Wavenumber Spectra....................89

Figure 2.25 - Sonar Mainlobe Taper of Synthetic Aperture............................................................90 
Figure 2.26 - Amplitude Taper of Sonar Beam Pattern on Mainlobe and Sidelobes

of Synthetic Aperture...................................................................................992

Figure 2.27 - Transform Pair: Undersampled Case......................................................................95

Figure 2.28 - Undersampled Case Showing Alias Images...............................................................96

Figure 2.29 - Undersampled, Broadband Wavenumber Spectra (beampatterns)........................................97

Figure 2.30 - Kquivalent Mainlobe Area.............................................................................99

Figure 2.31 - Example One-Sided FM Chirp Spectrum..............................................................100

Figure 2.32 - Composite Wavenumber (beampattem) Spectrum for Broadband Undersampled SAS.........102

Figure 2.33 - "Billboard" SAS for 3-D Imaging......................................................................109

Figure 3.1 - Flowchart for Echo Data Creation...................................................................110

Figure 3.2 - Flowchart for Image Synthesis from Echo Data... ...................................................115

Figure 3.3 - Imaging Geometry, 2-D example..................................................................1 18

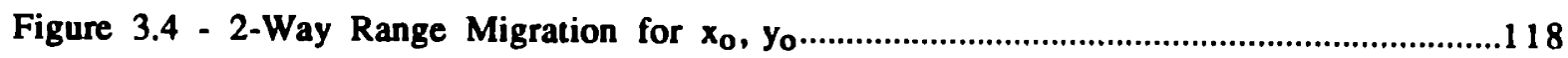

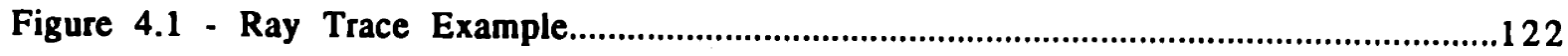

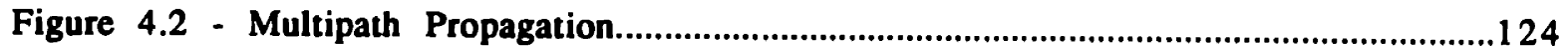

Figure 4.3 - Effect of Sound Speed Profile on Range Migration Arc.............................................128

Figure 5.1 - Geometry for Gough and Hayes Experiment.........................................................140

Figure 5.2 - SAS Geometry for Computer Model..................................................................142

Figure 5.3 - Narrowband Focused Image Showing Target and Alias Targets, Linear Scale......................144

Figure 5.4 - Narrowband Focused Image Showing Target and Alias Targets, Log Scale.........................145

Figure 5.5 - Broadband Focused Image, Linear Scale ..................................................................147

Figure 5.6 - Broadband Focused Image, Along-Track Expanded, Linear Scale.......................................148

Figure 5.7 - Broadband Focused Image, Log Scale...................................................................149

Figure 5.8 - Broadband Focused Image, Along-Track Slice, (66.8 m Slant Range)...............................150

Figure 5.9 - Narrowband Unfocused Image, Linear Scale...........................................................152

Figure 5.10 - Narrowband Unfo'used Image, Log Scale.............................................................153

Figure 5.11 - Broadband Unfocused Image, Linear Scale...............................................................154

Figure 5.12 - Broadband Ủnfocused Image, Log Scale...............................................................155

Figure 5.13 - Multipath Example: Single Target, Smooth Surface, Smooth Bottom............................157

Figure 5.14 - Multipath Example: Single Target, Rough Surface, Rough Bottom................................158

Figure 5.15 - Deep Ocean Image, Matched Profiles...................................................................161

Figure 5.16 - Deep Ocean Image, Strongly Matched Profiles.........................................................162

Figure 5.17 - Deep Ocean Image, Rough Match Profiles............................................................163

Figure 5.18 - Deep Ocean Image, Narrow Sonar Beam....................................................................165

Figure 5.19 - Deep Ocean Image, Narrow Sonar Beam, Mismatch Series...........................................167

Figure 5.20 - Deep Ocean Image, Narrow Sonar Beam, Mismatch Series Continued..............................168 
Figure 5.21 - Deep Ocean Image, Narrow Sonar Beam, Mismatch Series Continued .169

Figure 5.22 - Coherence Time Compared: 120 seconds vs. 14 seconds.

Figure 5.23 - Image Reconstruction Platform Speeds: Matched vs. Mismatch.

Figure 5.24 - Random Platform Motion - Log Scale.

Figure 5.25 - Random Platform Motion - Linear Scale.

Figure 5.26 - Range Taper 179

Figure 5.27 - Azimuth Taper. 180

Figure 5.28 - Azimuthal Images for Target and Alias; $28 \mathrm{kHz}, 10 \mathrm{~Hz}$ bandwidth .183

Figure 5.29 - Azimuthal Images for Target and Alias; $28 \mathrm{kHz}, 750 \mathrm{~Hz}$ bandwidth. 184

Figure 5.30 - Azimuthal Images for Target and Alias; $22.5 \mathrm{kHz}, 15 \mathrm{kHz}$ bandwidth. 185

Figure 5.31 - Unfocused Azimuthal Image Showing Alias Rejection.. .186

Figure 5.32 - Broadband Undersampled Azimuth Images of Target and Smeared Aliases, Full Aperture ( $\mathbf{N}=1)$

Figure 5.33 - Broadband Undersampled Azimuth Images of Target and Smeared Aliases,

Reduced Aperture ( $\mathrm{N}=4)$.

Figure 5.34 - Broadband Undersampled Azimuth Image of Target and Smeared Aliases,

Null-to-Null Aperture.

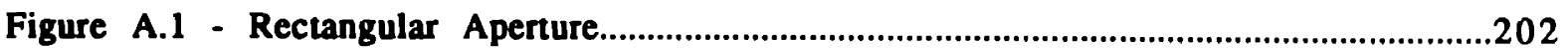

Figure B.1 - One-Dimensional View of Range Resolution........................................................209

Figure B.2 - Transmit, Receive, and Replica Correlation for a Narrowband Signal..............................210

Figure B.3 - Transmit, Receive, and Replica Correlation for a Broadband Signal.................................210

Figure D.1 - 2-D Doppler Geometry...............................................................................217

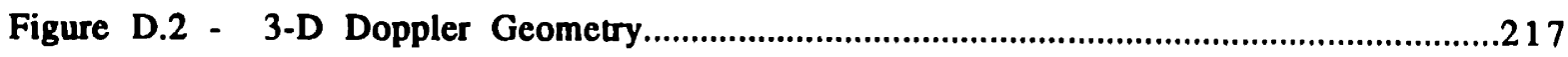

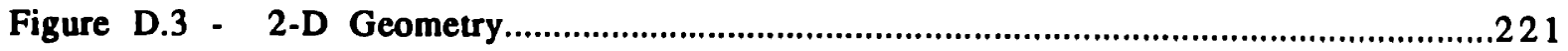

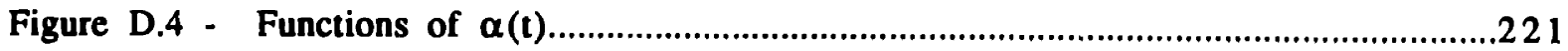

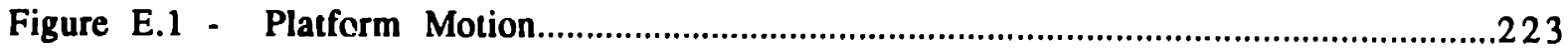




\section{LIST OF TABLES}

Table 1.1 - Synthetic Aperture: Radar versus Sonar..................................................................20

Table 2.1 - Along-Track Resolution Compared...........................................................................66

Table 2.2 - SAR Examples - Aperture Lengths Compared..............................................................83

Table 2.3 - Synthetic Aperture Sidelobe Levels (Natural Weighting) ...............................................93

Table 3.1 - Program Capabilities/Limits...............................................................................1 13

Table 4.1 - Medium Stability Experiment Summary...............................................................131

Table 4.2 - Medium Stability Experiments Compared...................................................................133

Table 5.1 - Operating Parameters for Gough and Hayes SAS Used in the Computer Model..................143

Table 5.2 - Deep Ocean Simulation, $30^{\circ}$ Beam, .10 m/s...........................................................159

Table 5.3 - Deep Ocean Simulation, $5^{\circ}$ Beam, .55 m/s...............................................................164

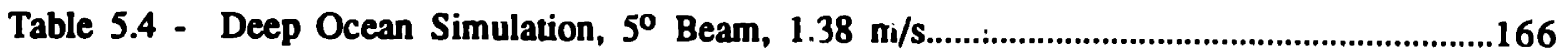

Table 5.5 - Random Platform Motion Examples.....................................................................176

Table 5.6 - Alias Level Relative to Mainlobe........................................................................182

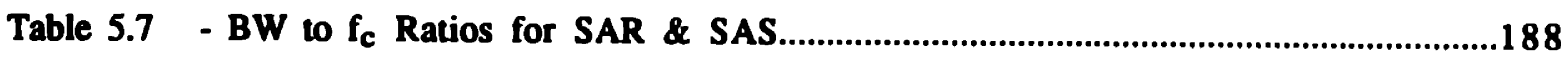

Table 5.8 - Broadband Undersampled Alias Levels Compared: Equation 2.74 vs. Computer Model,

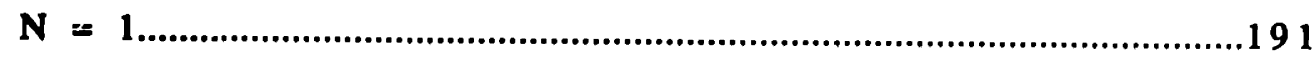

Table 5.9 - Broadband Undersampled Alias Levels Compared: Equation 2.74 vs. Computer Model,

$$
\mathbf{N}=4
$$




\section{NOTATION AND LIST OF SYMBOLS}

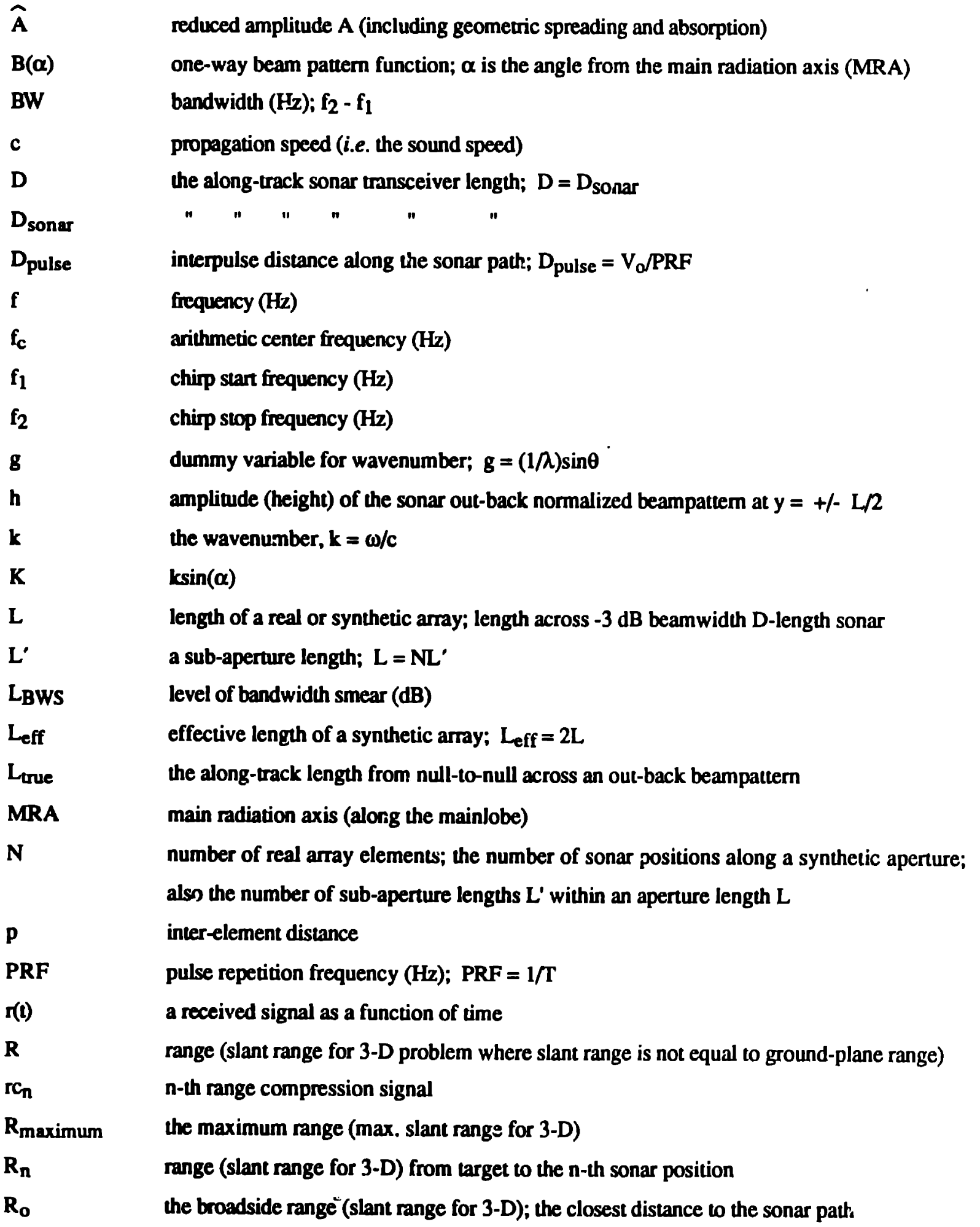




$\begin{array}{ll}s(t) & \text { a transmitted signal as a function of time } \\ S N R & \text { signal to noise ratio } \\ t_{n} & \text { time of flight for an echo from the } n \text {-th sonar position } \\ T & \text { interpulse period (seconds) } \\ T_{\text {cohere }} & \text { the coherence time } \\ T_{\text {elapsed }} & \text { elapsed time (seconds) } \\ u & \text { a dummy variable for wavenumber; See "g" } \\ x & \text { the } x \text {-axis; used for the range axis (or slant range for 3-D) } \\ y & \text { the y-axis; the platform path. } \\ V_{0} & \text { the sonar platform velocity along the } y \text {-axis }\end{array}$

$\boldsymbol{\alpha}$

angle from the main radiation axis to the target for beampatterns; angle from forward along-track direction to the target for Doppler geometry.

$\boldsymbol{\beta}$ beampattern weight (i.e. $\boldsymbol{\beta}^{2}$ is the normalized out-back beampattern weight.)

$\Gamma$ broadband composite mainlobe amplitude for the wavenumber spectrum for a target, relative to an alias lobe smear level of $1.0(\Gamma>1.0)$

$\lambda$ wavelength

$\lambda_{c}$

arithmetic center wavelength

$\lambda_{1}$

chirp start wavelength

$\lambda_{2}$

chirp stop wavelength

$\pi$

3.14159...

$\Delta \theta$

$\theta$

rotation angle for the boresight of a spotlight synthetic aperture transceiver

$\theta_{\text {mainlobe }}$ angle from the MRA

$\theta^{\prime}$

radian $\mathbf{- 3} \mathrm{dB}$ width of the mainlobe

$\Phi$

angle of the alias lobe from the MRA

Palong-track

dimensionless quantity for propagation stability

Prange

along-track resolution; $\rho_{\text {alung-track }}=\rho_{y}$

Py

range direction resolution; cross-track resolution

$\boldsymbol{\sigma}$

$y$-direction resolution

target reflectivity (1.0 maximum)

$\sigma_{\mathrm{rms}}$

variance

$\tau$

pulse time duration

$\boldsymbol{\omega}$

radian frequency 
depression/elevation angle from the horizontal for Doppler geometry dimensionless quantity for propagation stability; normalized by $\left(\sigma_{\mathrm{ref}} / \sigma_{\mathrm{rms}}\right)$ dimensionless quantity for propagation stability; normalized by $\left(\sigma_{\mathrm{ref}} / \sigma_{\mathrm{ms}}\right)^{2}$ 


\section{Chapter 1}

\section{INTRODUCTION}

This chapter provides an overview on the notion of the synthetic aperture, or equivalently the synthetic array. A brief introduction is given of the work in synthetic aperture radar (SAR) and how the synthetic aperture principles carry over from the electromagnetic-wave radar environment to the acousticalwave sonar environment, resulting in synthetic aperture sonar (SAS). The previous work in synthetic aperture sonar is reviewed, and the contributions of this thesis to the field of SAS are summarized. The chapter concludes with the organization of the thesis.

\subsection{History and Overview}

Radar (radio detection and ranging) and sonar (sound navigation and ranging) were both developed as means for detecting objects that could not be observed visually. Radar was originally invented and demonstrated (in Germany circa 1904) by Christian Hülsmeyer to locate and prevent collisions between ships at sea both in darkness and in fog [Pritchard, 1989]. Radar was later developed in several countries, notably Germany, Great Britain, and the United States. Radar did not become truly operational until the mid 1930s, primarily in Germany, and sometime later (by necessity) in Great Britain. The British accurately imagined that they lagged the Germans in the development of radar as an early waming tool and undertook a massive development effort. One of their developments was the first airbome radar, which was intended to help British fighters find enemy planes during night operations, or in cloudy conditions. What was not expected was that the airborne radar accidentally imaged the ground below, and gave radar a mapping and imaging capability not previously envisioned. This was also observed, several years later, at the MIT Radiation Laboratory where a 3-cm (wavelength) high frequency airborne radar was shown to give a fairly accurate and detailed image of Cape Cod [Garmon, 1989]. The Cape Cod image is prominently displayed within a multicavity magnetron as the logo for the Radiation Laboratory Series of books on radar which were published after the war [see MIT Rad. Lab]. So began the use of radar as an imaging tool.

Sonar, meanwhile, had been routinely used for navigation and food-finding purposes by bats and cetaceans (whales) but was not used by man for echolocation until the early 1910s. The first practical uses of sonar were shown by the Canadian Reginald A. Fessenden, of the Submarine Signal Company, Boston, MA during the period from 1912 to 1914 [Fay, c. 1944]. Fessenden's invention was a waterproofed 
reciprocating induction motor designed to radiate sound at $540 \mathrm{~Hz}$, and it was the first active sonar ever constructed. It is usually referred to as the Fessenden oscillator. It was conceived by Fessenden as an instrument for underwater communication from ship to ship, and more importantly from ship to submarine, and used the Morse Code as the carrier. This pioneering use of underwater sound communication was demonstrated in Boston Harbor during January 1914. Later, and somewhat in response to the 1912 sinking of the ocean passenger liner the Titanic after a collision with an iceberg, it was thought that the oscillator could be used as an early waming device to locate icebergs. In March 1914 on the Grand Banks off Newfoundland, Canada, and aboard the U.S. Coast Guard cutter Miami, an iceberg was detected with the oscillator at a range of about 3200 meters, and by accident it was observed that the ocean depth could also be measured. In these landmark experiments, Fessenden showed telemetuy, echolocation and depthsounding. Sonar was thereafter recognized and developed as means for detecting and locating submarines by surface ships, as well as being used by submarines to find their noisier targets. Though not nearly as surprising and dramatic as in radar, sonar was also discovered to have imaging capabilities [Flemming, 1982].

What these early radars and sonars had in common was that they transmitted and received their energy by a combined transmitter-receiver (or transceiver) having an aperture. The aperture is the window through which the energy passes, either for transmission or receiving. Antenna is synonymous with aperture. Apertures are frequently given length and width dimensions per wavelength: e.g. the radar cish (the aperture) has a 0.5 -meter diameter, and the wavelength for $1 \mathrm{GHz}$ (the transmit frequency) is $0.3 \mathrm{~m}$, so the dish diameter is about 1.6 wavelengths. This is calculated from the following relation:

$$
\lambda=\frac{\mathbf{c}}{\mathbf{f}}
$$

$$
\text { where } \begin{aligned}
c & =3 \times 10^{8} \mathrm{~m} / \mathrm{s} \text { (i.e. the speed of light) } \\
\mathrm{f} & =1 \mathrm{GHz} .
\end{aligned}
$$

Therefore, $\lambda=0.3$ meters (i.e. the wavelength) and

$$
\frac{\text { radar dish diameter }}{\text { distance per wavelength }}=\frac{0.5 \mathrm{~m}}{0.3 \mathrm{~m} / \lambda}=1.6 \lambda
$$

The number of wavelengths across an aperture is inversely related to the beamwidth. A large number of wavelengths across an aperture, say greater than 10 , would give a fairly narrow transmit beam (for an active system), and likewise give a narrow receive beam (in a passive system). Note that the term "active" implies the combined use of transmitting a pulse and listening for the echo, and it is a term which applies to both radar and sonar. "Passive" implies the listen-only operation of a radar or sniliar, and relies on the 


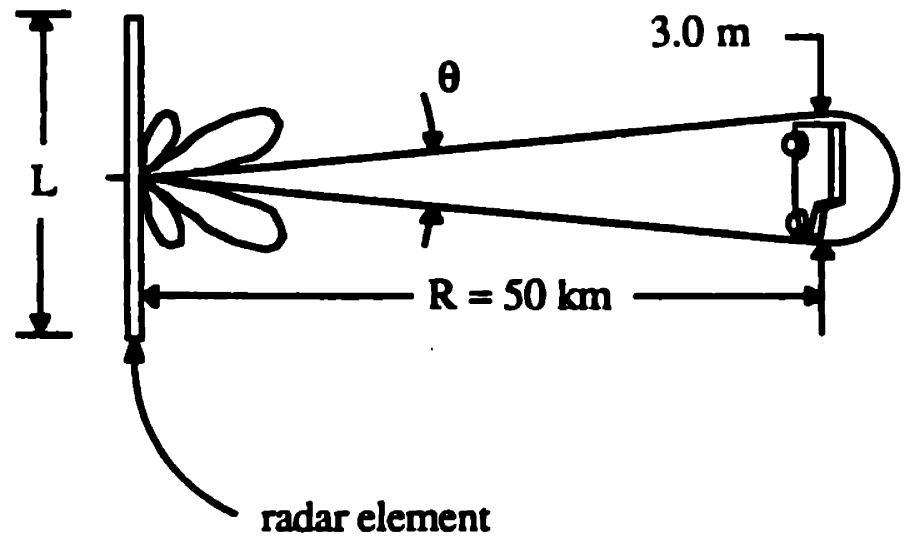

$$
\begin{array}{ll}
\mathrm{f}=1 \mathrm{GHz} & \\
\lambda=0.3 \mathrm{~m} & \theta=\lambda / \mathrm{L} \\
\mathrm{R}=50 \mathrm{~km} & \text { resolution } \rho=\theta \mathrm{R} \\
\mathrm{L}=? &
\end{array}
$$

$$
\begin{aligned}
3.0 & =\theta R \\
& =(\lambda / L) R
\end{aligned}
$$

therefore $\mathrm{L}=5000 \mathrm{~m}$

Figure 1.1 - Example Radar Calculation

for 3-meter Resolution 


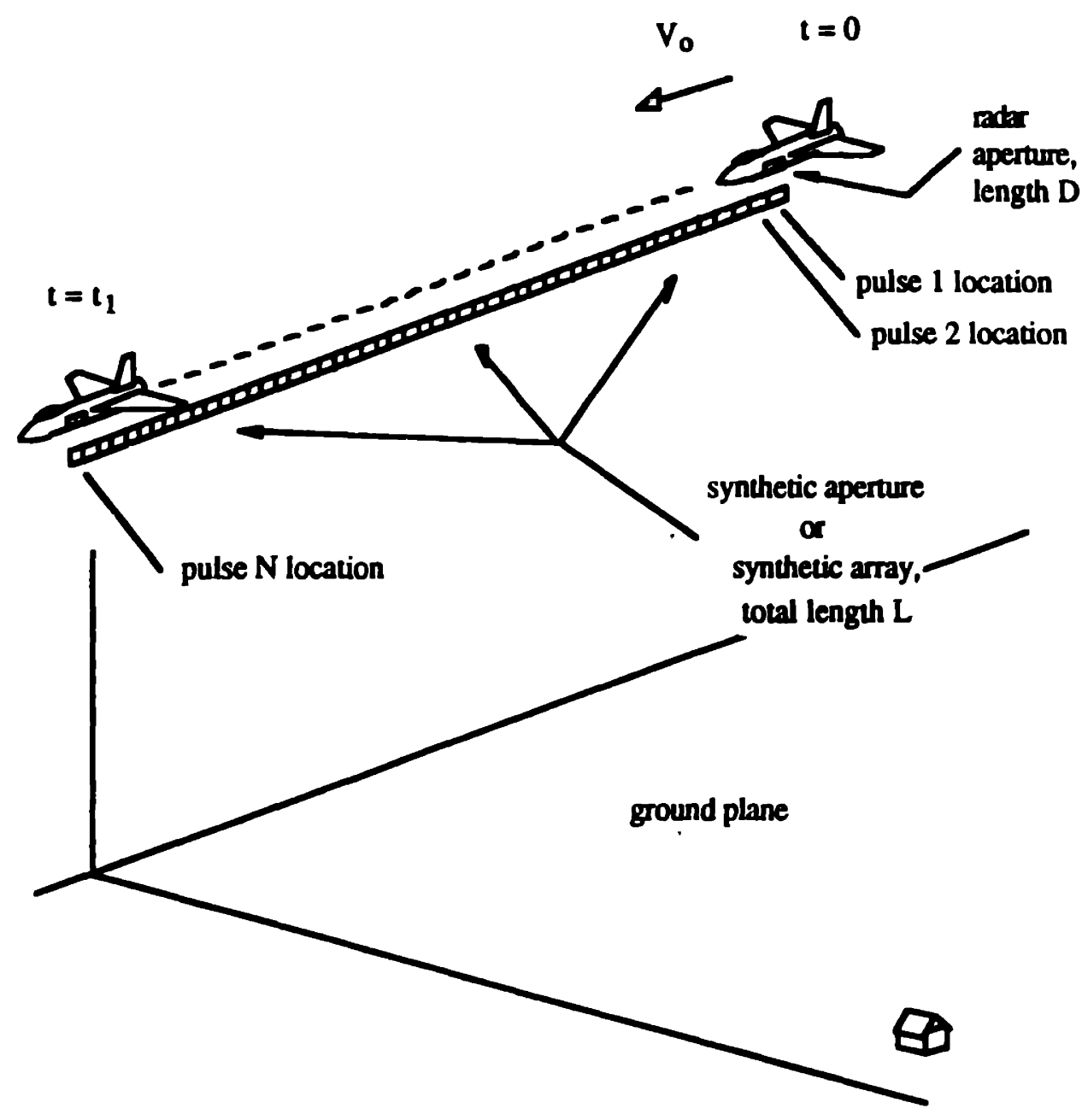

Figure 1.2 - Schematic SAR 
presencs of a noise-making target. These features apply to apertures for many types of wave propagation in acoustics (specifically sonar), in electromagnetics (specifically radar), and in nptics. A further description of the relation between wavelengths to aperture size, and the resulting beamwidh and directivity for apertures which transmit or receive energy, is given in Appendix A.

\subsubsection{Synthetic Aperture Radar (SAR)}

The smaliest object that may be resolved by a radar or by a sonar determines its imaging ability, and this is directly related to the wavelength and aperture size of the radar or sonar. Radars or sonars that have apertures that are many wavelengths across the length, width, or both length and width of the aperture will have good imaging capability. As an example, if a radar is to be designed to image a 3.0-meter sized target (the size of an automobile) at a range of 50 kilometers $(\mathrm{km}$ ) and at a frequency of $1.0 \mathrm{GHz}$, the radar aperture must be at least $5000 \mathrm{~m}$ long as shown in Figh ': 1.1. A radar of this size would be too large and too expensive, and perhaps too heavy for either an aircraft or spacecraft. However, a radar having much smaller dimensions (i.e. a smaller aperture, like 10 meters) could be progressively moved along a straightline path, and perform successive transmit and receive operations. If the successive receptions (or echoes) were suitably processed, an equivalent large-sized aperture could be formed that equaled the performance of the $5000 \mathrm{~m}$ antenna. This is shown schematically in Figure 1.2. This successive radar transmit and receive operation along a known path (in this case, a straight line) and subsequent signal processing is known as an aperture synthesis technique, where a radar aperture is synihesized from the successive use of a small, real aperture radar, --- this is called synthetic aperture radar (SAR). Synthetic aperture radar is also called synthetic array radar.

So a large aperture (or array) may be synthesized by using a small aperture transducer (in this example, a radar) and by processing the signals appropriately, accounting for the change in geometry as the small aperture transits along a path. This concept of replacing a large, impractical antenna with the combination of a much smaller antenna and signal processing is attributed to Carl Wiley in 1951. During the 1950s, several groups investigated this idea, notably the group at the University of Michigan on Project Wolverine. Since then, SAR has been extensively studied and developed, and is used as a research, sensing and radar mapping tool. Airborne and spaceborne SAR images are often so good that they rival, and give the mistaken appearance of, optical photographs. There is a vast literature on the subject of SAR, which

will not be addressed in this thesis. However, a better introduction to the history, development, theory and uses of SAR may be found in the books by Kovaly [1980], Hovanessian [1980], Fitch [1988], and Elachi [1988]. The reader is also directed to the tutorial review of SAR by Tomiyasu [1978]. 


\section{Table 1.1 - Synthetic Aperture: Radar versus Sonar}

\begin{tabular}{|c|c|c|c|c|}
\hline & \multirow[b]{2}{*}{ Radar } & \multicolumn{3}{|c|}{ active sonar } \\
\hline & & ocean-based & biomedical & NDT \\
\hline environment & $\begin{array}{c}\text { atmosphere (air) } \\
\text { or } \\
\text { spaceborne }\end{array}$ & ocean & tissue & solids \\
\hline platforms & aircraft or spacecraft & $\begin{array}{l}\text { sea surface or } \\
\text { sub surface }\end{array}$ & guided & guided \\
\hline platform speed & $\begin{array}{c}200 \mathrm{~km} / \mathrm{hr} \text { (aircraft) } \\
\text { to } \\
27,000 \mathrm{~km} / \mathrm{hr} \text { (spacecraft) }\end{array}$ & $\begin{array}{l}<37 \mathrm{~km} / \mathrm{hr} \\
(<20 \mathrm{kn})\end{array}$ & $?$ & $?$ \\
\hline $\begin{array}{l}\text { propagation speed } \\
(\mathrm{m} / \mathrm{s})\end{array}$ & $3 \times 10^{8}$ constant & $\approx 1500$ var. a & $\approx 1500$ var. $^{b}$ & $1500-6000$ \\
\hline waves & $\begin{array}{l}\text { transverse radio: } \\
\text { horiz., vert., circular } \\
\text { polarization }\end{array}$ & $\begin{array}{l}\text { acoustic } \\
\text { (longitudinal) }\end{array}$ & acoustic & $\begin{array}{l}\text { acoustic } \\
\text { vertical shear } \\
\text { hor. shear. }\end{array}$ \\
\hline frequencies & $\begin{array}{l}.45 \mathrm{GHz} \text { (P-band) } \\
\text { to } 10 \mathrm{GHz}(\mathrm{X} \text {-band) }\end{array}$ & $\begin{array}{l}5 \mathrm{kHz} \text { to } \\
650 \mathrm{kHz}\end{array}$ & $\begin{array}{l}1 \mathrm{MHz} \text { to } \\
5 \mathrm{MHz}\end{array}$ & $\begin{array}{l}1 \mathrm{MHz} \text { to } \\
50 \mathrm{MHz}\end{array}$ \\
\hline wavelength & $\begin{array}{l}67 \mathrm{~cm} \\
\text { to } 3 \mathrm{~cm}\end{array}$ & $\begin{array}{l}.3 \mathrm{~m} \text { to } \\
2.3 \mathrm{~mm}\end{array}$ & $\begin{array}{l}1.5 \mathrm{~mm} \text { to } \\
.3 \mathrm{~mm}\end{array}$ & $\begin{array}{l}3 \mathrm{~mm} \text { to } \\
.075 \mathrm{~mm}\end{array}$ \\
\hline geographic references & $\begin{array}{l}\text { many } \\
\text { GPS } \\
\text { groundbased tracking }\end{array}$ & few reliable & not needed & not needed \\
\hline target ranges & $1 \mathrm{~km}$ - to interplanetary & $10 \mathrm{~m}$ to $10 \mathrm{~km}$ & $<15 \mathrm{~cm}$ & $<15 \mathrm{~cm}$ \\
\hline applications & $\begin{array}{l}\text { topographic mapping, } \\
\text { surveillance and } \\
\text { many others }\end{array}$ & $\begin{array}{l}\text { hydrographic } \\
\text { mapping, } \\
\text { object finding. }\end{array}$ & diagnosis & flaw detection \\
\hline
\end{tabular}

a. but varies with depth, temperature, salinity, time and location.

b. varies within a few percent from one tissue type to another. 


\subsubsection{Synthetic Aperture Sonar (SAS)}

The development of synthetic aperture (or equivalently synthetic array) radar has progressed considerably since the 1950 s and is still under intense development today. The superb near-photographic images resulting from SAR led to the idea of using the synthetic aperture method in sonar; however the idea was not explored in the (unclassified) literature until the mid- to late-1960s. Essentially the basic principle of synthesizing a large aperture from the combined use of signal processing and sequential placement of small, real apertures is the same for the radar ard sonar cases. The specific details of the processing will be shown in a later chapter.

The most important differences between the radar and sonar synthetic apertures have to do with (1) the environment, and (2) the platform. Table 1.1 illustrates some of these differences and gives examples.

In SAR the wave travels in essentially straight lines at the speed of light, and is relatively insensitive to changes in space or in the atmosphere. In SAS however, the acoustic wave travels at a speed of about five orders of magnitude less that the radar wave travels in air, or in space. Furthermore, the acoustic wave is readily influenced by temporal and spatial changes in the ocean, and is affected by refraction due to a depth-dependent sound speed profile. Another problem influencing SAS more often than SAR is that acoustic waves can find their way from the platform to a target through several paths, or multipaths. SAS must contend with the potential for multipath transmission and reception from not only the water medium (often referred to as the 'water column') but also from reflections and 'bounces' irom the ocean surface and bottom.

The high propagation speed of a radar wave, coupled with high platform speed, gives the SAR an advantage in imaging distant objects in a short integration time, or dwell time (the time needed to traverse the synthetic array length), which are on the order of a second [Tomiyasu, 1978]. In SAS both the wave and the platform travel at much lower speeds, and so a much longer integration time is needed to travel the length of the aperture (from a minute to ten minutes, e.g.). The longer integration time of a SAS makes the platform more sensitive to platform motion. Because synthetic apertures (synthetic arrays) rely on precise knowledge of phase, it is extremely important to have a stable platform from which to operate. If the platform moves due to external influences such as turbulence or an ocean curreit, then sensors must be available to correctly measure the platform motion, so that the synthetic aperture data may be appropriately compensated, and the motion may be 'removed' from the measurement. The high speril .nul short integration times available to the airbome or spacebome SAR contrast the low speed, lonc in' ' gration time 
of the SAS, so the SAS platform is arguably more sensitive to influence from the environment than the SAR platform.

\subsection{Previous Work in Synthetic Aperture Sonar (SAS)}

The previous work in synthetic aperture sonar (SAS) is reviewed here. At the outset of this research in the Fall of 1988, it was generally thought that few studies and fewer experiments had been performed on SAS. Despite this initial premise, a number of papers have been located in the subject of synthetic aperture sonar (SAS) literature, but only a few unclassified experimental systems are known to have been constructed and successfully demonstrated. Preston Jr. and Kreuzer [1967] used a synthetic holographic $5 \mathrm{MHz}$ ultrasound system to image the interior and exterior of optically opaque objects, and compared their results of an ultrasonic hologram of a machine bolt with an optical photograph. The system used to record the raw data and to froduce the holographic image was similar to the optical processor system often used in SAR processing. In late 1967, the first of three extensive reports [Walsh, 1967; Walsh, 1968; and Chramiec and Walsh, 1971], a patent [Walsh, 1969], and a paper [Walsh and Moss, 1970] on synthetic aperture processing and medium stability as applied to sonar were issued by the Submarine Signal Division of the Raytheon Company, as part of a feasibility sudy for high resolution ocean bottom mapping. This study centered on a surface-ship mounted, $5 \mathrm{kHz}$ synthetic aperture sonar whose purpose was to map the ocean bottom, and in particular, identify objects such as bottom-moored explosive mines . It is somewhat coincident that these two pioneering works nearly cover the entire frequency operating range of the entire work on SAS since 1967 (from $5 \mathrm{kHz}$ in the Raytheon studies to $5 \mathrm{MHz}$ in the experiment of Preston Jr. and Kreuzer.)

In parallel with these two works, was the patent of Flaherty et alia [1970] in which many of the

processing features common to contemporary SAR systems were covered including optical processing and electronic signal processing. The Flaherty et alia patent specifically refers to ultrasonic frequencies while the Walsh patent is more general and does not specify a frequency. The intent of each design is obvious from their respective illustrations and references: Flaherty et alia is intended for medical ultrasonics, mentions a typical operating frequency of $5 \mathrm{MHz}$ and references the work of Preston Jr. and Kreuzer; Walsh's patent is clearly intended for an ocean acoustic design. 
The SAS literature has expanded since the late 1960s, although not to the same extent as SAR literature. Historically, synthetic aperture sonars in the frequency range above $1 \mathrm{MHz}$ were usually intended for biomedical ultrasonics, or for nondestructive testing (NDT) and inspection; SAS systems used below 1-MHz are mostly for use in underwater, ocean acoustics. This provides a natural division for reviewing the SAS literature, and so it is divided into three sections: (1) 'low' frequency active SAS, where 'low' is arbitrarily defined as less than $1 \mathrm{MHz}$; (2) 'high' frequency active SAS (i.e. atove $1 \mathrm{MHz}$ ); and (3) passive SAS. Though the emphasis in this thesis is on applications of 'low frequency' SAS for ocean acoustic imagery, the work on passive synthetic apertures is also included. The ability to create a passive synthetic array demonstrates useful medium stability, so results of passive synthetic array experiments are useful in a study of active SAS, because they both rely on medium stability. The review of the previous work in the closely-related topic of medium stability will be postponed until a later chapter where it becomes more relevant.

A further note to a reader who is unfamiliar with the nomenclature: active SAS is analogus to SAR; in both instances, a signal is actively transmiued and echoes are received. Both the active SAS and the SAR process the echoes into an image (of sorts) of the target (the sea floor e.g. for SAS, and geographic terrain for the SAR).

Passive SAS, on the other hand, does not form an image of an object; instead the passive SAS attempts to identify noise sources and determine their relative bcaring. A practical example of passive SAS could have a ship-towed hydrophone array (a real aperture) which is used to create synthetic apertures in order to locate an enemy submarine more accurately than by using only the real aperture hydrophone array. At present there is no direct radar analog to the passive synthetic aperture sonar. 


\subsection{1 'Low' Frequency Active SAS (below $1 \mathrm{MHz}$ )}

Brown [1968] discussed the application of synthetic aperture methods for high resolution sonar in a classified paper for the Mine Advisory Committee of the (U.S.) National Research Council.

Castella [1971] cast the synthetic aperture in terms of a one-dimensional holographic technique for a mapping sonar system. A signal-to-noise ratio analysis showed 0.1 meter resolutions were possible out to a slant range greater than 1 kilometer for transmission frequencies in the 5- to $30-\mathrm{kHz}$ band. Phase errors demanded correction to better than $40^{\circ} \mathrm{ms}$ for good performance, and 3-dimensional imaging is possible if a vertical array (perpendicular to both platform path and broadside) were included in the system. (The use of a vertical array suggested by Castella to achieve 3-D imaging is an idea that has been overlooked in every subsequent SAS paper which follows in this review of the literature.)

Bucknam et alia [1974] referred to, and extended the principles given in the Raytheon reports by introducing a "coherent, fixed-frequency technique" which was in contrast to Raytheon's frequency diversity method. Also in 1974, Loggins et alia reported on a prototype SAS constructed at the U.S. Naval Coastal Systems Center (NCSC) and the results were published in a classified journal. (See also Stowe [1974] and Stowe [1975] for related work.)

Nitadori [1975] presented a multi-beam scanning method of imaging using fixed arrays of separate transmitters and receivers, and suggested that the use was closely related to aperture synthesis. The experimental system designed from this approach was shown by Nitadori et alia [1980], and used a $4 \times 4$ transmitter array at $200 \mathrm{kHz}$, and a $32 \times 32$ receiver array. The system power was about 200 watts, it had a 2- to 100 -meter range, and the experimental beamwidth was $0.40^{\circ}$ as compared to the theoretical $0.33^{\circ}$.

Cutrona, an investigator and author on synthetic aperture radar in the 1950s and 1960s, published two papers on synthetic aperture sonar [1975 and 1977] which compared the performance of synthetic-aperture techniques with the performance of nonsynthetic-aperture systems. Hanish [1975] reviewed SAS for the U.S. Naval Sea Systems Command (NAVSEA), and concluded that "the random fluctuations in the acoustic properties of the ocean made SAS a difficult, but not an impossible, sonar tool". Hughes [1977] discussed the synthetic aperture method and advantages/disadvantages, and compared a side looking, $200 \mathrm{kHz}$ search sonar with a $3 \mathrm{kHz}$ SAS system; the SAS system was shown to have 150-times better mapping rate and 18-times the range (for identical azimuth and range resolutions) than for the side looking system. Lee [1978 and 1979] showed the synthetic aperture theory and suggested several ways to improve the mapping 
rate while maintaining resolution. Gilmour [1978] patented a "Synthetic Aperture Side-Looking Sonar System" which included Flahery et alia [1970] as a cited reference but did not include Walsh [1969].

McKinney [1982] presented an overview of high-resolution sonar developments and compared various techniques to give high along-track resolution including large real apertures, synthetic apertures, and interferometry arrangements.

In 1982, Loggins et alia, in an unclassified publication of NCSC work, presented results from a specially designed SAS rail facility to collect data from 20 to $100 \mathrm{kHz}$. The rail guide was used to eliminate unwanted, uncontrolled platform motion. A number of known targets were seeded on the bottom to evaluate the beamforming performance of the SAS system. It was found that the azimuth sidelobes had levels of about $-17 \mathrm{~dB}$. In a separate paper, Loggins [1982] discussed the differences between the radar and sonar implementations of the synthetic aperture method, and showed a performance comparison between SAS and a conventional sonar. A vernier technique to increase the mapping rate, and incoherent averaging to reduce image speckle were also shown.

Pusone and Lloyd [1984] showed results from a computer model which calculated the synthetic beam pattem of a multi-ping (multi pulse) synthetic aperture sonar incident on a fixed scatterer. The model used a 3-element hydrophone array which enabled real aperture beam steering, and thereby emulated spotlight SAS. The results show the degradation due to deviations from a straight-line course and due to a low signal-tonoise ratio. The results were in the form of beam patterns focused at fixed ranges of $750 \mathrm{~m}$ and $15 \mathrm{~km}$.

de Heering [1984] reviewed the basic principles of SAS and then suggested several altemate operating and processing schemes including coherent averaging (a modified unfocused SAS method), broad-band transmission to increase platform speed while avoiding grating lobes (and consequent alias images), and envelope processing. Several years later de Heering reported these features as well as others in his Ph.D. thesis [1989].

Gough [1986] discussed a new approach to synthetic aperture sonar using continuous transmission frequency modulation (CTFM). Gough also introduced a phase-differential method which may also be used to form a synthetic aperture image. To further investigate the combined use of CTFM and SAS, an airacoustic experiment was conducted and was scaled appropriately in frequency and length, thus making a useful comparison to the underwater environment. The target images generated were shown as contour plots. 
Brekhovskikh et alia [1987] discussed an experiment using a conventional $12 \mathrm{kHz}$ transmitter array and two receiver arrays ( $2.4 \times 2.4 \mathrm{~m}, 64$ hydrophones; and $2.7 \times 2.7 \mathrm{~m}, 256$ hydrophones) to image the 4000 meter deep ocean bottom. The transmitter/receiver systems were used to image the bottom using the real aperture and using a synthetic aperture of the real array formed by the drifting ship.

In 1987, Dutkiewicz and Denbigh reported a three-part experiment in which they were concerned with imaging water-bome and sub-bottom targets. In the first part, two mid-water ping-pong ball targets were imaged in a test tank using an 8-mm square transducer operating at $200 \mathrm{kHz}$, and formed a $385 \mathrm{~mm}$ aperture from 78 sampling points. Both focused and unfocused images were successfully formed, and reasonable focused images were formed when random phase errors, and when a $10 \%$ platform velocity error were individually and intentionally introduced. The second experiment consisted of testing a $4.5 \mathrm{kHz}$ (approximately a 100-degree real aperture beamwidth) sub-bottom penetrating system from a floating laboratory on a fresh water reservoir. The transducer was suspended from a rail-based trolley and was capable of apertures up to 4.5-meters-long. Though no attempt was made to place known test targets on the bottom (as in the 1982 work of Loggins et alia) and in the sub-bottom for experiment validation, images resulting from both the $4.5 \mathrm{kHz}$ SAS system and a $300 \mathrm{kHz}$ side scan system showed reasonable agreement. The third experiment was an at-sea test of the same $4.5 \mathrm{kHz}$ system as mounted rigidly between the hulls of a two-engine catamaran. The apertures formed were 85-meters-long. A number of problems occurred with this test: the platform speed was not accurately known (and had to later be estimated by trial and error), there was no evidence of motion compensation, and strong acoustical interference was noted which the authors attributed to multiple reflections from the boat hulls. In spite of these difficulties, strong similarity in images of the same area of the sea bed was found when processing sets of data having different squint angles (pointing the sonar transmitter/receiver slightly away from broadside, either in the forward or backward direction). This indicated to the authors that some confidence could be had in the images.

Tarng and Yang [1987] showed the results of a numerical simulation of a $3 \mathrm{kHz}$ synthetic aperture sonar inaging a target located 1000 meters deeper that the sonar platform. The propagation model used raytracing and assumed a simplified isogradient sound speed profile. The results showed the relation of alongtrack resolution, platform speed, eigenray angle, and the pulse repetition frequency (PRF).

Shishido [1988] cited the trembling motion of the sonar platform as the reason for the lack of practical use of the synthetic aperture sonar. The similarity of SAS with common depth point (CDP) stacking seismic exploration was also made. The effects of platform motion were then shown by computer simulation to determine an acceptable range for the amplitude and period of uncompensated motion. 
Gough and Hayes [1989] tested a prototype wire-guided SAS in Loch Linnhe, Scotland. Their system used continuous transmission frequency modulation (CTFM) in the 15- to $30-\mathrm{kHz}$ band. An air-filled, 1.2$\mathrm{m}$ diameter steel sphere was used as a test target and was placed at about 66-m broadside from the taut wireguideway and at a depth of about 8-meters. The wire-guide prevented unwanted lateral platform motion and the platform was towed at $1 \mathrm{kn}(.517 \mathrm{~m} / \mathrm{s})$. The image results confirmed that alias target images are formed for narrowband operation, when the platform travels too fast and spatial undersampling occurs. Aliasing can be eliminated by using a slower platform speed (to satisfy spatial sampling) but this comes at the expense of the mapping rate. A high platform speed may be used (and the mapping rate preserved) by using wideband frequency operation. The high operating speed violates the spatial sampling (in the along-track direction) which would ordinarily introduces alias target images (the same as alias lobes in a beam pattern). However, the images resulting from wideband operation eliminated (smeared) the aliasing lobes so that only the single target was found. The wideband images also appeared to agree with the theory for theoretical resolution of synthetic apertures in both azimuth (in the platform-tow direction, or along-track) and range (or cross-track) directions. These results were also reported in the Ph.D. thesis of one of the authors [Hayes, 1989].

More recent is the NCSC $650 \mathrm{kHz}$ based system [NCSC, 1989], the highest frequency SAS system found in the literature for ocean acoustic purposes and was intended for short range imagery. Imaging under the influence of ocean and platform effects was shown by Rolt et alia [1990] where their computer-based imaging model was compared and agreed with the experimental image results of Gough and Hayes [1989].

Two special examples of synthetic aperture sonars used to image the ocean bottom or to determine its properties are the North Allantic Transect by the NAT Study Group [1985], and the synthetic aperture array technique of Werby et alia [1987]. The Norh Atlantic Transect (NAT) experiment was a wide-aperture twoship multichannel seismic study of the oceanic crust. Each ship had a stern-deployed towed hydrophone array (3000- and 3310-m lengths), and each had an air gun system for active transmission. The ships fired their air guns altemately (in an interleaved manner), so that an air gun was fired every 30 seconds, and each ship fired its own air gun every 60 seconds. Both ships measured hydrophone data for all air gun shots. The vessels moved at a nominal speed of $9 \mathrm{~km} / \mathrm{hr}$, and in total more than $3800 \mathrm{~km}$ of common depth point (CDP) data was collected, using a synthetic aperture of more than $10 \mathrm{~km}$ length. The resulting images from the data showed two-dimensional (ship track versus depth) high resolution crustal structure which could not have been attained with a smaller real aperture. The synthetic aperture array technique by Werby et alia, used the self-radiated noise from a ship to insonify the bottom. Meanwhile, an array is towed from the ship to measure the field backscattered from the bottom. By measuring and processing the bottominteracting field, the type and properties of the local sea floor may be inferred. 


\subsection{2 'High' Frequency Active SAS (above 1 MHz)}

Kreuzer [1971] described the basic coherent imaging equations for the propagation of coherent images between two reference planes with and without an aperture lens. This approach is more general than other synthetic aperture approaches found in the literature because the sequential sparsely sampled illumination of an object (e.g. transmit a pulse) may occur in one plane, while the sequential returns (the echoes) are sparsely recorded in a distinct second plane. These two planes, or apertures, can be coincident and the sequential illumination and recording may be done simultaneously; this is the usual approach taken in most studies and experiments conceming SAS. To demonstrate the principles, a clever optical experiment was conducted using photographic transparencies of two different objects.

Sato et alia [1973] described the principles of SAS and fundamental results of a $1 \mathrm{MHz}$ experiment to image the cross-section of a $4 \mathrm{~mm}$ steel wire. The results showed a half-amplitude wire diameter of 4.7 $\mathrm{mm}$ in azimuth, but a $13.2 \mathrm{~mm}$ diameter in range. The poor range result was a consequence of having, at best, a $9 \mathrm{~mm}$ theoretical range resolution.

Burckhardt [1974] et alia described a 2 MHz SAS system that used an optical processor for image reconstruction. The $2 \mathrm{MHz}$ frequency was chosen because it is a typical frequency used for ultrasonic breast examination. The useful range was from $20-$ to $40-\mathrm{cm}$, the lateral scan width was $30 \mathrm{~cm}$, and the resolution in both range and azimuth was $1.5 \mathrm{~mm}$. The experiment was performed in a rubber-lined water tank. A number of different targess were used to test the system including synthetic rubber balls, metal pins, and copper wire grids. The resulting images correctly located the targets. It was thus shown that the experimental 2 MHz SAS system demonstrated a significantly higher lateral (azimuth or along-track) resolution than could be attained with a conventional B-scan system (essentially a side scan sonar) working at the same frequency. The authors did not however, test the system in vivo in this experiment, and used only non-living targets.

Keating et alia [1975] compared the imaging from a $10 \times 10$ hologram (receive) array and a $2 \times 2$ transmitter array with images due to a $10 \times 10$ element array and a single transmitter, and a $20 \times 20$ array and a single transmitter. The experiment was conducted underwater upon two small glass-shell spheres (3$\mathrm{cm}$ diameter), at a separation distance of $4 \mathrm{~cm}$. The image results showed that aperture synthesis may obtain high resolution images with cost savings over conventional arrays.

Dick et alia [1977] summarized synthetic aperture processing in the usial fashion along a hyperlulic geometric range-migration path. They then proceeded to offer alternate geometric paths in an effort li , "isc 
the processing and they showed the point-spread function for these alternate paths. These efforts were part of the design of an ultrasonic imaging system for medical applications.

Sato and Ikeda [1977a] presented a prototype SAS which displays reconstructed images on a CRT screen. The system used a circular scanning transducer operating at $1 \mathrm{MHz}$, and showed experimental results from various scanning depths, showed the effects of a threshold bias, and showed the effects of zooming. The targets were a number of $3 \mathrm{~mm}$ steel balls, and $2 \mathrm{~mm}$ thick aluminum plates.

Sato and Ikeda [1977b] showed the importance of changing the carrier frequency for sequential pulses along an aperture length. This is a sequential variation of wideband operation.

In a separate 1977 paper, Sato et alia [1977c] described two effective preprocessing steps for synthetic aperture sonar. One step is a preprocessing to eliminate the effects of phase turbulence in the propagation medium. The other step uses a broad band transmission to improve range resolution. Two experiments were performed using an existing SAS system, and the results support the authors' ideas.

Ikeda, Sato and Suzuki [1979] extended the principles of Ikeda and Sato [1977b], and showed computer simulations of along-track (azimuthal) images of several point targets.

Ikeda, Sato and Ohshima [1979] described and showed computer simulations of synthetic aperture sonar along-track images that were both perturbed by a turbulent media, and corrected by the method shown in the paper. Ikeda and Sato [1980] further examined the problem of a turbulent medium and showed the results of a MHz-based system. Sato, Ikeda and Endo [1981] showed experimental results of the method described in Sato and Reda [1977b].

Bennett $e t$ alia [1982], and Peterson, Bennet and Kino [1982] presented the results for a 32-element real-time SAS imaging system operating at 3.3 $\mathrm{MHz}$. The performance of the system was demonstrated on both simulated and real flaws, and the system was designed to inspect for flaws in solids samples with longitudinal, shear and surface waves.

Liang et alia [1982] showed a $50 \mathrm{MHz}$ synthetic focus SAS imaging system suitable for reconstructing three-dimensional images of flaws inside silicon nitride. Square synthetic apertures were used to image flaws in flat discs and cylindrical synthetic apertures were used to image cylindrical rod cases.

Thomson [1984] reviewed the synthetic aperture focusing technique (SAFT) and showed expcrimental results for a $4 \mathrm{~mm}$ diameter, $5 \mathrm{MHz}$ system. 
Ikeda et alia [1985] described an image reconstruction algorithm for "disturbed" synthetic aperture sonar data, using an aperture division method.

Moshfeghi [1986] described a synthetic aperture method for ultrasonic NDT operating at $5 \mathrm{MHz}$ which uses the fact that in an NDT application, there are very fcw defects which give rise to echoes. This is exploited by rectifying the few echoes that exist, and convolving the results with a simple filter. The intent is to considerably reduce and simplify the processing, promoting real-time operation, while simultaneously improving the quality of flaw imaging. The author acknowledges that these methods would not work in medical imaging, "where the image field is cluttered and echoes are overlapping."

Kino [1987], amidst his review of various forms of lenseless acoustic imaging, described several systems and operating principles of synthetic aperture imaging (page 262-275). Fitch [1988], in his book on 'JAR, described the differences between radar and acoustic propagation particularly as applied to synthetic apertures. He then described the synthetic aperture focusing technique for ultrasonic testing (SAFT-UT).

Ikeda et alia [1988] described the formation of a B-scan medical ultrasound image by using groups of transducer elements to create a partial aperture, and these partial apertures are then used to create full synthetic apertures. This is a method used in SAR to reduce speckle.

Badalyan and Bazulin [1989] described and showed experimental results of a $2.5 \mathrm{MHz}$ center-frequency scanning system using pseudorandom signals for digital acoustic holography. The azimuth resolution appeared to be equal to that attainable with other wideband systems and was about 1 millimeter.

\subsubsection{Passive SAS}

Williams [1976] presented results from a synthetic aperture experiment using a ship-towed source and midwater receiving hydrophones which were buoyed at depths of 2804- and 3810-meters (9200- and 12,500feet) from a 5242 meter bottom (17,200 feet). Straight-line tows were conducted perpendicular to the hydrophones at ranges of 107-, 172-, 237-, 366-, and 495-kilometers, during which a continuous wave (cw) $400 \mathrm{~Hz}$ signal and a swept frequency modulation signal (350 to $450 \mathrm{~Hz}$ ) were transmitted continuously during the tow. Despite difficulties in maintaining straight tows due to surface swells (waves), a number of passive synthetic apertures were constructed for the $400 \mathrm{~Hz}$ transmission on an intermittent basis. The synthesized lengths were up to 925 meters and corresponded to coherence time intervals of up to 7.5 minutes (450 seconds). 
Carey and Yen [1984] showed results of passive synthetic apertures which were formed with experimental data, from the coherent summation of 1 to 4 hydrophone groups at successive time samples. A calibrated moored source was at $37-\lambda$ depth in $3200 \mathrm{~m}$ of water, and at a distance-to-wavelength ratio of 20,000 to 30,000 from the receiver. The apertures formed were up to $95-\lambda$ in length with temporal processing gains approaching 0.75 of the theoretical limit.

Pusone and Lloyd [1985] presented results on the degradation of passive SAS beamforming, as influenced by coherence time, random anplitude and phase fluctuations, and deterministic and random positioning errors of the synthesized array. The results provided guidelines for the design of a passive SAS.

Carey [1988] discussed the results of the 1984 (above) passive experimental data, the results of Fizzerald [1976], and the results of Williams [1976], which all indicate that passive, synthetic apertures may be formed by coherent summation as long as the synthesized array is shorter than the available coherence length and that the processing time is less than the temporal coherence length. Comparisons between conventional and synthetic arrays formed with either the same number of hydrophones or with the same effective aperture length but with a different number of hydrophones, showed an advantage for the synthetic array when the spatial processing gain is greater than the loss in integration gain.

Autrey [1988] discussed the concept of the passive synthetic array and showed it to be equivalent to a narrow-band processor, and also showed the gain to be determined by the bandwidth of the source signal. Autrey further stated that

"....the passive synthetic array has no new form of system gain and has no obvious new applications. This study has shown, however, that it should be possible to achieve improved bearing resolution in conventional systems incorporating narrow-band processors by executing a heading or speed change."

Carey [1989], commenting on Autrey's paper, had strong disagreements with Autrey's conclusion. This prompted a further set of exchanges between Autrey and Carey (both 1990) concerning passive synthetic arrays. The interested reader is urged to read Autrey's article and the "Letters to the Editor" which followed in the Journal of the Acoustical Society of America.

Yen and Carey [1989] published a paper from an earlier [1984] classified publication on the application of synthetic-aperture processing to towed array data. It was shown that significant increases in signal gain, improved angular resolution, and improved peak-to-sidelobe level ratios could be achievcd. The 
experiment used a geophysical streamer of 256 hydrophones with a $2.5 \mathrm{~m}$ spacing (637.5 $\mathrm{m}$ array), and was towed at a $226 \mathrm{~m}$ depth, approximately $195-$ to $250-\mathrm{km}$ from a moored source. The $175 \mathrm{~Hz}$ source was moored at a $300 \mathrm{~m}$ depth in $3200 \mathrm{~m}$ water. The results showed the passive SAS array had a coherent gain factor of 1.7 for synthesized array lengths from $192-10962-\mathrm{m}$, as formed from subaperture lengths from 5 to 80-m long, and over coherent processing times of less than 9 minutes (540 seconds).

Candy and Sullivan [1989] described a model-based passive ranging scheme coupled with a normalmode model and the use of a horizontal (towed) array, and showed that the model parameters need not be known a priori to find a solution. An example shown in the paper requires an array at least $500 \mathrm{~m}$ long which was considered unreasonable for the example water depth of $100 \mathrm{~m}$. The authors then say that "for a signal with sufficient stationarity, the wavenumber estimates could be made with a synthetic aperture traced out by a much shorter array or other model-based technique. In this regard, recent work on a new approach to passive synthetic aperture processing has been quite promising."

The 'new approach' mentioned by Candy and Sullivan was published by Stergiopoulos and Sullivan [1989] one month later, and showed a method of extending towed array measurements. The concept was based on what the authors referred to as an "overlap correlator," which provides a phase correction by correlating successive and overlapping array samples of the acoustic signal received by a moving towed array. This method can then coherently combine acoustic signals by making the proper compensation which corrects for considerable fluctuations in phase irregularities in the array tow path as well as irregularities in amplitude experienced during the coherent integration time. This is a form of autofocusing in synthetic aperture processing.

Stergiopoulos [1989] showed the optimum bearing resolution for a moving towed array and the extension of its physical aperture. In a companion paper presented at the same meeting, Sullivan and Stergiopoulos [1989] described a new method for passive SAS prosessing by using the existing aperture (i.e. the aperture of the real array of hydrophones) to estimate a phase correction factor for the successive measurement, and therefore avoids knowing a priori the source frequency. Results using artificial and real data demonstrated that the method outperforms previous methods. This work was further extended by Edelson and Sullivan [1990] and was also demonstrated in resolving multiple sources using simulated data.

Urban and Stergiopoulos [1990] showed experimental results of a long (100 $\lambda)$ passive synthetic aperture in an ocean where the spatial and temporal coherence properties were known. They concluded that for a multitarget environment, the processing must correct for defocusing due to relative source-array motion, and that the plane wave assumption is invalid for close (nearfield) targets. 


\subsection{Contributions of This Thesis to the Ficld of SAS}

There are several important contributions in this thesis to the field of synthetic apertures which could apply to both radar and sonar applications.

The first contribution, and the major one of the thesis, is the computer model. It differs from previous synthetic aperture sonar models because it includes all the relevant platform and sonar parameters, a temporally and spatially varying stratified environment, and a v ride variety of processing options. As such, it is useful as a tool for studying the ocean, platform, and signal processing effects on synthetic aperture sonar (hence the title of the thesis), and may also be used to study SAR after suitable modification of the model parameters.

The second contribution is that the explanation of synthetic apertures is attempted from geometrical means by the use of copious figures, and intentionally avoids integral mathematic notation. I have found that nearly all of the SAR and SAS literature emphasizes equation-based descriptions, and with few accompanying, useful, figures. My father describes this as 'overly elaborated for the sake of academic sophistication.' Since pictures (figures) can convey ideas far better than words, I have attempted to do so where possible.

The ocean effects on SAS imaging performance include multipath, deep ocean refraction, and medium stability. Short-range multipath involving the surface and the bottom is shown to have a small influence on imagery due to the rough, time-varying air-water interface at the surface, and the absorptive bottom.

Deep-ocean SAS imagery using a 'wide' beam (high resolution) synthetic aperture sonar requires a good estimate for the sound speed profile to yield a sharp SAS image. 'Narrow' beam synthetic aperture sonars do not suffer this limitation; they image well in spite of the poor sound speed estimate, but the image is frequently displaced from its true location. This suggests that there may be an optimum beamwidth which will give a gond azimuthal resolution and yet still be insensitive to sound speed mismatch.

The coherence time for the ocean medium is surveyed from the literature and a set of dimensionless numbers are given, which are reference standards among the different SAS experiments. From these numbers, the coherence time for a proposed SAS system may be crudely estimated based on the wavelength, the acoustic path length, and the variance. The computer model, meanwhile showed that images formed 
using data taken over time exceeding the coherence time shows target image degradation, but the target image nevertheless persists.

The motion of the sonar platform must be known to within $\lambda / 4$ (during round-trip) for sharp images, but this requirement may be relaxed somewhat if a reduction in resolution is accepted.

The influence of the natural amplitude taper on the level of the first synthetic aperture sidelobe is discussed in detail and shown to vary from $-6.6 \mathrm{~dB}$ to $-20 \mathrm{~dB}$ depending on the processing assumptions. The sidelobe levels for synthetic apertures using the natural taper of the real aperture beampattem have been briefly discussed in the SAR and SAS literature, but without describing the exact bounds on the level.

A major contribution in this thesis is that synthetic apertures sampled at the Nyquist rate must not be assumed to be free from azimuthal alias lobes (this assumption has been frequently made in the SAS literature; the SAR literaure has a few studies on azimuthal ambiguities). This thesis shows that apertures spatially sampled at the Nyquist rate must not use the full length across the $-3 \mathrm{~dB}$ mainlobe, otherwise there will be a significant alias contribution in the image space. Instead, the full length across the $-3 \mathrm{~dB}$ mainlobe width should be divided into several sub-apertures; forming an image from one sub-aperture will show that the alias lobes are reduced (or indistinguishable from the sidelobe structure). Images may then be formed from the rest of the sub-apertures, and the ensemble of images may then be averaged (this averaging of images from several sub-apertures of a full aperture is called multilook). This also implies that the multilook technique in SAR used to reduce speckle and improve image quality (but reduces the actual resolution) may in fact be as worthwhile for alias lobe suppression as it is for speckle reduction.

A further contribution conceming Nyquist-operated synthetic aperture sonars is that too much signal bandwidth does not lead to minimum alias lobes. This suggests that synthetic arrays which intend to be very broadband should either be: (1) very spatially oversampled, or (2) very spatially undersampled.

Another important contribution to the field of SAS is that a "ballnark" formula is developed for determining the level of alias lobe smear (in $\mathrm{dB}$ ) for a broadband undersampled synthetic aperture sonar. This contrasts with some previous work which showed bandwidth requirements for smearing, but did not establish the level of the smearing relative to the image value for the target.

Broadband undersampled apertures are also shown to be more effective at alias lobe suppression when the full sonar mainlobe (null-to-null) is used, instead of the customary $-3 \mathrm{~dB}$ to $-3 \mathrm{~dB}$ main lobe. 


\subsection{Thesis Organization}

This thesis is organized into six chapters, and several appendices. Chapter 1 is the introduction and provides a historical and summary overview of synthetic aperture radar (the parent) and synthetic aperture sonar (the offspring). The previous work in SAS is shown, and is divided into low-frequency active SAS (below $1 \mathrm{MHz}$ ), high-frequency active SAS (above $1 \mathrm{MHz}$ ), and passive SAS. The contributions of the thesis are shown, and the thesis organization is shown in this section.

Chapter 2 is the longest chapter in the thesis, and it relies on a large number of figures to accompany the descriptions in the text. It first gives the operating principles of a real aperture and compares them to the principles for the synthetic aperture. The premise is that if one can understand a real aperture, then one may understand the synthetic aperture by pure extension of the same basic principles. The "image" which results from an aperture synthesis is shown to be a 2-D map of the magnitude of target reflectivity. Three operating modes of synthetic apertures are discussed: focused, unfocused, and the spotlight; the along-track resolution for each is shown. A detailed transform-pair approach is then used to describe the sampling requirements of the synthetic aperture, and the relation between sampling, the pulse rate, and the maximum unambiguous range are shown. The use of broadband signals is discussed in detail, and then the chapter concludes with several signal processing options.

Chapter 3 details the approach taken in computer modeling the environment and in forming synthetic aperture images. The two programs used for this thesis are described, as well as the program capabilities and modeling assumptions.

Chapter 4 discusses the influences of the acoustical environment on SAS, and makes a contrast to the radar environment for SAR. This chapter also includes a summary of the previous work and SAS-related experiments on medium stability.

Chapter 5 provides example SAS imaging from the computer model for a variety of operating and environmental conditions, covering the scope of the ocean, platform, and signal processing effects on acoustical imaging. This chapter offers the result-oriented thrust in the thesis, in contrast to Chapter 2 which is predominantly theory.

Chapter 6 offers the summary of the thesis, the conclusions, and the direction for future work in SAS. 
The thesis is concluded by several appendices and the bibliography. 
This page left intentionally blank. 


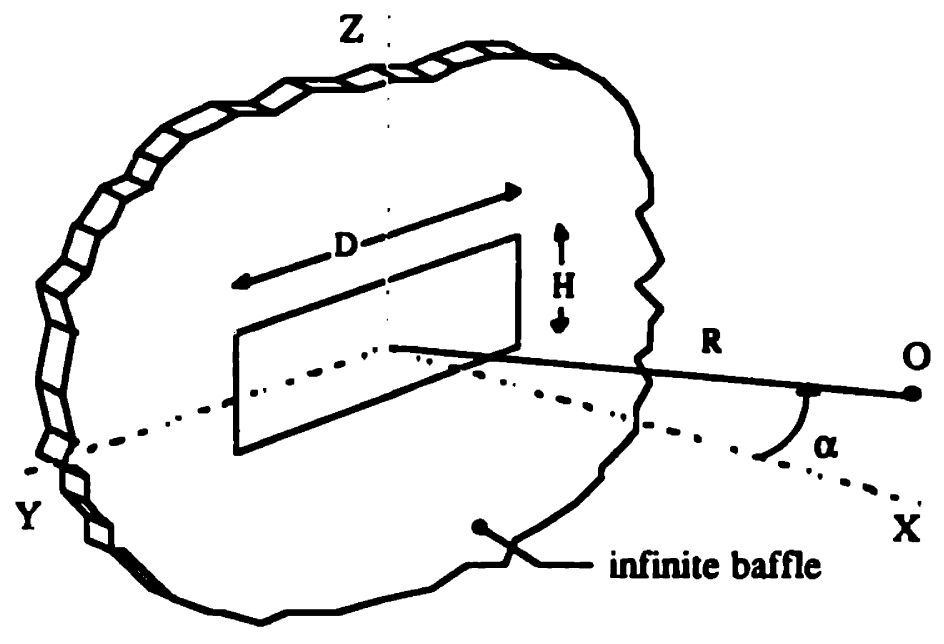

A. - Perspective view

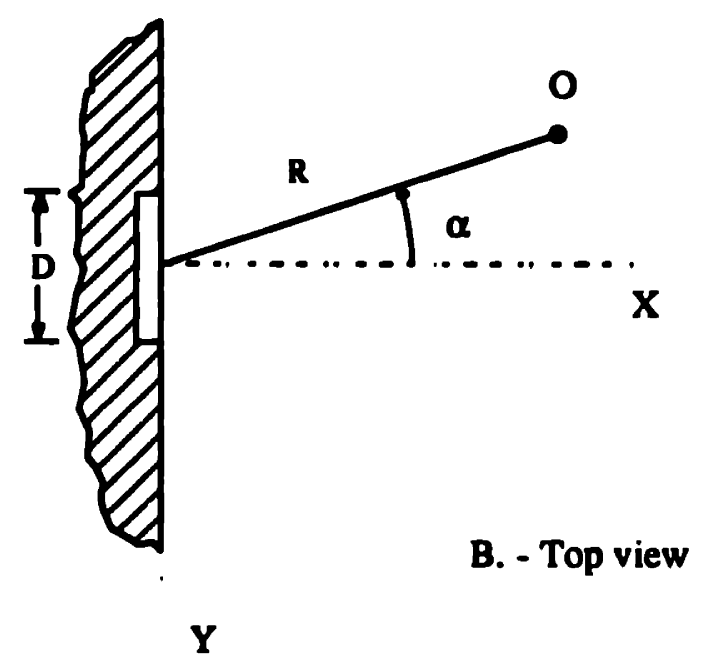

Figure 2.1 - Rectangular Piston in an Infinite Baffle 


\section{Chapter 2}

\section{Synthetic Aperture Sonar (SAS) Principles}

In this chapter the principle of forming a large synthetic aperture from the sequential use of a small real aperture is given. Real apertures are reviewed and a comparison is then made to the synthetic aperture (or synthetic array). The theory of synthetic apertures is shown by geometry, and several modes of SAS are discussed. The requirements of sampling and the level of the sidelobes are discussed, and the relaxation of sampling requirements by the use of broadband signals is cieveloped. Several processing options are then shown.

\subsection{Real Apertures (Arrays) versus Synthetic Apertures (Arrays)}

The contrast between a real aperture and a synthetic aperture is made here. Details of real apertures and arrays, particularly for use in high resolution sonar imaging are given by Sutton [1979] and Berktay [1985]. and a treatment of lenseless acoustical imaging is given by Kino [1987].

\subsubsection{Real Apertures}

Consider the real aperture (or real antenna) of a rectangular piston shown in Figure 2.1. The piston is installed in a large (infinite) baffle, which makes the analysis simpler by removing the effects of diffraction from baffle boundaries such as edges, and the analysis is confined only to the sound radiation from the piston in a direction normal to the yz-plane. The piston is considered rigid and moves with uniform velocity. Though the piston is not an array (an array implies more than one transmitting or receiving element) it has the directional characteristics shown in Appendix A, repeated here as

$$
B(\alpha)=\frac{\sin (x)}{x} \equiv \operatorname{sinc}(x)
$$

where $x=K D / 2$; ( $x$ is a dummy variable and not to be confused with the $x$-axis)

$K=k \sin (\alpha)$

$k=\omega / c=2 \pi f / c=2 \pi / \lambda$

$D=$ the $y$-axis length in Fig. 2.1 
and for ranges greater than $D^{2} \wedge$ (i.e. the far field approximation).

$B(\alpha)$ is called the one-way beam pattem function in the xy-plane, and depends on the angle $\alpha$, which is measured from the main radiation axis (shown as the $x$-axis). The one-way aspect of equation 2.1 is due to the $B(\alpha)$ being measured in the out-direction from the piston to the observer point $O$. The two-way beam pautem function is the convolution of the out-direction pattem function with the back-direction pattem function, and by way of Appendix $A$, was shown to be $B^{2}(\alpha)$ because the out- and back-direction patterns are the same (for the monostatic case where a single element or single array is used for both transmit and receive).

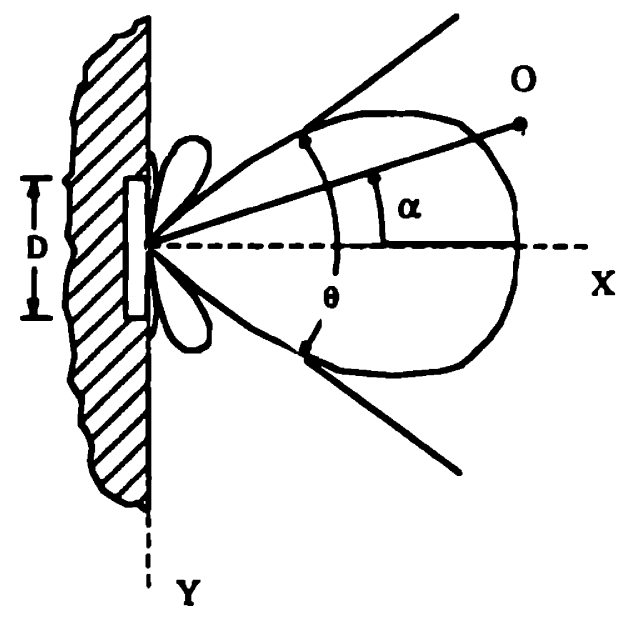

Figure 2.2 - Top view, rectangular piston in an infinite baffle with example radiation pattern (beam pattern). 

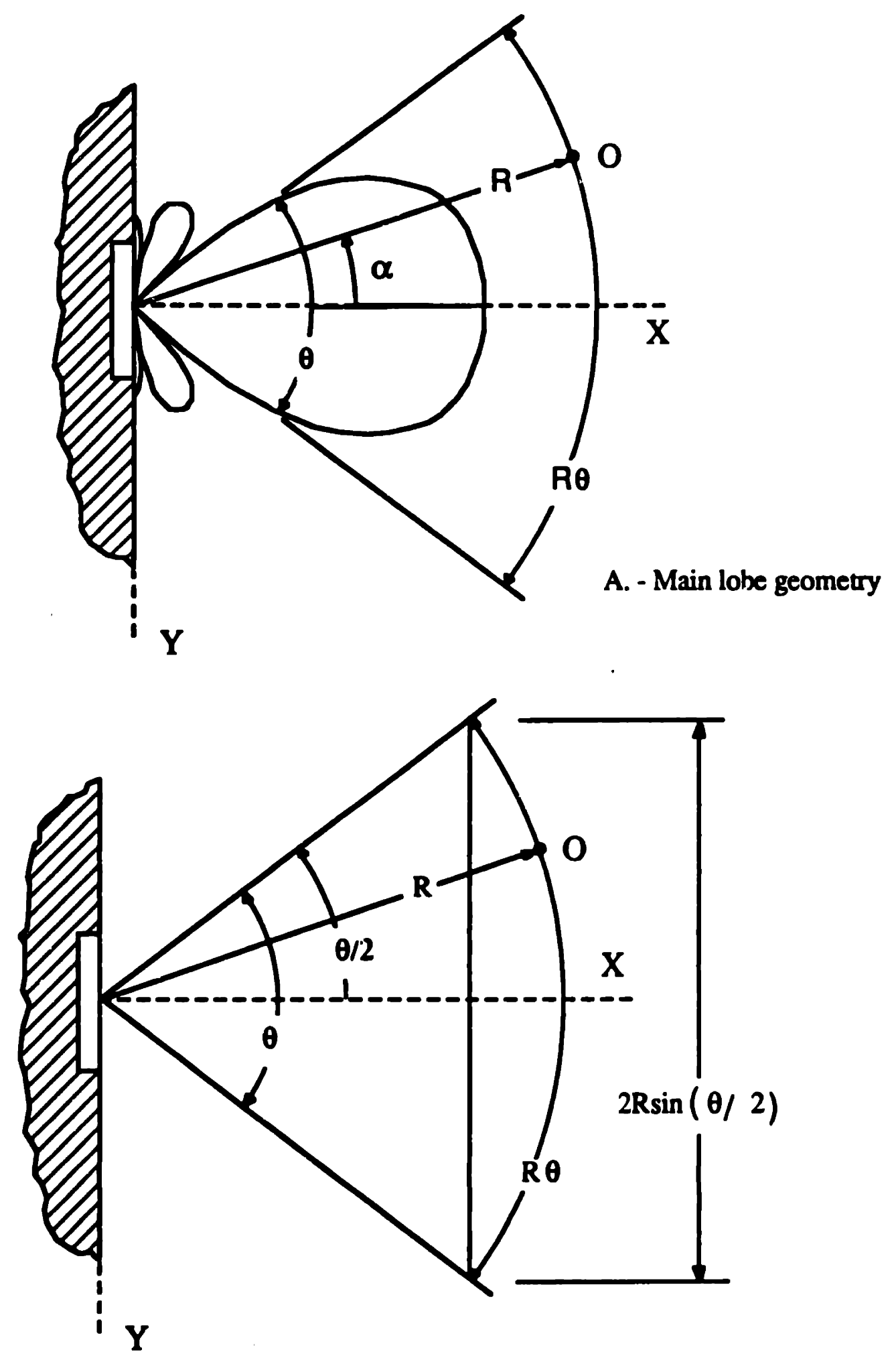

B. - Main lobe dimensions

Fig. 2.3 - Rectangular piston main lobe, geometry \& dimensions 
Appendix $\mathrm{A}$ also shows that the $-3 \mathrm{~dB}$ width of the main radiation lobe (i.e. the mainlobe) is given by

$$
\theta_{\text {mainlobe }}=\theta_{\mathrm{ml}} \approx \lambda / \mathrm{D} \quad \text { (in the xy-plane) }
$$

where $\lambda=$ wavelength of sound radiation $(m)$

$$
D=\text { sonar } y \text {-axis dimension }(m) \text {. }
$$

For now we confine our interest to the horizontal (xy) plane radiation, so we redraw Figure 2.1 from a section view in the $x y$-plane and include the $-3 \mathrm{~dB}$ mainlobe and several sidelobes, as shown in Figure 2.2. The acoustical y-direction resolution of the piston is determined by $\theta_{\mathrm{ml}}$ and the range from the piston, and the $x$-direction resolution is determined by the characteristics of the transmit signal (see Appendix B). Resolution is the ability to correctly determine the size of an object located within the view of the sonar, the sonar being the rectangular piston. For our purposes, an object illuminated (or more specifically insonified) by sound radiation from the piston will be resolved correctly for objects larger than

$$
y \text {-resolution } \equiv \rho_{y}=2 R \sin \left(\frac{\theta_{\mathrm{ml}}}{2}\right)
$$

which may be explained by the geometry shown in Figure 2.3. The resolution is dependent on both the angular width of the main lobe $\theta_{\mathrm{ml}}$, and the range $R$ because the $y$-direction width of the main lobe increases with $R$. For small values of $\theta_{\mathrm{ml}}$

$$
\sin \left(\frac{\theta_{m l}}{2}\right) \cong \frac{\theta_{m l}}{2}
$$

and so the $y$-direction resolution may be given by

$$
\text { y-resolution }=\rho_{\mathrm{y}} \cong \mathrm{R} \theta_{\mathrm{ml}}
$$

As an illustration, consider a typical side scan sonar platform "fish" [e.g. Klein, 1982] with the topview geometry and targets as shown in Figure 2.4-A. The sonar travels above the bottom, but has a distance to the bottom that is small compared to the range of interest. This sonar is specifically designed to transmit and receive from the side and is usually called a "side looking" or "side scanning" sonar. Three rectangular targets (A, B, and C) are placed on the bottom, and all have the same height from the bottom and all are at a nominal range $R_{0}$ from the $y$-axis. The bottom is nearly flat, and the three targets are the 


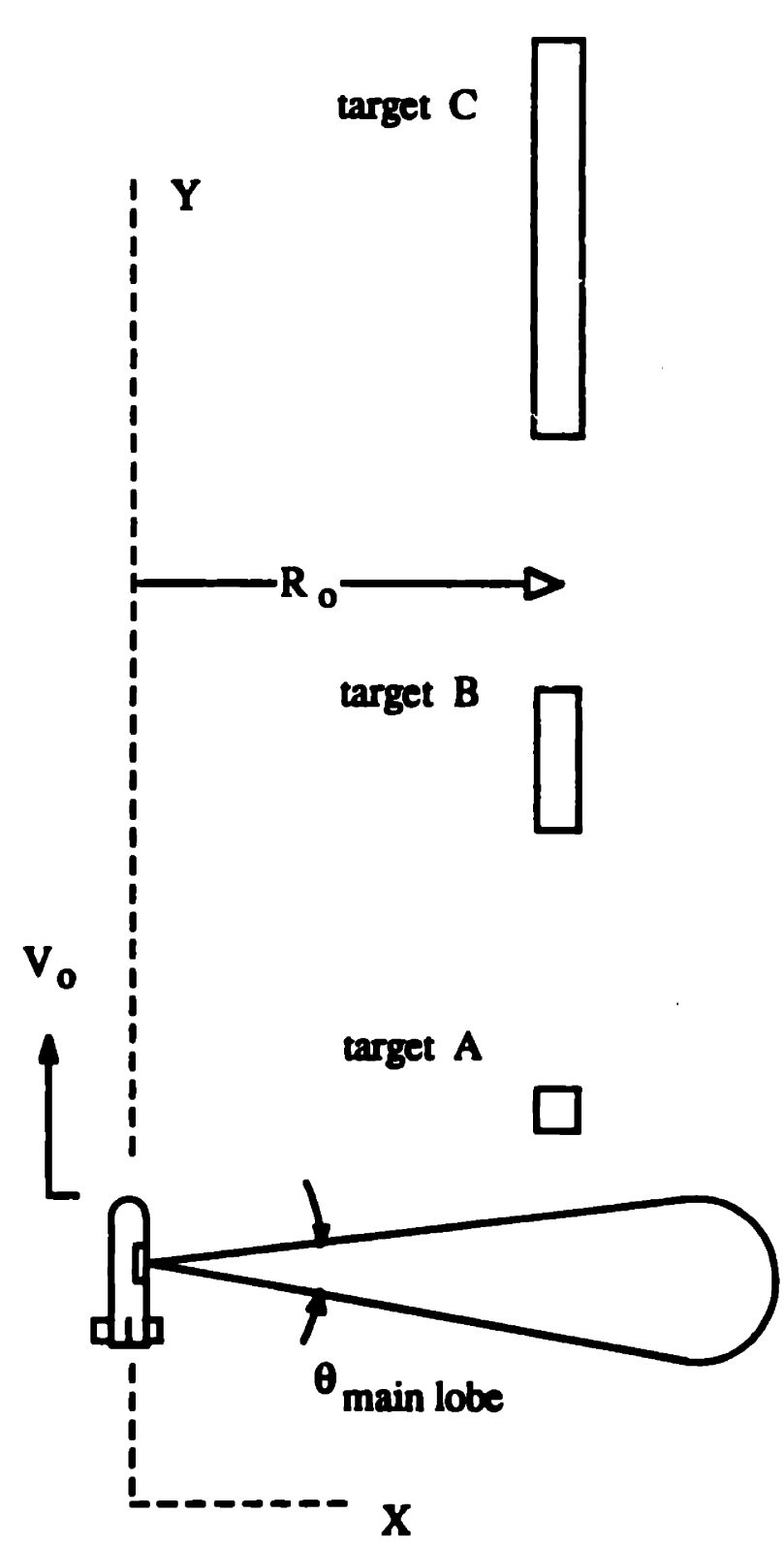

A. - Geometry

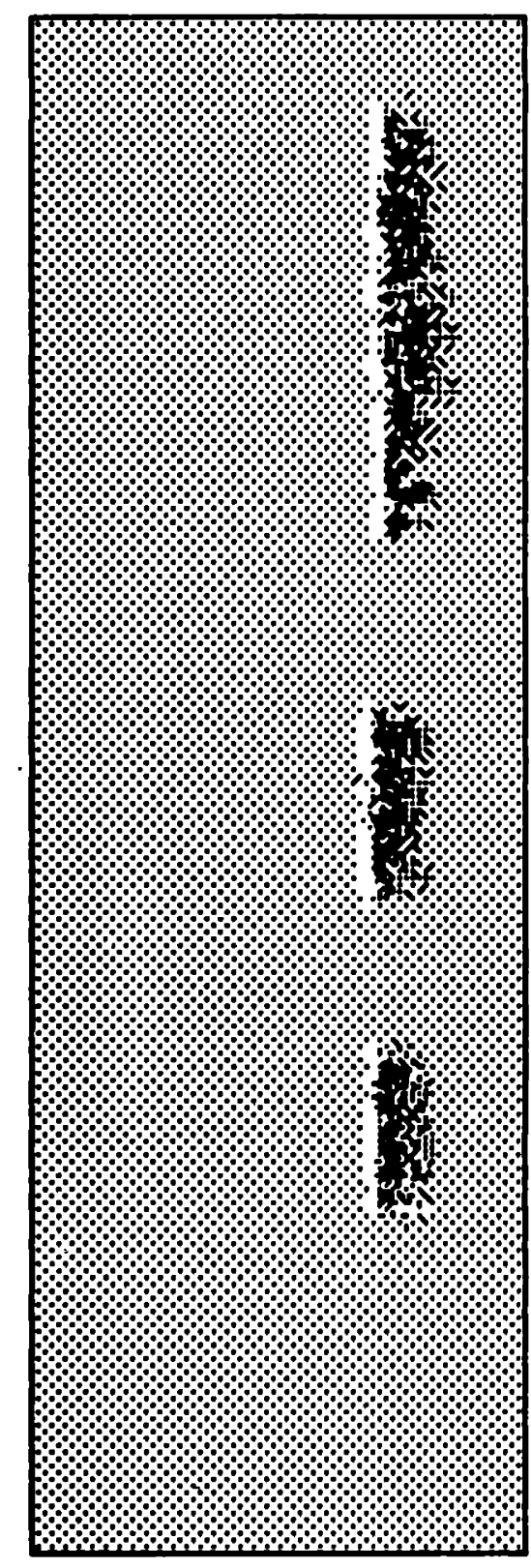

B. - Image display

Fig. 2.4 - Side scan sonar. Geometry and image display. 
only important features in the area. The sonar "fish" is towed along the $y$-axis, at speed $V_{0}$ from the surface by a cable, and it transmits and receives from rectangular pistons, one on each side of the fish body (only one side is shown for clarity). The width of the $-3 \mathrm{~dB}$ mainlobe of the sonar is indicated, and the projector-hydrophone piston transducer is directed at broadside (at a right angle to the direction of travel).

Figure 2.4-B shows the image resulting from the side scanning of the sonar fish as it passed all three targets. Note that target $\mathrm{C}$, which was considerably larger than the width of the sonar mainlobe at range $R_{0}$, is well resolved (the image closely resembles the size of the actual target). The medium-sized target $B$ is also resolved though not as well as $\mathbf{C}$, and target $\mathbf{A}$ is smeared because it incorrectly appears to be as large as the image of B. Also note that the gray-scale used in this image has strong reflecting targets as white, and acoustic shadows as black. This choice is often reversed, with acoustical shadows appearing white and strong reflecting targets appearing black.

This illustrates the $y$-direction resolution of a rectangular piston, as demonstrated by a side scanning sonar example. The y-direction resolution is similar for a real aperture array of discrete sonar elements. Figure 2.5 shows an example of a real aperture array of 5 piston elements. Each element is mounted in a large baffle (for the same reasons as the single rectangular piston) in the yz-plane and arranged along a line (the $y$-axis). The nominal length of the array is $L$, as given by

$$
\mathbf{L}=(\mathbf{N}-1) \mathbf{p}
$$

$$
\begin{aligned}
\text { where } N & =\text { the number of elements } \\
\qquad & =\text { center-to-center (inter-element) spacing, (m). }
\end{aligned}
$$

Each sonar element has a y-direction length $D$, and the array is centered on the xyz origin (for convenience). For the array, it may be shown that the $y$-direction resolution is

$$
\rho_{y}=2 R \sin \left(\frac{\theta_{m l}}{2}\right) \text {. }
$$

or with the small angle approximation of equation 2.4 , the $y$-direction resolution is

$$
\rho_{\mathbf{y}} \cong \mathbf{R} \theta_{\mathrm{ml}} \text {, }
$$

where $\rho_{y}=y$-direction resolution $(m), R=$ broadside range $(m)$, and

$$
\theta_{\mathrm{ml}}=-3 \mathrm{~dB} \text { mainlobe beamwidh (radian measure) } \approx \lambda / \mathrm{L} \text {. }
$$




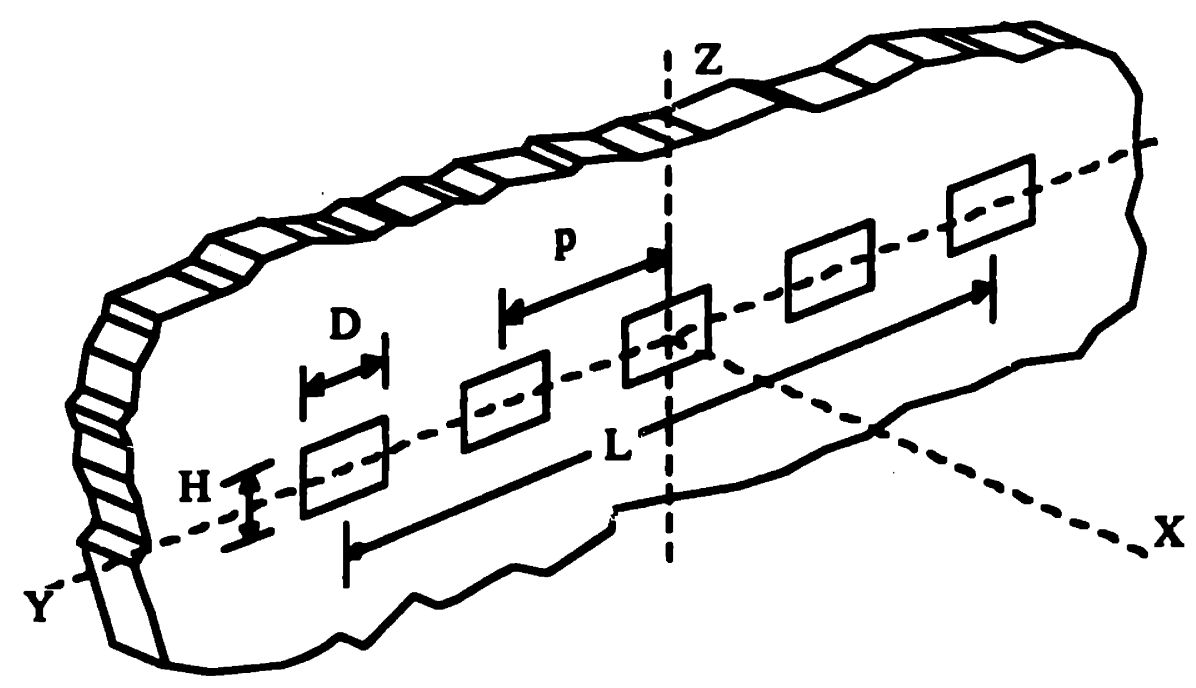

A. - Perspective view

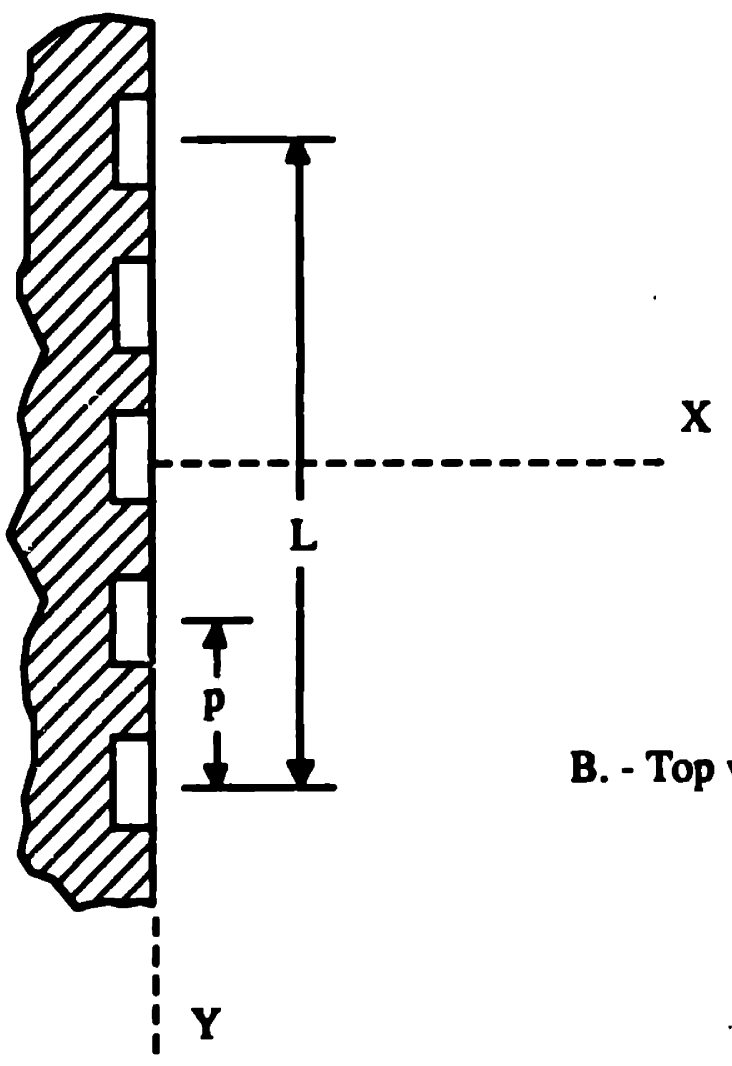

Fig. 2.5 - Real Aperture Array

45 
Both (2.7a) and (2.7b) have the same form as (2.2). This shows that the y-direction resolving ability of an aperture, whether it is a single (continuous length $\mathrm{L}$ ) element or an array of elements (a discrete sampling of a continuous length $L$ ), is proportional to the range $R$, wavelength $\lambda$, and inversely proportional to the characteristic length L. The sampled array, has however, specific requirements on sampling to avoid aliasing lobes (lobes which exactly resemble the main lobe that are spaced at regular intervals). The requirements of sampling for the discrete array of elements may be found in Dyer [1989].

These examples show the y-direction resolution for real apertures, either as a single element, or as an array of elements. Two other features of real apertures should be mentioned before discussing the synthetic array. The first feature is the type of transmit signal and how it is used in finding range. The second feature is focusing.

\subsubsection{Incoherent and Coherent Sonar}

Sonars (and radars) may be classified as either incoherent (also known as noncoherent) and coherent. In the incoherent sonar, only the envelope of an echo is important and the phase of the underlying acoustic signal is ignored. For example, the elapsed time after transmission for any single, large echo determines the range of a target from the relation

$$
\text { range }=c \frac{T_{\text {elapsed }}}{2} \text {, }
$$

where $T_{\text {elapsed }}$ is the round trip elapsed time,

c is the sound speed,

and the decision on target/no target depends on the magnitude of the echo. Large echoes are easily discemable from the threshold of background noise, but weak echoes are not. Threshold detection of this type shows both the simplicity and difficulty with the incoherent sonar. Targets are located when the magnitude (absolute value) of an echo exceeds a pre-set threshold, but weak echoes may not exceed the threshold and could therefore be overlooked. Furthermore, overlapping echoes from two or more targets may appear as a single, large target, which causes some confusion (or ambiguity, an overused and seldom defined description in radar and sonar processing). Other rules for making the decision (is there a target ?) besides threshold detection may also be used. The important feature is that the incoherent sonar uses only the magnitude of the retum echoes in the received signal processing and does not use the phase. 
The coherent sonar uses both the magnitude and phase of the echo return during signal processing, and usually compares a stored replica of the transmit signal with the received signal. This is called replica correlation, and is equivalently a form of matched filtering. The details of how this is performed are shown in Appendix B.

\subsubsection{Focused Sonar}

The second feature is focusing. The discrete array (length $\mathrm{L}$ ) of elements has an advantage over a continuous (length $L$ ) element in that each array element may be activated at separate time to cause the transmit signals from all the elements to arrive at a single point (the focus) simultaneously. A similar procedure may also be used for reception. Thus the array has the ability to be focused in both transmit and receive, and this is usually implemented by electronic means and referred to as electronically timed or phased arrays. The analog for a single focused element uses either a single continuous curved element, or uses the combination of a single straight element and a focusing lens.

Familiarity with the concepts of apertures (either of the continuous or discrete variety), noncoherent and coherent processing, and electronically timed/phased arrays are useful for thorough understanding of most applications of sonar and radar. These concepts become key when trying to understand the notion of the synthetic aperture or synthetic array, which will now be discussed.

\subsubsection{Synthetic Apertures (or Synthetic Arrays)}

The simplest way to describe synthetic apertures is by geometry. To begin, consider a small real aperture sonar (with element length $\mathrm{D}$ ) having an usually wide $-3 \mathrm{~dB}$ mainlobe beam, as shown in Figure 2.6. This sonar would be, by itself, a poor imaging sonar because the mainlobe is too wide for useful imaging. For purposes of illustration, let's say that the sonar is fixed at the $x y$ origin and just above the bottom, and that there is a single point target on the bottom, located at $x_{0}, y_{0}$, which is just within the view of the mainlobe. [Note: Placing the sonar just above the bottom allows us to assume that the slant range is effectively the same as the ground range, for distant targets. This turns the 3-D geometry problem into 2-D geometry, which is easier to illustrate in the Figures. For the remainder of this thesis, the term range implicitly means slant range.] A sonar pulse (which we do not specify as yet) would travel out within the mainlobe, strike the target, and return an echo which is recorded in a time series. The echo would be received at the sonar in the time series at time 


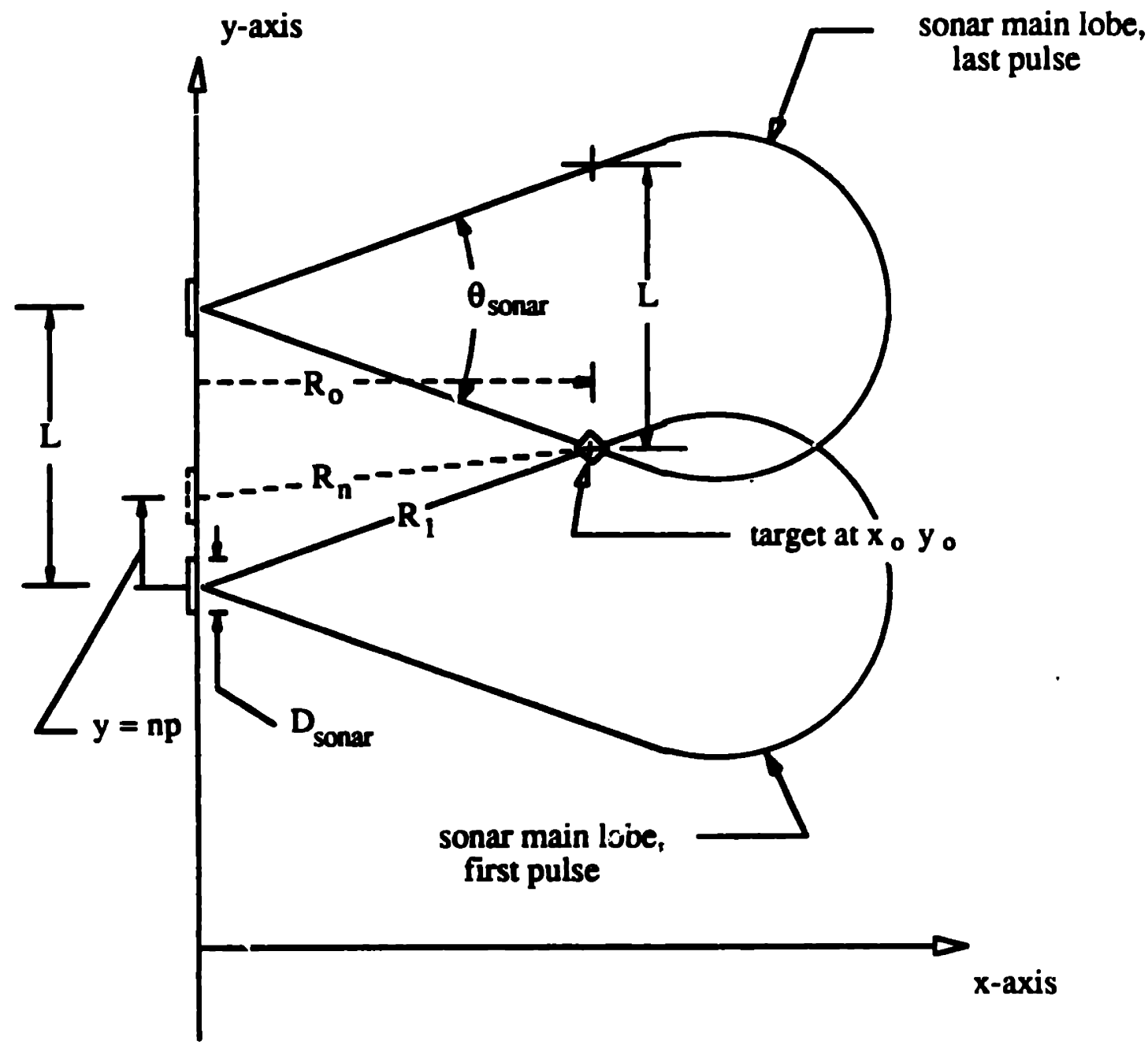

Figure 2.6 - Synthetic Aperture via Geometry 


$$
\begin{gathered}
t_{1}=\frac{2 R_{1}}{c} \\
\text { where } R_{1}=\sqrt{\left(x_{0}-0\right)^{2}+\left(y_{0}-0\right)^{2}}
\end{gathered}
$$

We now move the sonar platform a distance $p$ to a new location along the $y$-axis, and repeat the pulseecho experiment. In radar, the $x$ - and $y$-directions have specific names which will be used here as well. The direction of platform movement is called both the along-track direction and the azimuth direction. The orthogonal (normal to along-track) direction is called both the cross-track direction and the range direction. We note a new echo time for the second pulse as

$$
\begin{gathered}
t_{2}=\frac{2 R_{2}}{c}, \\
\text { where } R_{2}=\sqrt{\left(x_{0}-0\right)^{2}+\left(y_{0}-p\right)^{2}} .
\end{gathered}
$$

We then sequentially repeat the experiment $\mathrm{N}$-times until the target reaches the other side of the sonar $-3 \mathrm{~dB}$ mainlobe. The extreme positions of the sonar (entering the mainlobe, and leaving the mainlobe) are also shown in Figure 2.6. We may also write a general form for the echo for a target at $\mathrm{x}_{0}, \mathrm{y}_{0}$ :

$$
\begin{gathered}
\qquad t_{n}=\frac{2 R_{n}}{c} \text {. } \\
\text { where } R_{n}=\sqrt{\left(x_{0}-0\right)^{2}+\left(y_{0}-n p\right)^{2}} \\
y=n p, \\
\text { and } n=1,2,3 \ldots . . . N \text {. }
\end{gathered}
$$

Physically, we assume that the only significant echoes from the target occur when the target is within the $-3 \mathrm{~dB}$ mainlobe of the sonar. There will, of course, be echoes reaching the sonar for sonar positions which transmit and receive outside the $-3 \mathrm{~dB}$ mainlobe, and in the side lobes. For now, we ignore these influences and restrict our attention to only the $-3 \mathrm{~dB}$ mainlobe contribution.

The important features of Figure 2.6 are the sonar positions ( $x=0, y=n p)$ and the $R_{n}$. We also define $\mathbf{R}_{\mathbf{0}}$, which is the range of the target from the sonar at the point of closest approach, $\mathbf{R}_{\mathbf{0}}$ is also customarily called the bioadside range because it is located normal to the broad side (the long side) of the sonar platform. We create a new geometrical figure, Fig. 2.7, including these features, and find that we 


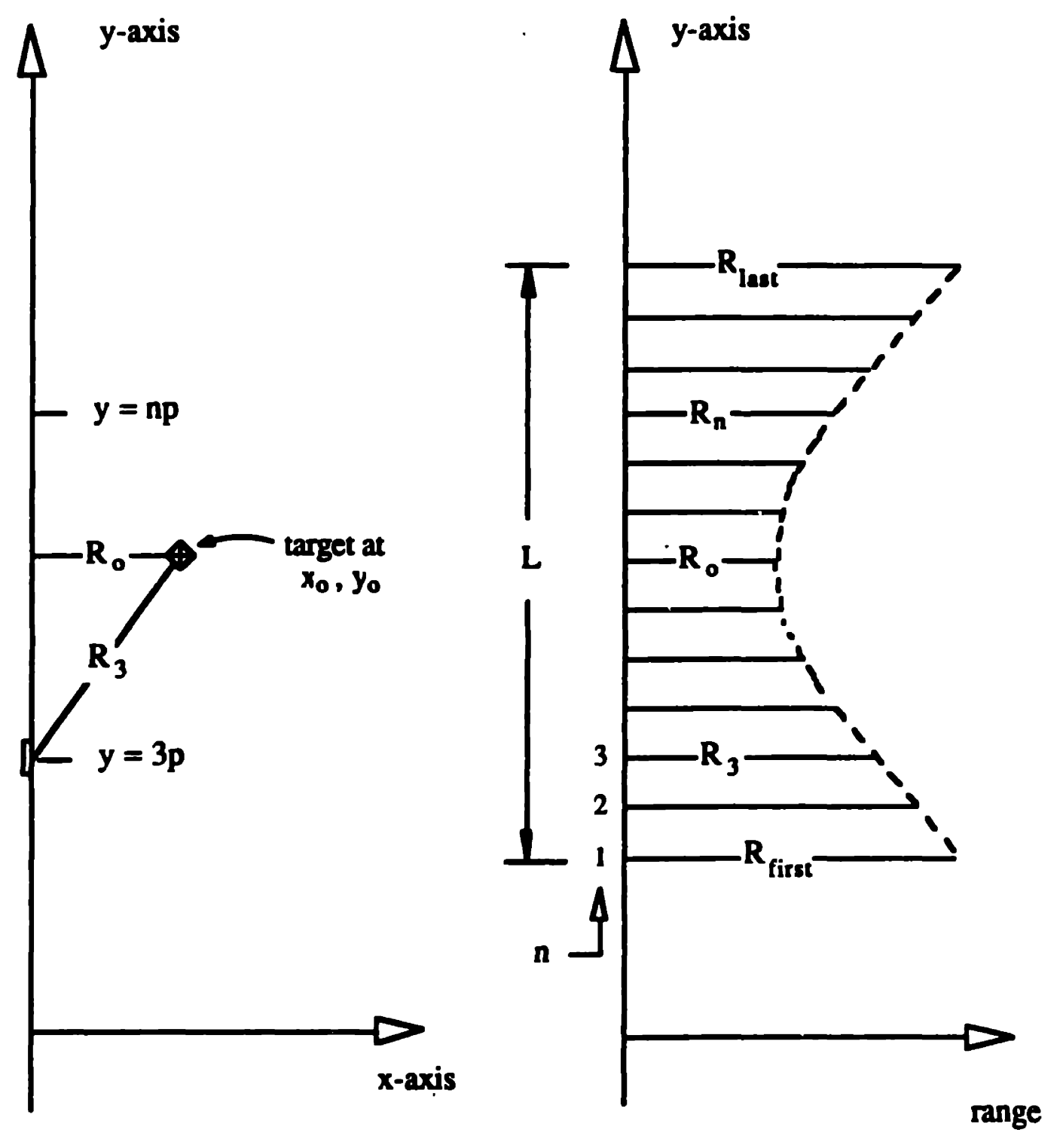

Figure 2.7 - Range Migration Hyperbola of Target at $x_{0}, y_{0}$ 
have a map of the migration of the target as it passes through the view of the sonar $-3 \mathrm{~dB}$ mainlobe. This is called range migration. On the whole, it is a hyperbolic arc, but locally in the broadside region it looks much like a parabola. The approximation to a parabola is one that is frequently made in synthetic aperture radar.

An important aspect of the geometry shown in Figure 2.7 is that it always looks the same for any target, no matter what its broadside range is. An example shown in Figure 2.8 has three point targets at three different broadside ranges. The key feature is that for targets that are at small broadside ranges, the length

$$
\mathbf{L}=\mathbf{N p} \text {, }
$$

is commensurately small. For targets at large broadside ranges, the length $\mathrm{L}=\mathrm{Np}$ is appropriately larger. The only difference then, is one of scale. Targets far away (large $R_{0}$ ) have large $L=N p$; targets close (small $R_{o}$ ) have small $L=N p$. What is the same for all these cases is that the ratio of $R_{0}$ to $L$ is constant, because the width of the $-3 \mathrm{~dB}$ mainlobe is constant for all three cases (the mainlobe width is independent of the target range). This is the similar triangles argument, and will be recalled shorty when discussing along-track resolution.

Now if we had saved all $\mathbf{N}$ time series we would have a collection of echoes for all targets within the view of the sonar, as the sonar was sequentially moved from the origin, to $y=N p$, at step distance $p$. For our simple case, we only had a single target located at $x_{0}, y_{0}$. An example representation of each time series (or time record) having a single echo, and showing the range migration of the echo across the ensemble of records is shown in Figure 2.9. The transmit signal is shown for convenience as an FM (frequency modulation) sweep, or chirp. Note also that Figure 2.9 shows each $\mathrm{n}$-th time series referenced to the start time of the $n$-th pulse.

The concept of a synthetic aperture or a synthetic array comes from the knowledge that a fixed target within the view of a sonar (or radar) will exhibit a range migration as the sonar (or radar) platform travels a straight line path, and similarly the echo from that target will exhibit a time-of-flight migration. By recognizing the geometry of range migration, it is possible to constructively add the ensemble echoes returned from one target, from sequential time series. This processing step is focusing, since it attempts to add together only echoes, from separate pulses, which were returned from the same target located at $x_{0}, y_{0}$. Since the data used in this focusing step is taken over the distance $L=N p$, then $L$ is the length of the synthetic aperture or synthetic array. 

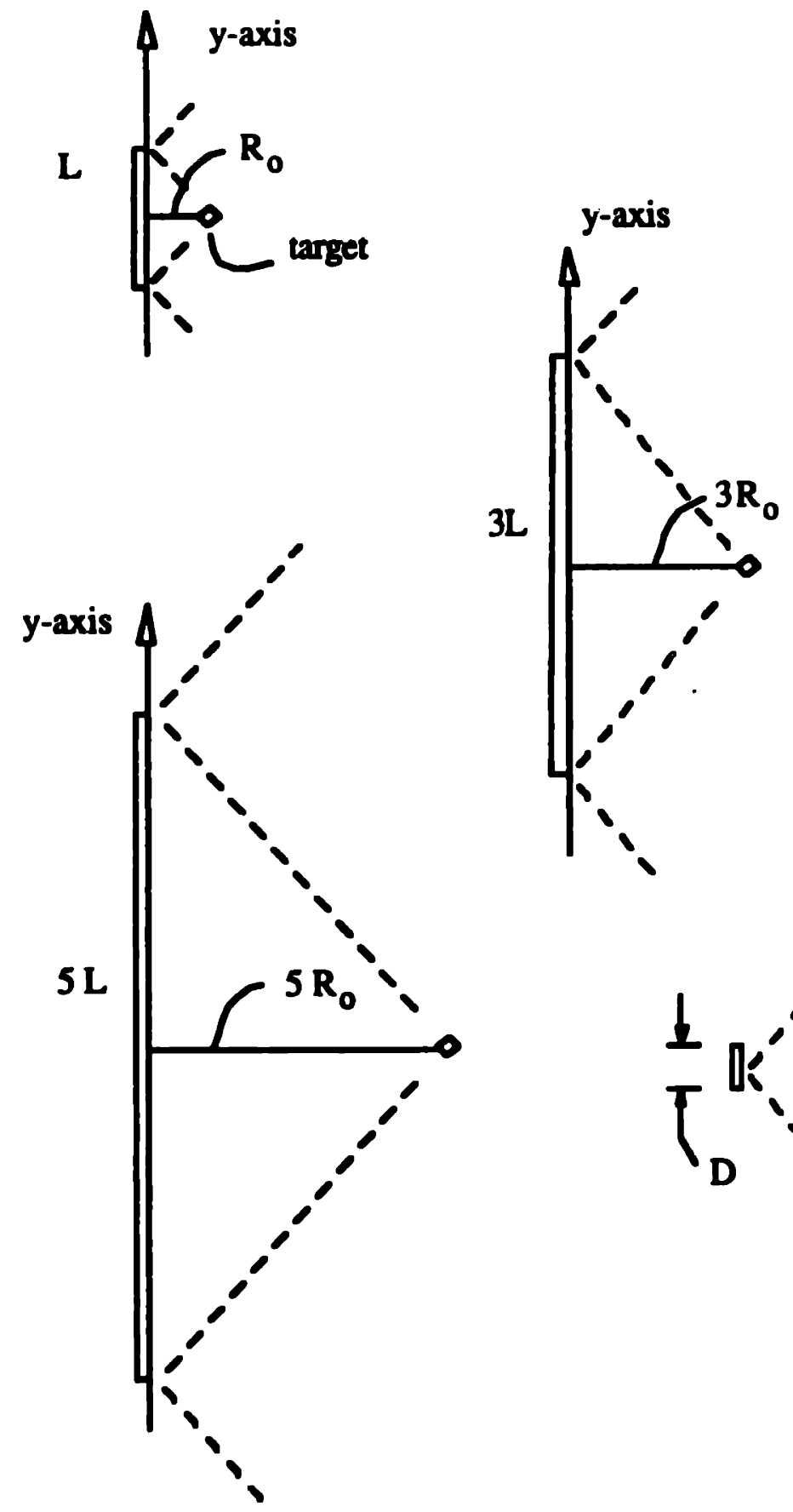

Figure 2.8 - Similar Triangles Argument 


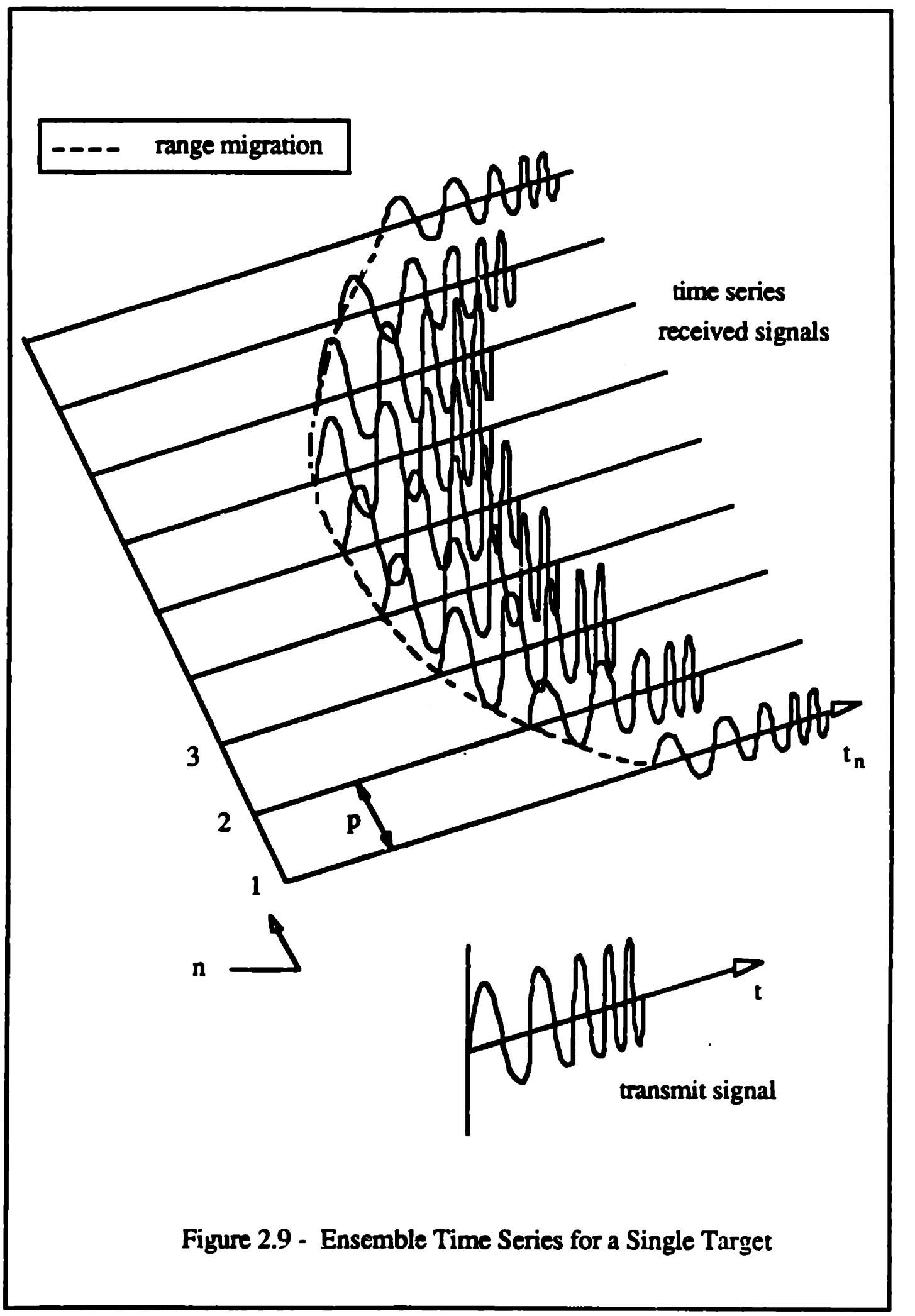


It is now obvious that the real aperture sonar, with sonar length $D$, which by itself makes a poor imaging sonar, can be used to collect a number of sequential echoes, to form a range migration history. The ensemble of these echo histories may then be combined by signal processing means. The use of signal processing to focus echoes from an ensemble of sequential time records from a sonar/radar over a path distance $L$ is the notion of synthetic aperture.

In the above example, we have deliberately moved the sonar platform in distance steps of length $p$. There are actually examples where this discrete stop-and-go stepping of the sonar/radar platform, from one location to another, occurs. For the radar case, there are cxamples of ground penetrating radar that are used to find buried objects such as underground pipes [Liem and Davis, 1988]. The wavelengths required for useful ground penetration are, however, very long especially as compared to the radar transmitter. This tends to widen the main lobe of the transmitter and ruins any useful imaging capability. Using a shorter wavelength would make the transmitter more directional and therefore more useful as an imaging tool, but ground penetration is then lost. But focused aperture synthesis techniques allow the radar to both penetrate the ground, and give useful accuracy in locating objects. One sonar application of the stop-and-go aperture synthesis method is in ultrasonic non-destructive testing (NDT) of solids [Doctor et alia, 1986]. In this case, a wide beam ultrasound probe is sequentially moved along a baseline (the path of the sonar platform) and along the way it pulses and then stores the received echoes. The intended targets for this case are flaws in the material. Once all the pulse-echo data (i.e. the ensemble) is collected, the inage processing may be performed.

It is much more common in synthetic aperture methods to allow the platform to travel at a known speed $V_{o}$, where $V_{0}$ is usually constant, as the platform transits along a known path (usually a straight line). This is typical for airborne and spaceborne SAR platforms. For these cases, the interpulse distance $p$ is given by a new variable which we call the pulse distance

$$
D_{\text {pulse }}=p=V_{o} / P R F
$$

where $P R F=$ pulse repetition frequency $=1 / T$

and $\mathrm{T}=$ the interval between pulses, or interpulse period.

Thus synthetic aperture processing may be used for cases where the sonar/radar platform is sequentially moved (i.e. "stop-and-go"), or where the platform moves continually, and usually at a constant speed $\mathrm{V}_{0}$. 


\subsection{SAS Operation}

This section shows the features of focused, unfocused, and spotlight forms of synthetic aperture. A synthetic aperture radar or sonar focuses echoes (in one of the three following ways), and creates a map (or an image) of target reflectivity. The map is usually presented (for radar) on a gray scale, and resembles a high-altitude photograph. The reflectivity resulting from the processing may have either positive or negative values (depending on the phase), but since the magnitude is what matters, only the magnitude of the reflectivity is presented. SAR systems have the additional benefit (or complication) that they may transmit waves of different transverse polarization, but these features will not be addressed here. For acoustic propagation in a fluid, only longitudinal waves may exist, and so we confine our attention io these waves only. It is however possible to use polarized shear waves for acoustic propagation in solids, and this has been done in NDT applications. See the section on high-frequency SAS, in Chapter 1.2.2.

\subsubsection{Focused SAS}

Consider the geometry for a focused synthetic aperture, shown in Figure 2.10A. The $-3 \mathrm{~dB}$ mainlobe of the sonar beam, as transmiued from the sonar transducer (along-track length $D$ ), has the location of a postulated target at $x_{0}, y_{0}$ within its view for the platform travel distance $L$. Also shown is the shortest approach broadside distance $R_{0}$. We use the descriptor postulated because the actual number and location of targets is unknown, and it makes the analysis general for a target at any $x_{0}, y_{0}$ position. This is the geometry for the focused SAS case. The resolution $\rho$ in range (cross-track resolution) for the synthetic aperture, and for other radars and sonars, may be shown from consideration of the pulse time duration $\tau$, or the pulse bandwidth BW (in Hertz); this is shown in Appendix B, and repeated here as

$$
\begin{array}{ll}
\text { incoherent sonar: } & \rho_{\text {range }}=c \tau / 2 \\
& \\
\text { coherent sonar: } & \rho_{\text {range }}=c \begin{array}{c}
c \tau / 2 \\
\text { or } \\
c /(2 B W)
\end{array}
\end{array}
$$

The along-track resolution (azimuth resolution) may be found from the geometry of Figure 2.10A, after a slight modification, which will now be shown. 


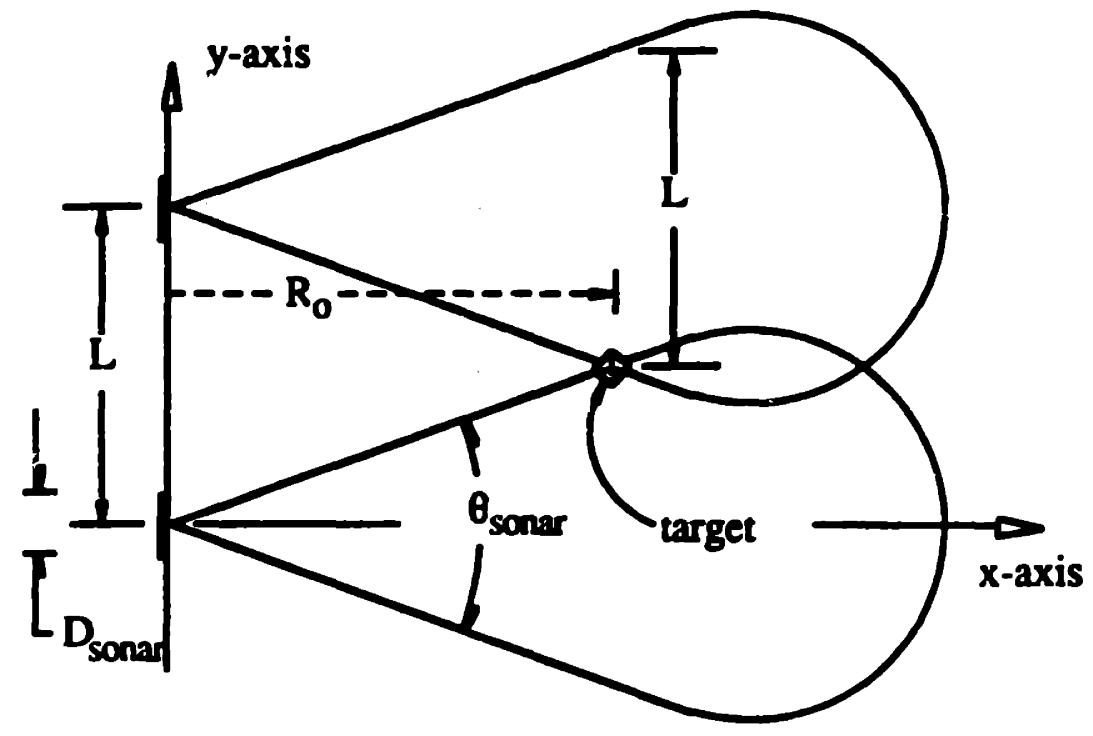

A. Geometry of First and Last Pulse over Synthesized Aperture Length L

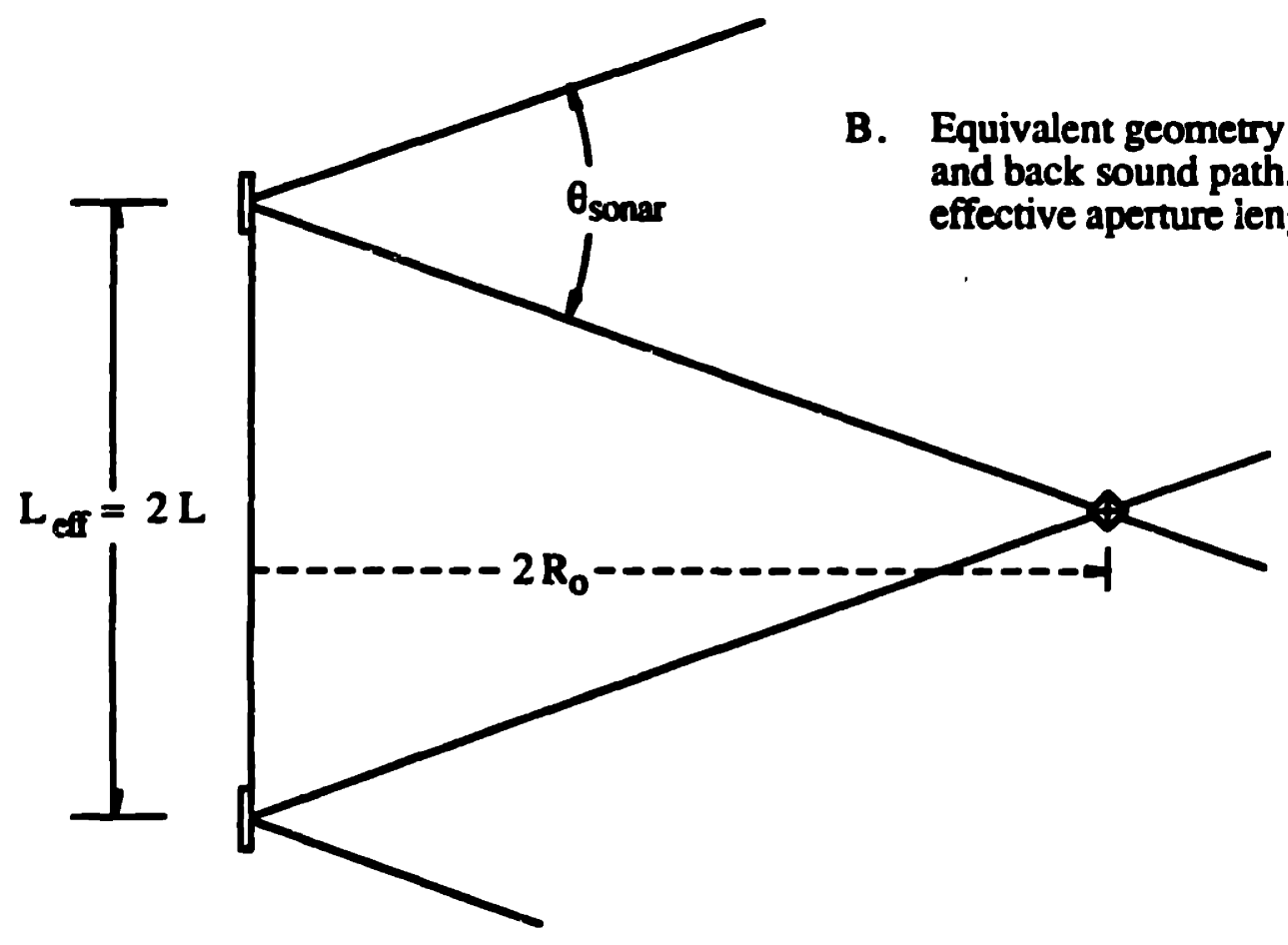

Figure 2.10 - Synthetic Aperture Lengths $\mathrm{L}$ and $\mathrm{L}$ eff 
If a sonar noisemaker (a tracking pinger for example) were located at $x_{0}, y_{0}$, and the sonar was used as a hydrophone, each sonar position $\mathbf{n}$ along the length $\mathrm{L}$ would receive the noisemaker sound after it traveled a distance $\mathbf{R}_{\mathbf{n}}$. This would be a one-way (receive-only) path. If the noisemaker were replaced by a sound reflriting target, and the sonar transmitted a pulse, and listened for echoes, also at sonar position $\mathbf{n}$ along length $\mathrm{L}$, the the path traveled for the echo would still be $R_{n}$, but the path for the sound pulse (i.e. the outback round trip path) would be $2 R_{n}$. We show the round trip geometry in Figure $2.10 \mathrm{~B}$. Note that this round trip geometry includes an effective length of the synthetic aperture $L_{e f f}$,

$$
\mathrm{L}_{\mathrm{eff}}=2 \mathrm{~L} \text {, }
$$

which preserves both the two-way path (out and back) and preserves the transmission/reception angle. The effective length $L_{\text {eff }}$ must then be remembered as not the true synthetic aperture length, but the apparent (effective) length due to the two-way nature embedded in the range migration. The concept of the effective length $L_{\text {eff }}$ is important for finding the along-track resolution. A useful alternate description of this feature of synthetic arrays may be found in Chapter 2 of Mensa's text [1981].

By the reasoning used for real apertures, we can now estimate the width of the sonar $-3 \mathrm{~dB}$ mainlobe for an array of length $L_{e f f}$. The $-3 \mathrm{~dB}$ main lobe width for an aperture of length $L$ is recalled from (2.2):

$$
\theta_{\text {mainlobe }} \approx \lambda / \mathrm{L}
$$

Taking the general form for the $-3 \mathrm{~dB}$ mainlobe width as $\lambda /$, we use the same form but now using $L_{\mathrm{eff}}$ for the synthetic aperture sonar

$$
\theta_{\mathrm{SAS}} \approx \lambda / \mathrm{L}_{\mathrm{eff}}
$$

We now make several substitutions of other, more useful variables:

$$
\begin{aligned}
& L_{\text {eff }}=2 L \\
& L \approx R_{0} \theta_{\text {sonar }}, \\
& \theta_{\text {sonar }} \approx \lambda / D_{\text {sonar }} .
\end{aligned}
$$


which gives the result

$$
\theta_{\text {SAS }} \approx \lambda / L_{\text {eff }}=D /\left(2 R_{0}\right)
$$

The along-track resolution $p_{\text {along-track }}$ for a target at range $R_{0}$ is approximately the range $R_{0}$ multiplied by the $-3 \mathrm{~dB}$ mainlobe angular width $\theta_{S A S}$, or

$$
\text { Palong-track }=R_{0} D /\left(2 R_{0}\right)=D / 2 \text {. }
$$

This is a result that is frequently cited as the reason why (focused) synthetic aperture radar gives phenomenal airborne and spacebome images -- because the along-track resolution (azimuthal resolution) is: independent of both range and frequency, and is actually smaller than the size (along-track length) of the transmitter-receiver. This remarkable result is a consequence of the similar triangles argument shown previously. Targets at great ranges require the sonar platform to travel large distances $L$ in order that the target be in sonar view from one side of the $-3 \mathrm{~dB}$ mainlobe to the other. Likewise, targets at short range require the sonar to travel only a short distance for the target to sweep across the sonar $-3 \mathrm{~dB}$ mainlobe. However, for both cases, $R_{\boldsymbol{O}} / L$ is constant because $\theta_{\text {sonar }}$ is constant, and so the resolution in the alongtrack direction is also constant and equals $\mathrm{D} / 2$.

Two additional comments must be made at this point. The first is that synthetic apertures do not really form beams, but rather they focus the ensemble of received echoes everywhere in the image space to find targets. In fact, to form a complete image a synthetic aperture focuses the data at all points that were in the view of the sonar (or radar), because the area being imaged may be filled with many targets at equally many unknown locations. Focusing at all points (or at least at all points spaced by resolution-size steps) creates a 2-dimensional (2-D) map of target reflectivity, which we then call an image. Focusing itself can be described as a beamforming step which takes the beamwidth (which only exists, by the definitions of Appendix A, in the far field) and brings it into the near field. The math shown above inherently assumes that focused processing occurs which allows the far field beamwidth to be brought into the near field (about $L_{\text {eff }} 2 / \lambda$ from broadside along the $x$-axis).

The second comment is that synthetic apertures, especially radars, are often approached from a Doppler viewpoint. The explanation of sy.thetic aperture given in this thesis has not mentioned Doppler shifts until now, and instead showed the principle of synthetic apertures from geometry considerations. It was also shown that synthetic apertures may be made from stop-and-go measurements, as in certain types of ground penetrating radar and in NDT, where the platform is stationary during transmission and reception and 
is moved after all echoes are received. In this case, there is no Doppler shift present in any of the signal returns because the transmitter and the target area were both temporarily stationary. The key then is that as the transceiver is sequentially moved, thereby changing the geometry, there is an accompanying change in the number of wavelengths (round trip) between transceiver and any single target, which means that the phase over the round trip path changes. It could be said that the phase change is actually a phase migration, which exactly corresponds to the range migration of a target. In contrast for the case of a transceiver platform which moves at a constant speed rather than being used in a stop-and-go fashion, there is stull a phase change, or phase migration, because the geometry is no different than would be found in the stop-andgo case. The difference is that the platform moves at a rate, and therefore the geometry and hence the phase must also change with the same rate. A rate of change in phase is frequency, and since the rate of phase change varies with platform position, then the frequency must also change. This is the Doppler shift (see Appendix D). So the moving transceiver detects Doppler shifts in the received pulses. Hence the longestablished Doppler viewpoint found in many SAR papers is correct, but it may also be reconciled purely by geometrical means. A similar, more mathematical explanation of this may be found in Munson $\mathrm{Jr}$. and Visentin [1989].

\subsubsection{Unfocused SAS}

The focused synthetic aperture method described above works on the full ensemble of echoes from a postulated target at $x_{0}, y_{0}$, as the target at $x_{0}, y_{0}$ sweeps across the entire sonar $-3 \mathrm{~dB}$ mainlobe during the sonar transit across the distance $L$. The focused nature of the processing has to do with the fact that the echo for a target at $x_{0}, y_{0}$ is located at a certain time (or in a time interval) in each time record. Equation 2.14 may be rewritten to describe the hyperbolic arc range migration $R_{n}$ as a function of $y$,

$$
R_{n}=\sqrt{R_{0}^{2}+\left(y_{n}-y_{0}\right)^{2}} \text {, }
$$

where $\mathbf{R}_{\mathbf{0}}=\mathbf{x}_{\mathbf{0}}$.

We can let $y_{0}$ equal zero for the sake of simplicity, and then expand (2.25) in a Taylor series:

$$
R_{n}=R_{0}+\frac{1}{2 R_{0}} y^{2}-\frac{1}{8 R_{o}^{3}} y^{4} \ldots .
$$


This series is often approximated by

$$
R_{n}=\frac{1}{2 R_{0}} y^{2}+R_{0}
$$

Equation 2.27 is a parabolic or quadratic approximation of the hyperbolic range migration arc. Focused processing must calculate where this arc intercepts each record in the ensemble to determine where the echo for a target at $x_{0}, y_{0}$ should be (if there were really a target there). The unfocused synthetic aperture does not make this calculation, so it is a suboptimal method. It reduces the number of processing steps while, at the same time, it degrades the along-track resolution. Like the focused case, the unfocused case may be best explained by geometry.

Consider Figure 2.11. Three extreme sonar positions are shown relative to a single point target located at $x_{0}, y_{0}$ (broadside range $R_{0}$ ). Since the assumption in unfocused synthetic aperture processing is that we essentially ignore the range migration, then we must limit the length of the synthetic aperture by the allowed change in sonar-to-target range by $\lambda / 4$. The unfocused synthetic aperture process may also be regarded as focusing at an infinite range ('focusing at infinity'). When this occurs, for practical purposes, the range migration hyperbolic arc becomes a straight line.

Recall that the propagation path is two-way (out and back), so a one-way limit is set at $\lambda / 8$ (customary in SAR literature) which gives $\lambda / 4$ for the round trip path. We choose $\lambda / 4$ because two identical sinusoidal waves (the received echo and its stored transmit replica for example) nay be phaseshifted by as much as $\lambda / 4$ and still give a positive value for a cross correlation (see Appendix B for explanation).

Figure 2.11 shows the broadside (closest approach) distance $\mathbf{R}_{\mathbf{0}}$, as well as $\mathbf{R}_{\text {first }}$ and $\mathbf{R}_{\text {last }}$ (the range for the first and last pulses along the unfocused length $L$ ). By symmetry, $R_{\text {first }}$ and $R_{\text {last }}$ are approximately the same length, and we limit the length $L$, by the restriction that

$$
R_{n} \leq R_{0}+\lambda / 8
$$

And by the geometry of Fig. 2.11,

$$
R_{n}^{2}=\left(\frac{L}{2}\right)^{2}+R_{0}^{2}
$$




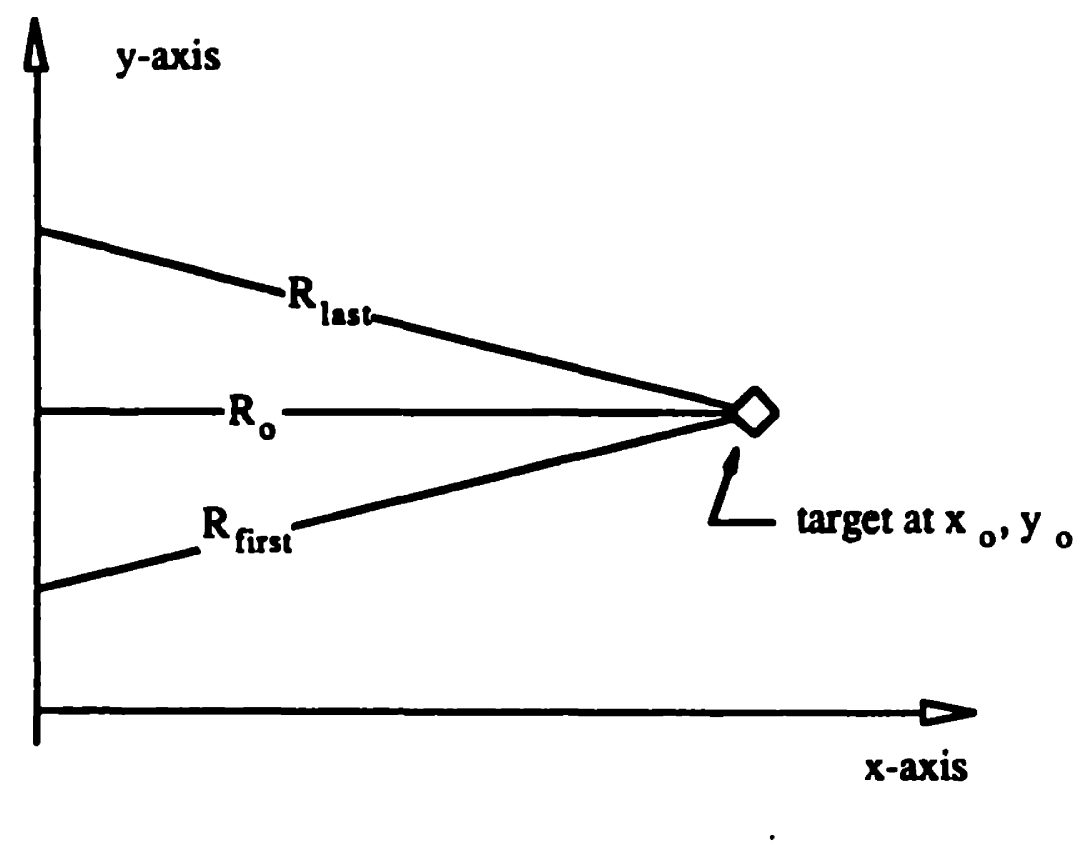

Figure 2.11 - Geometry for Unfocused SAS 
Since $R_{\text {first }}$ equals $R_{\text {last }}$ under ideal circumstances, and both of these are the maximum value of $R_{n}$, then we substitute (2.28) into (2.29) and using an equality, and solve for the maximum value of $L$ :

$$
\mathrm{L}^{2}=\mathrm{R}_{0} \lambda+\frac{\lambda^{2}}{16}
$$

We assume that $R_{0} \lambda$ is substantially greater than $\lambda^{2} / 16$, and so

$$
L \cong \sqrt{R_{0} \lambda}
$$

From the previous section on focused SAS, we observed that the effective length of a synthetic aperture is twice as long as the geometric length $\mathrm{L}$, or that $\mathrm{L}_{\text {effective }}=2 \mathrm{~L}$, due to the two-way out-and-back path which the sonar waves travel. This also occurs here in the unfocused case for exactly the same reasons. Thus,

$$
L_{\text {effective }}=2 \mathrm{~L}=2 \sqrt{R_{0} \lambda} \text {. }
$$

From the previous sections on both real apertures and on focused apertures, we know that the along-track (or azimuthal) resolution for an aperture for small $\theta$ is of the form

$$
\text { resolution }=\mathbf{R} \boldsymbol{\theta}=\mathbf{R} \frac{\boldsymbol{\lambda}}{\mathrm{L}} \text {, }
$$

so for the unfocused case, the along-track resolution is given by

$$
\rho_{\text {along-crack }}=\frac{\sqrt{R_{0} \lambda}}{2}=L / 2 \text {. }
$$




\subsubsection{Spotlight SAS}

The spotlight form of synthetic aperture sonar (SAS) deliberately steers the real mainlobe of the sonar at the area of interest, and continues to do so for the duration of the sonar transit. Since the sonar beam is continually trained upon a particular spot, it was named after the theater spotlight it resembles. Steering a sonar in this fashion may be done in several ways. For a single transducer sonar, the sonar element must be mechanically pointed in the proper direction, during the "fly-by" of the sonar. If the sonar is comprised of a small, real array, the elements of the array may themselves be time- or phase-delayed to affect the steering of the mainlobe. Both these methods assume that the sonar platform flight path is straight. An alternate method is to fly the platform along a circular path with the target area located at the circle center; this would always keep the target area at broadside of the sonar, which both focuses and create a spotlight aperture. This would also simplify the processing because the target area is always at the same range from the sonar, so the hyperbolic range migration arc (e.g., as shown in Fig. 2.7) becomes a straight line in the $y-R_{n}$ space. This technique was used by Moshfeghi [1986].

Since the mainlobe of the sonar is steered by one of these methods, the target area never leaves the view of the sonar and this implies that the length of the synthetic aperture may be made indefinitely long; an indefinitely long aperture means vanishing small (or high) along-track resolution by the notion that resolution for any aperture, real or synthetic, is inversely proportional to aperture length. An example will show how the along-track resolution may be determined, and like the previous focused and unfocused SAS cases, we always assume coherent processing, and we assume that the range resolution is determined by pulse compression (see Appendix B).

R.S. Powers [see Synthetic Aperture Radar. Technology and Applications, Vol. I, p. 4-14, 1989] presents without proof the azimuth resolution for a spoulight SAR:

$$
\rho_{\text {along-track }}=\frac{\lambda}{4 \sin \left(\frac{\Delta \theta}{2}\right)} \approx \frac{\lambda}{2 \Delta \theta}
$$

This result may be explained by the following geometrical argument. A platform begins a fly-by on a target at $x_{0}, y_{0}$ as shown in Figure 2.12, where $x_{0}=R_{0}$ (the broadside range). 


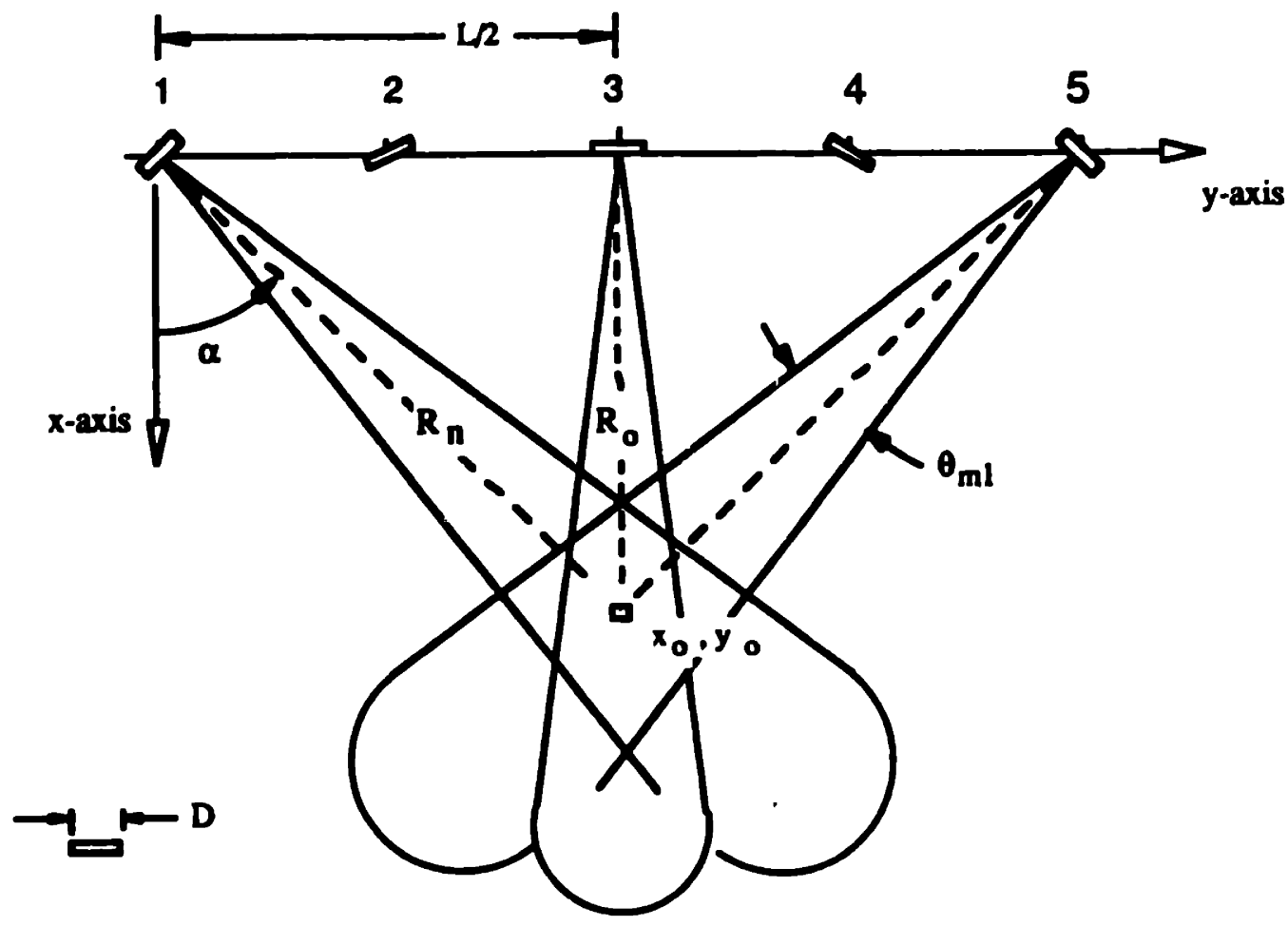

Figure 2.12 - Geomery for Spotlight Synthetic Aperture 
Five platform positions are shown in this Figure, and labeled as stations 1 through 5 . We assume the platform begins transmitting (and recording data) at 1 and finishes at 5 . Note that the transceiver is always pointed towards the target area at $x_{0}, y_{0}$. At station 1 the angle from broadside to the target is $\alpha$. Since stations 1 and 5 are respectively the beginning and end of the data collection, then the distance from 1 to 5 is the length of the synthicic aperture, which we call $L$. We now find $L$ in terms of $\alpha$ and $R$ :

$$
\begin{aligned}
& \sin (\alpha)=\frac{L D}{R_{n}}, \\
& L=2 R_{n} \sin (\alpha)
\end{aligned}
$$

We recall by a previous argument that $2 \mathrm{~L}=\mathrm{L}_{\text {eff }}$ due to the two-way, out-and-back propagation path. We make this substitution:

$$
L_{\text {eff }}=4 R_{n} \sin (\alpha)
$$

Let's also make a change of notation where

$$
2 \alpha=\Delta \theta
$$

or

$$
\alpha=\frac{\Delta \theta}{2} \text {. }
$$

The new variable $\Delta \theta=2 \alpha$ is the total angular swath of the sonar during its travel over length $L$, while being trained upon $x_{0}, y_{0}$, and uses the notation of Powers. Making this substitution for the angle:

$$
L_{\text {eff }}=4 R_{n} \sin \left(\frac{\Delta \theta}{2}\right)
$$

The along-track resolution is recalled from earlier sections, and given by the familiar formula

$$
\rho_{\text {along-track }}=\frac{\lambda}{L} R \text {, }
$$


and so we substitute in the values for $L=L_{e f f}$, and $R=R_{0}$ :

$$
\rho_{\text {along-track }}=\frac{\lambda}{4 R_{n} \sin \left(\frac{\Delta \theta}{2}\right)} R_{0}
$$

If we now make the assumptions that $R_{0} \approx R_{n}$, and that $\sin (\Delta \theta / 2) \approx(\Delta \theta) / 2$, then we find that

$$
\rho_{\text {along-track }}=\frac{\lambda}{2 \Delta \theta} \text {. }
$$

which is the result given by Powers. This result for along-track resolution contrasts with the results for real apertures and for focused synthetic apertures, which are summarized in Table 2.1. For more details of spotlight synthetic apertures, see R.S. Powers, Synthetic Aperture Radar. Technology and Applications, Vol. I. Chapter 4, [1989].

Table 2.1 - Along-Track Resolution Compared

along-track resolution*

$\begin{array}{lc}\text { real aperure } & \frac{R_{0} \lambda}{D} \\ \text { synthetic apertures: } & \frac{\sqrt{R_{0} \lambda}}{2} \\ \text { unfocused } & \frac{D}{2} \\ \text { focused } & \frac{\lambda}{2 \Delta \theta} \\ \text { spotlight } & 2\end{array}$

- based on -3 dB sonar mainlobe width. 


\subsubsection{Target Reflectivity for Changing Sonar Positions}

Nothing has been said, thus far, concerning the reflecting properties of the target at $x_{0}, y_{0}$. Real targets are not point targets (having vanishing small size, and uniform target reflection strength, for any angle of sound incidence). Real targets have reflection characteristics that depend on both geometry and composition, and are, therefore, much more difficult to analyze.

Since a region under the scrutiny of a synthetic array (whether radar or sonar) is probably unknown, we must assume that targets could be located at any position, and we must also assume that the targets are small enough to have a reflectivity that is independent of incident angle. These assumptions are made frequently in radar and sonar, when the number, location, size, geometry and composition of targets within an area is unknown. This introduces an artifact in the image which is created from an area, because, we are in-effect, averaging the echo from any one target location over a large number of pulses, which were made from a moving sonar (or radar) platform, and therefore for a large number of incident angles.

\subsection{Sampling Requirements and Sidelobe Limits}

An aperture, whether it is real or synthetic, that has been discretized from a continuous length (or area for a 2-D array) into pieces, has definite sampling requirements which must be satisfied to avoid aliasing. For the array point of view, the aliasing comes in the form of aliasing lobes, which are sonar beam lobes that have nearly the same magnitude (strength) as the mainlobe. For the synthetic array, the aliases come in the form of alias (false) targets. This section discusses these sampling requirements particularly as applied to the synthetic array, and also discusses the sidelobes for synthetic arrays. For a good general discussion on sampling, see sections 2.4 and 2.5 in Woodward [1953].

\subsubsection{Sampling Requirements}

We identify the sampling requirements of a synthetic array by looking at the geometry of the array along the $y$-axis (the direction of platform motion), and by looking at the "beam pattern" space (or the wavenumber space) which is the Fourier transform of the aperture along the $y$-axis. Consider Figure 2.13, which has eight parts. The four parts on the left, labeled A, B, C and D, are along the $y$-axis, and their counterparts (on the right-side of the page, $A^{\prime}, B^{\prime}, C^{\prime}$, and $D^{\prime}$ ) are their respective Fourier transforms. Appendix A shows the formulation of the beam pattern for an aperture, and how it is mathcinituically a 
Fourier transform $\rightarrow$

$\leftarrow$ inverse transform

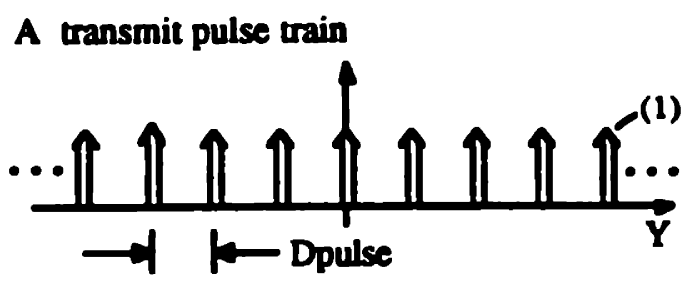

$\mathbf{x}$

B aperture length $\mathrm{L}$

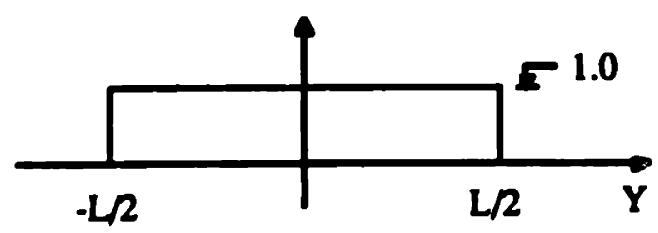

C sonar length $D$, transmit

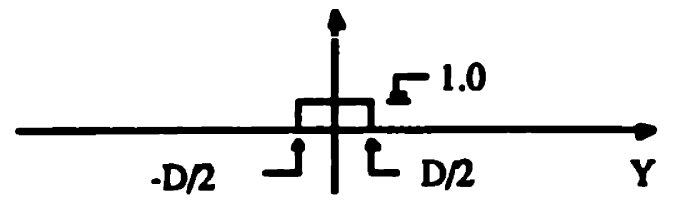

D sonar length $D$, receive

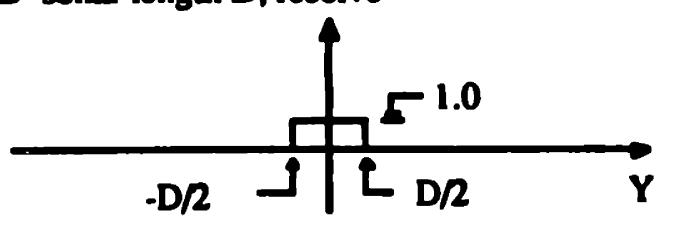

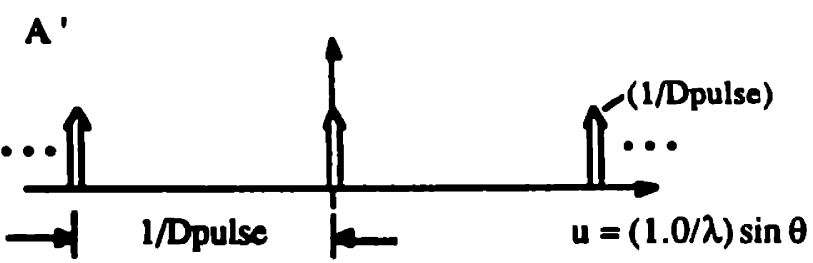

*

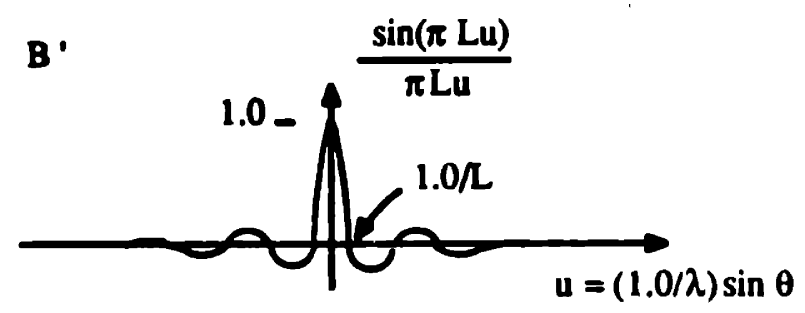

$\mathbf{x}$

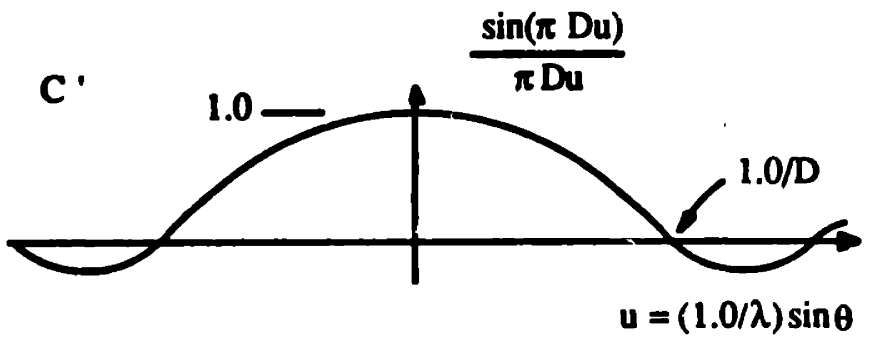

$\mathbf{x}$

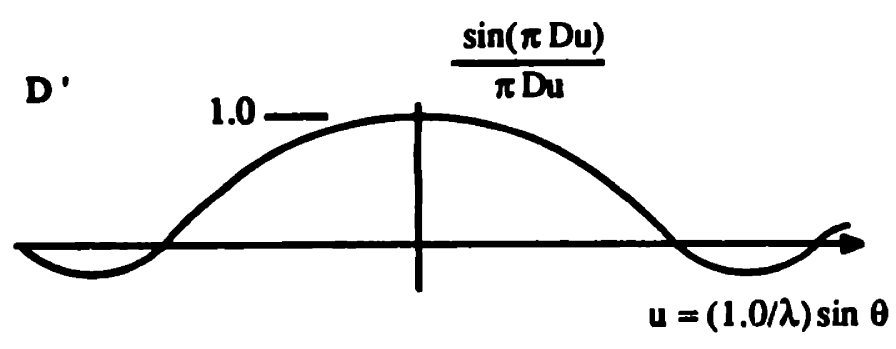

Figure 2.13 - Transform Pair: Geometry and Wavenumber Spectra 
Fourier transform so long as the range $\mathrm{r}$ is in the far-field of the aperture. We skip the lengthy steps of the Appendix here, and simply say that the Fourier transform of the aperture geometry gives the beam pattern (or wavenumber spectra), referring to the Appendix for the proof. Furthermore, since we use either the focused or unfocused processing and Ireat the time series for each pulse as the input to an array element (e.g. Fig 2.9 using a time-after-pulse time scale), we have effectively moved the far-field beam pattern into the near-field, and recognize that the transform domain (also called the wavenumber domain) is really an azimuthal image for a single target centered at $x=R_{0}$ (the location of the focus).

Returning now to Figure 2.13 and all future transform pairs in this thesis, we note that the " $x$ " symbol denotes multiplication, and that the "*" symbol denotes convolution (see Woodward [1953] or Siebert [1986] for details of the convolution). These symbols are used to show either the multiplication or convolution of the figures located, respectively, above and below the symbol.

Item $A$ in Fig. 2.13 is a train (or "picket fence") of Dirac delta functions that are regularly spaced at $D_{\text {pulse, }}$ where $D_{\text {pulse }}$ is the interpulse distance given by (2.16), and recalled here:

$$
D_{\text {pulse }}=\frac{V_{0}}{P R F}
$$

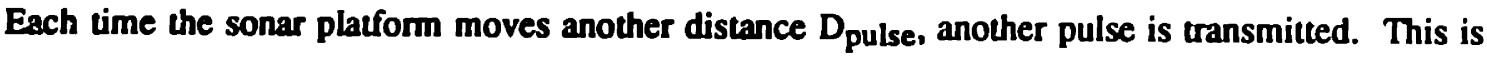
represented by a delta function for each sonar pulse. For now, we ignore the form of the pulse (the duration and frequency content) and instead concentrate on the locations of these pulse delta functions along the $y$ axis. Between each pulse, the sonar platform moves at speed $V_{0}$ and continuously records echoes. Note that item A shows pulses that are intentionally close together. This feature is important for sampling purposes, and will be recalled shortly. Item B shows the rectangular aperture of length $\mathrm{L}$, which is a distance over which a synthetic aperture could be formed, but for our purposes the distance $L$ really represents the effective distance $L_{\text {eff }}\left(L_{\text {eff }}=2 L\right.$ from Fig. 2.10$)$. We multiply item $A$ by item $B$, which gives us a pulse train (spaced at $D_{\text {pulse) }}$ under a rectangular envelope. This result is then twice convolved by the rectangle aperture for the sonar (having length $D$ ), once for the transmit and once for the receive.

Some minor approximations have been made in the y-location of $C$ and $D$ in Fig. 2.13, and this deserves some comment before proceeding. C represents the transmit sonar aperture which is conveniently centered at the y-origin. D, the receive sonar aperture, is also centered on the origin at the $y$-axis. This is technically incorrect unless we are using a stop-and-go SAS (or SAR) system like the ground penetrating radar mentioned in section 2.1.2. For a SAS or SAR system which has a platform travelling at speed $V_{0}$, 
the receive position along the $y$-axis must be displaced by the platform velocity multiplied by the time-offlight for a target echo. Furthermore, since the transmit signal has a finite duration in time, then both the transmit and receive location occur along the $y$-axis as functions of time. These features are not shown in Figure 2.13 for the sake of a simple explanation; however, these features are incorporated in the processing which will be described in a later chapter.

Turning now to the right-hand side of Figure 2.13, we note the transform counterparts: $A^{\prime}$ is a delta function train with wide separation, $B^{\prime}$ is a sinc function (the transform of a rectangle, or box-car) which is very narrow (because $L$ was long), and similarly $C^{\prime}$ and $D^{\prime}$ are sinc functions (which are very wide because length $D$ was small). $A^{\prime}$ is convolved with $B^{\prime}$, and the result is then multiplied by $C^{\prime}$ and $D^{\prime}$. Nore that the multiplication on one side of the page is the counterpart of convolution on the other side of the page.

Since we are ultimately interested in images resulting from the use of a synthetic array, only the transform side of the page will be carried out. The results are shown in Figure 2.14 in three parts. Item $E^{\prime}$ is the result of convolving $A^{\prime}$ and $B^{\prime}$ of Figure 2.13, and this is a train of sinc functions which are spaced at $1 / p=1 / D_{\text {pulse, }}$ or spaced at PRF/V $V_{0}$. These sinc functions occur at, what has been called in the radar literature, the "PRF lines". Item $F^{\prime}$ is the result of multiplying. $C^{\prime}$ by $D^{\prime}$, and is a sinc ${ }^{2}$ function. $G$ ' is the final image result, and is the multiplication of $E^{\prime}$ by $F^{\prime}$. Several features must now be discussed. With the exception of the center lobe, the regular train of sinc functions in item $\mathrm{E}^{\prime}$ are the alias lobes of a discrete array of length $L$. The usual way to avoid these lobes in a real aperture is to ensure that the spacing $p$ (along the $y$-axis) is less than $\lambda / 2$. In the transform domain, this causes the lobes nearest to the center lobe to be located at an angle $\theta>\pi / 2$. Since we assume the sonar resides in a rigid flat baffle, then the alias lotes are forced into the baffle plane and therefore are not physically real.

For a small moving sonar which creates a synthetic aperture, we could make similar demands on how frequenuly we sample, say as frequently as $\lambda / 2$ for the interpulse distance $p=D_{\text {pulse }}$, but this is an unnecessary burden and there is a better way to avoid the aliasing lobes. Inspection of items $E^{\prime}$ and $F^{\prime}$ in Figure 2.14 shows that if we could place the aliasing lobes of $E^{\prime}$ and the nulls of $F^{\prime}$ at the same position $u$, then the alias lobes would be zeroed upon multiplying $E^{\prime}$ and $F^{\prime}$, and a single (image) lobe will result, which is exactly how $G^{\prime}$ appears. We will now show this in two steps: first, we will sct the first null position, at $u=1 / D$, for item $F^{\prime}$ to be equal to the position of the first alias lobe, at $u=1 / D_{\text {pulse, }}$ which gives us an almost correct answer; second, we will correct the defect by recognizing the sampling needed to avoid alias overlap. 


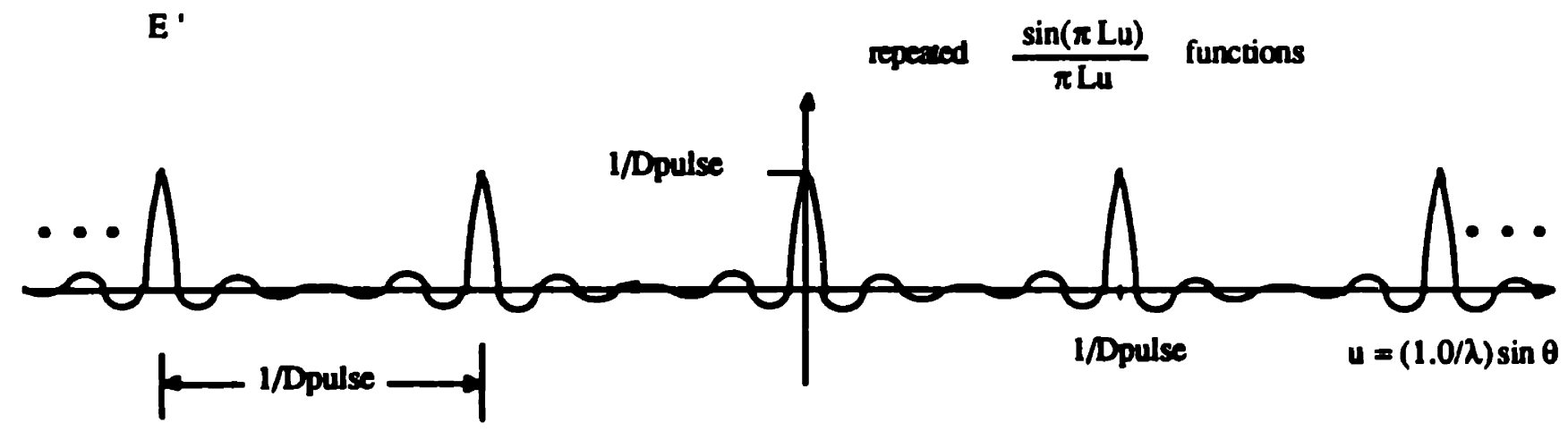

$\mathbf{X}$
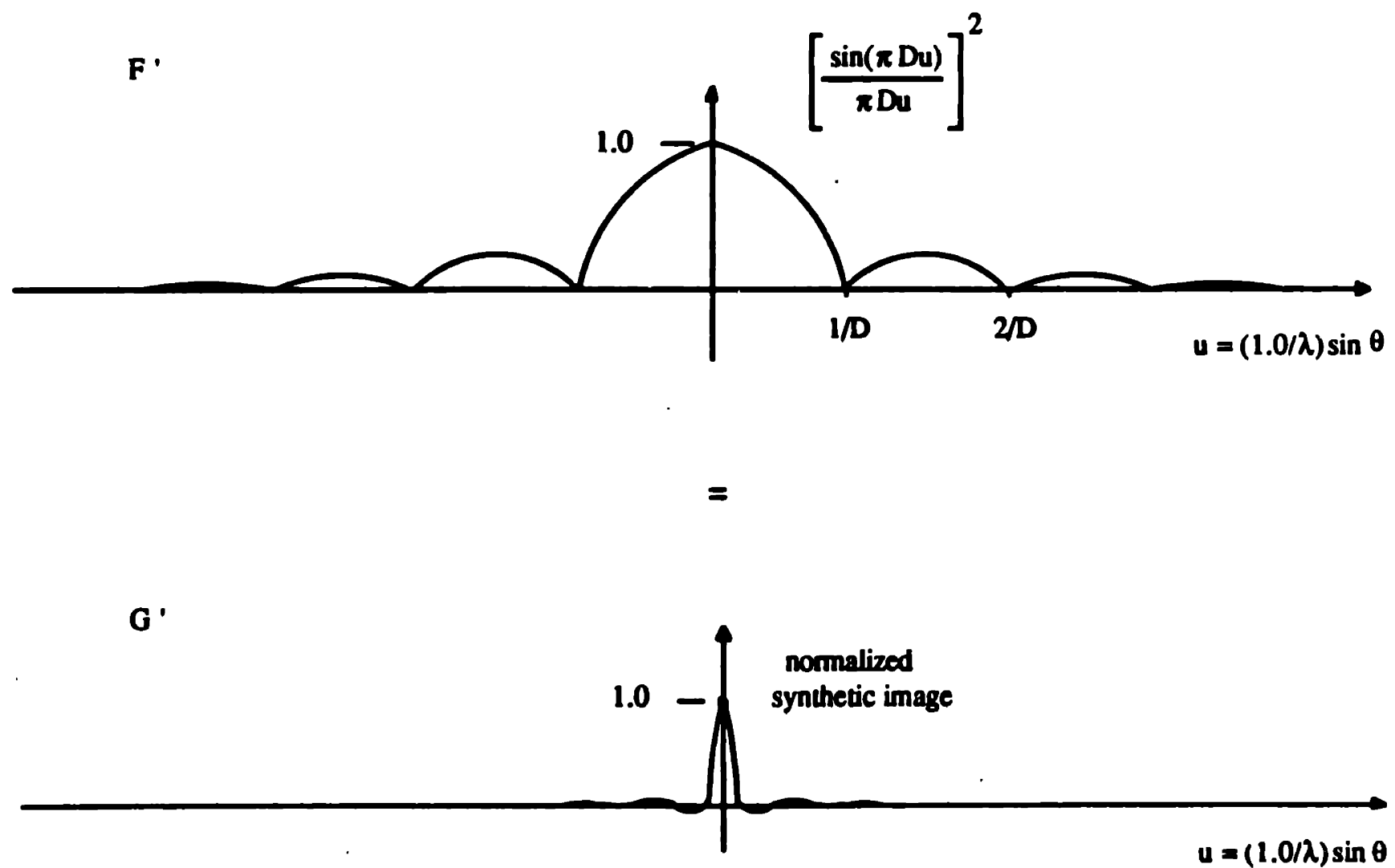

Figure 2.14 - Transform Result of Fig. 2.13 
Set the first positive null of $F^{\prime}$ to be equal to the first positive alias lobe in $E^{\prime}$, in Figure 2.14:

$$
u=\frac{1}{D}=\frac{1}{D_{\text {pulse }}}
$$

which gives the result that

$$
D=D_{\text {pulse }}
$$

Equation 2.44 almost gives the ccrrect answer. What was overlooked was that we wish to exclude the influence of the first alias lobe (located at $u=1 / D_{\text {pulse}}$ ) from the (wavenumber) band from zero to $1 / D$, because the region from -1/D to 1/D is the null-to-null angular field of view for the length $D$ sonar. This means that we need to sample $u$ in item $E^{\prime}$ at 'east twice as fast as we sample $u$ in $F^{\prime}$. This is a restatement of the sampling criteria (often called the Nyquist rate, see Sieber [1986]), which states that you always need to sample at least twice as fast (in the frequency domain, or in the wavenumber domain) as the highest point of interest in the band. For our purposes, we are concerned with a large band in $u$, but we are specifically concerned with nulling the alias lobes introduced by the periodic pulsing. To nullify the first alias lobe (located at $u=1 / D_{\text {pulse}}$ ) and satisfy the Nyquist criteria, we need to sample twice as fast as the first null position (at $u=1 / D$ ), or as fast as 2/D. Restated, we need to place the first alias lobe exactly at $u=2 / \mathrm{D}$, to null the lobe, or we need to place the lobe beyond (greater than) $2 / \mathrm{D}$ to minimize it:

$$
\frac{1}{D_{\text {pulse }}} \geq \frac{2}{D} \text {. }
$$

Solving (2.45) for $D_{\text {pulse: }}$

$$
D_{\text {pulse }} \leq \frac{D}{2} \text {. }
$$

Equation 2.46 is the correct answer, and it means that a pulse must be transmitted no less than each time the sonar platform moves a distance equal to half the sonar aperture length $D$. So the distance necessary to satisfy spatial sampling (along the y-axis) is less-than, or equal to, D/2. We also recognize that both the aliasing lobes of $E^{\prime}$ and the nulls of $F^{\prime}$ are regularly spaced. So, if we succeed in nulling the first (positive u-axis) alias lobe, we shall succeed in nulling all lobes except the center lobe. Likewise, if we oversample beyond the Nyquist rate, we will not null the alias lobes, but they will be supprin...1 by the 
sidelobes of sonar beam pattern. There are several ways of showing the result of (2.46) and the reader is directed to Tomiyasu [1978] for the other methods.

The concept of nulling the alias lobes is one that has been shown in much of the synthetic aperture literature, for both radar and sonar. There are limitations, however, to how well the null of the sonar beampattem will erase the alias lobe. The origin of these limitations will be shown in section 2.3.2.

At the beginning of this section (2.3.1) we temporarily ignored the form of the transmit pulse and confined our interest in a geometry-wavenumber transform approach to identify sampling requirements. Let's now introduce a time-frequency approach to show the same sampling requirements. Consider Figure 2.14' which shows Fourier transform pairs: time functions $A$ through $D$ on the left, and their respective frequency functions $A^{\prime}$ through $D^{\prime}$ on the right. Item $A$ shows a sinusoidal wave, which is multiplied by the rectangular 'box-car' B, which gives us the transmit pulse (the sine wave is chosen for ease of illustration, but more complex waveforms may also be used). Since we wish to transmit the signal every $T$ seconds (the period), then we convolve the A-B produc! with $C$. The result is shown in D as a train of sinusoidal pulses beneath box-car windows at every $T$-seconds spacing.

Tuming now to the frequency spectra, we observe $A^{\prime}$ as a narrowband signal (as illustrated by symmetric delta functions at $+/-f_{0}$, which is convolved with the sinc function $B^{\prime}$. The result of $A^{\prime} * B^{\prime}$ is then multiplied by $C^{\prime}$, a train of delta functions spaced at PRF intervals (these are also PRF lines, only on frequency space). The end result is shown by a compressed scale view $D^{\prime}$ which shows two sinc-function envelopes centered at $+/-f_{0}$, and enclosing the PRF lines. Examination of $D^{\prime}$ allows a second look at the sampling requirements of a SAS or SAR.

Since SAS and SAR platforms collect data while in motion, there will be Doppler shifts in the target echoes. This will cause the PRF lines in D' 10 shift from maximum up-Doppler to maximum downDoppler. Assuming that the radar or sonar boresight is directed at broadside then the total Doppler shift (in Hertz) is given by

$$
\text { Doppler shift }=\frac{2 V_{0} \sin \left(\frac{\theta}{2}\right)}{c} f_{0}=\frac{2 V_{0} \sin \left(\frac{\theta}{2}\right)}{\lambda} \text {, }
$$

where the $\theta / 2$ is the $-3 \mathrm{~dB}$ half width of the real aperture beam. 
Time

Fourier transform $\rightarrow$

Frequency

$\leftarrow$ inverse transform

A

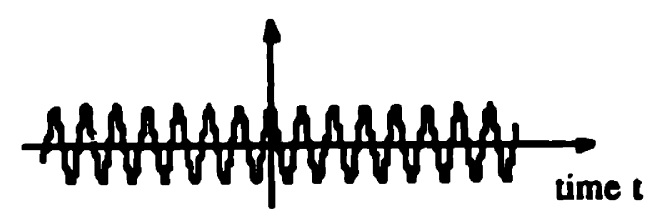

$\mathbf{x}$

B

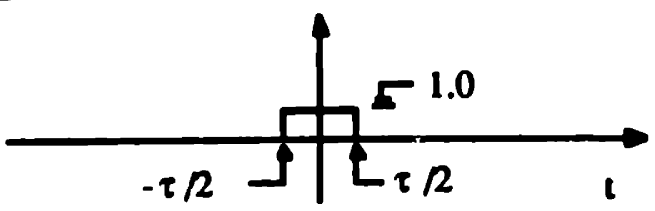

*

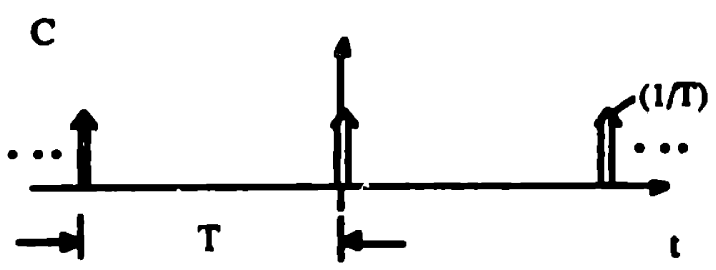

$$
=
$$

D

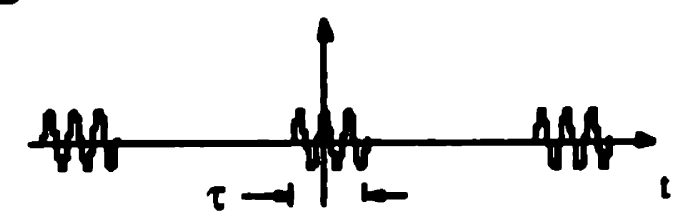

A

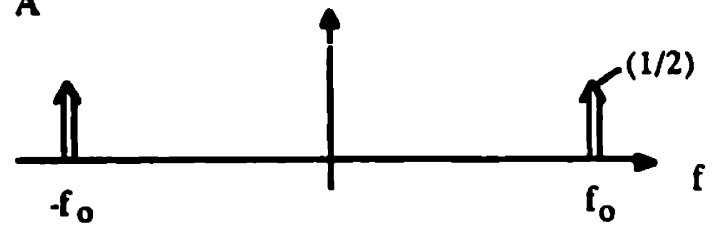

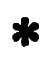

B '

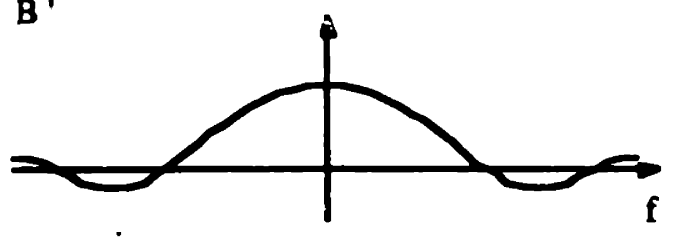

$\mathbf{x}$

$C^{\prime}$

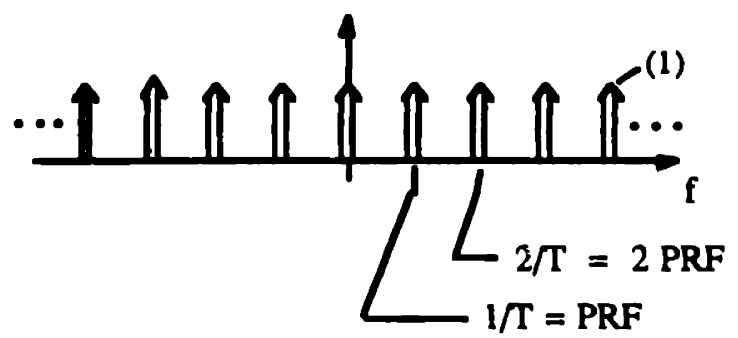

$$
=
$$

$D^{\prime}$

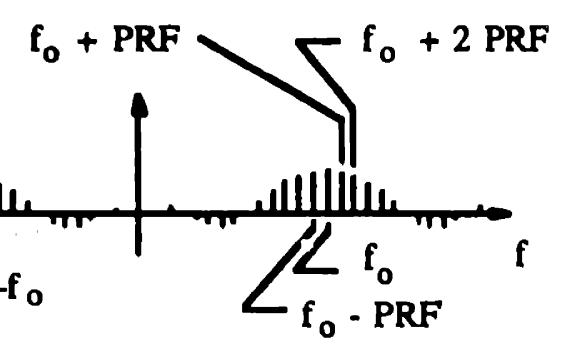

Figure 2.14' - Transform Pair: Transmit Signal and Frequency Spectra 
If we introduce the small angle approximation for $\theta$, and substitute $\theta=\lambda / \mathrm{D}$, we find

$$
\text { Doppler shift }=\frac{V_{0}}{D}
$$

Observation of D' in Figure 2.14' suggests that we want to ensure that any Doppler shift be less than one half of the PRF-to-PRF line distanc; this means we require that

$$
\text { Doppler shift } \leq \frac{\mathrm{PRE}}{2} \text {. }
$$

Recalling that $P R F=V_{C} / D_{\text {pulse, }}$ and substituting (2.48) into (2.49) we find that

$$
D_{\text {pulse }}<\frac{\mathrm{D}}{2}
$$

which is the result observed in (2.46).

\subsubsection{Limitations to Crushing Alias Lobes with the Sonar Beampattern Null}

This section will be explained from the grassroots of the image processing in order to provide physical, geometrical insight into the nature of aliases. This contrasts with the wavenumber approach used in the previous section, and will give us a framework from which we may describe how aliases are 'crushed' by the sonar beampattern null, and what the limits are in accomplishing this feat.

Consider the 2-D Figure 2.15, where we have a single small target located at $x_{0}, y_{0}$ on a perfectly smooth reflecting surface, and a radar or sonar platform which makes a 'flyby' at the $y$-axis along-track speed $V_{0}$. The sonar transmits pulses at the rate $P R F$ (in $\mathrm{Hz}$ ), and this gives a $D_{\text {pulse }}=V_{0} / P R F$. We also note a second geometric feature on the surface, indicated by the box labeled alias at $x_{0}, y_{0}+\Delta y$. 


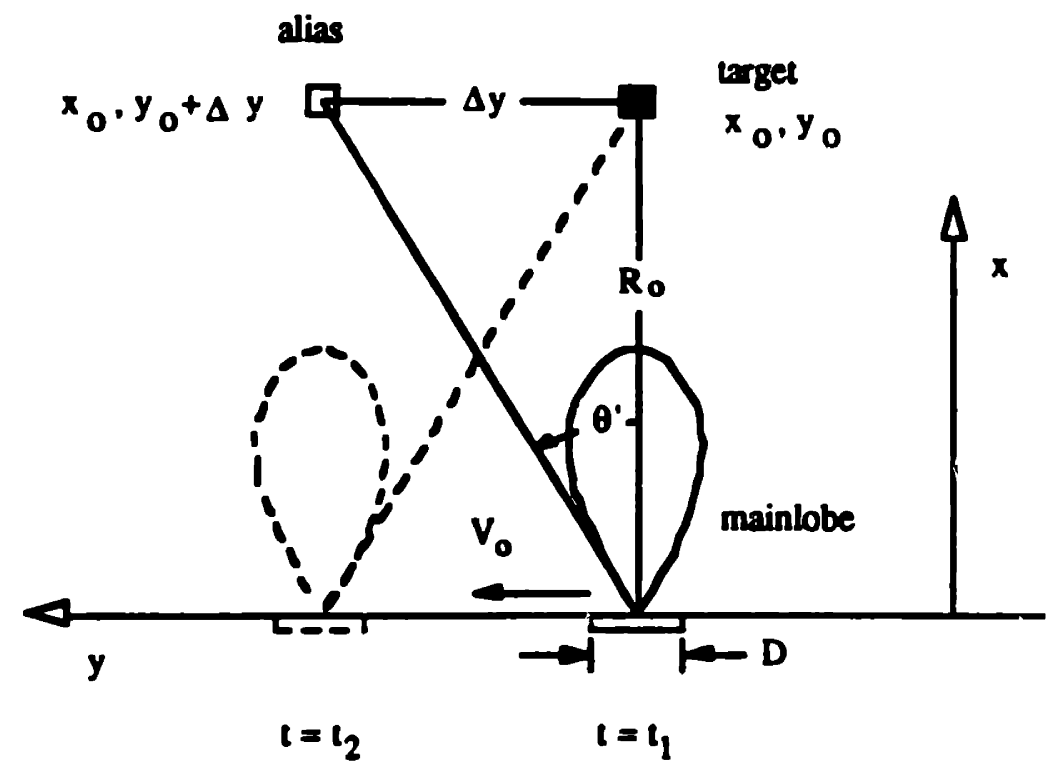

Figure 2.15 - Geometry for Target, Alias Target Image and Null of Sonar Beampattern

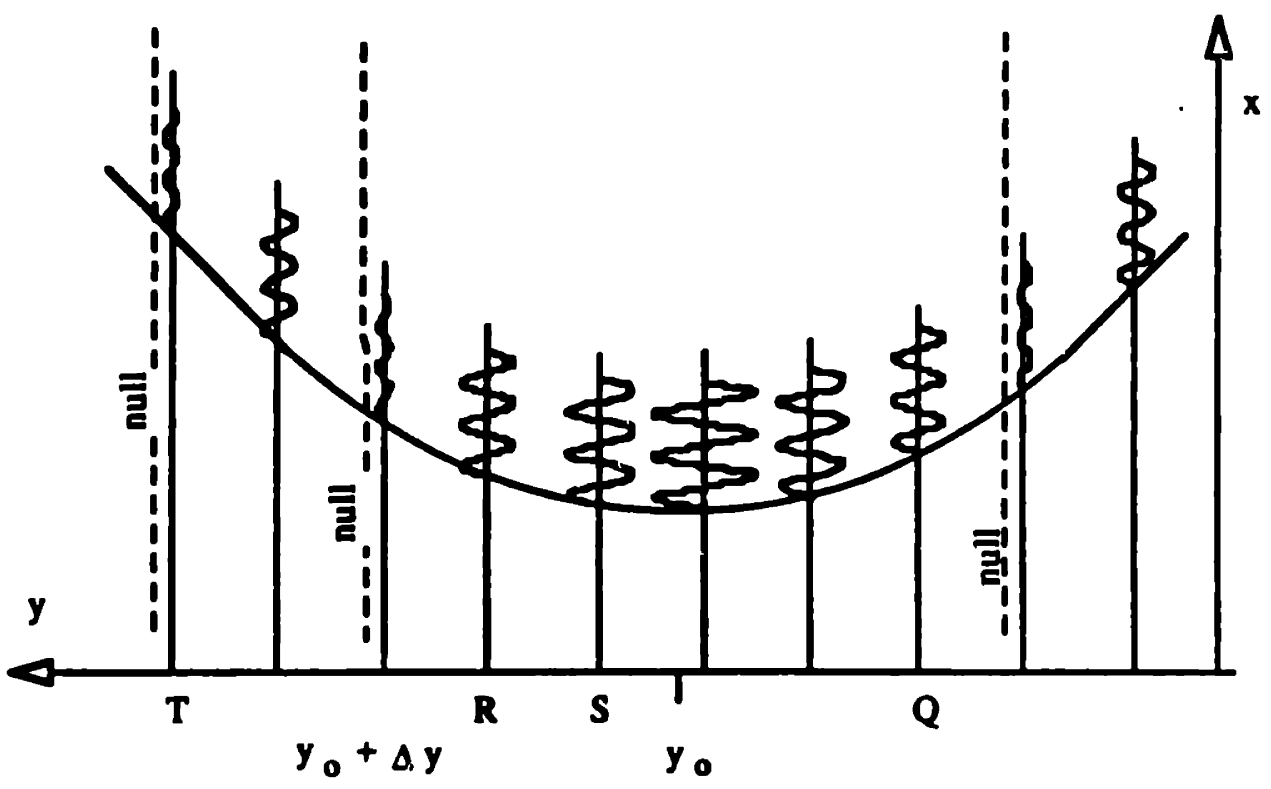

Figure 2.16 - Ensemble Time Series of Target Echoes

Showing Range Migration and Beampattern Weight 
The alias box corresponds to the image location where one of the alias target images will occur, and we determine this from Figure 2.14, part $E^{\prime}$ and from the following steps:

$$
\begin{aligned}
& \frac{P R E}{V_{0}}=\frac{1}{D_{\text {pulse }}}=\frac{1}{\lambda} \sin (\theta), \\
& \theta=\sin ^{-1}\left(\frac{\lambda \text { PRF }}{V_{0}}\right)
\end{aligned}
$$

Because the alias lobe is a result of a synthetic aperture, which has a geometric length $L$ but an effective length $L_{e f f}=2 L$, we must apply the 2-way phase coherent factor of 2 (see equation 2.20 ). Since the aperture acts as though it has length $L_{e f f}=2 L$ then the angular location of the grating lobe is $1 / 2$ the value shown by $(2.51)$, or:

$$
\dot{\theta}^{\prime}=\frac{\theta}{2}=\frac{1}{2} \sin ^{-1}\left(\frac{\lambda \mathrm{PRF}}{\mathrm{V}_{0}}\right)
$$

where $\theta^{\prime}$ is the angle of the alias lobe from the main radiation axis. The position of the alias target in the image space is given by:

$$
\Delta \mathbf{y}=\mathbf{R}_{\mathbf{0}} \tan \left(\theta^{\prime}\right)
$$

For the moment we also suppose that the platform transmits a narrowband signal, which we represent as a sine wave inside a rectangular pulse. Figure 2.16 shows the ensemble of returned echoes (the ensemble time series) for this case, showing the range migration hyperbola and the change in amplitude of the echoes. Carefully note that the ensemble shown covers the mainlobe of the length-D sonar and the first sidelobe as well. Since we have collected all the data we need to image our region, we must now decide how long our synthetic apertures shall be. Traditional synthetic aperture techniques usually use only the echoes returned through the transceiver mainlobe. Using the small angle approximation of equation 2.4 for the $-\mathbf{3} \mathrm{dB}$ width of a rectangular apenture, we again find that

$$
\mathbf{L}=\mathbf{R}_{\mathbf{0}} \frac{\lambda}{\mathrm{D}}
$$

$L$ is the geometric length for the synthetic aperture, and in this case we will say that $L$ spans 5 pulses. If we attempt to image the location of the target at $x_{0}, y_{0}$, this means we shall process the portions of the 
ensemble record centered $+/-L / 2$ about $y=y_{0}$, which means we process the time records labeled $Q$ through $\mathbf{R}$ in Fig. 2.16. First we perform a replica correlation (or pulse compression) on each time series $Q$ through $\mathbf{R}$. Each replica correlation will retum a positive number because the received echoes exactly phase match with the replica (this is expected because we are imaging a point where a target was located). This gives us an along-track array (labeled "recv") as shown in the top of Figure 2.17. For now, we don't apply any along-track taper (or weight) to this array, and so the along-track correlation (i.e. the azimuth compression) is the multiplication of this function with a "picket fence" in a rectangle (labeled the "match") and then a summation of the results. The magnitude (absolute value) of the result of the summation is the image of the point $x_{0}, y_{0}$.

We could now repeat the image process for a location at $x_{0}, y_{0}+\delta y$ where $\delta y$ is a small number; this would give the image of a point immediately next to $x_{0}, y_{0}$ on the $y$-axis. We would repeat this image process iteratively until we have imaged the entire along-track slice. We could also choose a new range value for $x_{0}$, and image an entirely different along-track slice. This is the procedure used to image an area.

Let us now repeat the image steps for the alias location, $x_{0}, y_{0}+\Delta y$. Since the range positions are the same for the target position and the alias position, the synthetic aperture length $L$ will be the same as calculated above; the only difference is that it is centered about $y=y_{0}+\Delta y$. The relevant time series for the alias position includes $S$ to $T$ in Figure 2.16. It turns out that as we progressively march from $S$ to $T$, we would notice two things: first, that the amplitude of the echo waveform approaches zero (due to the beampattern null of the sonar which had been pointed at the target (see Figure 2.15, left side); and second, that the correllation in the range direction for each time series is better and better as we approach the null (due to the phise of the received waveform progressively matching the phase of the replica better and better). Where the correlation would have been a maximum however, a null existed which crushed the waveform into oblivion. Actually, the null would never be matched exactly to the maximum alias correlation, but the very small value returned for the correlation is practically close to zero. In spite of the crushed correlation value for the pulse which would have had a maximum, there are still a large number of other time series which have significant correlation contributions together with non-zero beampattern weights. Figure 2.18 shows the results of the range compression (replica correlation in the range direction) at the top, and the null is obvious. The magnitude of the along-track correlation gives us the image value for $x_{0}, y_{0}+\Delta y$. If we plot ( $\mathrm{dB}$ scale) the remaining points along $x=x_{0}=R_{0}$ (an azimuthal slice) we observe the strong image of the target, and a weak but finite image of an alias target, as shown in 2.19. This shows that an alias target image exists in spite of the fact that the null of the sonar beampattern was supposed to completely crush the alias target. The null certainly does have an effect, but not so much as the synthetic aperture radar and sonar literalure would lead us to believe. 

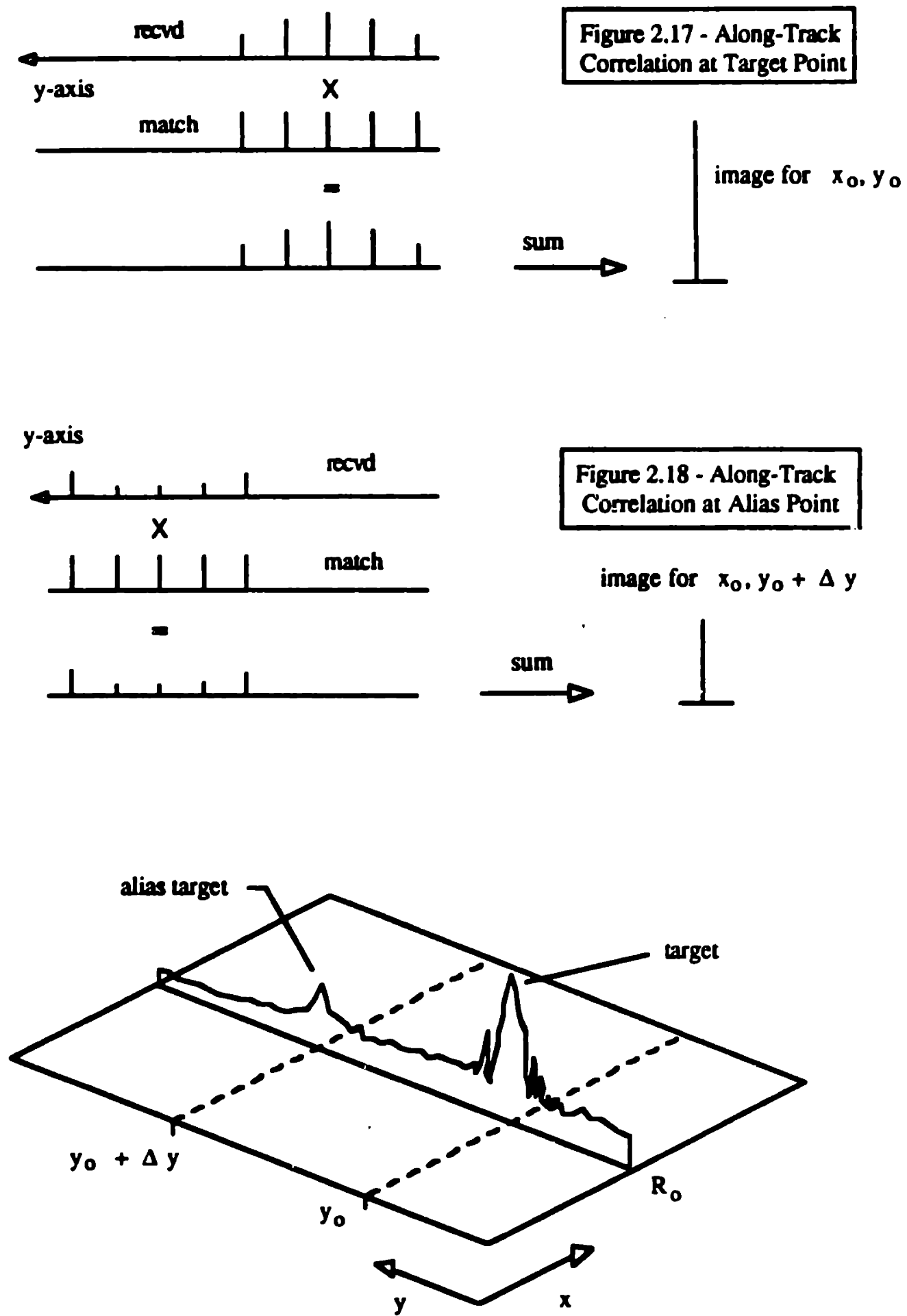

Figure 2.19 - Along-Track Image "Slice" Showing Images of Target and Alias Target 
In spite of this, SAR imagery is apparently free from defects of alias targets, and we may show how this occurs from the following reasoning. Figure 2.18 shows the results of the range compressions for the ensemble of echoes along the synthetic aperture length $\mathrm{L}$, as centered about the alias target along-track position $y=y_{0}+\Delta y$. To reduce the influence of the alias target, we see from the Figure 2.18 that reducing the length $\mathrm{L}$ would effectively shrink the along-track array; examples are shown in Figures 2.20 and 2.21 .

Figure 2.20 shows a shorter ( 3 correlation points instead of the 5 used in Figures 2.17 and 2.18). These 3 points are centered on the point we are imaging, namely $y=y_{0}$. Figure 2.21 also shows a 3 part set of along-track correlation points, only centered at $y=y_{0}+\Delta y$. By taking only 3 points along the $y-$ axis ( 3 azimuth or 3 along-track points) instead of 5 , we have shortened the length of the aperture used to form the image. We call the shortened aperture L'. From Figure 2.20 ( 3 element) we see that the value of the image point is reduced from the 5-element but we also see that the parts left out of the sum were the smallest parts. Conversely, Figure 2.21 (3 element) shows that we have used only the smallest parts of the along-track aperture to image the alias point and we have left out the strongest contributions. This causes the magnitude of the alias image to shrink faster than the magnitude for the actual target image. In the limit, if we shrink the aperture size sufficiently, we will have a vanishingly small alias image while still retaining a substantial target image. Since the entire image procedure comes from synthesizing apertures from data we have already collected, we may choose the lengths of the apertures directly in the image processing, so there is no modification to the platform, the platform speed, the sonar element size, or elsewhere.

The deliberate use of a sub-maximal length synthetic aperture accomplishes three things:

- it deletes the portions of the along-track array which strongly contribute to an alias target image;

- it reduces the magnitude of the actual target image but at a slower rate than the alias image;

- it will reduce the along-track resolution by the ratio $\mathrm{L} / \mathrm{L}^{\prime}$ (i.e. the size of the along-track resolution increases), which makes the target image appear fatter.

An illustrative example along-track slice image is shown in Figure 2.22. 

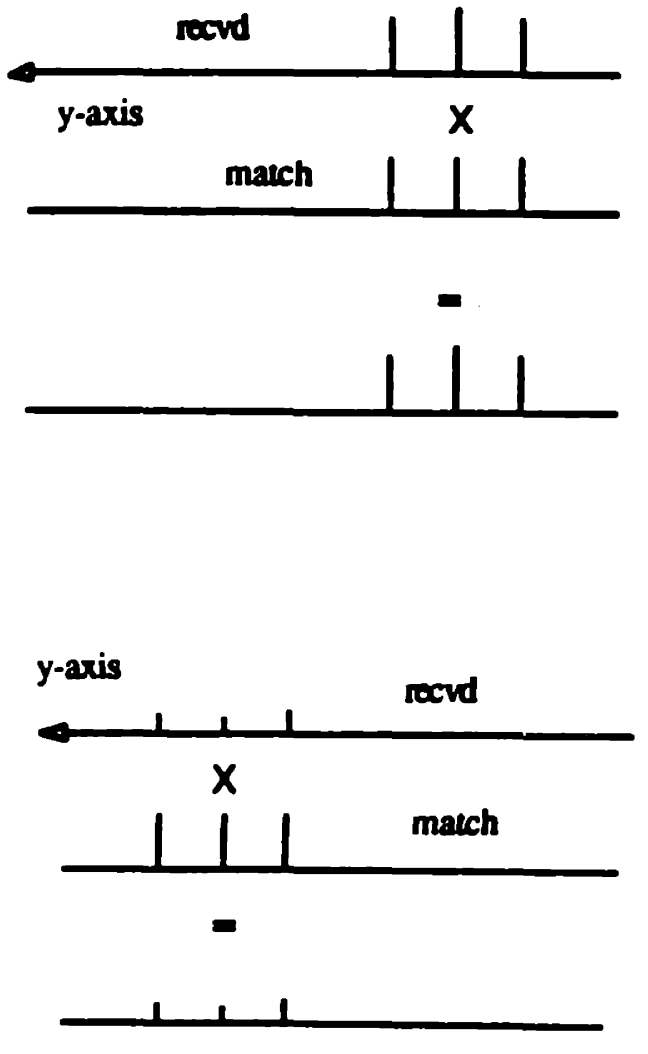

Figure 2.20 - Along-Track

Correlation at Target Point for

Reduced Aperuure

image for $x_{0}, y_{0}$
Figure 2.21 - Along-Track

Correlation at Alias Point for

Reduced Aperture

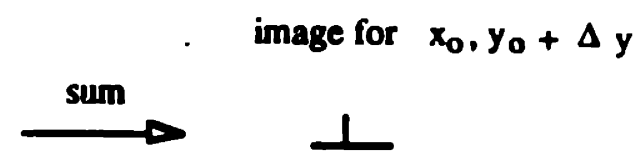

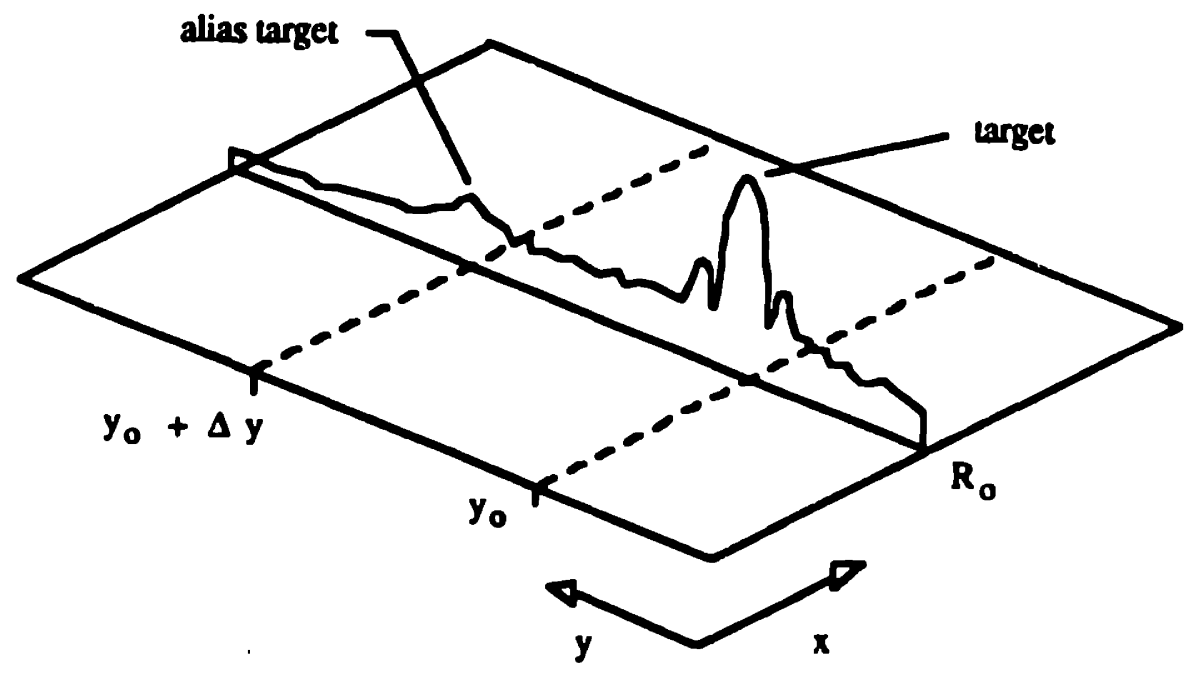

Figure 2.22 - Along-Track Image "Slice" Showing Images of Target and Alias Target for Reduced Aperure 
The tradeoff of reduced resolution for reduction of aliases is an acceptable compromise, since the total available length $\mathrm{L}$ for the synthetic aperture is frequently divided into smaller sub-apertures of equal length $L^{\prime}$. This is frequently done in SAR, but for entirely different purposes than those described in this section. The use of sub-maximum aperture lengths is always described in the SAR literature as a means to form several images of a target from several different (but identical length) sub-apertures. These images are then averaged together. The superposition of these images is an incoherent process which is used to reduce speckle (which is further discussed in 2.5.1). However, the argument presented above shows that it also reduces alias lobes in an image.

An example of azimuthal alias target lobes in a SAR image may be found in Fitch [1988], on pp. 77 81. These images show the Goldstone Deep Space Network in Califomia. The prominent star-like feature is the image of the $\mathbf{2 6}$ meter diameter antenna, and azimuthal alias lobes images of the antenna are visible in (Fitch) single look Figures 2.33, 2.34, 2.35, as well as in the four-look images (Figures 2.39 and 2.40). The alias lobe images are shown as a train of evenly-spaced bright spots in the along-track tails of the antenna image. The antenna dish was so strong a reflector that the target and alias images were much, much stronger than anything else shown in the image. If the dynamic range in the image were altered to minimize these things, the rest of the image would be essentially eliminated. On the other hand, tailoring the dynamic range in the image to show the terrain also causes the target and the alias target images to show as very, very strong targets (this is a called a cardinal effect). Note that the antenna target and its aliases were so strong that they were present in both the single look images, and in the four-look images despite the benefits of image averaging.

Five examples of spacebome SARs are shown in Table 2.2, which show that the total length $L$ of the $-3 \mathrm{~dB}$ aperture available is not fully used. Instead, the total length $\mathrm{L}$ is subdivided into smaller subapertures for the multi-look image superposition described above. The ratio $\mathrm{L} / \mathrm{L}^{\prime}$ indicates the possible number $\mathrm{N}$ separate looks that may be taken over the full $-3 \mathrm{~dB}$ to $-3 \mathrm{~dB}$ width of the main lobe of the radar antenna. The actual number of looks, $\mathbf{N}_{\text {actual, }}$ is also shown in the table. 
Table 2.2 - SAR Examples - Aperture Lengths Compared

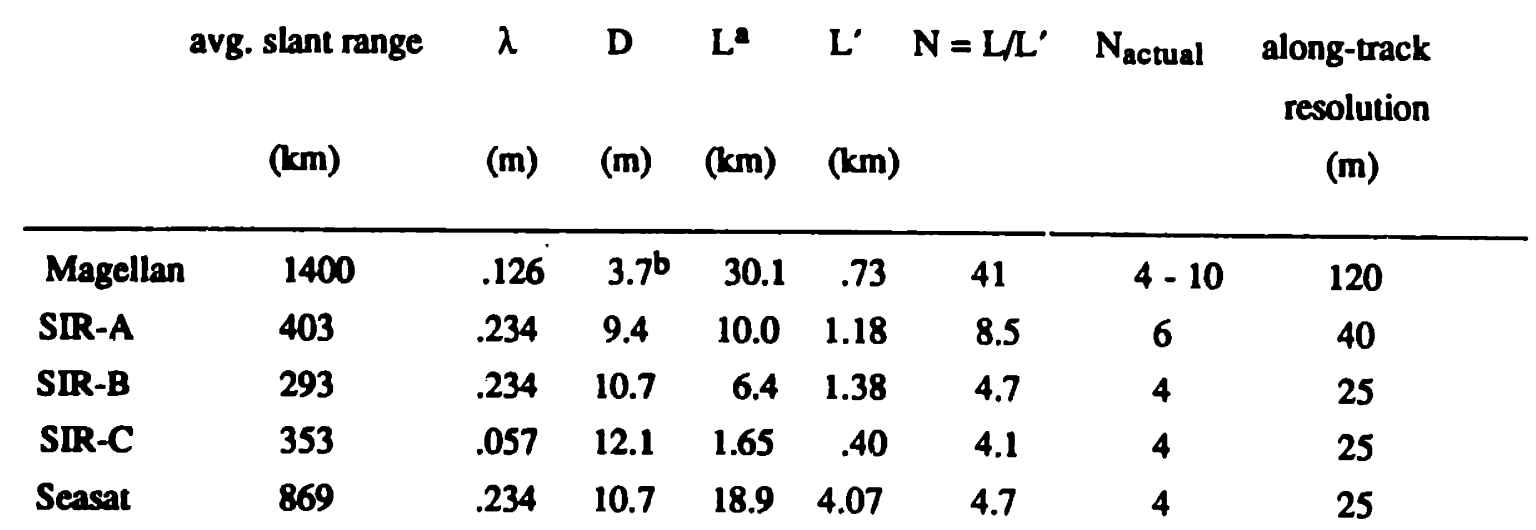

Notes: a. $L \sim R_{\mathrm{avg}} \lambda \mathrm{D}$, the maximum theoretical aperture length $-3 \mathrm{~dB}$ to $-3 \mathrm{~dB}$;

$L^{\prime}$ is the actual aperture length used.

b. circular diameter.

Magellan (1990) is a satellite designed to image the surface of Venus. SIR-A, -B, and -C (Shutule Imaging Radar, 1981, 1984 and 1991 respectively) are Earth orbiter SARs. Seasat (1978) for remote sensing of earth's oceans. Data taken from Elachi [pp. 120, 235, 1988] and Synthetic Aperture Radar, Technology and Applications [Chapter 14 by R. Shuchman, 1989].

Examples of alias target image reduction are shown in Chapter 5, which shows results for a wide variety of sonar simulations.

The influence of alias targets in the image space can also be reduced by careful use of a wideband transmit signal. This has the effect of reducing the value of the range compression along the hyperbolic arc for an alias target location because the waveforms in the wideband receive signal do not readily match the replica transmit signal. Wideband signals may be in the form of frequency modulation or FM (which is an idea mentioned by many radar and sonar papers for both real and synthetic apertures) and pseudorandom noise (see e.g. Badalyan and Bazulin, 1989). The use of broadband signals will be described in more detail in section 2.4 . 


\subsubsection{Vernier / Multiple-Hydrophone Approach to Sampling}

Several investigators in the synthetic aperture literature have mentioned the use of multiple hydrophones so that multiple beams could be formed as a way of satisfying spatial sampling. This has been called a vernier technique by Loggins [1974, 1982]; Bucknam et alia [1971] and Cutrona [1975] described the use of multiple hydrophones, whereas Gilmour [1980] specifically cited the vernier. By either name, it is best understood by giving an example of an undersampled SAS system, which is then restored to adequate sampling by the use of additional hydrophones.

Consider a synthetic aperure sonar platform which moves 100 fast (or the hydrophone array is not long enough, or the PRF is too short) so that the spatial sampling requirement is not mei because the hydrophone has moved a distance greater than $D / 2$ before the next pulse transmission. Spatial sampling may be regained by using adjacent multiple hydrophones. An example is shown in Figure 2.23 .

The system shown in Figure 2.23 works in the following way. The platform moves at speed $V_{0}$, and transmits and receives through the sonar transducer array $\mathrm{J}$. This real array sonar $\mathrm{J}$ has a total length $\mathrm{D}$, but the combination of the platform speed $V_{0}$ and the PRF cause spatial undersampling because the platform moves much more than $D / 2$ before the next pulse. To recover spatial sampling, a receive array $K$, of hydrophones located adjacent to $\mathrm{J}$ are used. Note the size of the elements in the $\mathrm{J}$ and $\mathrm{K}$ arrays are the same. Immediately after a pulse is transmitted from the array J, J becomes a hydrophone and starts recording echoes. As soon as the platform has moved some small distance (much less than D/2), the D-length receiver is electronically shifted in the aft direction. This means that one element of $\mathrm{J}$ (the forward element) is given up and replaced by the first element of $K$. This process is repeated each time the platform moves the small distance (less than $\mathrm{D} / 2$ ), and the forward-most receive element is given up and a new $\mathrm{K}$ element replaces it.

By doing this we have electronically retarded the geometric position of the active hydrophone, so that it always satisfies the sampling requirement. We continue this drop-and-add of hydrophone elements until the next pulse is transmitted, where the process starts again from scratch. A mechanical analog of this procedure would have the receive hydrophone mechanically guided along the side of the platform, and moving at a speed sufficient so that it never moves more than D/2 (in xyz global coordinates) between pulses. 


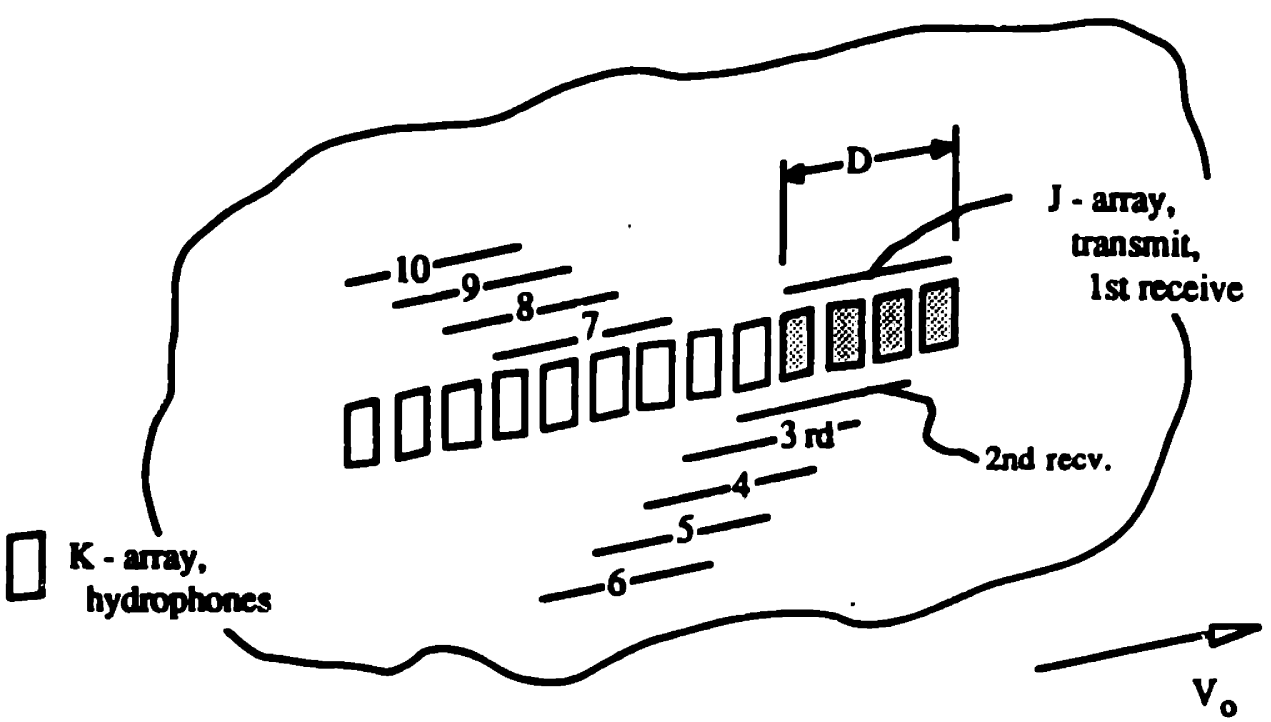

Figure 2.23 - Sequential Hydrophone Array to Satisfy Spatial Sampling 


\subsubsection{PRF Requirements, Spatial Sampling, and Maximum Range}

The spatial sampling requirement of (2.46) suggests a limit on the pulse repetition frequency, or PRF, where $P R F=1 / T$, and $T$ is the interpulse period. Since $D_{\text {pulse }}$ is the distance traveled by the platform

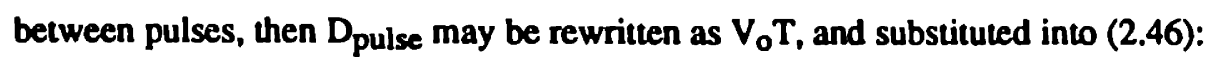

$$
V_{0} T \leq \frac{D}{2}
$$

This leads to

$$
T \leq \frac{D}{2 V_{0}}
$$

which is one requirement on $T$, and its inverse $1 / T=P R F$. The other requirement is on the maximum unambiguous range over which the sonar will operate. Each time the sonar pulses, a wave is launched into the medium (say, the ocean) which then travels at the propagation speed $c$. The wave has the opportunity to travel out to a target at the distance $\mathbf{R}_{\text {maximum, }}$ bounce off, and have the echo return to the sonar just before the next pulse is transmitted. The total elapsed time for this is

$$
T_{\text {elapsed }}=\frac{2 R_{\text {maximum }}}{c}
$$

But the pulse repetition frequency, the PRF, is the inverse of the pulse repetition period, or

$$
T=\frac{1}{\text { PRF }}
$$

Since an echo from $\mathbf{R}_{\text {maximum }}$ must return before the next pulse, this establishes the relation that $T_{\text {elapsed }}$ $<T$. We rewrite this and solve for the PRF:

$$
\text { PRF }<\frac{c}{2 R_{\text {maximum }}}
$$

This establishes the upper bound on the PRF. Equation 2.58 is also rewritten to show the lower bound on the period $\mathrm{T}$ :

$$
T>\frac{2 R_{\text {maximum }}}{C} \text {. }
$$


Equations 2.55 and 2.59 together define the bounds for $T$, which is:

$$
\frac{\mathrm{D}}{2 \mathrm{~V}_{\mathrm{o}}}>\mathrm{T}>\frac{2 \mathrm{R}_{\text {maximum }}}{\mathrm{c}}
$$

Equation 2.60 may also be rewritten for the PRF requirements:

$$
\frac{2 V_{0}}{D}<P R F<\frac{c}{2 R_{\text {maximum }}} .
$$

For both (2.60) and (2.61), the spatial sampling requirement appears on the left, and the maximum range requirement appears on the right. By way of a statement without proof (see Tomiyasu [1978], or Fitch [1988] ), it may be shown that the PRF must be greater than twice the bandwidth of the Doppler shift of a target, which is also a restatement of the Nyquist criteria.

\subsubsection{Sidelobe Levels}

The study of sidelobe levels for a synthetic aperture radar or sonar is best treated from the transform pair viewpoint (admpling domain versus beam pauern or wavenumber domain) which was previously shown in Figure 2.13. Figure 2.13 was useful for the purpose of studying sampling but was not quite accurate enough for the study of sidelobe levels, because a crucial piece of information was omitued. First, the missing information will be explained, and then a new Figure will be introduced showiiig itic correction.

In Figure 2.13 you may recall, we began with a pulse train having pulses separeted along the $y$-axis (the along-track direction for the sonar) by $D_{\text {pulse }}$ (the interpulse distance). This pulse train was then multiplied by a rectangle function, with amplitude 1.0. In order to show the length of the synthetic aperture, it was then recalled that the length $L_{\text {eff }}$ should be used for the rectangle length to correctly represent the two-way path from sonar to target and back to the sonar. The result of this step was a pulse train of delta functions beneath a rectangular window. This rectangular window pulse train was then convolved twice with a D-length, unity amplitude rectangle function which represents the real aperture of the sonar. The crucial piece of missing information is that this representation (and each item's Fourier transform) is adequate to describe a real army of length $L$, but does not contain information concerning the migration of a target acr :ss the view of the sonar length $D$, which is exactly what occurs for a synthetic 
array. So the rectangle in Fig. 2.13, item B, should be amplitude weighted by the out-and-back beam pattern of the length $D$ sonar. The out-and-back beam pattern is, of course, a sinc ${ }^{2}$ function.

Taking this approach into account, we show Figure 2.24 which has the appropriate weighting introduced across the length $L$ (note: $L$ should be $L_{\text {eff }}$ in Figure 2.24, but the notation was left $L$ for clarity in the Figure). All the items shown in Figure 2.24 are identical in meaning as those described for Figure 2.13, the only difference being the amplitude weight across the L-length rectangle function.

Note that item B shows the first nulls of the length $D$ sonar occurring at exaculy $+/-L / 2$. While this appears to be exact, it tums out that it is only approximate. The processor which turns raw pulse-echo time series data into a 2-D map of target reflectivity (i.e. the image) has a basic assumption built-in that only echoes for a target will be used if they were received through the length $D$ sonar mainlobe. However, the processor which decides whether a target echo was taken in the mainlobe usually uses the $-3 \mathrm{~dB}$ width of the mainlobe as an approximation of the null-to-null width. This is the reason why the mainlobe width has been carefully and specifically cited in this thesis as the $-3 \mathrm{~dB}$ width. The $-3 \mathrm{~dB}$ approximation has the effects of slightly wider mainlobe (and hence target along-track resolution) and slighty higher sidelobes as compared to the mainlobe and sidelobes for the null-to-null mainlobe width approximation. This is shown by the following analysis, and then by several Figures which result from the analysis.

We multiply the beam pattern weight from the sonar by the length-L rectangle, as shown in Figure 2.25. For the moment we show only the geometry, and the temporarily withhold showing the transform (wavenumber or beam pattem). Note that there are three different lengths $L_{\text {shown: }} \mathrm{L}_{\mathrm{a}}, \mathrm{L}_{\mathrm{b}}$, and $\mathrm{L}_{c}$. These different lengths correspond to the aperture length formed by the decision-Inaking rule in the synthetic aperture processing which is determined by the measure used for the width of the sonar main lobe. Note that the multiplication of $L_{a}, L_{b}$ or $L_{c}$ with the sinc ${ }^{2}$ function of the out-and-back sonar beam pattern will effectively apply different weights along the synthetic aperture length $L$. $L_{a}$ is the same length as the null-to-null spacing of the sonar beampattern. However $L_{b}$ and $L_{c}$ are shorter than the null-to-null spacing of the beam pattern, and so the result of the multiplication would cffictively 'clip' the ends off the beam pattern $\operatorname{sinc}^{2}$ function. The height of the 'clip' appears as the amplitude variable $h$ at the bottom of Figure 2.25. These different values of $L$ would naturally gives slighuly different lobe widths (and hence along-track resolution) in the transform (beam patterm) domain. Furthermore, the changing amplitude weight as a result of the sonar out-and-back beam pattern will influence both the level of the sidelobes and the width of the main lobe. 
Fourier transform $\rightarrow$

$\leftarrow$ inverse transform

A transmit pulse train

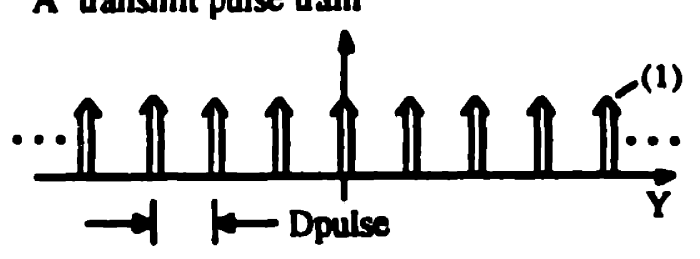

$\mathbf{x}$

B aperture length $L$

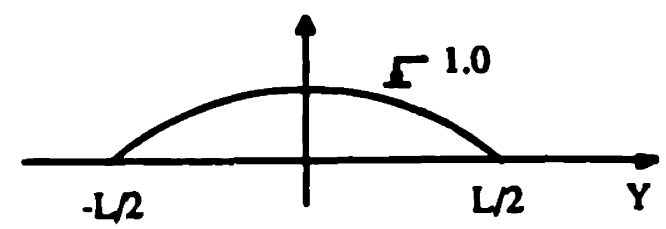

C sonar length $D$, transmit

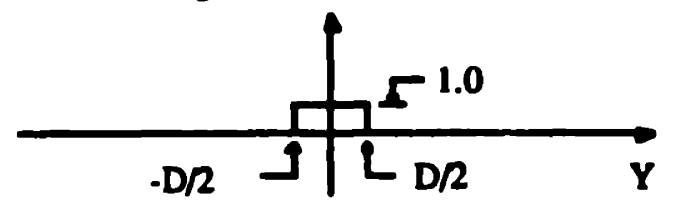

D sonar length D, receive

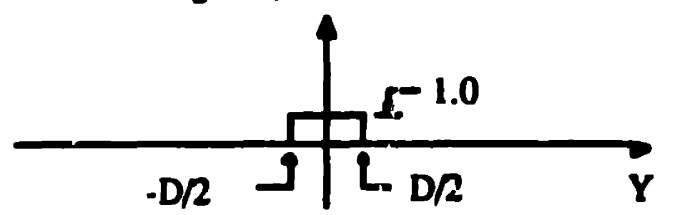

$A^{\prime}$

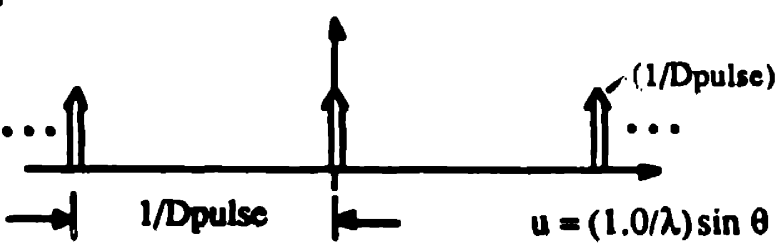

$\mathbf{B}^{\prime}$

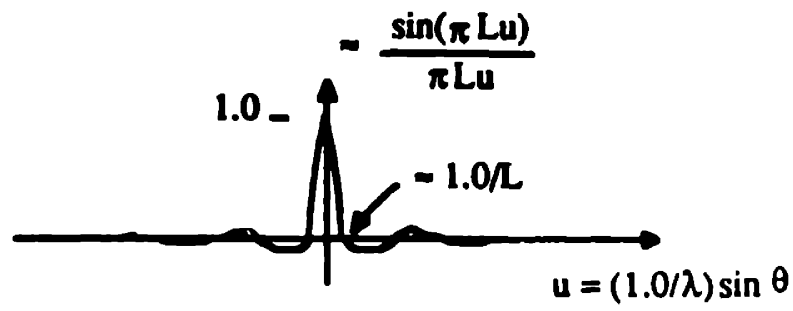

x

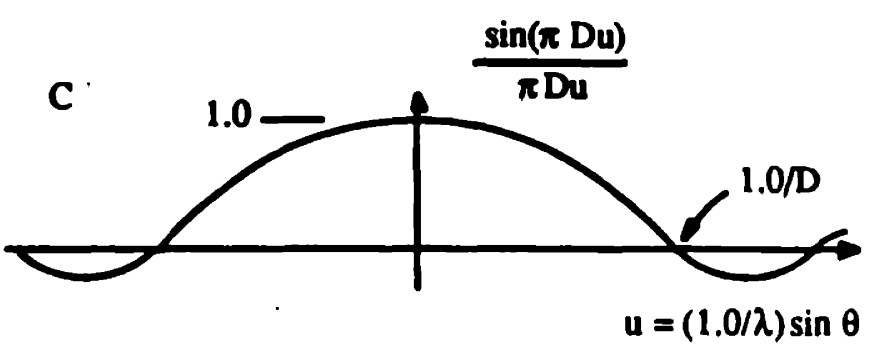

$\mathbf{x}$

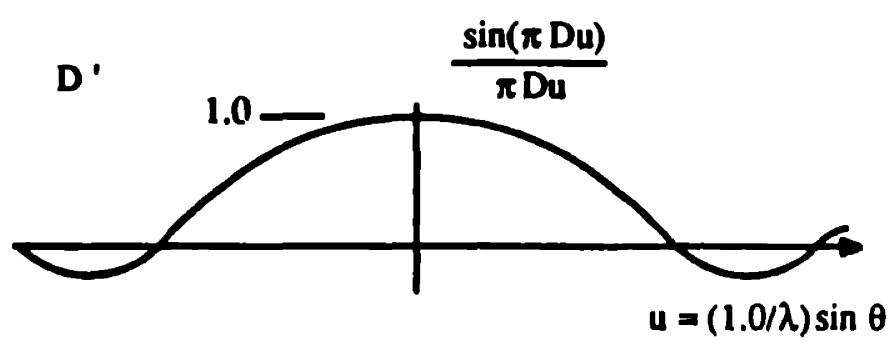

Figure 2.24 - Transform Pair: Geometry with Amplitude Weight and Wavenumber Spectra 

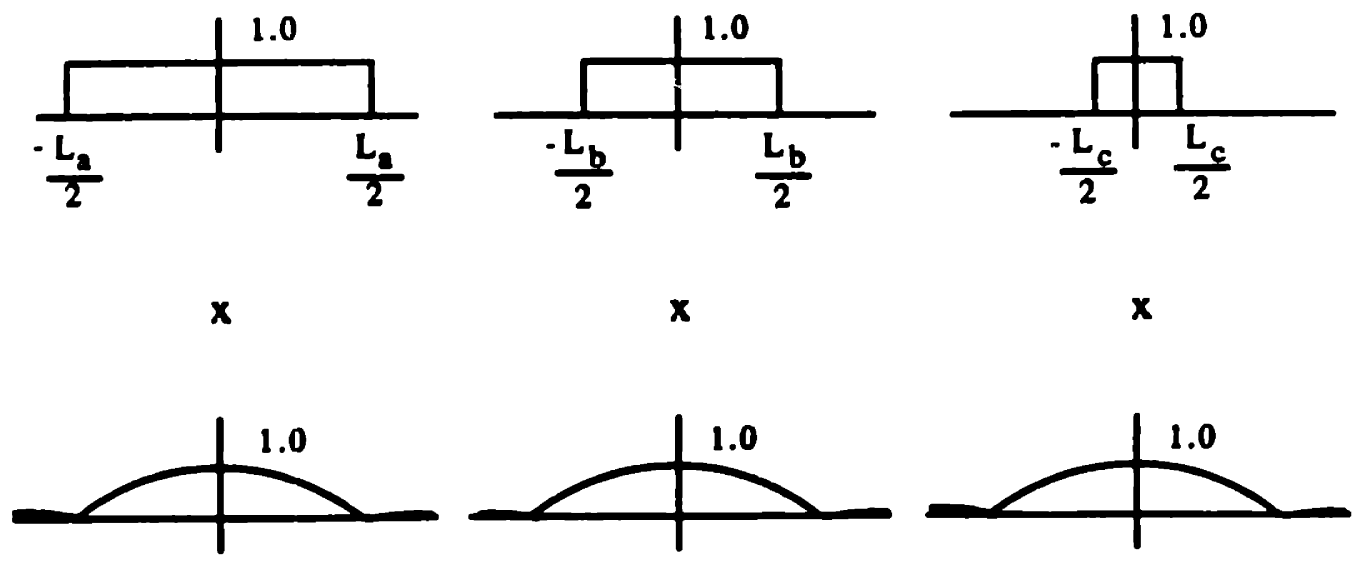

$=$
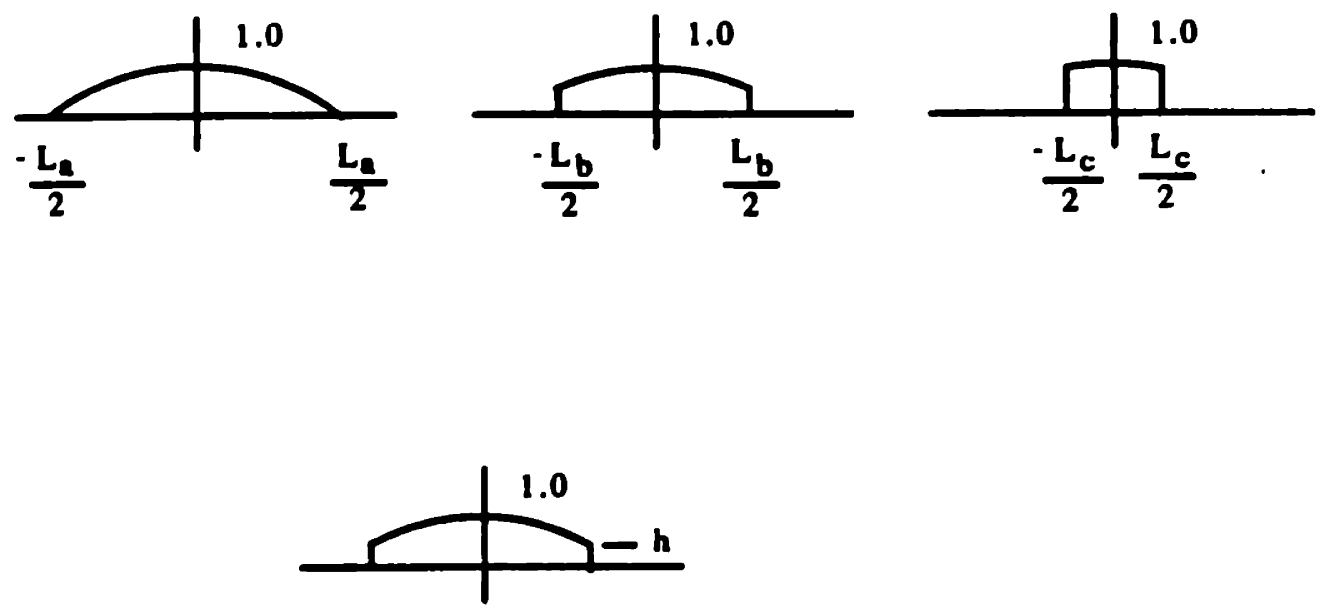

Figure 2.25 - Sonar Mainlobe Taper of

Synthetic Aperture 
Mathematically, we observe that this represents the multiplication of the L-length rectangle function with the sonar $\operatorname{sinc}^{2}$ function. We can then write the Fourier transform as

$$
B(g)=\int_{-L / 2}^{L / 2}\left(\frac{\sin \left(\frac{2 \pi y}{L_{\text {true }}}\right)}{\frac{2 \pi y}{L_{\text {true }}}}\right)^{2} \cdot 1.0 \cdot e^{-j 2 \pi g y} \cdot d y
$$

where $y=$ the geometry axis

$L_{\text {true }}=$ the true nuli-to-null length from which a synthetic aperture could be formed

$\mathrm{L}=$ the length of the rectangle $\left(\mathrm{L}_{\mathrm{eff}}=2 \mathrm{~L}\right.$ full synthetic aperture, $=2 \mathrm{~L}^{\prime}$ sub-aperture)

$$
g=(1 / \lambda) \sin \theta \text {. }
$$

The result $B(g)$ was solved numerically for several cases under the condition of a single frequency transmit signal. The criteria used was the $h$ value of the $s^{2}{ }^{2}$ function at the limits of $+/-L / 2$. Six cases were examined having the $h$ values: $0.0, .25, .41, .50, .75$, and 1.0 . The $h=0.0$ case corresponds to the null-to-null distance, $L_{\text {true, }}$ exactly equalling the rectangle length $L$. The $h=1.0$ case corresponds to uniform weighting which removes sonar beampattern; it is really a test case. The results in the wavenumber domain (beam patterns) are shown for five of the six cases in Figure 2.26 ( $h=.41$ not shown) and the sidelobe level results are shown in Table 2.3. Note that the $h=1.0$ case gives $-6.6 \mathrm{~dB}$ for the first sidelobe level (relative to $0 \mathrm{~dB}$ for the mainlobe), which is the correct result for the magnitude of the transform of a rectangle function. The $h=0.0$ case, at $-19.8 \mathrm{~dB}$, corresponds to the smallest natural sidelobe amplitude level attainable for a synthetic aperture sonar, at the expense of a wider main lobe. In comparison, Loggins et alia [1982] observed - $17 \mathrm{~dB}$ level azimuthal sidelobes in their 1982 rail-based experiment. The $h=.41$ (.407) case shown in the table is for the case where the $-3 \mathrm{~dB}$ mainlobe beampattern for the sonar is used for the weighting and gives a result of $-9.7 \mathrm{~dB}$. This agrees with the fullaperture $(\mathbb{N}=1),-10 \mathrm{~dB}$ sidelobe level observed in the SAS computer imaging model (described in Chapter 4) which uses the $-3 \mathrm{~dB}$ width across the sonar mainlobe at the center frequency of the pulse to determine the length of the synthetic array. Narrowband and broadband trials of the SAS computer model also showed an approximate first sidelobe level of about $-10 \mathrm{~dB}$ for full apertures $(\mathrm{N}=1)$, and higher sidelobe levels ( less than $-10 \mathrm{~dB})$ for sub-aperture $(\mathrm{N}>1)$ cases. 

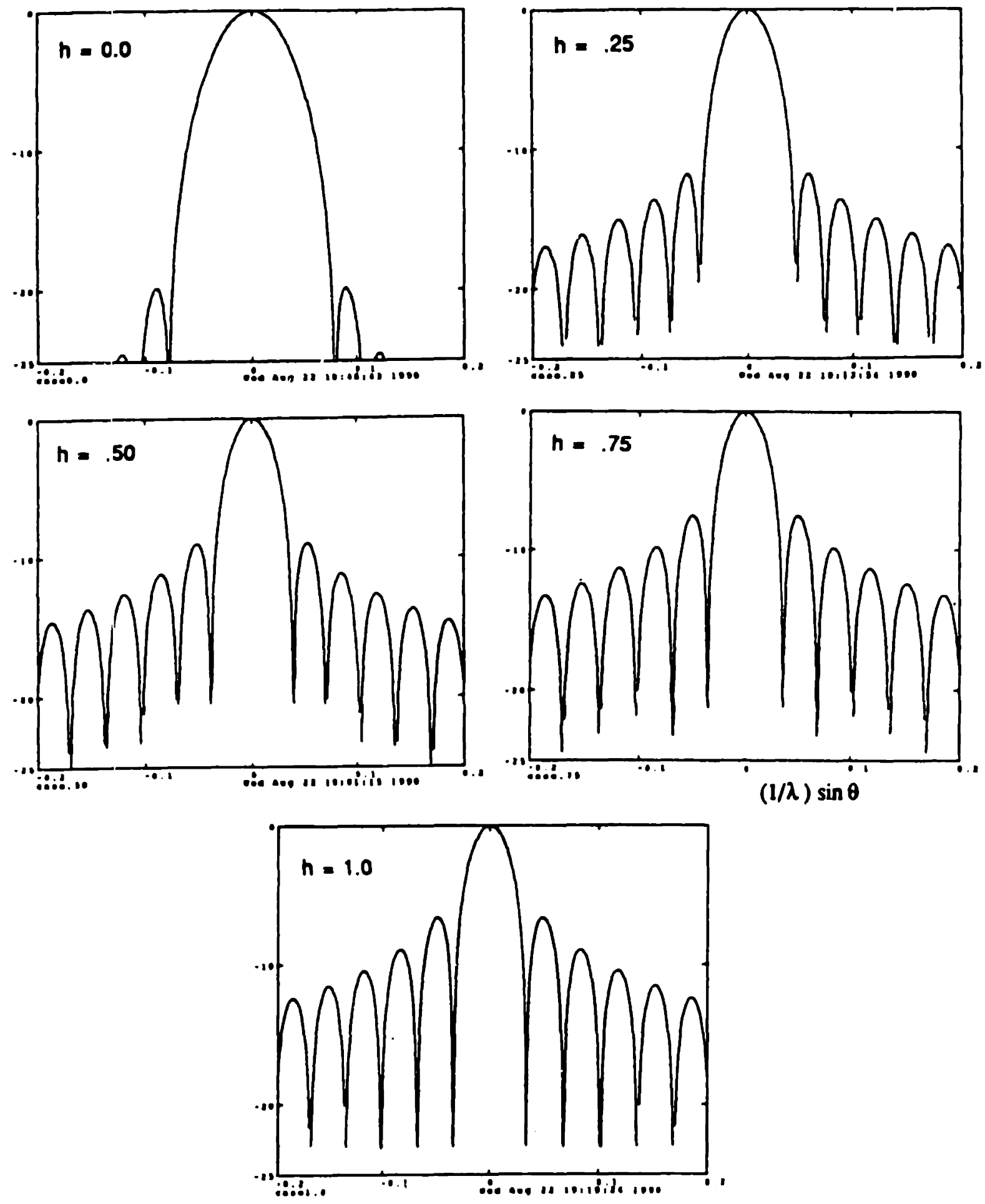

Figure 2.26 - Amplitude Taper of Sonar Beam Pattern on Mainlobe and Sidelobes of Synthetic Aperture 
Table 2.3 - Synthetic Aperture Sidelobe Levels (Natural Weighting)

\begin{tabular}{lc} 
h & $\begin{array}{c}\text { sidelobe level } \\
\text { (amplitude dB relative to the mainlobe) }\end{array}$ \\
\hline 1.0 rectangle & -6.6 \\
.75 & -7.5 \\
.50 & -9.0 \\
.41 & -9.7 \\
.25 & -11.8 \\
0.0 & -19.8
\end{tabular}

These results are general, and hold for any narrowband synthetic array which transmits and receives across a geometric length $L$, from a moving, smaller length-D sonar or radar, using only the natural alongtrack (azimuthal) weighting provided by the sonar beam pattern (see Dutkiewicz and Denbigh e.g. [1987]) and agrees with the result shown by Heimiller [Fig. 2, 1962]. Of course, extra along-tracik weighting (tapering) may be applied in the processing stages to further reduce the along-track sidelobe level. These results will also be true on-average for a synthetic aperture sonar using a wide band transmit signal. The sidelobe levels in that case would be found by an ensemble average of the wavenumber spectra (beampatuem) across the frequency band.

In summary, it may then be said that the sidelobe level, on average, for a synthetic array using the - 3 $\mathrm{dB}$ mainlobe width in the image processor is about -10 dB (magnitude of the amplitude; the power level in $\mathrm{dB}$ would be $-20 \mathrm{~dB}$ ) relative to the mainlobe, and may vary from -6.6 to $-20 \mathrm{~dB}$ depending on how the mainlobe of the length-D sonar is defined in the image processor.

\subsection{Broadband Undersampled SAS}

The previous section showed the sampled nature of a narrowband synthetic apt rture and how the discrete sampling of the aperture length $L$ along the platform path (one sonar pulse every length interval $D_{\text {pulse }}$ ) leads to definite requirements on the pulse repetition rate, or PRF. It was also shown that violations of the sampling requirement lead to alias target images and that the traditional approach was to 
'crush' the alias target lobes by (wavenumber) multiplication with the null of the length-D sonar beampauem. This section will show: the effects of spatial undersampling, how the sampling requirement may be relaxed by the use of a broadband transmit signal, how the target images appear sharp while the alias target images are smeared, and how the bandwidth relates to the level of alias target smearing (in decibels, or $\mathrm{dB}$ ) relative to the true targets.

\subsubsection{Efrects of Undersampling}

Consider Figure 2.27. This shows the geometry on the left, and the transform of geometry (i.e. the wavenumber spectra, or beam pattem) on the right. The items shown follow the same order as in Figures 2.13 and 2.24. In addition, Figure 2.27 shows the amplitude weight pattern (in item $B$ ) along the length $L$, and also shows deliberate undersampling along the $y$-axis (in item $A$ ). The widely-spaced pulses in $A$ give rise to closely-spaced pulse in $A^{\prime}$. Figure 2.28 then shows the results of the transform side of Fig. 2.27. Item $E^{\prime}$ is the result of the convolution of $A^{\prime}$ and $B^{\prime} ; F^{\prime}$ is the result of the multiplication of $C^{\prime}$ and $D^{\prime} ;$ and $G^{\prime}$ is the final result of multiplying $E^{\prime}$ and $F^{\prime}$ (this is the along-track image slice). The closely-spaced sinc functions in $E^{\prime}$ are too close to be suppressed or nulled by $F^{\prime}$, and so alias images (false targets) appear in $G^{\prime}$. This shows the effects of spatial undersampling along the $y$-axis, and the result in the image space $u$.

\subsubsection{Broadband Considerations}

The previous section assumed that the sonar transmitted either a single frequency signal, or one that is effectively a narrowband signal. Now we shall consider a broadband (or wideband) transmit signal used in an undersampled synthetic aperture sonar, and again observe the effects in the image space. We loosely define broadband for bandwidths greater than $0.1 \mathrm{f}_{\mathrm{c}}$.

Consider the case of an undersampled synthetic aperture which transmits a broadband signal. For the purposes of illustration we consider a linear FM chirp signal starting at frequency $f_{1}$ and stopping at frequency $f_{2}$ and we define the theoretical bandwidth $B W=f_{2}-f_{1}$. The pulse duration is $\tau$, and the interpulse distance $D_{\text {pulse }}=V_{0} / P R F$. We also define $p=1 / D_{\text {pulse }}=P R F / V_{0}$.

We saw from Figure 2.28 that spatial undersampling in the geometry (along the $y$-axis for example) led to alias images in the wavenumber domain, and we can always map from the wavenumber domain into an image plane by the steps shown in equations 2.50 to 2.53. Figure $2.29 \mathrm{~A}$ shows a wavenumber spectra for an undersampled synthetic aperture array for a single frequency $f_{c}$, and the alias lobes are clearly evident. 
Fourier transform $\rightarrow$

$\leftarrow$ inverse transform

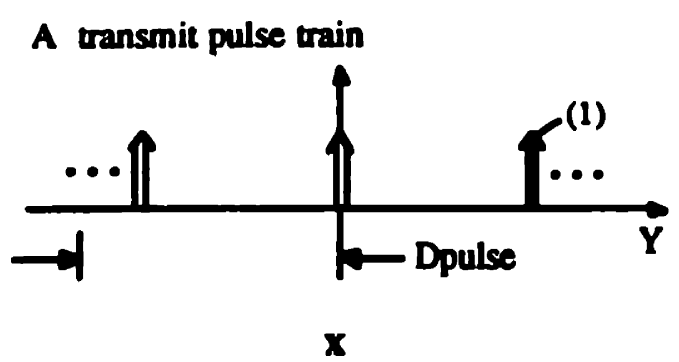

B aperture length $L$

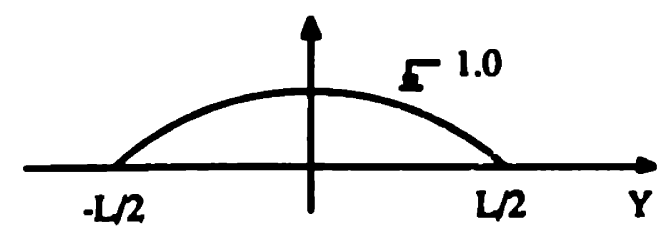

C sonar length $D$, transmit
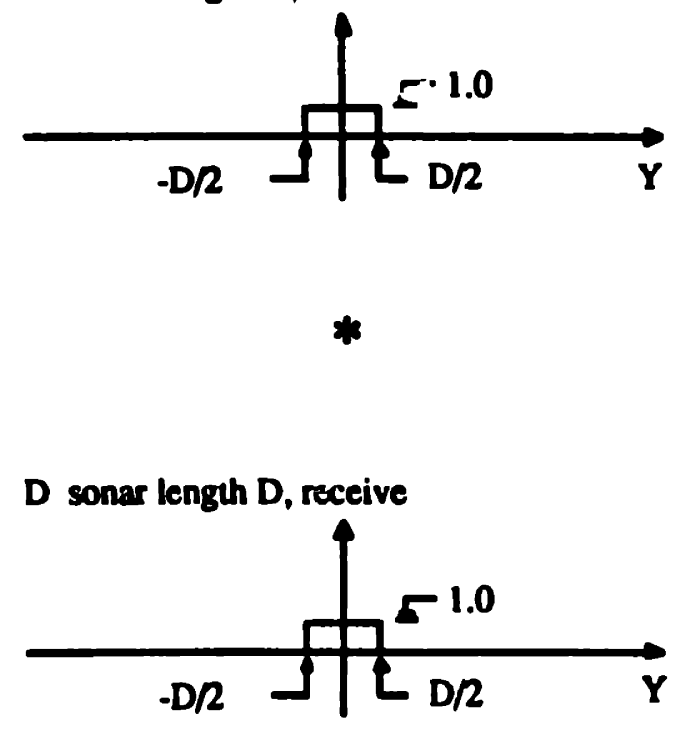

$A^{\prime}$

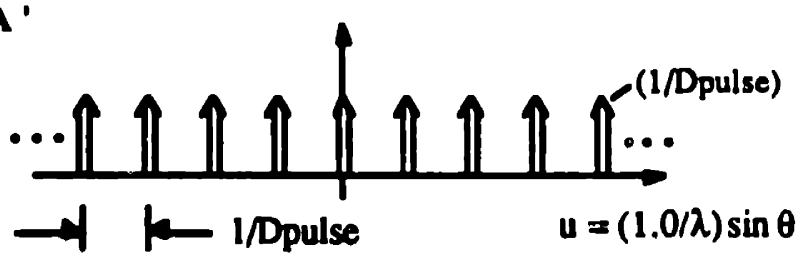

B
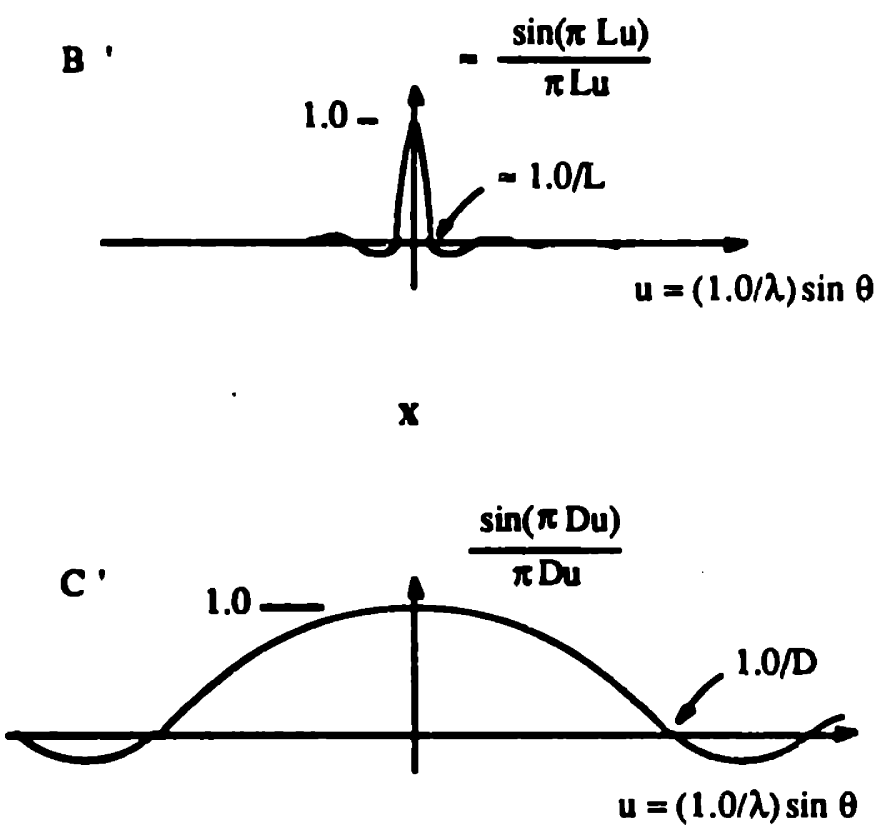

$\mathbf{x}$

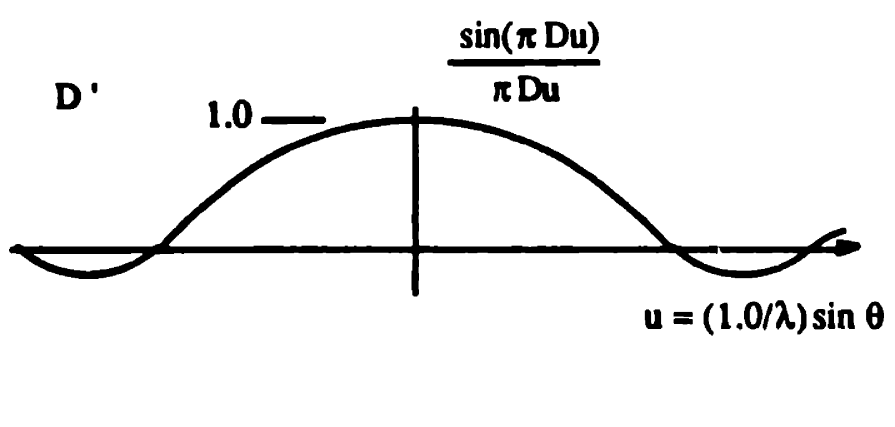

Figure 2.27 - Transform Pair. Undersampled Case 

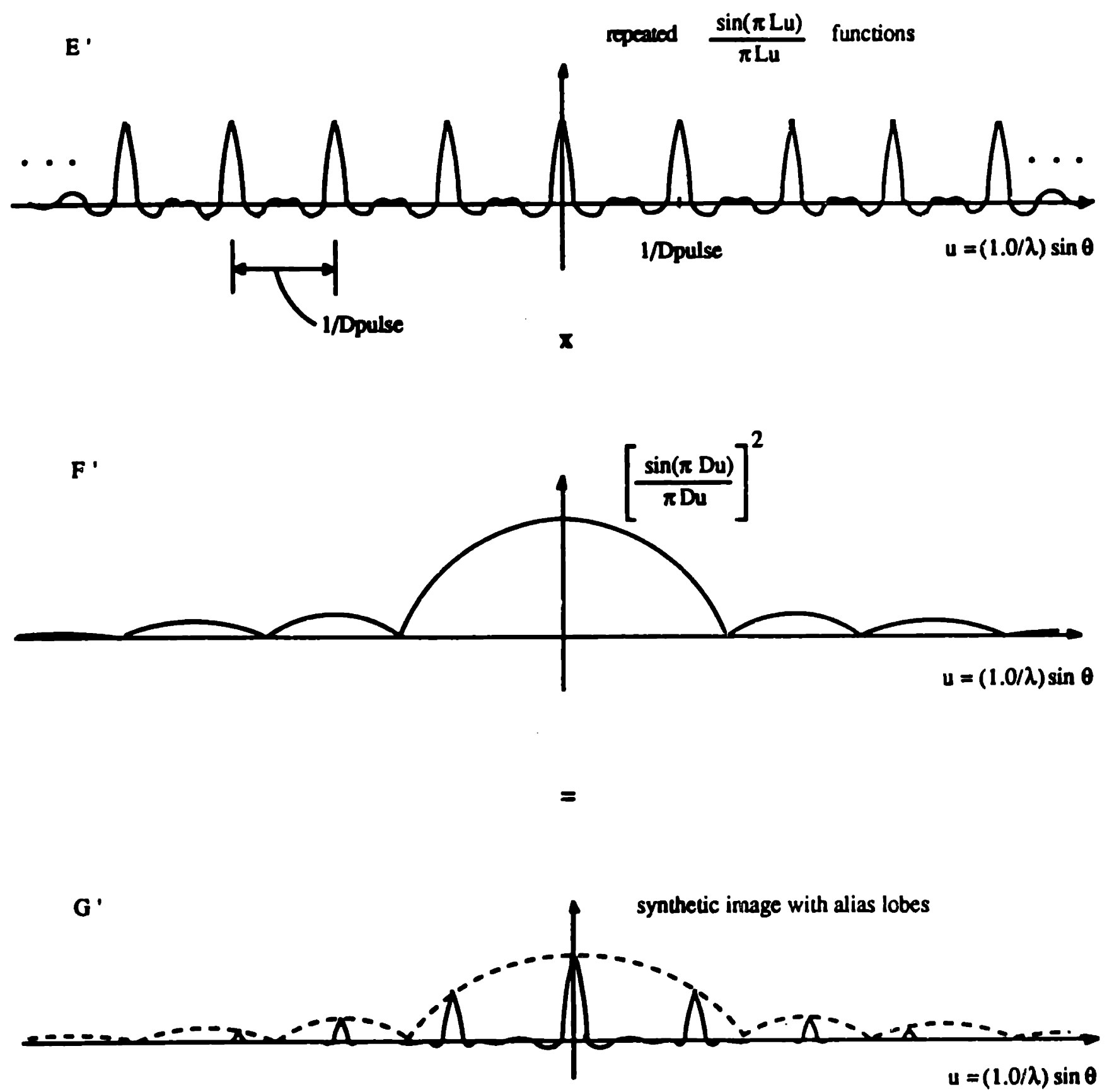

Figure 2.28 - Undersampled Case Showing Alias Images 
A. for frequency $f_{c}$

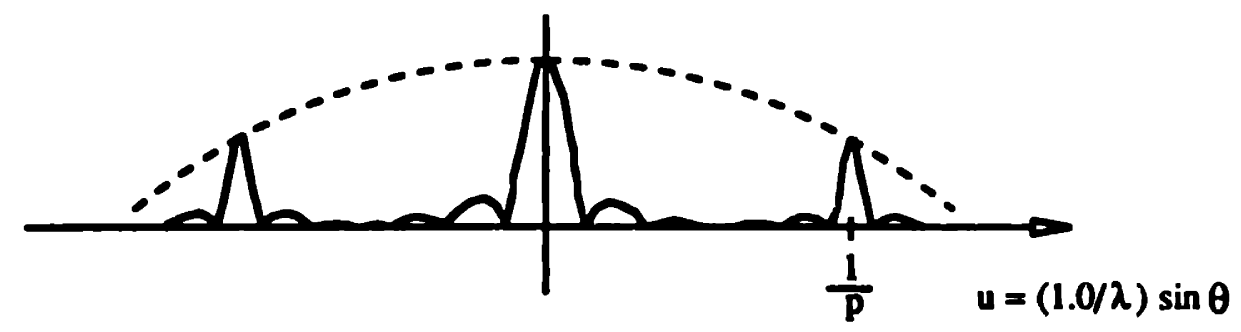

B. for transmit band

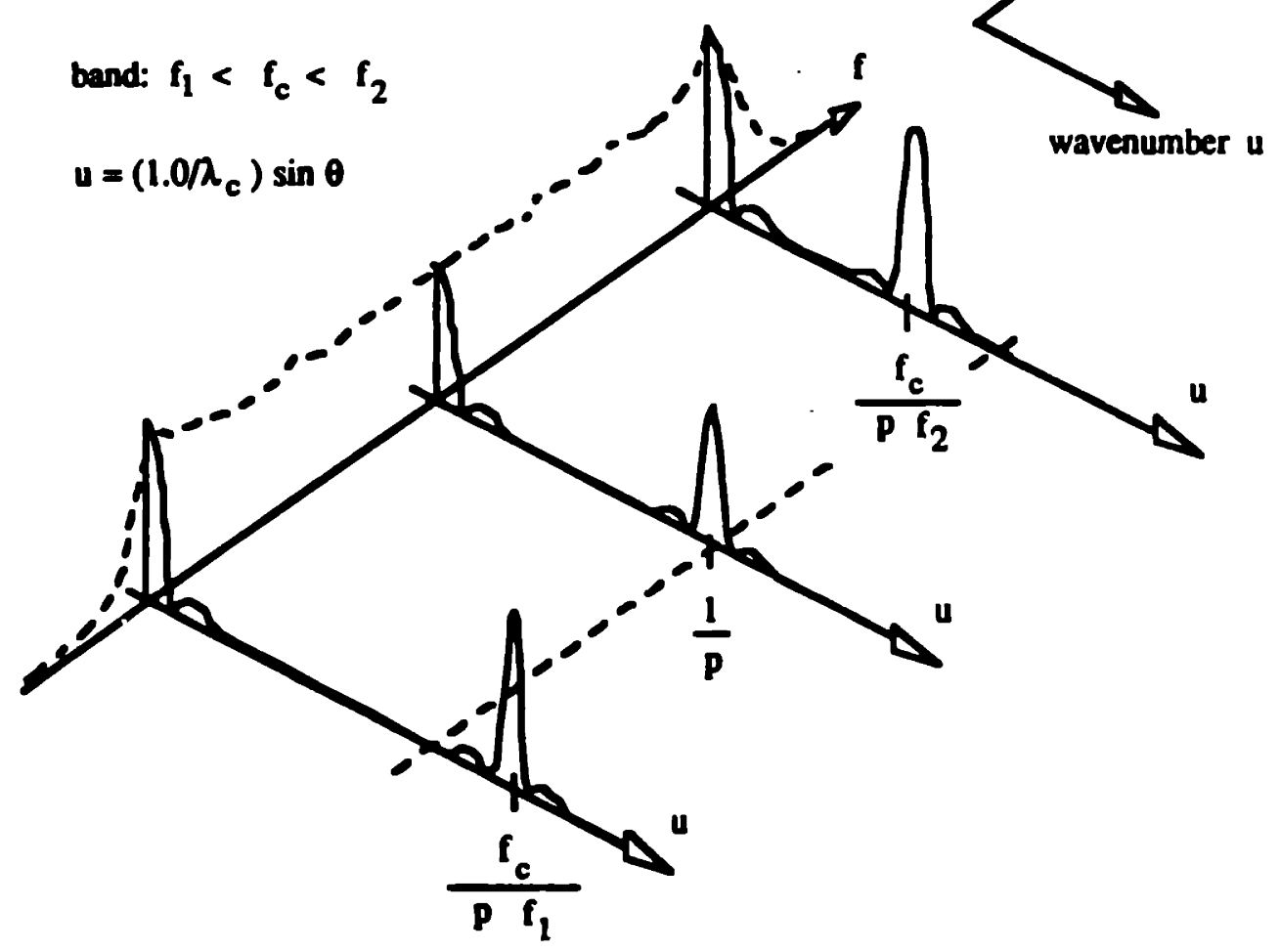

Figure 2.29 - Undersampled, Broadband Wavenumber Spectra

(or beam patterns across the transmit band) 
The dotted line in Figure 2.29A represents the weighting influence of the sonar beampattern. Note that the location of the alias lobes is inversely proportional to the wavelength $\lambda(u=(1 / \lambda) \sin \theta)$. We now show the right half plane of Figure 2.29A for three different frequencies $f_{1}, f_{c}$, and $f_{2}$, and we show them together in Figure 2.29B in an ensemble which is normalized to the same wavenumber scale $u=\left(1 / \lambda_{c}\right)$ $\sin (\theta)$. Because we have chosen a normalized scale, we observe that the alias lote for the higher frequency $\left(f_{2}\right)$ appears closer to the main lobe. Likewise the alias lobe for frequency $\left(f_{1}\right)$ appears further away from the mainlobe (the target lobe). Though we treat this ensemble for three discrete frequencies, it closely resembles the beginning, the middle, and the end of the ensemble which we would observe at $f_{1}, f_{c}$, and $f_{2}$ for a continuous FM sweep. Note also that we have temporarily ignored the influence of the sonar beampautern.

Figure 2.30 shows the dimensions for the mainlobe for $f_{c}$, and equates the actual area under the mainlobe to an equivalent rectangle with height $H$ and width $1 / L_{e f f}$, and recall $L_{e f f}=2 L$. Note that $L$ could also be replaced by $L^{\prime}$ in the following analysis for the case of sub-aperture lengths $L^{\prime}$ along the synthetic aperture length $L$ (see section 2.3.2 for discussion).

Since our objective is imaging, we must find the composite wavenumber spectra for the band of frequencies between $f_{1}$ and $f_{2}$, and this means we need to sum the ensemble across frequency. This summation has two features: first, we sum the mainlobes, and second we sum the alias lobes. Though the mainlobes have widths that vary with frequency, we may assume that over the band, the width will approximately correspond to the width of the mainlobe for frequency $f_{c}$. We may also assume that the amplitude $\mathrm{H}$ of each mainlobe in the ensemble is the same, and we reason this by inspecting some sample frequency spectra for various FM chirps. An example one-sided chirp spectrum is shown in Figure 2.31. Though this spectrum exhibits some ripple in the pass band from $f_{1}$ to $f_{2}$, for our purposes it is flat onaverage.

If we take a wavenumber sum across $f$, we will find that the mainlobes are lined-up along the same value of $u$, and arguably have the same widths (on average), so these lobes will sum and give a large tall

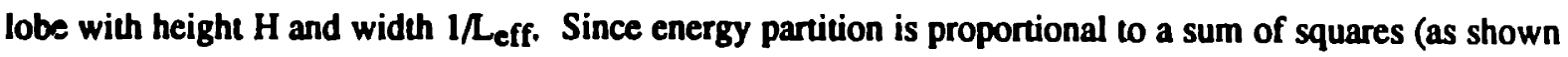
by Parseval's theorem) and since the envelope of the mainlobe peaks across $f$ is the one-sided spectrum of the transmit pulse (see the dashed line in Figure 2.29B just above the f-axis), then we must square each wavenumber spectrum and then sum across frequency. 


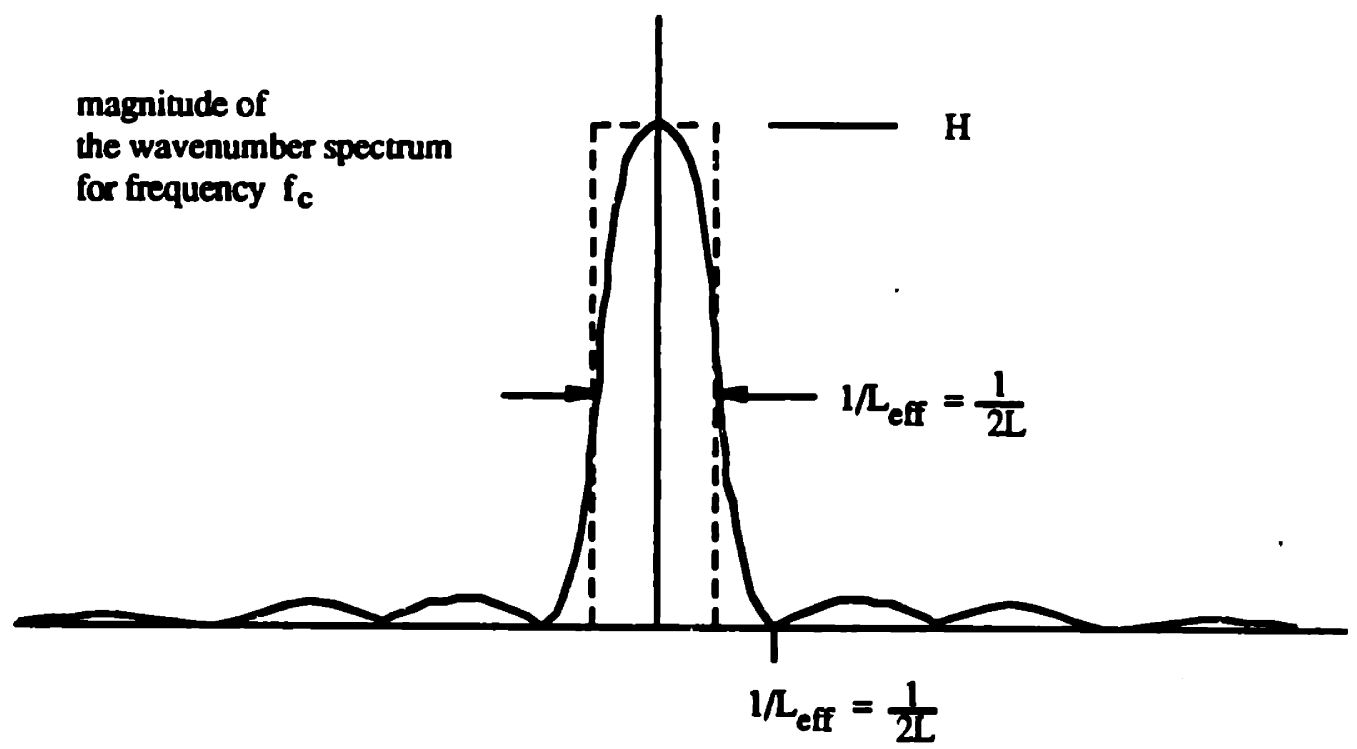

Figure 2.30 - Equivalent Mainlobe Area 


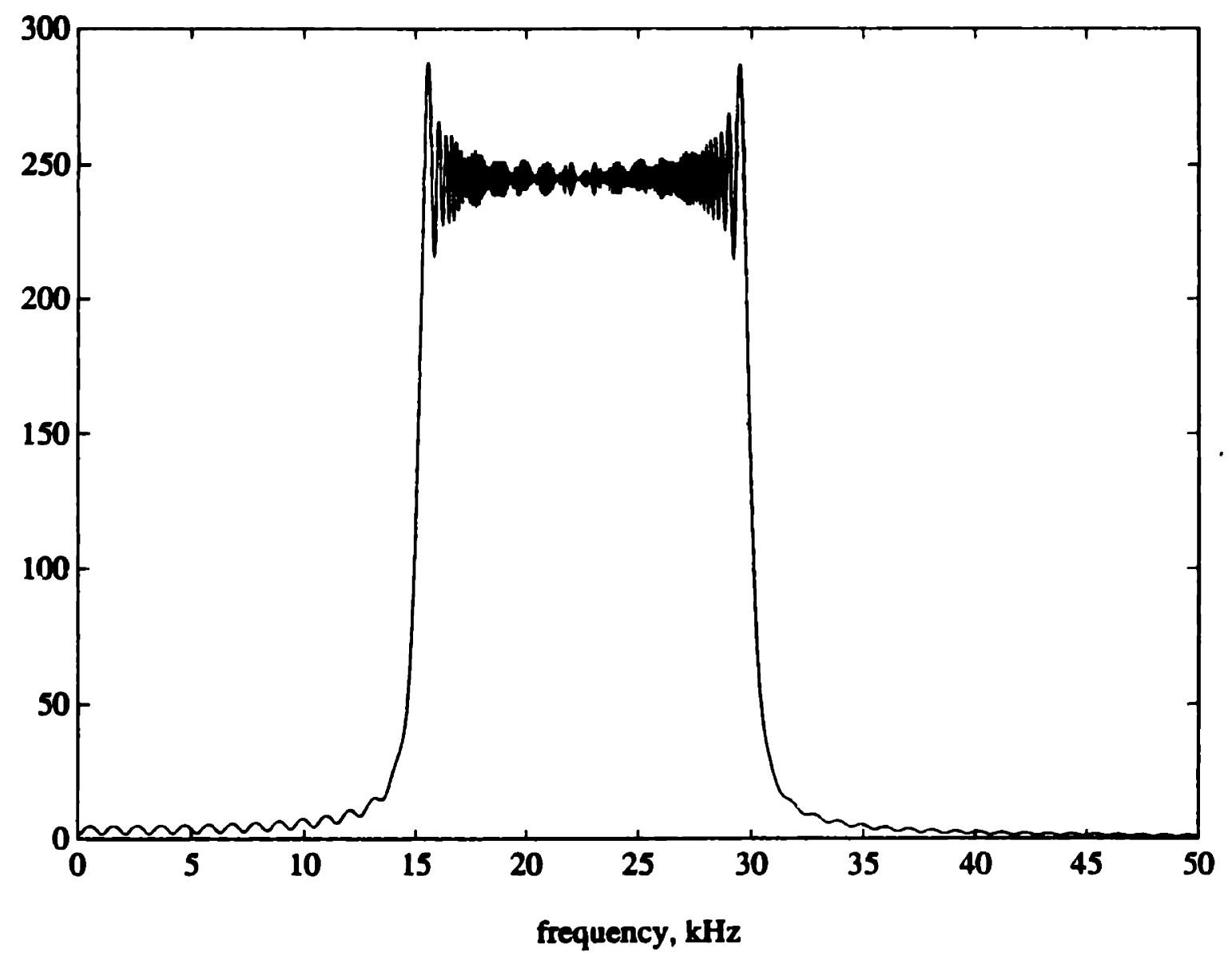

Figure 2.31 - Example One-Sided FM Chirp Spectrum 
Taking the amplitude of the mainlobe as $\mathrm{H}$, and the amplitude squared as $\mathrm{H}^{2}$, then the area under each mainlobe squared is

$$
\text { area }=\frac{\mathrm{H}^{2}}{\mathrm{~L} e f f}=\frac{\mathrm{H}^{2}}{2 \mathrm{~L}} \text {. }
$$

We then sum the area under the ensemble of squared mainlobes:

$$
\text { total area }=\frac{1}{2 \mathrm{~L}}\left[\mathrm{H}_{\mathrm{a}}^{2}+\mathrm{H}_{\mathrm{b}}^{2}+\mathrm{H}_{\mathrm{c}}^{2}+\cdots \mathrm{H}_{\mathrm{N}}^{2}\right]=\frac{\Gamma^{2}}{2 \mathrm{~L}} \text {. }
$$

The alias lobe summation is treated differently because they do not line up like the mainlobes did in Figure 2.29B. Since each alias lobe is a replica of its adjacent mainlobe, then the area under the alias lobe squared equals the area under the mainlobe squared. We then assume that the area under the sum of mainlobes equals the area under the sum of alias lobes. A composite wavenumber resembles Figure 2.32, where the shaded areas are assumed to be equal. Note that the composite mainlobe is large in amplitude relative to the smeared ensemble of alias lobes. This shows how a target viewed by the composite mainlobe stays sharp and the alias image lobes are smeared. We find the area of the smeared alias lobe shaded region by multiplying the approximate width of the base by the height. The width and height are respectively:

$$
\begin{array}{r}
\text { width }=\frac{P R F f_{c} B W}{V_{0} f_{1} f_{2}}, \\
\text { height }=H^{2} .
\end{array}
$$

where

$$
f_{c}=\frac{f_{2}-f_{1}}{2}
$$

With no loss of generality we may assume that the amplitude $\mathrm{H}=1.0$ for a normalized beampattern. The area of the alias smear is approximately the product of $(2.65)$ and (2.66), which we then equate to (2.64) and solve for the unknown $\Gamma$ :

$$
\Gamma=\sqrt{\frac{2 L P R F f_{c} B W}{V_{o} f_{1} f_{2}}} .
$$




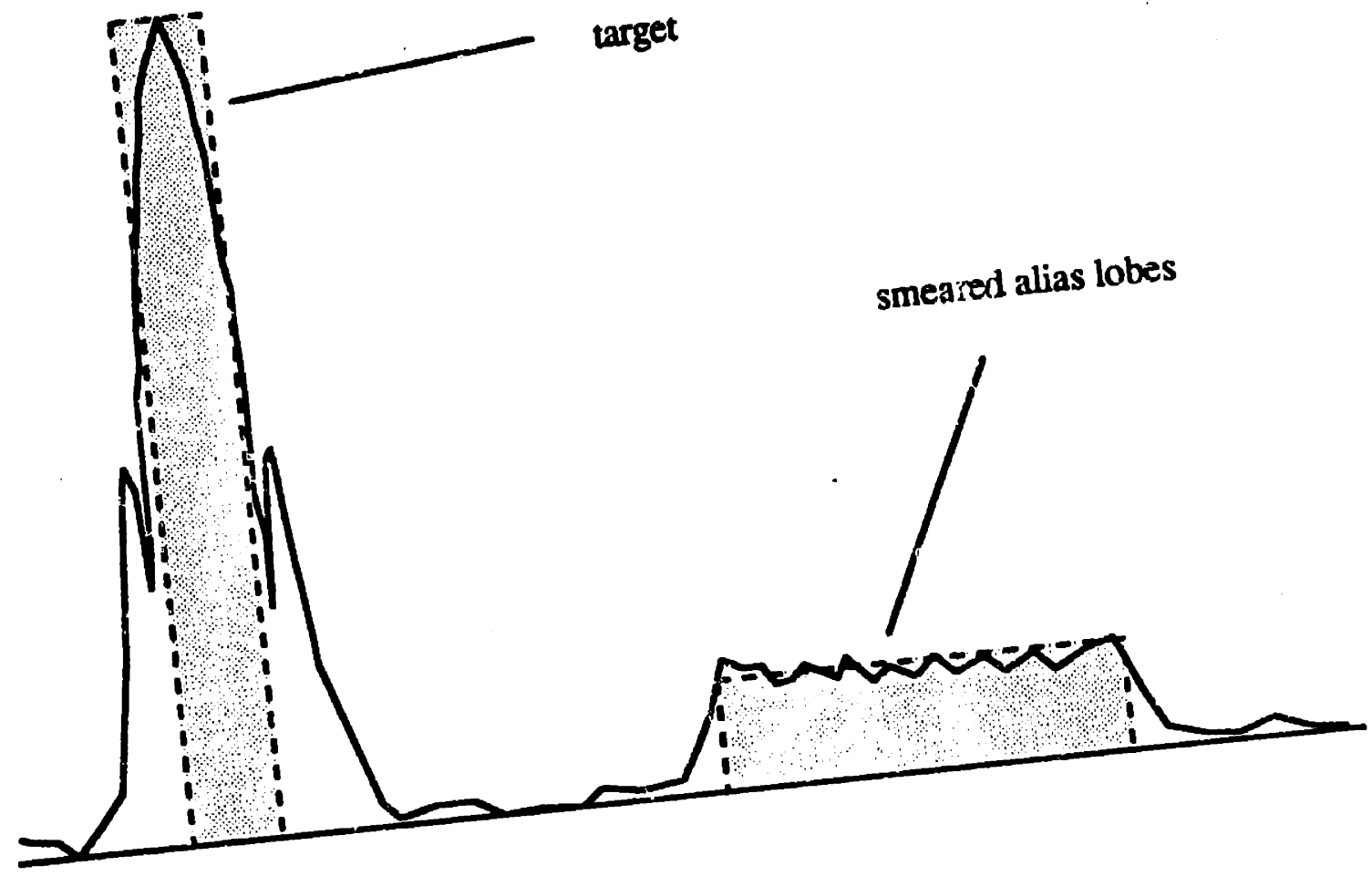

Figure 2.32 - Composite Wavenumber (beampattem) Spectrum for Broadband Undersampled SAS 
$\Gamma$ is the amplitude of the composite mainlobe relative to an alias lobe smear level of 1.0 based on a partition of the sum of squares of mainlobes across an ensemble of frequencies $B W=f_{2}-f_{1}$. An implicit feature in (2.67) is that the transmit pulse duration $\tau$ is sufficiently large (and that $1 / \tau$ is small relative to $B W$ ) that the spectrum of the transmit pulse is essuntially flat from $f_{1}$ to $f_{2}$.

We may now renormalize the amplitude of the composite mainlobe to 1.0 which, in turn, makes the level of the alias smear lobes $1 / 5$.:

$$
\begin{aligned}
& \qquad \frac{1}{\Gamma}=\sqrt{\frac{V_{o} f_{1} f_{2}}{2 L P R F f_{c} B W}}, \\
& \text { where } B W>\frac{V_{o} f_{1} f_{2}}{2 L P R F f_{c}} .
\end{aligned}
$$

Equation 2.70 places the absolute minimum requirement on the bandwidth needed to ensure that the alias will be smeared (and that the term inside the radical in (2.69) is less than 1.0). In practice however, to realize any useful gain from wide hand operation as a means of reducing the effect of alias image lobes in an undersampled aperture, the bandwidth BW must be at least a factor of 10 beyond the requirement of (2.69), and in general,

$$
B W \gg \frac{V_{o} f_{1} f_{2}}{2 L P R F f_{c}}
$$

These considerations rely on the fact that the alias lobes migrate across a normalized, $\left(1 / \lambda_{c}\right) \sin \theta$, wavenumber axis during the linear FM sweep, while the mainlobe is stationary. The cumulative effect, once we form images, is that the main lobes add constructively and the alias lobes smear across the image plane. This is a well-know result for both real aperture arrays (see e.g. Macovski, Fig. 11 [1979]) and synthetic aperture arrays.

To put (2.69) into a more useful form for the sonar designer, we make several substitutions. First we We substitute $L=R_{0} \lambda D / D^{2}$ into (2.69); this gives a $V_{0} / D$ term in the expression which we need for the second step. Then, in the second step, we must account for the possibility of processing subaperture lengths to reduce azimuthal ambiguities. If we allow the full aperture length $\mathrm{L}$ to be processed to form an image, then it may be shown that the full Doppler shift for the target is given by $\mathrm{V}_{\mathrm{o}} / \mathrm{D}$ using the small 
angle approximation; since we allow the opportunity of processing less than the full aperture length $\mathrm{L}$, then the Doppler shift for the target is given by

$$
\text { Doppler shift for subaperture }=\frac{\mathrm{V}_{\mathrm{o}}}{\mathrm{ND}}
$$

where $\mathbf{N}$ is the number of subapertures (or looks) along $\mathrm{L}$, and the Doppler shift is in $\mathrm{Hz}$. We see from (2.72) that the Doppler shift for subaperture processing is the full Doppler shift divided by the number of subapertures. We simply then substitute (2.72) in place of $V_{0} / D$ to give use the more useful result:

$$
\frac{1}{\Gamma}=\sqrt{\frac{D V_{0} f_{1} f_{2}}{2 N R_{0} \lambda_{c} P R F f_{c} B W}} .
$$

We then take $10 \log _{10}(1 / \Gamma)$ to find the level of bandwidth smear in decibels:

$$
L_{\text {BWs }}=5 \log \left(\frac{D V_{0} f_{1} f_{2}}{2 N R_{0} \lambda_{c} P R F f_{c} B W}\right),
$$

$$
\begin{aligned}
& \text { where } \quad N=\text { the number of sub-aperture lengths along } L ; L=N L^{\prime} \\
& L=\text { the length of the full aperture }=R_{0} \lambda_{d} / D \\
& D=\text { the sonar along-track (azimuthal) length } \\
& \mathrm{V}_{\mathrm{o}}=\text { platform speed }(\mathrm{m} / \mathrm{s}) \\
& f_{1}=\text { lowest band frequency }(\mathrm{Hz}) \\
& \mathrm{f}_{2}=\text { highest band frequency }(\mathrm{Hz}) \\
& \mathbf{R}_{\mathbf{o}}=\text { the broadside slant range to a target point } \\
& \mathrm{PRF}=\text { pulse repetition frequency }(\mathrm{Hz}) \\
& f_{c}=\text { center frequency }(\mathrm{Hz}) \\
& \lambda_{c}=c / f_{c}=\text { the arithmetic center wavelength ( } c \text { is the local sound speed) } \\
& B W=f_{2}-f_{1}(H z) \text {, or bandwidth, }
\end{aligned}
$$

and where LBWS is the level of bandwidth smear of alias lobes in $\mathrm{dB}$ relative to the mainlobe for a target, for an undersampled synthetic aperture sonar. 
Equation 2.74 is not valid when the synthetic aperture is sampled at the Nyquist rate because the influence of the sonar beampattern in the neighborhood of the sonar beampattern null is not taken into account. It will also be shown in Chapter 5 that $\mathrm{N}$ should always equal 1 for the broadband undersampled synthetic aperture. Attempts at contriving a "ballpark" equation for an estimate on the alias level vere unsuccessful using (2.74) in combination with the sonar beampattern function, especially in the region of the beampattern nulls. Detailed exploration of alias levels and sidelobe levels is of course best left to the computer model, but equation 2.74 serves for quick calculations.

It would also be useful to the sonar designer to have an estimate on the necessary transducer acoustical quality factor $Q_{a}$, where $Q_{a} \approx f_{c} / B W$. We substitute (2.70) into this expression for $Q_{a}$ and use $R_{n} \lambda_{c} / N D$ for the synthetic aperture length, and assume that $f_{1} f_{2} \approx f_{c}$ :

$$
Q_{a}<\frac{2 R_{0} \lambda_{c} P R F}{D V_{0}}
$$

where $D$ is the length of the sonar element $\left(D_{\text {sonar }}\right)$, and $N=1$.

Equation 2.75 is nearly identical to equation (3.3.2-8) in de Heering [1989] which is, apart from the typographical error in his thesis, repeated here as:

$$
Q \leq \frac{2 \alpha \mu \lambda_{0} R}{D_{\text {pulse }} D_{\text {sonar }}}
$$

where " $\alpha \leq 1$ accounts for shading effects," " $\mu \leq 1$ is a spatial oversampling factor," ambiguities (aliases) occur whenever $\mu<1.0$, and $\lambda_{0}$ is the square root of $\lambda_{1} \lambda_{2}$. The fact that de Heering's approach was different than the one shown here lends credibility to both results. de Heering's analysis also stated that for the alias lobes (he refers to them as grating lobes) to be completely blurred over the image plane requires that

$$
\lambda_{1} \geq 2 \lambda_{2} \text {, or that } Q \leq 1.4 \text {. }
$$

This result, however, does not given an indication of the level to which the alias lobes are smeared, and hence motivated the search for the ballpark equation 2.74. For further details, see section 3.3, pp. 4752 in de Heering [1989]. 
As an illustration, if we desire a $-10 \mathrm{~dB}$ smear level per equation 2.74 (which approximately equals the level of the first mainlobe sidelobe for an natural beampattern taper synthetic array using the full length $\mathrm{L}$, or $\mathrm{N}=1$ ) the $B W$ and $Q_{a}$ are given by

$$
\begin{aligned}
\mathrm{BW} & \geq \frac{50 \mathrm{DV} \mathrm{V}_{\mathrm{o}} \mathrm{f}_{\mathrm{c}}}{\mathrm{R}_{\mathrm{o}} \lambda_{\mathrm{c}} \mathrm{PRF}}, \\
\mathrm{Q}_{\mathrm{a}} & \leq \frac{\mathrm{R}_{\mathrm{o}} \lambda_{\mathrm{c}} \mathrm{PRF}}{50 \mathrm{DV} \mathrm{V}_{\mathrm{o}}},
\end{aligned}
$$

where $f_{1} f_{2} \approx f_{c}^{2}$.

In review, the consequences of broadband operation are two-fold:

- it provides a means for pulse compression and hence gives good range resolution (as shown in Appendix B);

- it permits the use of an undersampled synthetic aperture that is, within the limits shown by (2.71), free of alias targets to a level of $L_{B W S}$ (in $\mathrm{dB}$ ).

\subsection{Signal Processing Options}

This section discusses three types of signal processing options: multi-look, hybrid, and 3-D. Multilook processing is widely used today in SAR, but hybrid and 3-D processing have been seldom mentioned and rarely implemented.

\subsubsection{Multi "Look" Processing}

Images resulting from coherent processing, like those of SAR and SAS, occasionally suffer from speckle. Speckle is a term to describe the changing granularity in an image. Speckle is analogous to closely viewing a newspaper picture which is comprised of tiny black or white pixels. Viewed close-up, the picture is very grainy, and if viewed extremely closely (a few centimeters e.g.) the object in the picture will be entirely obscured. However, by viewing the same newspaper picture from a distance, the view is naturally averaged and hence appears sharper, even though there is an accompanying loss in resolution. Speckle acts likes the newspaper graininess, but it comes from imaging objects that are smaller than the size of the resolution cell. This gives rise to phase interference patterns in the image which give the image 
a grainy appearance. One way to simulate speckle in the computer model is to place two point targets next to each other, and separate them by a distance smaller than the smallest pixel-to-pixel distance (i.e. the size of the resolution cell). The resulting image will only show one target in the cell containing the two point targets, but it will also show an interference pattern outside the target cell.

There are two ways to reduce speckle (or coherent speckle). One way is to collect two (or more) sets of reflection data for a target area, either by separate fly-bys or by having two real apertures available on the platform to collect data. Images may then be found for each one data set and then all the images are averaged together. This will tend to reduce the speckle but maintain the resolution.

The second way only requires a single fly-by but suffers a loss in resolution. In this case, the synthetic aperture length $L$ is divided into $N$ sub-aperture lengths, and a target image is formed from each sub-aperture length. The images are then averaged, which like the previous method, will reduce the speckle because the phase interference pattern will not be necessarily the same for each sub-aperture. However, because each sub-aperture is $1 / \mathrm{N}$ the length of the full aperture length $\mathrm{L}$, then the along-track (azimuthal) resolution will be degraded by a factor of $\mathbf{N}$. See also section 2.3 .2 for a related discussion on the influence of multilooks on alias lobe levels for sampled synthetic apertures. Examples of speckle reduction at the expense of resolution are shown in several of the SAR texts listed in Chapter 1.

\subsubsection{Hybrid Focused-Unfocused Processing}

Previous sections discussed the foctised, unfocused and spotlight varieties of synthetic arrays. Each one of these methods demands different computation efforts to form an image at a single point. The spotlight array, for example, could come from an array of theoretically indefinite length, which in turn implies a large number of echoes for coherent processing, which requires more computing effort. The focused array probably requires fewer echoes to process than the spotlight for a given image point, and similarly the unfocused has even less data to handle. Its is always useful to reduce computation and processing efforts wherever possible, and to strive for real-time imaging performance. Since the synthetic aperture sonar might be operated in regions where there are few strong targets, it would be useful to establish a processing hierarchy which would adaptively select unfocused, focused, and perhaps even spotlight in an automatic fashion during the processing. For example, a survey is taken in a bay to find wrecks in an otherwise sandy-bottom area. There isn't much interest in mapping the sandy bottom so that unfocused processing would be adequate. The replica correlator performing the azimuth processing could have a threshold set within it so that several consecutive weak returns along the along-track correlation (or azimuth correlation or compression) would cause the processor to default to unfocused. If there were several strong consecutive returns however, the processor could switch to focused processing for that single point. 
A similar higher-threshold could be set for the spotlight processor (as long as the sonar had several elements and could be steered to affect spotlight imaging).

To compare, an unusual processing method has been used in NDT, where there are almost no targets (flaws; See Moshfeghi e.g. [1986] ). In the NDT case however, the processing is simpler (a shift-add technique for examp!e) and the echoes are often passed through a shaping filter. The similarity between the NDT synthetic array processing and the one described here is that the environment is sufficiently free of many targets, and therefore the processing scheme may be altered for speed and computation purposes.

\subsubsection{3-D Image Processing}

The traditional implementation of SAR, and the few experiments in SAS have usually had a sonar platform traveling along a straightline path. This creates a linear aperture, over which the echo returns are synthesized and allows the formation of a 2-dimensional (2-D) image of target reflectivity; one dimension is the $y$-axis or along-track dimension, and the other dimension is the slant range. A I-D synthetic aperture thus gives a 2-D image. Elevation may not be found from these synthetic apertures because they lack any vertical baseline. One approach to resolving elevation, and thus changing slant-range imaging into groundrange/elevation imaging is by having two real artennas (antennae) on the platform which are spaced a known, fixed, vertical distance apart. An interferometric image may be synthesized which gives an estimate on elevation, and therefore gives an approximation to 3-D imaging. Another approach to 3-D imaging using synthetic apertures is by making two separate fly-bys of the target area along two different, but parallel, flight paths. Separate images are then created for these fly-bys, and then taken together form a stereo pair (see Elachi [1988] for examples].

An alternate approach involves flying the platform along a path within a billboard, which then gives a 2-D synthetic array: 1-D along $y$, and 1-D along $z$ (elevation). A 2-D aperture therefore gives 3-D imaging capability. This is shown in Figure 2.33. This will, of course, complicate the processing and require navigation accuracy to identify the actual flight path, so that good coherent images may be formed.

See Castella [1971] for 3-D sonar imaging and Graham [1974] for interferometric SAR. 


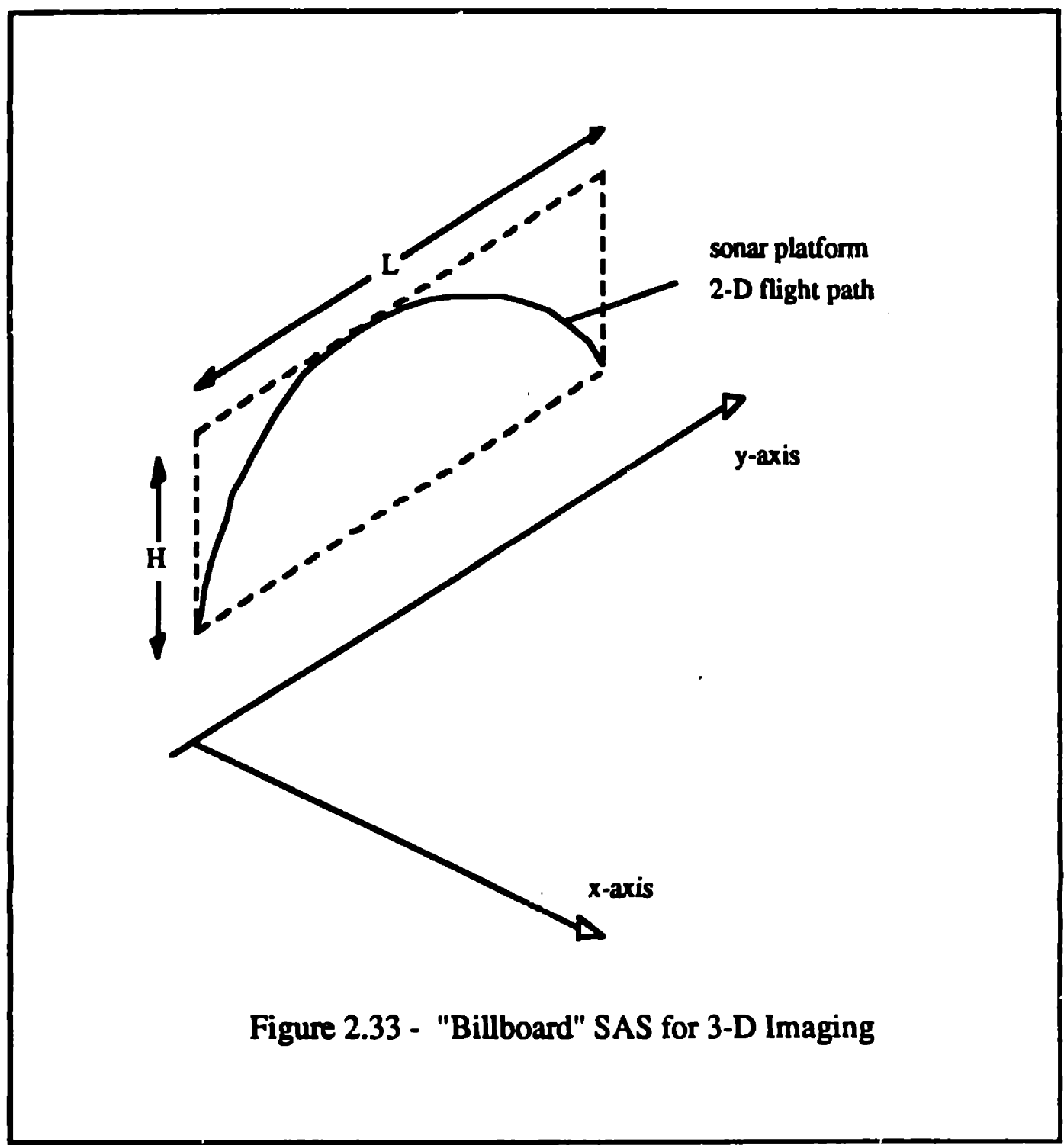




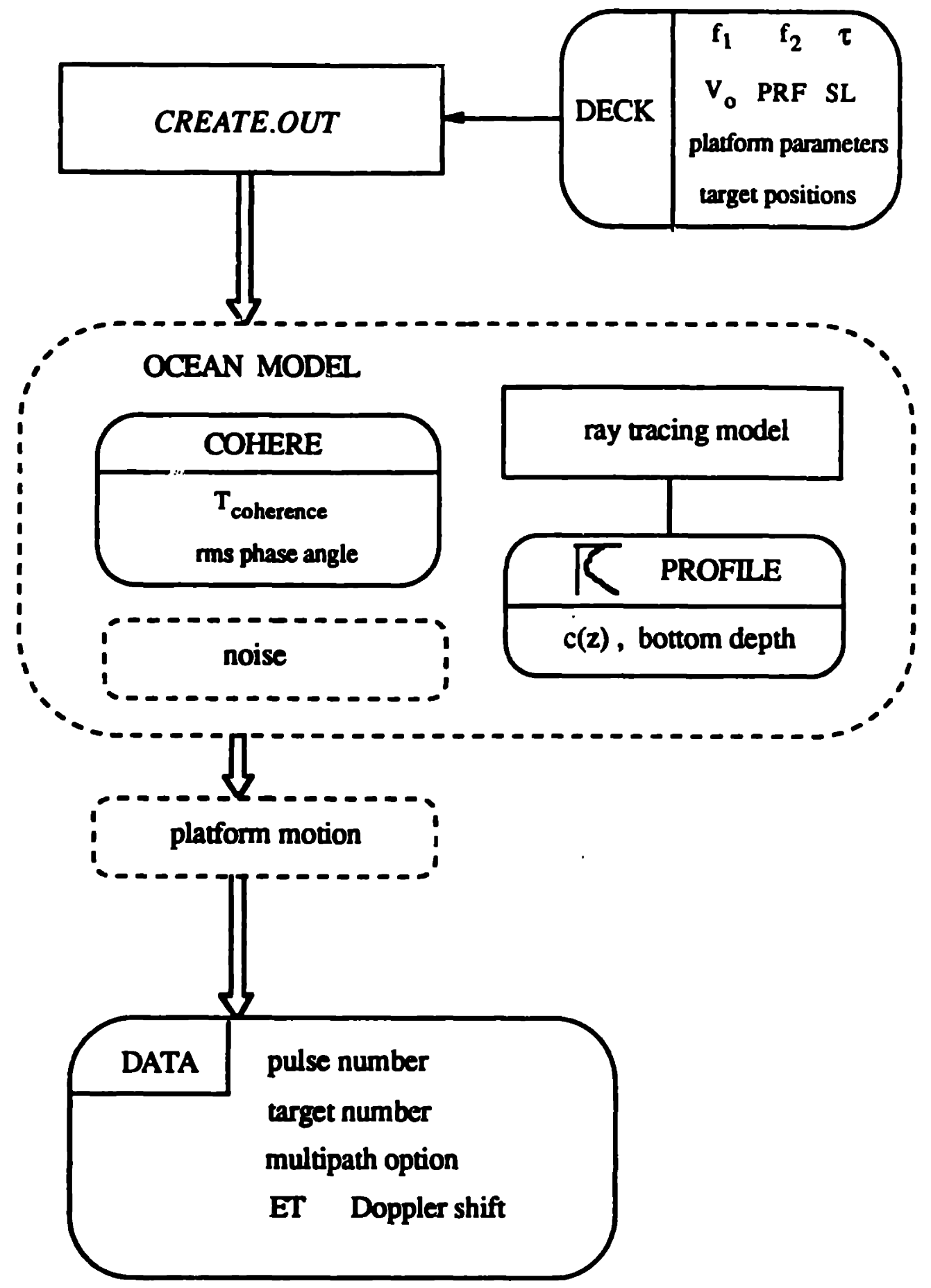

Figure 3.1 - Flowchart for Echo Data Creation 


\section{Chapter 3}

\section{Processing Methods and SAS-OCean Computer Model}

This chapter concerns the processing methods and the computer modeling used to simulate the ocean environment, the sonar and its platform and platform motion, and the sound propagation for synthetic aperture sonar imagery. The limits on the use of this computer model are also discussed, including platform speed, pulse duration, pulse bandwidth, and the number of targets.

\subsection{Computer Program Architecture and Processing Methods}

The model for simulating synthetic aperture sonar imagery for this thesis is divided into two separate computer programs. The first, create.out, creates symbolic sonar data. The second program, synth.out, takes the symbolic sonar data, creates segments of echo time series with the proper Doppler shifts, noise, sidelobe influences, surface and bottom reflections, and medium coherence time, and processes the time series into two-dimensional (along-track vs. slant-range) images of the ocean bottom reflectivity. The program create.out is described first.

\subsection{1 create.out}

A real SAS system would pulse and then listen for echoes. The listening step would be in the form of a recording, or the recording of a set of echo time series. A new time series would be created for each sequential pulse the sonar made as it transits along its path. However, full time series records for (often) large numbers of pulses would take commensurately large ASCII or binary storage in a computer memory or on a storage drive. To avoid potential storage problems on the computers used to run the simulations in this thesis (a Digital Equipment Corp. MicroVax II, and an ALLIANT FX/40), only key symbolic data are taken and recorded. These symbolic data include the pulse number, the elapsed time (or ET, the round-trip time of flight) to a target, the target number, and the Doppler shift (the Doppler formulation for a moving sonar and a fixed target is shown in Appendix D). Multiple ET's are recorded for simulations having multiple targets, and likewise additional ET's are recorded for multipath propagation. Each ET is calculated for ray-path travel through a refracting environment, which is read from the file PROFILE . Each ET is also calculated for the round-trip time from a moving sonar platform, so that the out-travel time; is not (usually) the same as the back-travel time. For each ET calculated, an accompanying Doppler shift 
and normalized $B_{x m i t}(\psi, \beta)$ and $B_{\operatorname{recv}}(\psi, \beta)$ for the out-and-back beampattern weights are also calculated, where $\psi$ is the (depression) angle measured from the horizontal, and $\beta$ is the angle measured in the fore-aft direction from the broadside direction. These data are written to an output file called DATA. By having values of elapsed-time, Doppler shift, and beampattern weight, the full time series echoes for each pulse, including all targets and all medium effects, may be reconstructed one at a time, so that computer storage is minimized. The accumulation of these pieces of symbolic data for each pulse/target/multipath represent the symbolic creation of Doppler shifted echoes from targets for the duration of the sonar platform motion. Even though no time series are created by this program, they are symbolically stored to be later created into a time series. These steps are schematically represented in Figure 3.1. The maximum number of pulses is 400 due to the size of the computer storage arrays in the program synth.out.

The program create.out reads from a control file called DECK, which has parameters such as frequencies, pulse duration, platform speed, and target locations. Annotated examples of DECK, PROFILE, and portions of DATA are shown in Appendix C. Table 3.1 summarizes the limits of create.out .

\subsection{2 synth.out}

These symbolic pieces of data are transformed, one at a time, into segments of time series in the second program synth.out. This program performs the necessary range and azimuth compression on the ET, beam pattern, and Doppler numbers in DATA, into a two-dimensional map (along-track and slantrange) of target reflectivity. This program is controlled by a file called SAS_CONTROL which has the type of imaging (focused or unfocused), and the area location to be imaged in along-track, slant-range coordinates. The number of image points and the spacing of points within the image area is automatically determined by the transmit signal bandwidth and by the sonar's horizontal dimension. When a certain point is imaged, say at slant-range/along-track coordinates $x_{0}, y_{0}$, synth.out creates the segments of time series for a target which would be located at the position $\mathrm{x}_{0}, \mathrm{y}_{0}$, whether there were a target there or not. What this means is that all other targets on the bottom (at different locations), but at the similar slant ranges to the sonar, will have echoes which would fall within the segment of time series being created because they were in the view of the sonar mainlobe and sonar sidelobes. Once the segment of time series is created (including the possible echoes from all other targets, as influenced by the sonar beam pattern to each target), a replica correlation is performed between the measured time series segment, and the postulated echo which would have been returned from a target at position $x_{0}, y_{0}$. This sequence of creating a time series segment and performing a replica correlation on it, is done for each pulse of the sonar when the image point $x_{0}, y_{0}$ is within the illumination of the $-3 \mathrm{~dB}$ mainlobe of the sonar. The length of the time record in the segment of the time series is determined by the pulse duration used in the simulation. Amplitude 
weighting may also be performed at this step on the replica, which is a means to lower the range sidelobes at the expense of a wider mainlobe. This replica correlation in the range direction is called pulse compression, and is also called range compression. Once the replica correlation has been done for the time series segment, the value of the correlation is stored in a buffer array.

\section{Table 3.1 - Program Capabilities/Limits}

\section{Ocean and Targets:}

10 possible point targets located in $x, y, x$

10-point refraction sound speed profile, $c(z)$

bot'om depth (constant)

coherence time (in seconds) and $\mathrm{ms}$ phase angle bound for coherence time

3 possible multipaths: direct-path (DP), surface-reflected (SR), and bottom

surface reflected (BSR)

frequency dependent absorption

surface and bottom irregularities (introduces a random scatter phase and reflection loss)

random noise and SNR capability

Platform:

single sonar transceiver (rectangular $\mathrm{D} \times \mathrm{H}$; or circular $\mathrm{R}$ )

sonar depression angle from horizontal (but no squint angle)

on-axis source level (re $1 \mu \mathrm{Pa} @ 1 \mathrm{~m}$ ), PRF, $\mathrm{f}_{1}, \mathrm{f}_{2}$

pulse duration $\tau \leq 1500 / f_{\text {high }}$

up to 400 pulses

transmit pulse envelopes: cos, triangle, rectangle

Processing:
focused or unfocused
limit the size of the aperture length $L^{\prime}$ processed $\left(L^{\prime}<L\right)$
replica waveform: transmit pulse, or Doppler shaped replica pulse
additional along-track (azimuth) weighting/taper. rectangle or Hanning
sound speed estimated profile

The next sonar pulse is chosen, and a new time series segment is created for the appropriate location which intercepts the hyperbolic range migration path for $x_{0}, y_{0}$ and then replica correlated, and the value of the correlation is also stored in the buffer array. This is repeated for as many sonar pulses as the target 
point $\mathrm{x}_{0}, \mathrm{y}_{\mathrm{o}}$ was within the illumination of the sonar mainlobe. Only the $-3 \mathrm{~dB}$ mainlobe data is used because only the mainlobe has sufficiently large, useful echoes. Echoes from sidelobes could, in principle, be used but for the purposes of this thesis they are not. The array of values in the buffer represent the range-compressed target reflectivity for image point $x_{0}, y_{0}$ as taken from each sonar-pulse platform position, as the platform moved along a y-direction path. This array of values is an azimuthal array (or along-track array). We may now perform an along-track replica correlation on this array, and also include any amplitude weighting to reduce along-track (or azimuthal) sidelobes. The result of these replica correlations is a complete range- and azimuth-compressed target reflectivity for image point $x_{0}, y_{0}$. The remaining task is to take the magnitude of the target reflectivity (i.e. the absolute value), and write the magnitude and the $\mathrm{x}_{0}, \mathrm{y}_{\mathrm{o}}$ coordinates into an output file called IMAGE. This completes the imaging of the point, and now the next point inay be imaged by the same procedure. These steps are schematically shown in Figure 3.2.

An example of SAS_CONTROL and a partial example from an IMAGE file are shown in Appendix C. Table 3.1 summarizes the options for syni,h.out.

\subsection{Platform/Sonar Capabilities and Assumptions}

The platform used in the computer model is assumed to travel at a known constant speed $\mathrm{V}_{0}$ along a known, straight, horizontal path. The straight path may be perturbed from the straightline path by adding an error to each ET. It is assumed that the dominant sources of motion which influence SAS images are from platform motion that is horizontal (since the SAS is a side-looking sonar). The amplitude and frequency of this motion is variable, and the motion may also be random and have a suitable amplitude scale.

The sonar looks to one side in an $x, y$ plane, and may have a depression (negative value) or elevation (positive value) angle from the horizontal. The sonar transducer is a single element, and may be either a rectangular or circular piston. The sonar is used for both transmit and receive (it acts as both a projector or transmitter, and acts as a hydrophone). The image area is assumed to be in the far field of the sonar, and so the sonar beam pattern is calculable from a sinc function (see Appendix A). The out- and back-direction beam pattern weights are also determined for each ray path used from the moving sonar to any target. In this way, the influence of transducer sidelobes may be studied. The transmit frequencies are always in the form of an up-chirp, starting at frequency $f_{l o w}$ and ending at frequency $f_{\text {high }}$ in $\mathrm{Hz}$. A narrowband (single frequency) signal may be transmitted by using closely spaced values for $f_{l o w}$ and $f_{\text {high }}$. The transmit signal may also be tapered within a cosine envelope, within a triangle envelope, or have a rectangular envelope. 


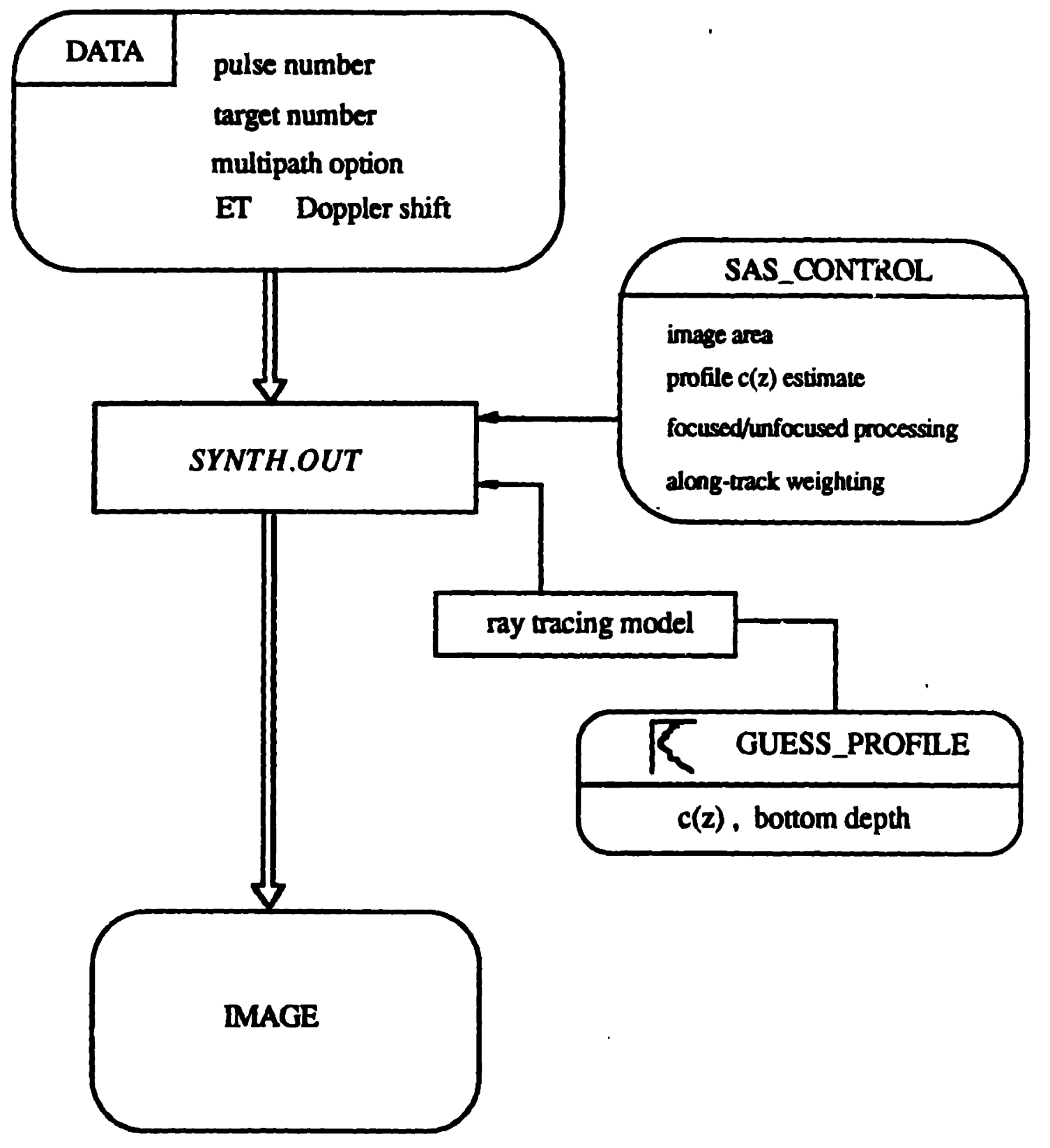

Figure 3.2 - Flowchant for Image Synthesis from Echo Data 
The pulse duration $\tau$ in the synth.out model is restricted by the computer array size of a target echo by the following:

$$
\tau \leq \frac{1500}{f_{\text {high }}}
$$

This comes from the fact that the array size was chosen to be 15,000 points, and the waveform sampling was set at 10-times the highest transmit frequency.

\subsection{Target, Ocean and Boundary Properties and Assumptions}

The targets used in the SAS model may be viewed as rigid spheres or as comer reflectors. There may be as many as 10 targets, and they are usually placed on the ocean bottom. It is not a requirement that they are bottom-mounted, but a midwater target will not be imaged in three dimensions for the usual SAS imagery, since synthetic arrays are usually extended in one direction, and therefore may only image in two dimensions (along-track, and cross-track). A two-dimensional synthetic aperture would be necessary to image targets in three dimensions (see section 2.5.3). The target strengths and target locations are entered into the file $D E C K$, and the targets are assumed to have target strengths that are constant for any angle of sound illumination.

The ocean is modeled with horizontal stratification, and is given a sound speed profile in the file PROFILE. This file has 10 data point sets and has an eleventh entry which is the bottom depth. At present, the surface and bottom boundaries in the computer model are horizontal. Absorption at these boundaries is approximately modeled by introducing amplitude weights (i.e. reflection coefficient $<1.0$ ), and scattering from surface or bottom roughness is approximately modeled by perturbations in elapsed-time (ET) from pulse to pulse. A background noise level may be introduced having a signal-to-noise ratio as referenced to the closest target echo. Sound propagation is modeled with ray-tracing through an absorptive refracting environment. Three possible rays are used: the direct path (sonar to target and back), a surfacereflected path (sonar to surface to target, and back the same way), and a bottom-surface path (sonar to bottom to surface to target, and back). The direct path may be used alone or in combination with the other two. Absorption along ray paths is approximated by a frequency-dependent equation (see Thorp [1967] and Urick [1982] ). The effects of the surface and bottom absorption and roughness may be altered by using different coefficients. These boundary parameters are found in the file SAS_CONTROL. 
The coherence time of the ocean medium is artificially introduced by adding an error term to the elapsed times (ETs). The coherence time and rms phase (in degrees) is entered into a file called COHERE , which is read by synth.out. When a location is being imaged, synth.out adds a random error term to each ET except the first pulse which illuminated the point (via the mainlobe). The first pulse used in the image formation has no error because it is the reference the following pulses are compared against. The error added to each ET is made smaller than the rms phase limit when the clocktime (beyond the first pulse) is less than the coherence time, but the error added is made larger than the rms phase limit otherwise. This means that the sonar has a time limit (i.e. the dwell time), and a distance limit over which it travels before the echoes from a single target become incoherent (the phase error is too great). This fixes the maximum useful length of a synthetic array which may be formed an 1 therefore places some limits on resolution in the along-track (or azimuth) direction.

\subsection{Processing Described Mathematically}

This section develops the mathematics of the processing which have been hitherto ignored. For the sake of keeping the equations as simple as possible, we ignore geometric spreading and absorption. We consider the reflections from a single point target on an otherwise smooth bottom. We also use the slantrange and along-track 2-D environment.

Using the customary notation of this thesis, we have a platform traveling along the $y$-axis at constant speed $V_{0}$. The target is located at $x_{0}, y_{0}$. The platform transmits an FM chirp pulse of duration $\tau$ every $T$ seconds, and the $P R F=1 / T$. The interpulse distance $D_{\text {pulse }}=V_{0} / P R F$. We plan to transmit a total of $N$ pulses as we travel past the target location. We also assume, for now, that we plan to image a single point (or image pixel).

The $n$-th transmit pulse is in the form

$$
\begin{aligned}
& \qquad s_{n}(t)=A \sin [2 \pi f(t) t] \cdot \operatorname{rect}\left[\frac{t}{\tau}\right] \\
& \text { where } A=\text { amplitude } \\
& \operatorname{rect}[t / \tau]=1 \text { for } 0<t<\tau \text {, and zero elsewhere } \\
& f(t)=f_{1}+a / t \\
& a=\left(f_{2}-f_{1}\right) / \tau \text {, the chirp rate }
\end{aligned}
$$

and $f_{1}$ and $f_{2}$ are the lower and upper frequencies. 


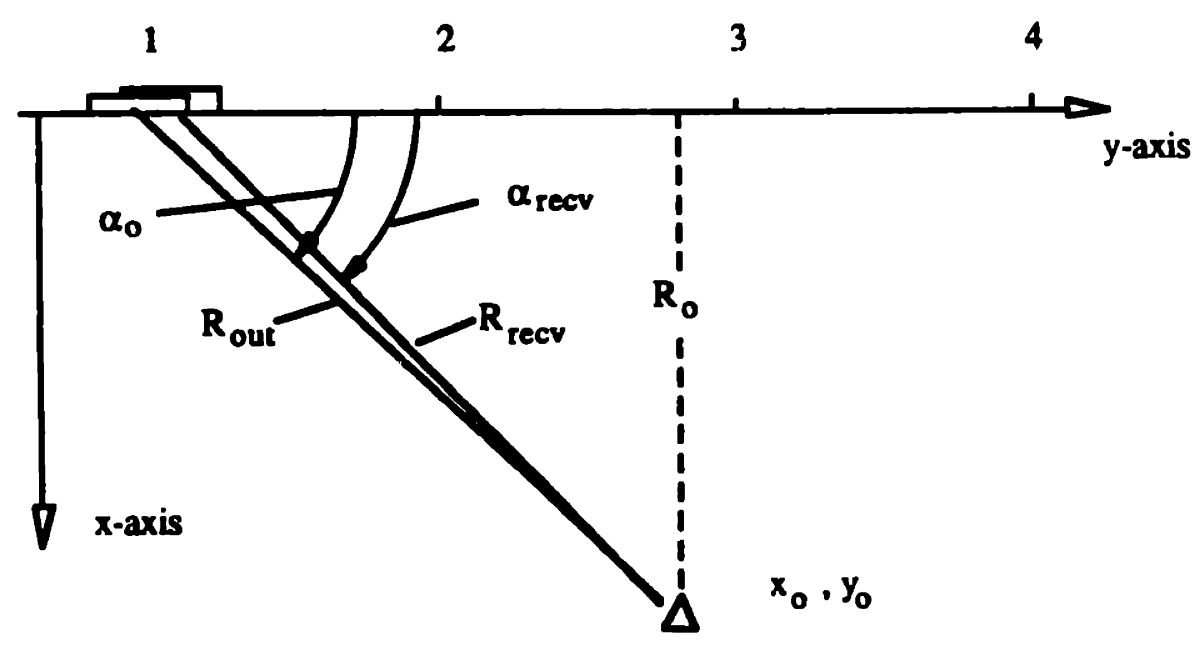

Figure 3.3 - Imaging Geomtery, 2-D example

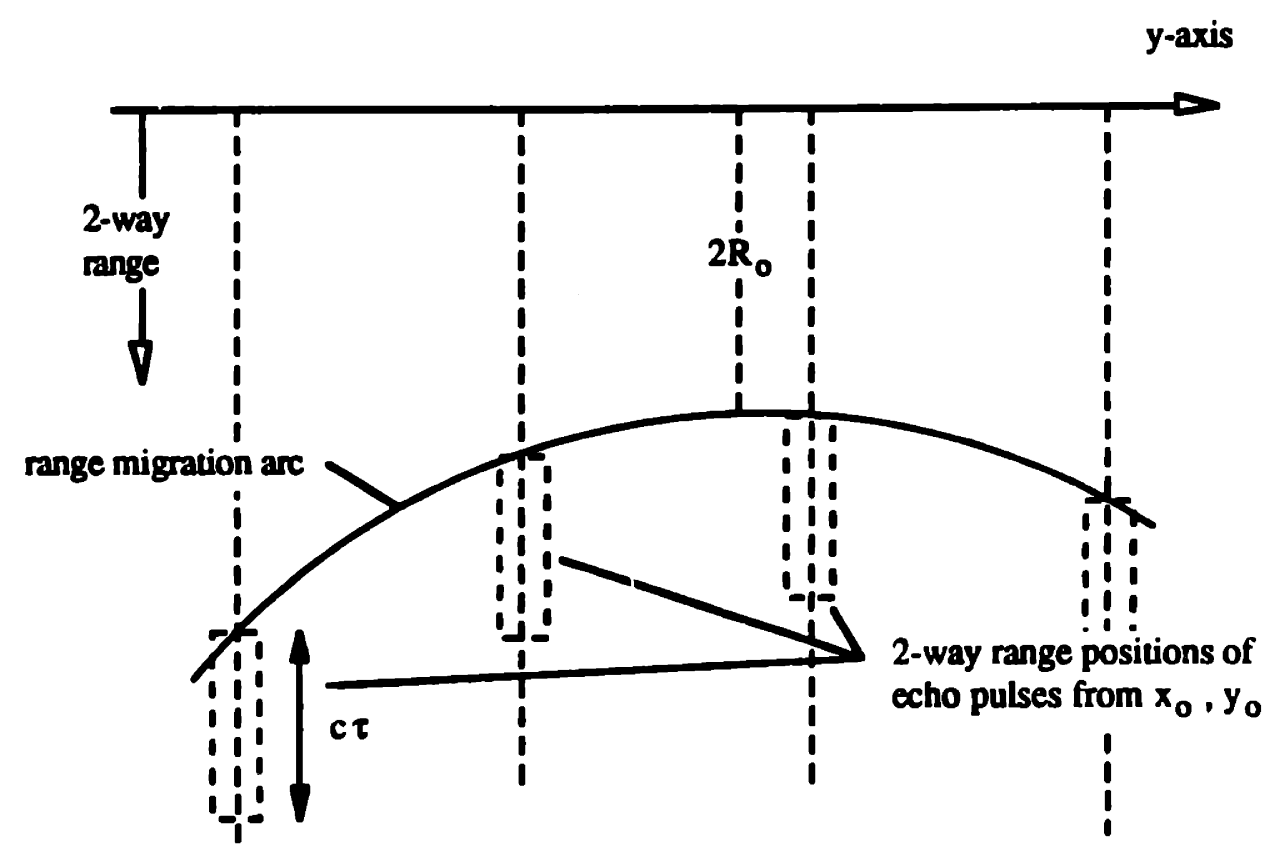

Figure 3.4 - 2-Way Range Migration for $x_{\circ} y_{0}$ 
This signal leaves the moving sonar, strikes the fixed target, and echoes back to the moving sonar. The received signal is in the form of

$$
r_{n}(t)=\hat{A} \sigma \beta^{2} \sin \left[2 \pi f(t) D_{f}\left(t-\frac{R_{\text {out }}+R_{r e c v}}{c}\right)\right] \cdot \operatorname{rect}\left[\frac{t}{\tau}-\frac{R_{\text {out }}+R_{\text {recv }}}{c \tau}\right],
$$

$$
\begin{aligned}
& \text { where } \widehat{\mathbf{A}}=\text { reduced amplitude } \mathbf{A} \text { (including geometric spreading and absorption) } \\
& \sigma=\text { the target reflectivity (which we assume constant with frequency and incident angle) } \\
& \beta^{2}=\text { the combined out-back beampattern function which is frequency dependent } \\
& D_{\mathrm{f}}=\text { the Doppler factor }=\left\{1+\left(\mathrm{V}_{\mathrm{d}} / \mathrm{c}\right) \cos \left(\alpha_{0}\right)\right\}\left\{1+\left(\mathrm{V}_{\mathrm{o}} / \mathrm{c}\right) \cos \left(\alpha_{\mathrm{recv}}\right)\right\} \text {. } \\
& \text { See Appendix D for the derivation of the Doppler factor. } \\
& c=\text { the sound speed (assumed constant for purposes of simple equations) } \\
& \tau=\text { the pulse time duration }
\end{aligned}
$$

and $\mathbf{R}_{\text {out }}$ and $\mathbf{R}_{\mathrm{recv}}$ are the respective out- and back-direction distances from sonar to target.

The geometry for the $n$-th pulse is shown in Figure 3.3.

Though the area we are mapping is unknown, we assume that the area (i.e. the sea bed) is immobile and that we know the direction and speed of our sonar platform. Since our intention is to image the point $x_{0}, Y_{0}$, we can sketch a range migration for the point as it passes the view of the sonar. This is shown in Figure 3.4. Note the rectangular box along the range-axis for the $n=1$ pulse. This box represents where the $\tau$-length (range $=c \tau$ ) echo for the point $x_{0}, y_{0}$ is found. We "grab" the time series within the box and correlate it with a replica of the transmitted signal. This is known as cross correlation (or replica correlation) and it is a range compression step (see Appendix B). The correlation for a single point such as $x_{0}, y_{0}$ is a multiply-sum step, which we denote as $r c_{n}$ (range-compressed, $n$ ) and write as

$$
r c_{n}\left(x_{0}, y_{0}\right)=\int_{\frac{2 R_{n}}{c}}^{\frac{2 R_{n}}{c}+\tau} r_{n}(t) \hat{s}(t) d t
$$

$$
\text { where } \begin{aligned}
\hat{\mathbf{s}}(\mathrm{t}) & =\text { the transmit replica, or } \\
& =\mathbf{a} \text { Doppler shifted transmit replica } \\
2 \mathbf{R}_{\mathbf{n}} & =\mathbf{R}_{\text {out }}+\mathbf{R}_{\text {back }} \text { for the } \mathbf{n} \text {-th pulse }
\end{aligned}
$$

Note that a Doppler shifted replica is included here as an option. The form of synthetic aperture processing used in this thesis includes the feature that the replica used in the correlator is a better match 
when the Doppler shift (via the Doppler factor) is included in the replica waveform. This feature has a small effect for cases where the platform moves with a small velocity, and no effect for the case of stop-andgo data acquisition (as in the ground penetrating radar described in section 2.1.2 where there is no Doppler shift because the platform has no motion during the transmit/receive operation..

The result $\operatorname{rcn}\left(x_{0}, y_{0}\right)$ in equation 3.4 is a single number, either positive or negative, which represents the value of the replica correlation (or pulse compression) for the $n$-th pulse at the location $x_{0}, y_{0}$. We then repeat the step of pulse compression for the entire set of $\mathbf{N}$ pulses which then gives us an $\mathrm{N}$-set of alongtrack (or azimuthal) correlation values.

If there were indeed a target at the location $x_{0}, y_{0}$ then the members of the $\mathrm{N}$-set array described above would all have the same sign (either positive or negative). If we summed these numbers together, they would add constructively. If we summed them and then took the magnitude (the absolute value), we would find the strength of the image at the location $\mathrm{x}_{0}, \mathrm{y}_{0}$. Conversely, if there was no target at the location $x_{0}, y_{0}$ but was instead at a nearby location, we would find that the along-track $\mathrm{N}$-set array would have some positive and some negative values. The sum of these numbers would be somewhat destructive, and yield a smaller magnitude image result as it should because there was no target present at the location $\mathrm{x}_{0}, \mathrm{y}_{0}$. The summation process is an along-track compression (or azimuth compression), and it is really a focusing step. We write this as

$$
\operatorname{image}\left(\mathrm{x}_{0}, \mathrm{y}_{0}\right)=\left|\sum_{1}^{\mathrm{N}} \mathrm{rc}_{\mathrm{n}}\left(\mathrm{x}_{0}, \mathrm{y}_{0}\right) \mathrm{q}_{\mathrm{n}} \Delta \mathrm{y}\right|
$$

where $\quad \mathrm{N}=$ the number of pulses used to image the location $\mathrm{x}_{0}, \mathrm{y}_{0}$

$q_{n}=$ the along-track (azimuth) replica; constant 1.0 for rectangle taper, variable for Hanning, cosine, or other taper.

$\Delta y=$ the interpulse distance $D_{\text {pulse }}=V_{0} / P R F$.

We also have the ability to taper (or weight) in both the range direction and in the azimuth direction by applying the taper to the: time series in each range box for range tapering, or to the $\mathrm{N}$-set azimuth array of range compression numbers for azimuth tapering. In either case, the weighting is intended to reduce the sidelobe levels at the expense of a wider mainlobe. 


\subsection{A Note on Processing}

The processing method shown in section 3.4 performs exact focusing at all points in the image plane either by true focused processing (using an exact match for the range migration of each image point) or by unfocused processing (where the range migration is ignored). These are effectively geometric correlators or matched filters. The use of an image point Doppler-shifted waveform in the replica correlator completes the other half of the match filtering process. The combined use of the Doppler-shifted correlator, and the range migration correlator gives the best theoretical match of the postulated target response to the actual target response.

The processing used in SAR however, is usually different from the method shown here. Because there are many processing schemes (or image mechanizations) used in SAR, and several schemes of overcoming deficiencies in these methods, they will not be covered here. Instead the reader is urged to review the notion of "focus at all points" shown in Chapter 2, and to review pulse compression for fixed targets from moving platforms and the consequences of using up-, down-, and no-Doppler-shifted replicas in the pulse compression. After these ideas are ingrained, the reader may tackle the processing schemes described in Elachi [1988], and in the other SAR references listed in the bibliography. In short, the processing shown in this thesis takes no short-cuts, is exact, but has the deficiency of being computationally intensive. The various SAR processing schemes take one or more short-cuts, but still give sharp images and with reduced computational effort. 

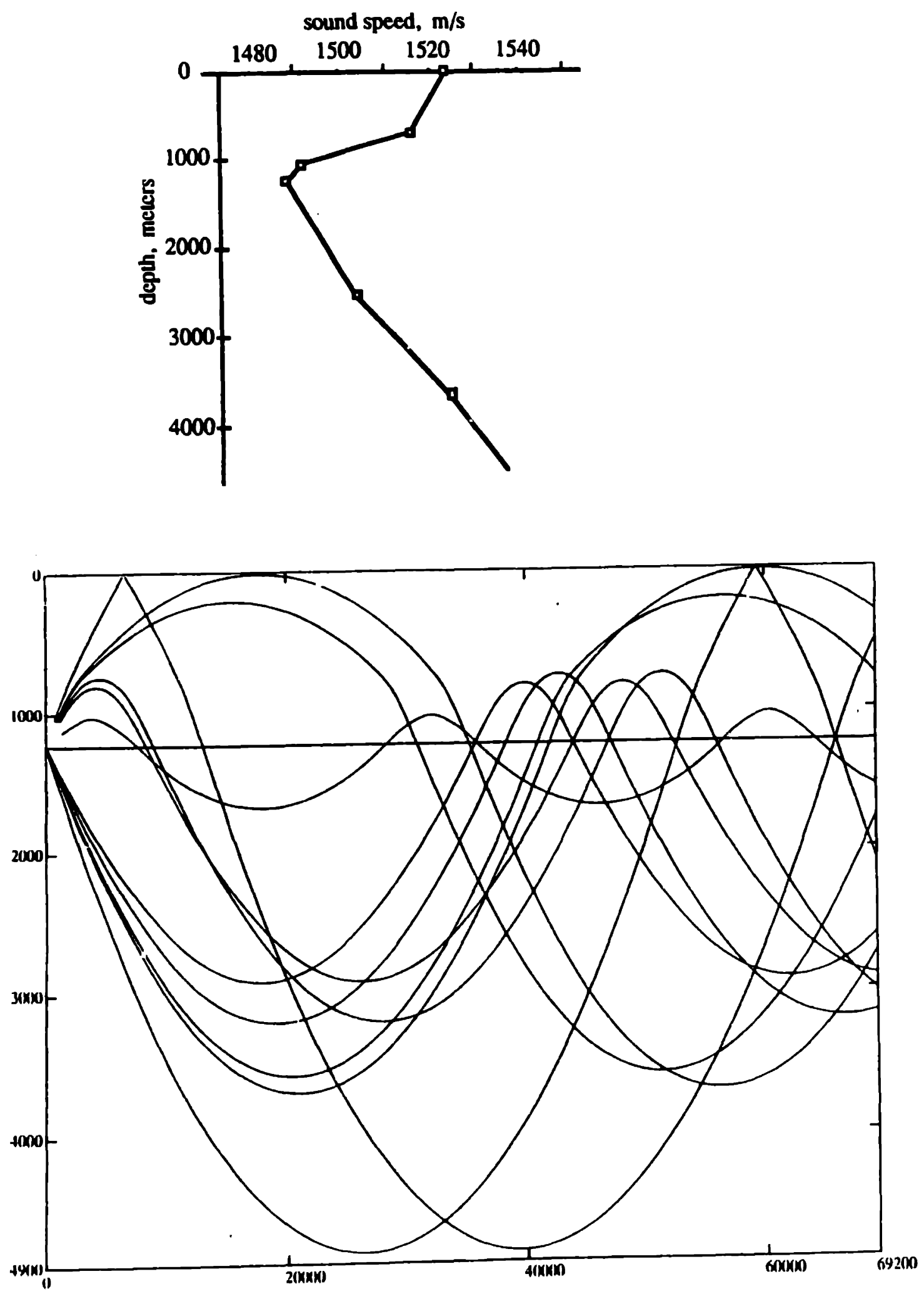

Figure 4.1 - Ray Trace Example, compare with Urick [1982], p. 7-2. 


\section{Chapter 4}

\section{Environmental and Platform Influences on SaS}

This chapter discusses the influence, of the environment (usually the ocean) and the sonar platform on the performance of a synthetic aperture sonar. The chapter is divided into three parts; multipath propagation. medium stability, and platform motion.

\subsection{Multipath and Refraction Propagation}

Because the water environment in which a sonar operates, whether it is a lake, a river, or an ocean, is predominantly stratified in the vertical dimension (in horizontal layers), and because the water surface (an air-water interface which is almost a perfect reflector) and the bottom (the sea bed e.g.) act as reflecting boundaries, the propagation of sound from a source to a target (or to a receiver) is almost always contaminated by multipaths, and is often accompanied by refraction. An exception to this is the case where both the sound source/receiver is highly directional, and the path distance is very small compared to the distance to the surface and bottom boundaries.

Analysis of the water environment (often called the "water column") which supports acoustic waves shows that pressure, temperature and salinity have domiriant influences on the propagation speed (i.e. the sound speed). Further analysis shows that acoustic wave propagation may be viewed from a ray-approach, where the sound rays bend as they travel due to the refraction of sound caused by local changes in the sound speed. In this way, sound is refracted in exactly the same way as light may be refracted in a prism. One simple approach for solving a sound propagation problem in a horizontally stratified ocean is to take the sound-speed versus depth, (i.e. the sound speed profile) and to discretize it into piecewise linear steps. This is a useful approach because it may be shown [Urick, 1982] that a sound ray travels in a circular arc if it travels in an acoustic medium having a (locally) linear sound speed profile; stated somewhat differently, the sound speed is isogradient (constant slope). A complete ray trace (the entire ray path) may then be found from a source to a distant location by connecting a collection of piecewise circular arcs. Computer codes have been written to do such a task, and they are found to be efficient and fast.

A feature of propagation in a stratified medium is that the waves often travel from a source to a distant location in multiple paths. An example of this multiple path, or multipath, phenomenon is shown in Figure 4.1 as plotted in a range versus depth coordinate system. 


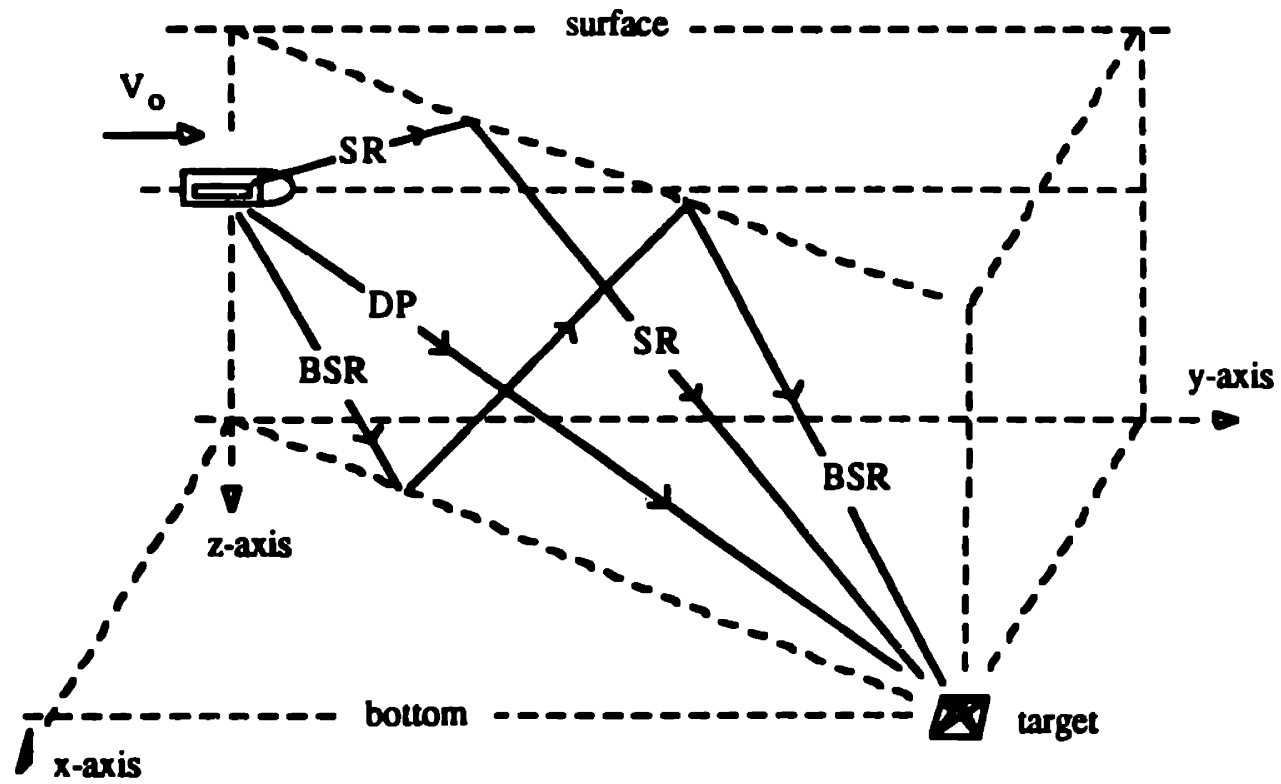

A. Multipath in an Isospeed Ocean

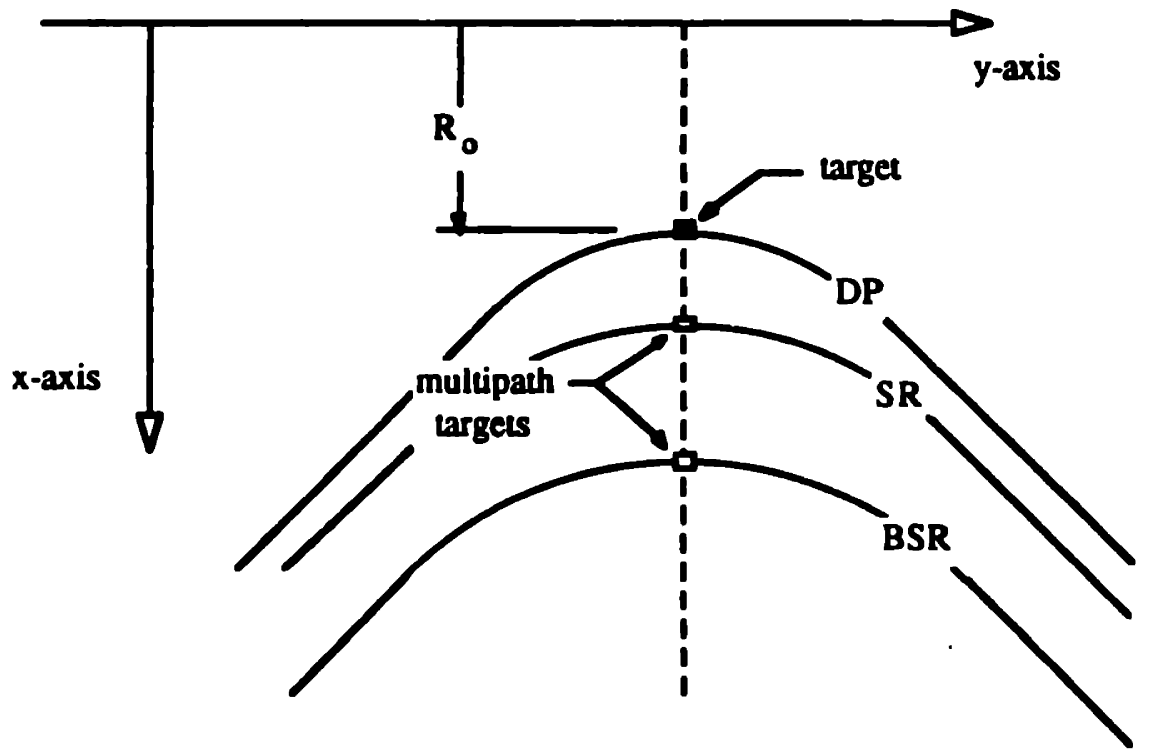

B. Range Migration

for Isospeed Multipath

Figure 4.2 - Multipath Propagation 
A source is located in the sound channel (the sound speed profile minimum) at a depth of 1240 meters, the bottom is at $\mathbf{4 9 5 0}$ meters, and the sound speed profiie for the example is shown at the top of the Figure. A number of ray paths have been traced, and there are many places where these rays cross one another. The ray crossing positions show that there are multipath rays which connect the crossing position to the source position. The rays in this figure were generated from the ray-tracing code used in the computer models described in Chapter 3, and the figure may be compared with Urick [Figure 1, p. 7-2, 1982].

Note also that, even in cases where the sound speed profile is constant (or isospeed), multipath from source to a target location may still exist due to reflections from the surface and from the bottom.

Computer models for synthetic aperture radar (SAR) and the few synthetic aperture sonar computer models known to exist usually assume either isospeed or isogradient propagation, without refraction, without reflecting boundaries (like the ocean surface and ocean bottom) and without multipath. These assumptions have not been made in this thesis. The computer model described in the previous chapter has a refracting environment which enables multipath, and it has reflecting surface and bottom boundaries which also create multipaths. What remains to be shown is what the effects of the multipath are on the formation of synthetic aperture images. Two simple cases will be shown to illustrate the effects of multipath on synthetic aperture imaging. The first is an isospeed case to show the multipath effects of the ocean surface and bottom, and the second is a deep water case with a sound speed profile. Though refraction and multipath are phenomena found in radar propagation, they do not greatly hinder the performance of a SAR and are seldom mentioned in the SAR literature.

\subsubsection{Isospeed Case - Surface and Bottom Erfects}

Consider the geometry shown in Figure 4.2A. For the moment we allow the surface and the bottom to act as perfect horizontal reflectors. Three distinct ray paths, or multipaths, are identified which connect the source on the sonar platform to thi target: DP for direct, SR for surface reflected, and BSR for bottomsurface reflected. In this thesis we assume that the acoustic ray paths return from a target in the same fashion as they travelled out to the target. Thus a direct path out to a target yields a direct path back from a target; a surface reflection from source to target has an echo which returns along a surface reflection path, and similarly the bottom-surface path from source to target gives a surface-bottom return path. A basic assumption is then implied that the target reflects sound energy back in the same direction it received it. This is never entirely true for real targets in the ocean, even for spheres and corner reflectors. However, a more sophisticated scautering model enormously complicates the processing because scattering, by itself, is a complicated problem worthy of separate study. Therefore, the scattering model used in this thesis is 
admittedly a simple one, but is reasonable in order to show the imaging performance of a synthetic aperture sonar.

A transmitted pulse from a moving sonar such as the one shown in Figure 4.2A will result in three returned echoes, which occur at elapsed times corresponding to both the round-trip and to the sound speed along each path. Since we have simplified this example to an isospeed case, the sound speed is constant for any range and depth and all rays appear straight. In terms of the range migtation of the target, we would find that there are ithree migration paths corresponding to the three perceived echoes; these are labeled (again) DP, SR, and BSR in Figure 4.2B. Upon forming a synthetic aperture along the sonar path, the image would result in three, target images at the same along-track position and at different cross-track (slant range) positions. The strength and possible overlap of the target images of course depends on the length of each of the three multipaths, and the normalized beampattern weight for each of the three different depression/elevation angles. Two of these images are 'ghost' images due to multipath, and are aliases of the true target image (but these are distinct from the alias images due to undersampling).

If we now allow the surface and bottom to be non-flat, somewhat absorptive, and time-varying, we would find that the range migrations for the SR and BSR multipaths will have time-varying displacements and time-varying amplitudes. This tends to ruin the matched filtering process of focusing, and spreads the ghost target images over an area, thus diffusing it. The multipath example shown here applies equally well to examples where the sound speed has a profile (i.e. not constant with depth). This would result in curved rays in Figure 4.2A, and corresponding displacements of the arcs in Figure 4.2B in the $\mathrm{x}$-direction.

\subsubsection{Deep Water Refraction Case}

Consider the case where the moving sonar collects echoes from a target in deep water, and where the water environment has, for example, an isogradient sound speed profile. The range migration arc for this case is similar to those shown before, but has a different curvature. As an illustration, Figure 4.3 shows two sound speed profiles, and the corresponding range migration arcs (where only the direct path arcs are shown). The rays in the isogradient case travel a greater distance but in a shorter elapsed time than the rays in the isospeed environment, and the isogradient rays travelling to the target when the platform is not at broadside must dive deeper and hence travel even faster. So the hyperbolic range migration arc for the isogradient case is somewhat distorted. The purpose for illustrating these differences is that the hyperbolic arc used in synthetic aperture sonar imaging must replicate the range migration arc that actually existed in

the ocean during data wollection; this means the sound speed profile in the sonar model must replicate (to an as yet unspecified accuracy) the sound speed profile of the ocean in order to form sharp (high target gain) 
images. Stated in another way, the sound speed profile used in image reconstruction must match the sound profile of the real ocean.

For example, in Fig. 4.3 the isogradient profile could be an actual ocean condition; if the isospeed profile were used to reconstruct the target image, the target image will tend to be blurred or be absent depending on the extent of mismatch between ocean and model properties (sound speed).

\subsection{Environment (or Medium) Stability}

Apart from the effects of propagation (reflection and refraction which lead to multipath), a more insidious influence on SAS is that of medium stability. If the ocean medium could have its sound speed profile "frozen" in all locations and for all time, the medium could be said to have perfect acoustical stability in both space (spatial stability) and time (temporal stability). Unfortunately, the ocean is not so cooperative, and the sound speed profile does change over space, and over time. The consequence of this for the ocean researcher who uses coherent acoustic propagation, either as a means of communication, data telemetry, or for imaging, is that the phase of an acoustic wave can no longer be relied upon for time spans which exceed the temporal stability of the medium, or relied upon over distances which exceed the spatial stability of the medium. Since synthetic aperture sonars rely upon phase coherency, then there is a limit upon the integration time (i.e. how many seconds needed to accumulate data to image a target), and there is a limit to the length (i.e. how many meters) over which you can collect this data. This means that the SAS has spatial and temporal bounds placed upon it. Since synthetic aperture sonars move at speed $V_{0}$, we can say that the spatial and temporal limits of the medium are linked. Since ocean parameters are predominantly stratified horizontally, and the sound speed profile is more-or-less constant for small changes in range, then the temporal stability (the time coherence) is the dominant effect. If we are given a coherence time for the medium, say 120 seconds with a variance of $45^{\circ}$, then the maximum length of a synthetic array that would have sufficiently phase coherence over the whole length to image a target would be given by

$$
L_{\text {maximum }}=V_{0} T_{\text {coherence }} \text {. }
$$

The implications of (4.1) are that the along-track resolution of a synthetic array now has additional limits to those shown in Table 2.2, and the similar triangles argument presented in section 2.1.2 is no

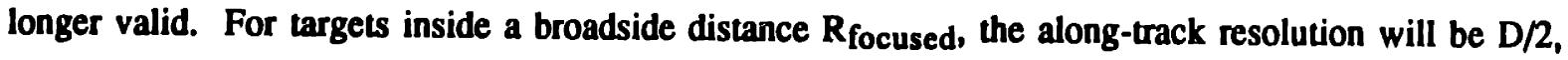
because the length of the synthetic array may be made long enough to achieve full focused resolution, or $L$ $\leq L_{\text {maximum. }}$. When the maximum length of $L$ is reached, for targets beyond $R_{\text {focused, the along-track }}$ 


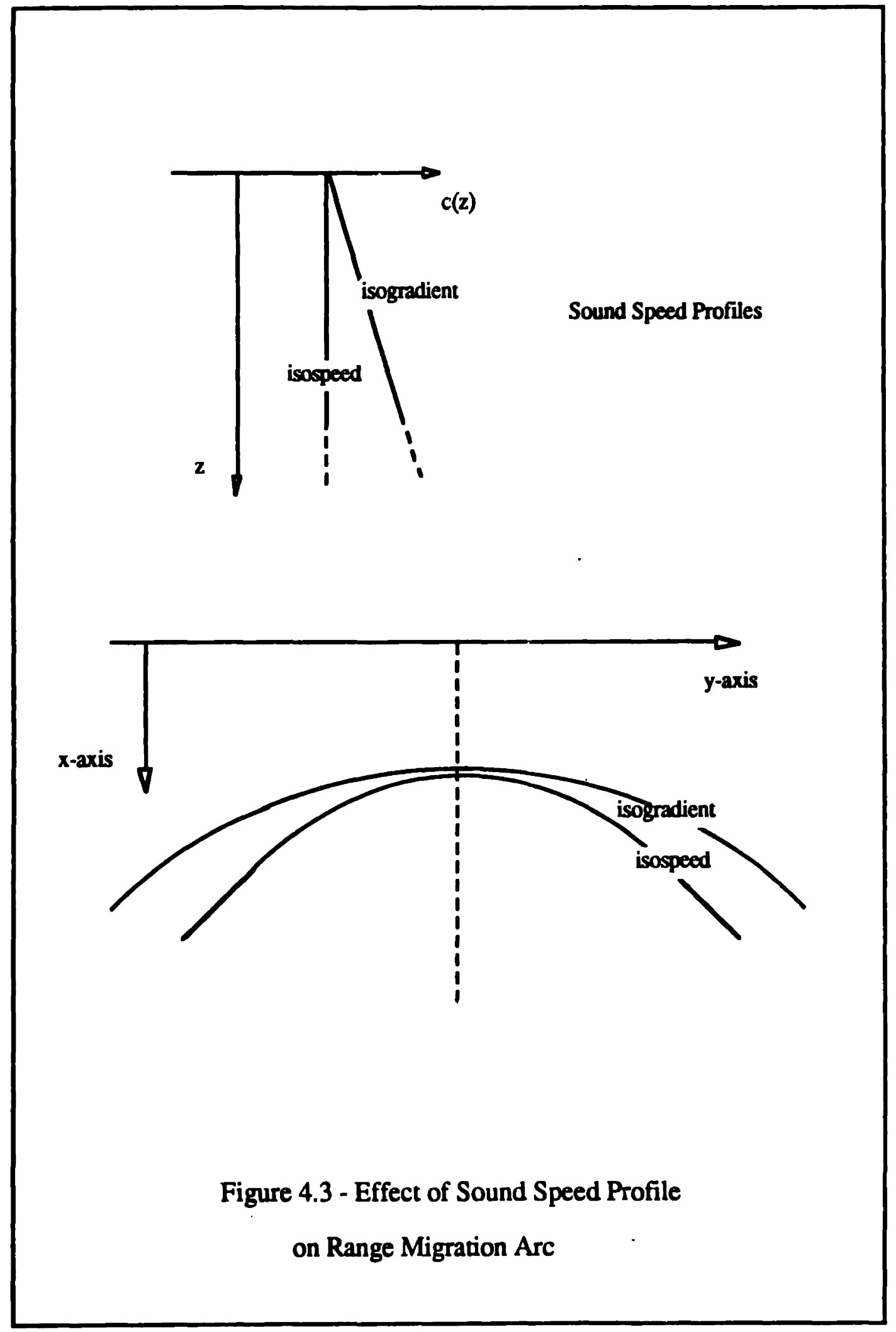


resolution will become larger and larger. This is a suboptimal focused array, where focusing is used but the array isn't as long as it should be to achieve full aiong-track resolution.

The along-track resolution may be then written as

$$
\rho_{\text {along-track }}=\frac{D}{2}+\frac{R_{0} \lambda-D_{\text {maximum }}}{2 L_{\text {maximum }}} \text {. }
$$

where $R_{0}>R_{\text {focused }}$, and $R_{\text {focused }}=\left(\lambda L_{\text {maximum }}\right) / D$.

Equation 4.2 was written in two parts to show the constant along-track resolution term (D/2), and then range dependent term (the remainder). The suboptimal, shortened-length focused array continues to have degraded along-track resolution for targets located at greater and greater broadside ranges, and eventually reaches the point where the focused array becomes an unfocused array (when $L_{\text {maximum }}=\sqrt{R_{0} \lambda}$ ).

Equation 4.2 may be rewritten as

$$
\rho_{\text {along-track }}=\frac{\mathbf{R}_{0} \lambda}{2 L_{\text {maximum }}} \text {. }
$$

where $R_{0}>R_{\text {focused }}$, and $R_{\text {focused }}=\left(\lambda \mathbf{L}_{\text {maximum }}\right) / D$,

to show the dependence on both range and wavelength. Equation 4.3 has the same form as

$$
\rho_{\text {along-track }}=\frac{R_{0} \lambda}{2 D}
$$

for a phase coherent real aperture, and

$$
\rho_{\text {along-track }}=\frac{R_{0} \lambda}{D} \text {. }
$$

for an incoherent real aperture. 
Apertures could, of course, be made indefinitely long even with changes in phase due to medium instability but this leads to a smearing of the image. The only way to recover from the phase instability is by having some form of autofocusing scheme which optimizes (sharpens) the target images (see Loggins et alia [1982], or to use one of several phase correcting techniques (see Sato et al. [1977], Ikeda et al. [1979], Ikeda et al. [1980] and Ikeda et al. [1985] ). Autofocusing techniques could, in principle, be used to recover from both medium instability and from platform motion. See Blackwell and Quegan [1990] for discussion an autofocusing for SAR purposes. Note that the medium stability issues discussed thus far for SAS have no significant counterpart in SAR.

Since the subject of medium stability and its influence on synthetic apertures depends heavily on the behavior of the medium, let's turn to the previous work in medium stability which was postponed from the first chapter.

\subsection{Previous Work in Medium Stability}

While there are a greater number of theory-based papers on synthetic aperture sonar (SAS) than papers combining both theory and experiments, the opposite is the case for acoustical environment stability, or acoustical medium stability (medium stability for short). A tabular summary and comparison of scveral relevant experiments is made in section 4.3.1, and then the experiments are more fully described in section 4.3.2. A summary and analysis of propagation path coherence, including a comparison of theory and experiment, may also be found in Chapter 4 of de Heering's Ph.D. thesis [1989].

\subsubsection{Summary and Comparison of Medium Stability Experiments}

Many experiments have been conducted concerning acoustic medium stability. The experiments especially related to synthetic aperture sonar (either active or passive) are shown in Table 4.1, and several other related medium stability experiments are referenced in the bibliography. 
Table 4.1 - Medium Stability Experiment Summary

\begin{tabular}{|c|c|c|c|}
\hline frequency & total path (km) & $\begin{array}{c}\sigma_{\mathrm{ms}} \\
+/ \text { - degrees }\end{array}$ & $\begin{array}{l}\text { coherence time } \\
\text { (seconds) }\end{array}$ \\
\hline Wequency & toral paut (KMiI) & & \\
\hline
\end{tabular}

Christoff et alia, 1974

near surface

$100 \mathrm{kHz}$

0.048

17.8

120

near bottom

"

"

2.3

"

Gough and Hayes, 1989

15- to $30-\mathrm{kHz} \quad 0.130$

10.

60

Stowe et alia, 1974

$10 \mathrm{kHz}$

2.5 a

3.5

60

Williams, 1976

$400 \mathrm{~Hz}$

$107-495$

49. $b$

148 to 450

Williams and Battestin, 1976

$400 \mathrm{~Hz}$

$270-1200$.

90.

120 to 480 typical

Yen and Carey, 1989

$175 \mathrm{~Hz}$

$195-250$

$<540$

Fitzgerald et alia, 1976

$10.04 \mathrm{~Hz}$

$65 \cdot 71$

15. ${ }^{b}$

1000

a. Nearly vertical path. All other experiments shown here had predominantly horizontal propagation.

b. K.D. Rolt estimate.

The results shown in Table 4.1 may be arranged to form a dimensionless quantity, in order to better compare these experiments which were conducted under different conditions, at different frequencies, and at differing ranges. The path length for each experiment may be reduced to the number of wavelengths by dividing the average total path (in meters) by the wavelength (also in meters). This result is then multiplied by the nominal center frequency (in Hertz) and by the coherence time $\mathrm{T}_{\text {cohere }}$ (in seconds). This gives a dimensionless number $\Phi$ to compare one experiment to another:

$$
\Phi=\frac{\text { total path }}{\lambda} \mathfrak{f}_{c} T_{\text {cohere }}
$$

To make the comparison still further equal, we would also like to compensate for the differing values of $\sigma_{\mathrm{rms}}$ in each experiment. One would expect that the measured coherence time would be smaller for 
r.duced values of $\sigma_{\mathrm{rms}}$, and so we assume a linear decrease in $T_{\text {cohere }}$ for reduced-scale estimates of $\sigma_{\mathrm{rms}}$. Choosing the normalized reference to $\sigma_{\mathrm{rms}}= \pm 10^{\circ}$ from the Gough and Hayes experiment, we find that:

$$
\begin{aligned}
& \chi=\Phi\left[\frac{10^{\circ}}{\sigma_{\mathrm{rms}}}\right] \\
& \tilde{\chi}=\Phi\left[\frac{10^{\circ}}{\sigma_{\mathrm{rms}}}\right]^{2}
\end{aligned}
$$

where $\chi$ and $\tilde{\chi}$ are the linear and quadratic estimates.

The calculated values for $\Phi, \chi$ and $\tilde{\chi}$ for the experiments in Table 4.1 are shown in Table 4.2. The results for the dimensionless quantity $\chi$, as adjusted for equal phase variance $\sigma_{\mathrm{rms}}$, provides a qualitative comparison between experiments. The near-bottom portion of the Christoff $e t$ alia experiment shows the best stability (the maximum value for $\chi$ ). This may he attributed to two features of their experiment:

- the measurement was taken on a rail-based platform, and so phase variation due to platform motion is effectively removed;

- the near-bottom measurement avoided the acoustical instability due to surface mixing. This is observed by comparing $\sigma_{\text {rms }}$ for the near-surface and near-bottom measurements; the phase variation was degraded by one order of magnitude at the surface as compared to the measurement taken close to the bottom. (However, the $\pm 17.8^{\circ}$ phase variation is still considered small per Table 4.1).

The experiment of Stowe et alia was performed with precision rivalling the rail-based experiment of Christoff $e t$ alia. This is not too surprising since the source was transmitted from a platform having a very high accuracy, on-board inertial navigation unit, and the receiver(s) were the bottom-moored hydrophones in the AUTEC test range (see description which follows). The test range is extensive and is often used to measure stbmarine self noise, and to evaluate submarine weapons performance. The relative agreement of $\chi$ and $\tilde{\chi}$ between the Christoff and Stowe experiments suggests an upper bound on phase stability (within measurement error, and without platform motion.)

The Gough and Hayes experiment also had the benefit of platform stability due to the wire guides. The remaining experiments in Table 4.2 were performed with various forms of towed sources or receivers, and had no form of platform stabilizing rails, or guides. Thus, these stability measurements are contaminated 
by source platform, tow ship, and receiver platform motion. The values of $\chi$ and $\tilde{\chi}$ broadly showed reduced stability as compared to the previous three experiments.

Table 4.2 - Medium Stability Experiments Compared

$\begin{array}{ccc}\Phi & \chi & \tilde{x} \\ \times 10^{9} & \times 10^{9} & \times 10^{9}\end{array}$

Christoff et alia, 1974

near surface

38.4

21.6

12.1

near bottom

38.4

167.0

725.0

Gough and Hayes, 1989

2.6

2.6

2.6

Stowe et alia, 1974

10.0

28.6

81.6

Williams, 1976

9.6

2.0

0.08

Williams and Battestin, 1976

23.5

2.6

0.29

Yen and Carey, 1989

2.5

Fitzgerald et alia, 1976

.005

.0005

0.00006

Remarks: 1. $\chi$ and $\tilde{\chi}$ normalized to Gough \& Hayes $\sigma_{\mathrm{rms}}=10^{\circ}$.

Several other medium stability experiments have been conducted, but without sufficient information to augment the data in Tables 4.1 and 4.2.

\subsubsection{Experiment Descriptions}

A seldom cited, but important, reference work on medium stability which concerns SAS is that by Stowe et alia [1974], based on a 1972 experiment conducted in the Atlantic Undersea Test and Evaluation 
Center (AUTEC) acoustic range. The experiment was based on a report by Chramiec and Walsh [1971] of the Raytheon Submarine Signal Division in which a medium stability experiment was proposed. The intent was to measure the phase stability of the acoustic medium, which would suggest the ultimate resolution capability of a SAS system, and to determine the measurement accuracy needed for platform motion compensation. The experiment used an acoustic source (or a projector) near the ocean surface (70 $\mathrm{m}$ depth) which was the received by an array of bottom-mounted hydrophones, and the one-way travel time was measured. The path distance was about 2500 meters, and the angle to the horizontal was $45^{\circ}$ or more so the acoustic path was predominantly vertical. The travel time was corrected for projector motion because the motion of the projector was measured via a state-of-the-art inertial measurement unit (IMU). The remaining flucivations in travel time then represent "residual measurement errors and fluctuations of the acoustic path in the ocean itself." The result was that the rms fluctuation of the acoustic path was about $\lambda / 100$ at $10 \mathrm{kHz}$ over a one minute period, which is about a $3.5^{\circ}$ phase angle.

Spindel, Porter, and Jaffee [1974] showed the results for a long-range phase fluctuation experiment using a bottom moored $406 \mathrm{~Hz}$ source ( 4800 meter depth), ship-suspended hydrophones ( $1500 \mathrm{~m}$ depth) and drifting sonobuoys (305 $\mathrm{m}$ depth) at a nominal range of 210 kilometers. The maximum phase fluctuation was approximately 15 cycles over 3 hours for the $1500 \mathrm{~m}$ depth hydrophones, and about 7.5 cycles for the $305 \mathrm{~m}$ depth sonobuoys for the one-way source to receiver path. The authors suggested that their result "..opens the door to synthetic aperture generation through moving sources or receivers, thus greatly simplifying space-time coherence investigations."

Shaffer, Fitzgerald, and Guthrie [1974] compared the theory of the dependence of the phase of low frequency cw sound on depth and range, and compared it with data from two experiments. A lower bound on coherence in time was determined from a lower bound of coherence in range. For a source-receiver distance of ahout $1000 \mathrm{~km}$, a $9 \mathrm{~km}$ range interval was observed where the phase was a linear function of range. Since the ship speed was about $13 \mathrm{~km} / \mathrm{hr}$, this implies that the signal was temporally coherent for at least 40 minutes.

Fitzgerald, Guthrie and Shaffer [1976] reported experimental evidence from a 1960 experiment showing the coherence of $10 \mathrm{~Hz}$ signals measured off the Hatteras abyssal plain. The projector was towed at a $25 \mathrm{~m}$ depth and the receiver was suspended at $460 \mathrm{~m}$ depth from a drifting radio buoy. The water depth was over $5000 \mathrm{~m}$ deep. They concluded from the results of measuring the phase-versus-time data that the signal was coherent to within $45^{\circ}$ during an interval of about 2100 seconds (35 minutes), and was coherent to within $30^{\circ}$ during a number of 1000 second (about 17 minute) intervals. Since the towing ship's speed was $1.6 \mathrm{~m} / \mathrm{s}$, the possible synthetic aperture lengths are 3360 meters and 1600 meters respectively for these two coherence times. 
Williams and Battestin [1976] presented long-term time coherence measurements of broad-band acoustic signals in a deep-ocean environment between Bermuda and Eleuthera islands which were conducted during the summers of 1969 and 1970. The distances were 270-, 466-, 922-, and 1182-km (145-, 250-, 495-, and 635-nautical miles). The source was suspended from a ship and the receiver was a vertical hydrophone array moored to the ocean bottom. The hydrophone array was used to discriminate individual ray paths. Typical coherence intervals of 2 to 8 minutes were found for ray paths where the phase of a replica-correlation of the received signal varied by less than $+/-\pi / 2$ at $400 \mathrm{~Hz}$. Furthermore, longer coherent intervals were common, typically from 8 to 12 minutes and occasionally up to 20 minutes long. The acoustic paths over which these results were found were assumed to be refracted surface-reflected (RSR) and suggest that the ocean is sufficiently well-behaved to support the use of "extended coherence apertures". Preliminary results from this experiment were previously reported by one of the authors [Williams, 1970].

Williams [1976] also showed coherence data from a passive synthetic aperture experiment using 400 $\mathrm{Hz} \mathrm{cw}$ and 350- to 450-Hz FM towed sources and moored, midwater buoyed hydrophones. Only the $400 \mathrm{~Hz}$ cw source showed good coherence, and the lack of coherence in the.FM source data was attributed to motion of the source due to influence from ship yaw, and to vortex shedding from the source. The coherence lengths for the $\mathrm{cw}$ data ranged from $350 \mathrm{~m}$ to $1158 \mathrm{~m}$, with coherence times of 148 - to 450 -seconds.

Spiesberger, Spindel and Metzger [1980] also performed a long-range experiment to evaluate the stability and identification of ocean acoustic mulupaths using a phase-coded signal. The transmission was over a deep ocean channel at a range of $900 \mathrm{~km}$, between a source moored at a $2000 \mathrm{~m}$ depth and a receiver moored at a $3000 \mathrm{~m}$ depth. Approximately 16 multipaths were resolved and could be substantially identified from ray theory.

Christoff, Loggins, and Pipkin [1982] measured the phase stability at the site used for their rail-based synthetic aperture sonar experiment. The measurements were conducted over a two-year period and were made at a number of locations between the surface and the bottom. The measurement was performed bistatically with a $48 \mathrm{~m}$ separation between projector and hydrophone in about $12 \mathrm{~m}$ of water. The transmit frequency was $100 \mathrm{kHz}$. Their results showed that the standard deviation of the phase was small for nearbottom measurements, but was much larger as the surface was approached. For a 20 minute interval and measured 3 meters off the bottom, the phase standard deviation was found to be as small as 0.04 radians $\left(2.3^{\circ}\right)$. In a separate measurement $3 \mathrm{~m}$ from the surface and over a 2 minute period, the phase standard deviation was .31 radians $\left(17.8^{\circ}\right)$. 
Gough and Hayes [1989] measured the acoustic phase stability from a pier in a Scottish Loch over the period of several days, as a companion to a SAS experiment. They observed that over elapsed time periods of one minute, the standard deviation of the phase fluctuations about the mean phase was $10^{\circ}$. They also found that the standard deviation of the acoustic phase was independent of frequency, over a 15- to $30 \mathrm{kHz}$ band, which implied that the standard deviation measured is limited by the measurement accuracy. So the actual standard deviation of the acoustic phase stability is better than $10^{\circ}$ for all frequencies. Their measurements were made over an out-and-back path of about $\mathbf{1 3 0}$ meters. This is a remarkable result, especially in view of the location of the experiment. The authors stated that Loch Linnhe "is a sea loch with considerable tidal flows (up to $6 \mathrm{kn}$ at the pier end) and has a significant amount of fresh water inflow after rainfall." Gough and Hayes remarked that their results are on the same order of magnitude as those determined by Christoff $e t$ alia, and are beuter than had been previously surmised.

Yen and Carey [1989] also found medium stability from a SAS experiment, though theirs was a passive SAS experiment, and was over considerably longer ranges (up to $250 \mathrm{~km}$ ) than for Gough and Hayes. The synthesized array was able to form good beams on the moored source for lengths between 192 and $962-\mathrm{m}$, in deep water (3200 meters). The coherent processing time for these array lengths was less than 9 minutes.

Urban and Stergiopoulos [1990] reported on the spatial and temporal coherence of an ocean region where a passive synthetic aperture experiment was conducted.

For other relevant medium stability results see Parkins and Fox [1971], McKean [1974], Shaffer $e t$ alia [1974], Spiesberger et alia [1980], Spindel [1980], and Worcester [1981].

\subsection{Platform Motion}

As we have just shown, the medium plays an important role in determining the maximum length of a synthetic aperture, and thereby what its actual resolution limits are, depending on the range to the target. The changes in the properties of the medium, over space and over time (spatial and temporal) result in a change in the sound speed profile which has the consequence of changing the number of wavelengths from a source to a target, and this is a change in phase. Another mechanism for changing the path length between a source and a target is the motion of the sonar platform. 
When the theoretical parameters such as resolution (in both along-track and cross-track) and sidelobe levels were developed for the cases of real, focused, unfocused, and spotlight apertures, a basic assumption was made that the sonar platform traveled on a straight-line path at constant velocity $V_{0}$. This can be made to happen, and has been done in a number of synthetic aperture sonar experiments by guiding the platform on a wire or rail, and propelling it by external means. If however, we had a sonar platform which was selfpropelled, and unattached to any type of guideway, the platform would not move exactly on a straightline path, or at constant velocity, even if the water environment were calm. To make matters worse, if the water environment has any current present, which is very reasonable, this will further impair the ability of the platform to travel along a straight line. These influences of the medium upon the platform motion are not restricted to undersea travel, but also exist for the aircraft (which also travel in a fluid: the atmosphere) and for spacecraft.

It is usually necessary to have on-board sensors to determine what the motion of the platform is, so that the echo data used in the apenture synthesis may be corrected for the platform motion. This converts data laden with platform-motion errors to data free from motion errors. The demands on the accuracy of the measuring sensors (accelerometers, for example) depend on the stability of the platform and on the wavelengths of sound (or radar) being used to form an image. The stability of the platform is determined by the combination of the inherent platform stability and by the controllers on-board. This may differ from platform to platform and also depend on how sophisticated (i.e. expensive) the controllers are. We will ignore these things, and concentrate on the effect of wavelength.

This analysis which follows takes the approach and notation of Cutrona [1975], but also shows the intermediate steps. Cutrona starts with the basic premise that the variance of platform displacement in the cross-track (broadside) direction is proportional to the variance in cross-track acceleration and to the square of time t:

$$
\sigma_{\mathrm{r}}=\frac{\sigma_{\mathrm{a}}}{2} \mathrm{t}^{2}
$$

The derivation of (4.9) is found in Appendix E. Equation 4.9 takes the form of

$$
\mathrm{s}=\frac{1}{2} \mathrm{at}^{2}
$$

where $\mathbf{s}$ is displacement and $\mathbf{a}$ is acceleration. 
From the discussion in Chapter 2, the length over which an aperture may be coherently synthesized is

$$
\mathbf{L}=\mathbf{R}_{\mathbf{0}} \frac{\lambda}{\mathrm{D}}
$$

and since $L=V_{0} T_{\text {sas, }}$, then

$$
T_{\text {sas }}=\frac{R_{0} \lambda}{V_{0} D}
$$

$T_{\text {sas }}$ is the time required for the platform to travel the distance $L$. We now let the position variance $\sigma_{\mathrm{r}}$ of (4.9) be given by

$$
\sigma_{\mathrm{r}}=2 \varepsilon \lambda \text {. }
$$

The variable $\varepsilon$ in (4.12) is a small quantity which is usually set equal to 1/8 in the SAR literature. This makes $\sigma_{\mathrm{r}}$ equal to $\lambda / 4$, which is the allowable two-way error in slant range for an unfocused SAR (or SAS), and which we also use here for the limit on platform motion measurement error. Since we let $\varepsilon=1 / 8$, for the one-way error, we then only use half the elapsed transit time $\mathrm{T}_{\text {sas. }}$ In review,

$$
\begin{aligned}
& \sigma_{\mathrm{r}}=2 \frac{1}{8} \lambda=\frac{\lambda}{4}, \\
& t=\frac{T_{\text {sas }}}{2}=\frac{R_{0} \lambda}{2 V_{0} D} .
\end{aligned}
$$

We now substitute (4.13) and (4.14) into (4.9) and solve for $\sigma_{\mathrm{a}}$ :

$$
\sigma_{\mathrm{a}}=\frac{\lambda}{2}\left(\frac{4 \mathrm{~V}_{\mathrm{o}}}{\mathrm{R}_{\mathrm{o}} \lambda} \frac{\mathrm{D}}{2}\right)^{2}
$$

We now substitute $\delta_{\mathrm{az}}=\rho_{\mathrm{along}}$-track $=\mathrm{D} / 2$, and find the result that

$$
\sigma_{\mathrm{a}} \leq \frac{8 \delta_{\mathrm{az}}{ }^{2} \mathrm{~V}_{\mathrm{o}}{ }^{2}}{\mathrm{R}_{\mathrm{o}}{ }^{2} \lambda}
$$


This is essentially the result given by Cutrona (he used an equal sign however) showing the necessary sensitivity of the accelerometer (in terms of the accelerometer variance $\sigma_{\mathfrak{a}}$ ). Note that an inequality symbol is used instead of the equaiity, because the accelerometer variance must be less than or equal to the term shown above for useful measurement of the platform motion. 
- experiment of Gough \& Hayes

(J. Acoust. Soc. Am. 8 6(6) 1989)

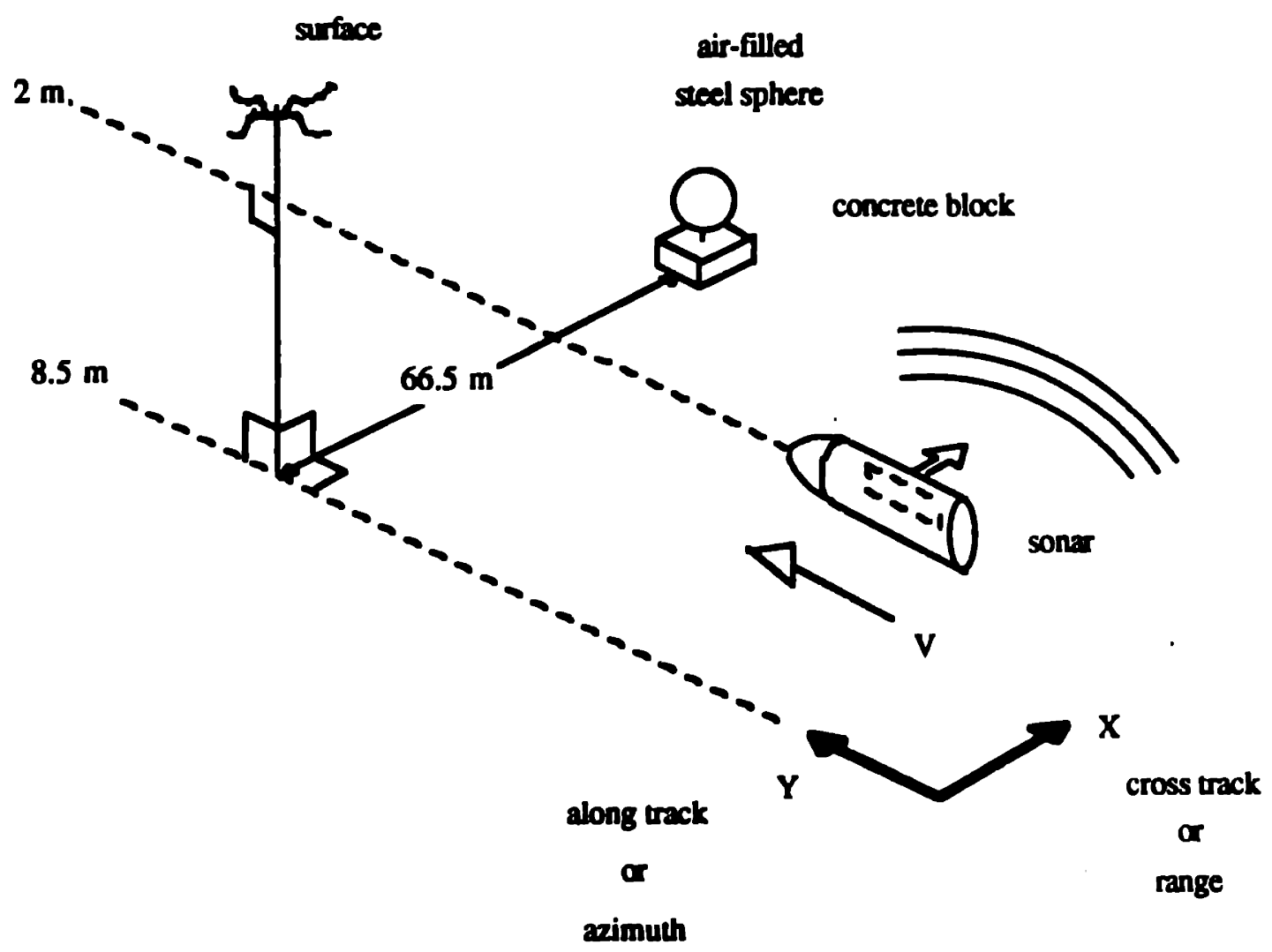

Figure 5.1 - Geometry for Gough and Hayes Experiment 


\section{Chapter 5}

\section{Model Validation and Examples}

This chapter presents examples of simulated synthetic aperture sonar images using the computer models create.out and synth.out. The effects of the ocean medium, the effects of the platform motion, and the effects of signal processing are demonstrated and discussed. Extensive validation of model examples via comparison with available experimental results is an integral part of this chapter.

\subsection{Model Verification}

The acid test for any ocean acoustic computer model is a comparison between its results and the results from a real experiment. The unclassified publication of the experimental SAS results of Gough and Hayes [1989] in the Journal of the Acoustical Society of America allows such a comparison to be made here, thus verifying the computer simulation at least for the conditions of frequency, range, and medium coherence encountered in the experiment. The geometry for their experiment is shown in Figure 5.1. The computer model geometry emulates the experiment wherever possible; the main exception is that in the real experiment, the target was a 1.2 meter diameter air-filled steel sphere which was suspended from a large concrete block at about 66.5 meters (ground plane) from the sonar track. The computer model uses an idealized point scatterer which is independent of incident angle and frequency. Figure 5.2 shows the computer model geometry, and shows the idealized, corner reflector point target.

\subsubsection{Focused (Gough and Hayes Comparison)}

Two focused examples are shown here for comparison with the experimental images of Gough and Hayes [1989]. Focused operation was covered in section 2.2.1. The operating parameters for the two cases from Gough and Hayes are shown in Table 5.1, and were used to simulate their experiment. Note that the Gough and Hayes experiment was a showcase for two features: the first was the demonstration of continuous transmission frequency modulation (CTFM) with synthetic aperture sonar. The second feature was the demonstration of smearing the alias target im: jes (called artifacts by Gough and Hayes) due to the effects of broadband operation for an undersampled aperture. Note that the apertures synthesired in the Gough and Hayes experiment were always spatially undersampled. The CTFM feature is not modulud in the simulations shown in this thesis. 


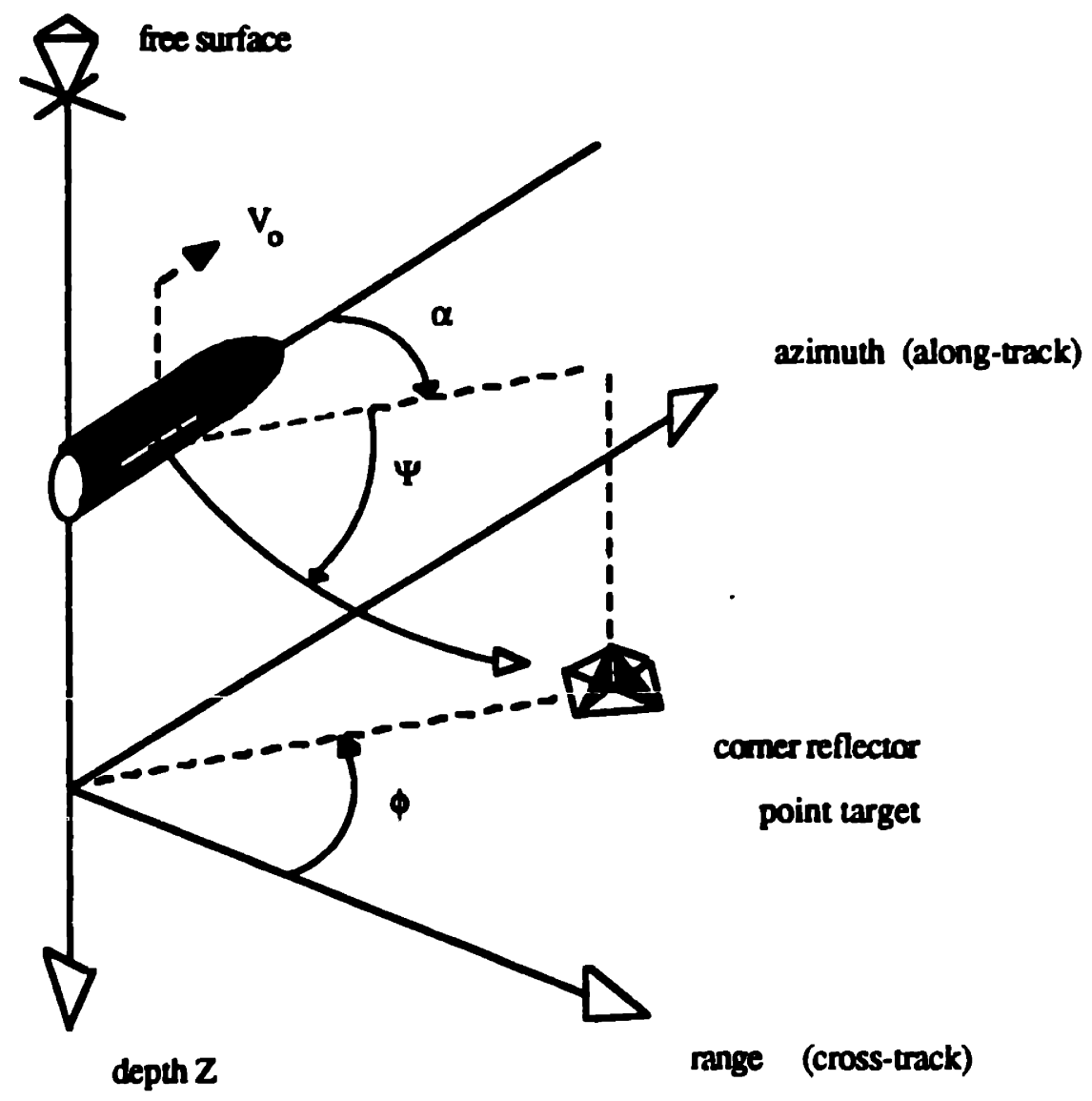

Figure 5.2 - SAS Geometry for Computer Model 
Table 5.1 - Operating Parameters for Gough and Hayes SAS

Used in the Computer Model

\begin{tabular}{ll}
\hline $\begin{array}{l}\text { sonar dimensions: } \\
\text { transmit chirp? }\end{array}$ & transceiver ${ }^{1}, .30$ meters horizontal, .050 meters vertical. \\
namowband & $\mathrm{f}_{\mathrm{c}}=28 \mathrm{kHz}, \mathrm{BW}=750 \mathrm{~Hz}$ \\
broadband & $\mathrm{f}_{\mathrm{c}}=22.5 \mathrm{kHz}, \mathrm{BW}=15 \mathrm{kHz}$ \\
source level & $190 \mathrm{~dB} \mathrm{re} 1 \mu \mathrm{Pa}$ at $1 \mathrm{~m}$. \\
PRF & $1.25 \mathrm{~Hz}$ \\
pulse duration & $40 \mathrm{milliseconds}$ \\
platform speed & $1 \mathrm{kn}(.517 \mathrm{~m} / \mathrm{s})$
\end{tabular}

notes:

1. Gough and Hayes used separate projector and receiver for CTFM; I used a single transceiver element in the computer model with no CTFM.

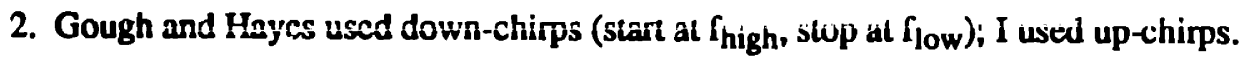

The first example, in Figure 5.3, shows the narrowband ( $750 \mathrm{~Hz}$ bandwidth, $28 \mathrm{kHz}$ center frequency) target image (the center peak) at an approximate slant range $x=66.8$ and along-track $y=65.0$, along with several alias targets. One of the aliases has a slightly larger magnitude than the actual target; this is most likely due to the location and spacing of the image points (the pixeis), where the image of an alias happened to give a slightly higher numerical value that for the target. Figure 5.3 may be compared with the intensity distribution result of Gough and Hayes (inset) as reprinted from the J. Acoust. Soc. Am. article (p. 2331). Gough and Hayes also found that one of the alias targets had a larger value than the target itself.

The resolution in both range and azimuth for the computer imagery agrees with that predicted by the theory developed in section 2.2 and summarized in Table 2.1. Note that in both the real experiment and in the simulation here, the images were generated from a single look $(\mathrm{N}=1)$ from the entire aperture length. Figure 5.4 shows the same image data as Figure 5.3, but now on a $\log$ scale $(\mathrm{dB})$ with the $0 \mathrm{~dB}$ reference as the largest image (in this case, an alias target). The target in this example is about $1.5 \mathrm{~dB}$ below the value for the alias target. Along-track slices (or azimuthal slices) of the image in $\mathrm{dB}$ will be presented later in this chapter, and will show the effects of using a sub-aperture for imaging, particularly for its influence on the alias targets. 


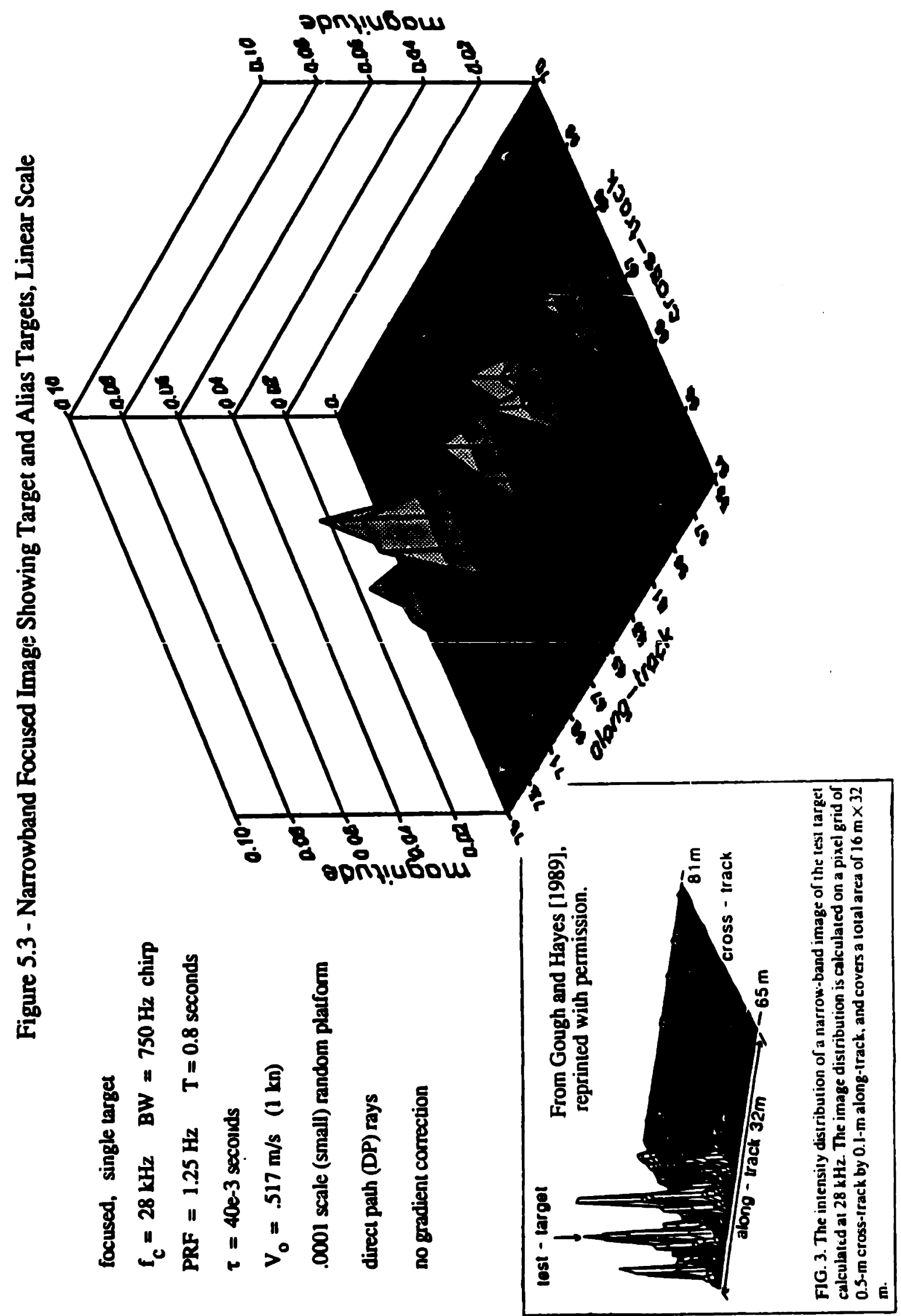




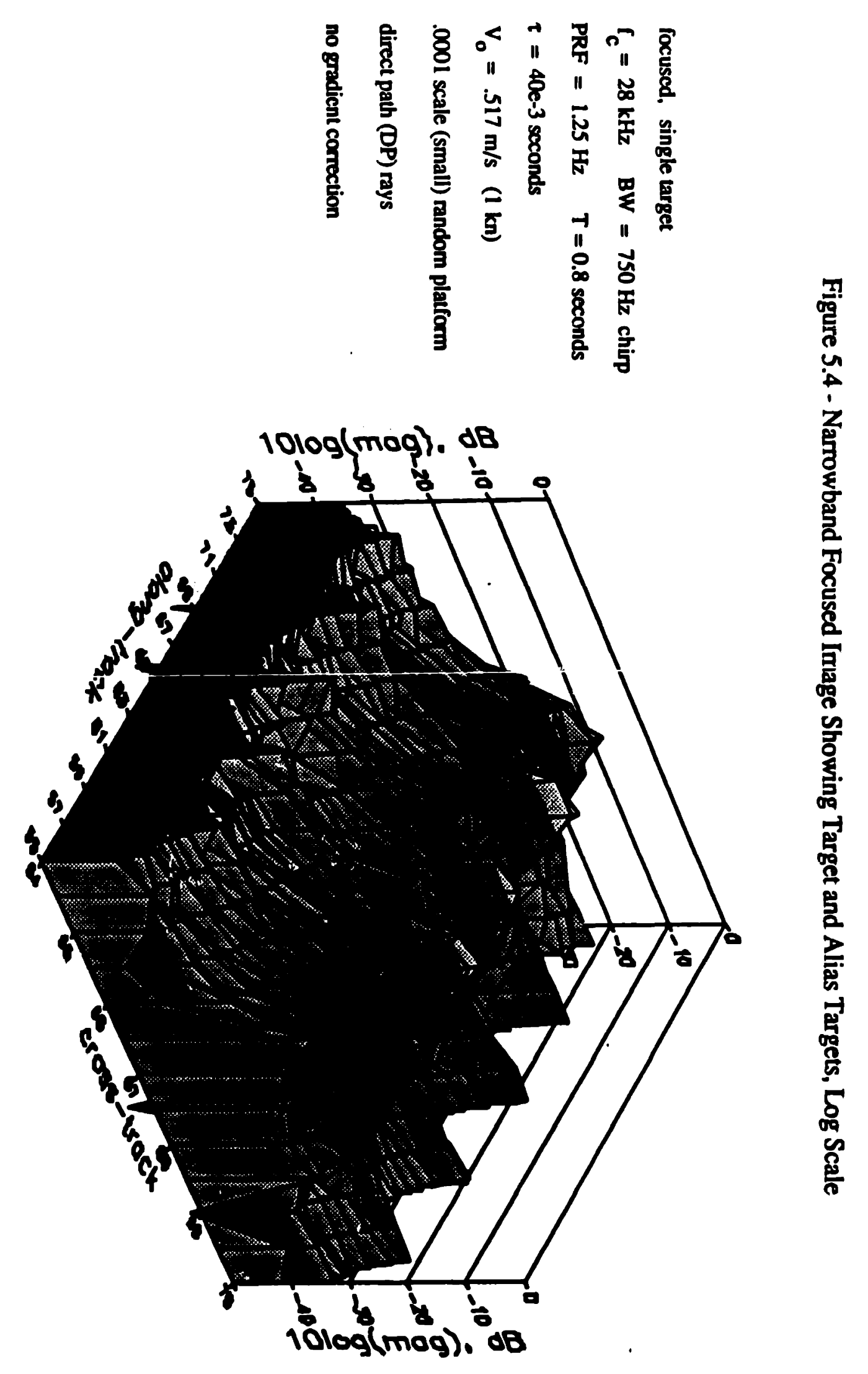


The second example, in Figure 5.5, shows the same scene as imaged from a single-look, broadband signal ( $15 \mathrm{kHz}$ bandwidth, $22.5 \mathrm{kHz}$ center frequency). Figure 5.5 is intentionally plotted on the same scale as the Hayes [1989] result (inset; see also Gough and Hayes [1989]) for the sake of a direct visual comparison.

Figure 5.6 shows the data of Fig. 5.5 plotted on expanded range and azimuth scales for clarity. Figure 5.7 shows the same data again, but now plotted on a dB scale with $0 \mathrm{~dB}$ as the target peak (azimuth from 64 to 66 meters, range from 65 to 68 meters). The superb range compression of the broadband signal is made obvious in Figure 5.7 by observing the sidelobes in range (more than $30 \mathrm{~dB}$ below the target). The sidelobe level in azimuth (along-track) is not smooth because there are not enough data (pixel) points to show smooth lobe structures; the level of the along-track sidelobes appears as a ridge at about - 12 to -15 dB, due to the influence of the nearby (smeared) alias lobes (which do not appear in the view of this Figure). However, these azimuthal aliases do appear however in Figure 5.8 (again, single look or $N=1$ ) in an along-track (or azimuthal) image slice taken through the target range (about 66.8 meters from the sonar) in both magnitude and $\log (\mathrm{dB})$ scales. Note that only one-half of the azimuthal image is shown; the target appears at 65.0 meters and the aliases (or alias 'ridge') appears from about 50 to 62.5 meters. An identical alias ridge would appear from 67.5 to 80 meters because they occur in symmetrical pairs about the target. Portions of the symmetrical ridge are shown in the Gough and Hayes experiment image (inset to Fig. 5.5).

Figure 5.8 shows that the smeared alias images have a $-6.5 \mathrm{~dB}$ maximum and generally reduced thereafter. Smeared alias images are also shown in the Hayes [1989] inset in Fig. 5.5; they appear as symmetric clusters of small peaks on either side of the target image peak. The inset in Fig. 5.5 may be directly compared to the magnitude scale along-track image slice in Figure 5.8 in this thesis. Note the pattern of a smooth flat region immediately adjacent to the target peak, followed by the elevated ridge of alias images.

The broadband imaging resolution in both the simulations shown here and in the Gough and Hayes experiment was about $5 \mathrm{~cm}$ in range and $17 \mathrm{~cm}$ in azimuth. The theoretical range and azimuth resolutions are 5 and $15 \mathrm{~cm}$ respectively. The first sidelobe level in range was $-35 \mathrm{~dB}$ (relative to $0 \mathrm{~dB}$ mainlobe) and the along-track first sidelobe level was $\mathbf{- 8 . 2} \mathrm{dB}$ (which is close to the $\mathbf{- 9 . 7} \mathrm{dB}$ estimated for the narrowband case in Table 2.3). 

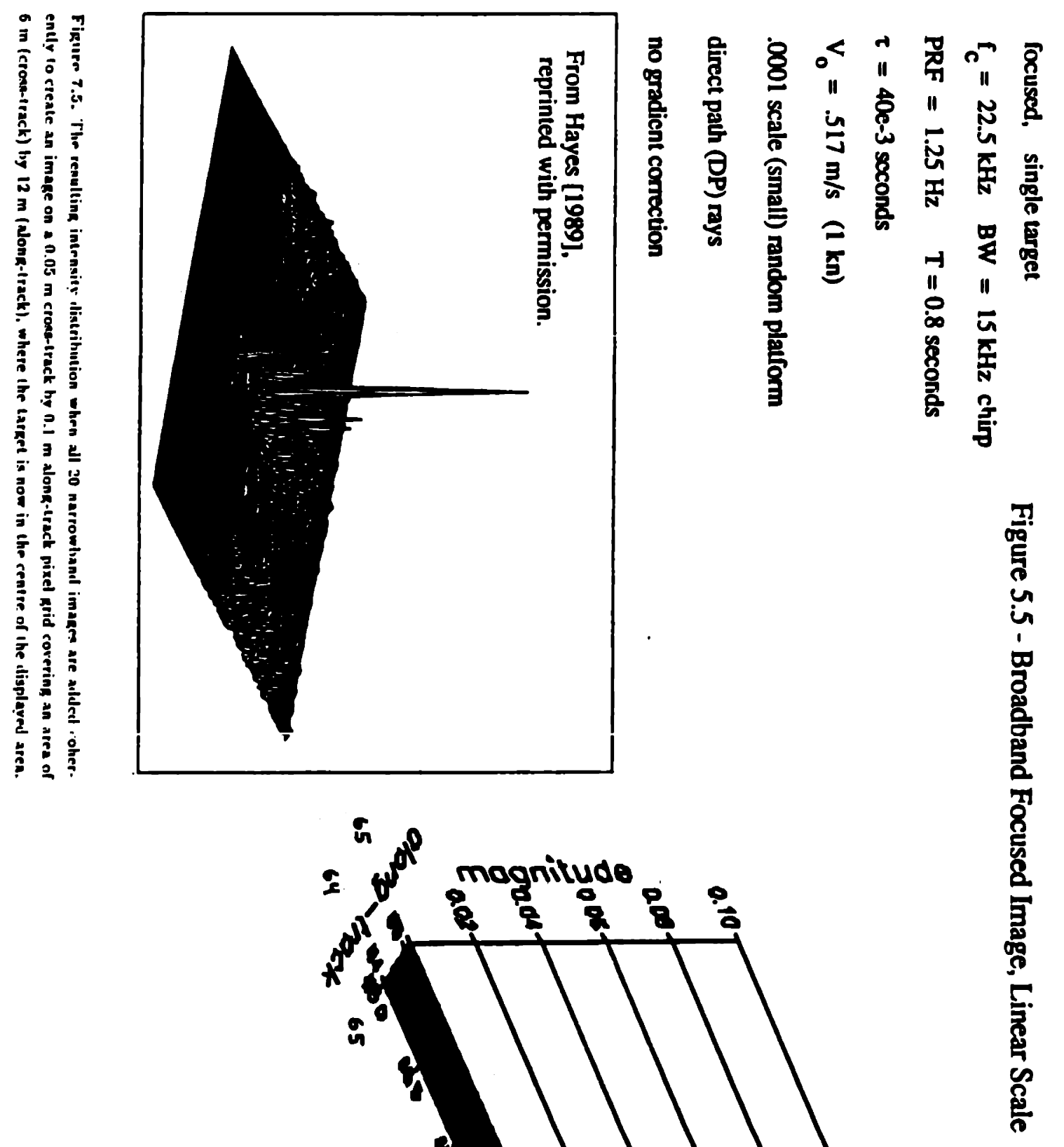


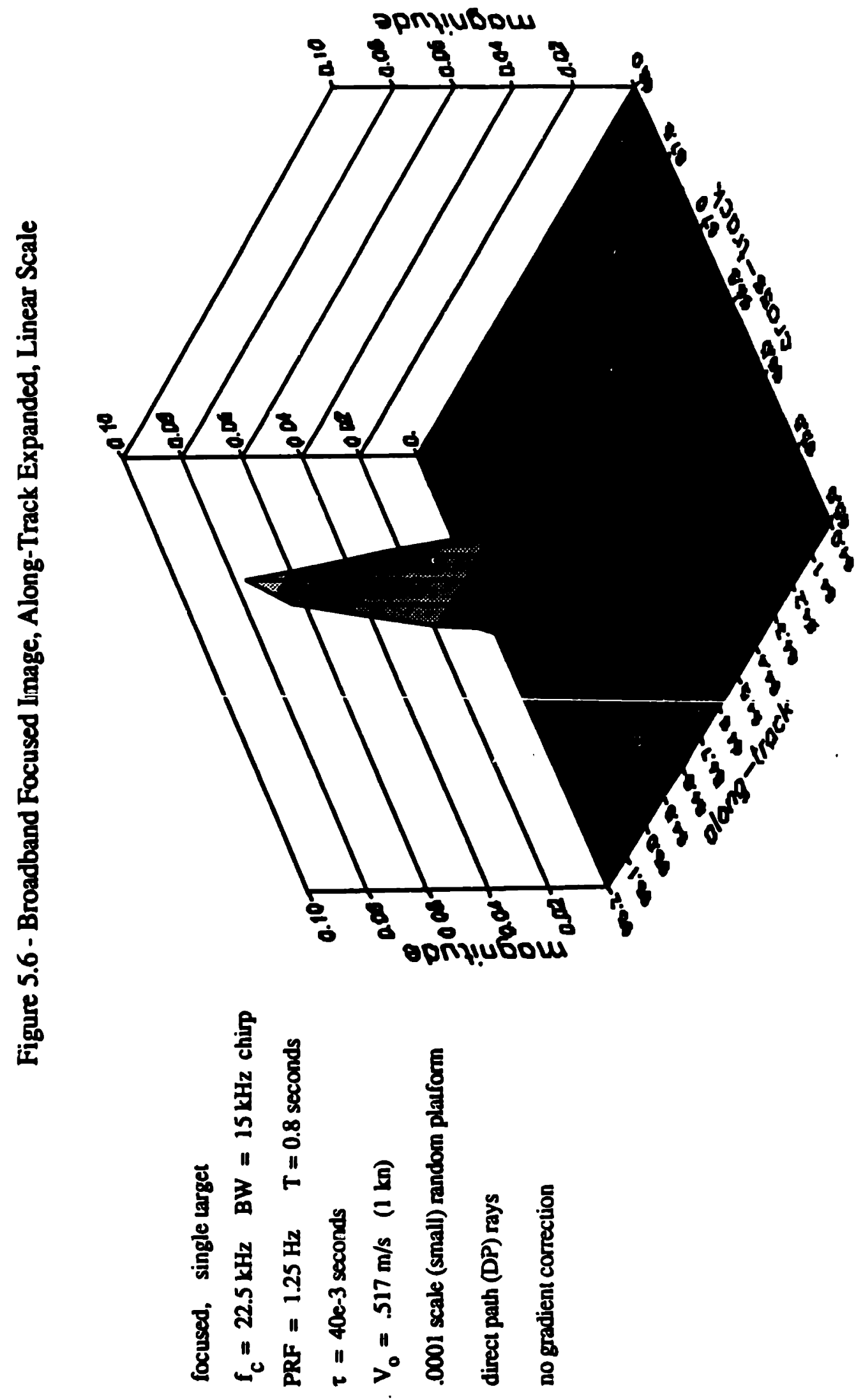




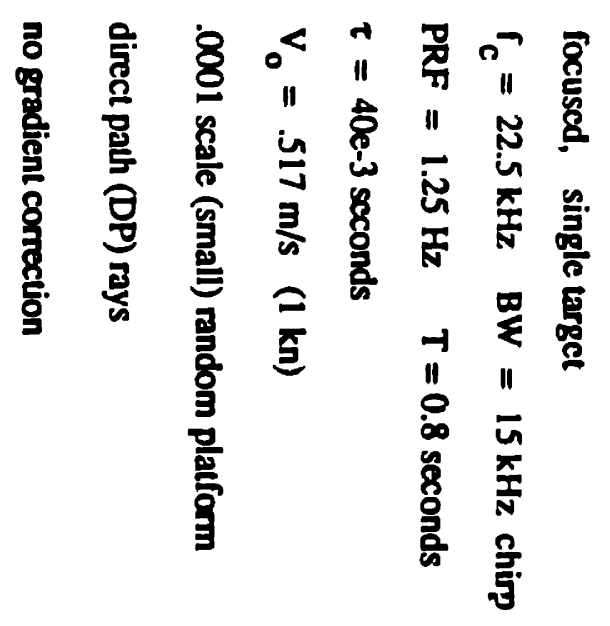

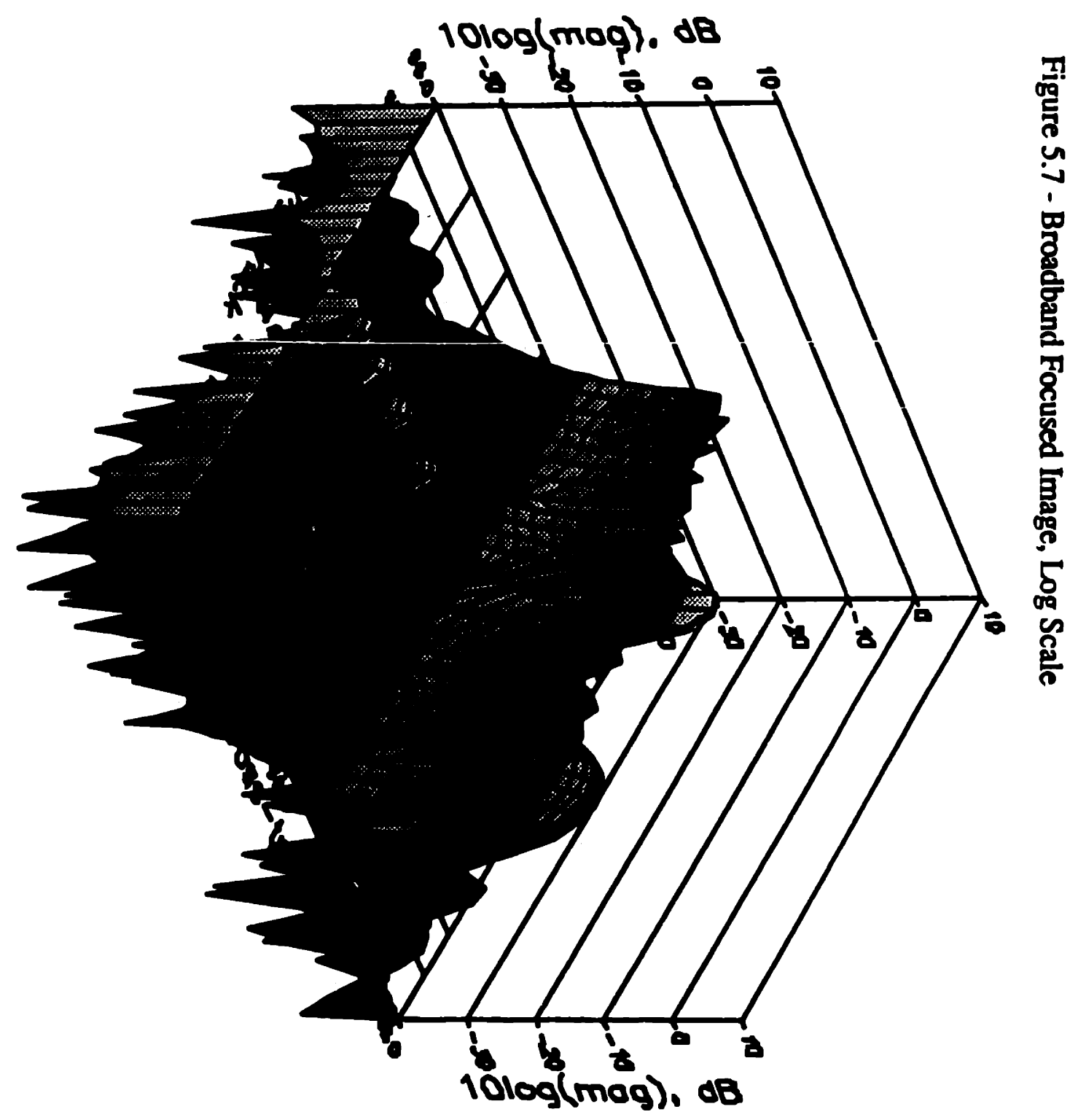




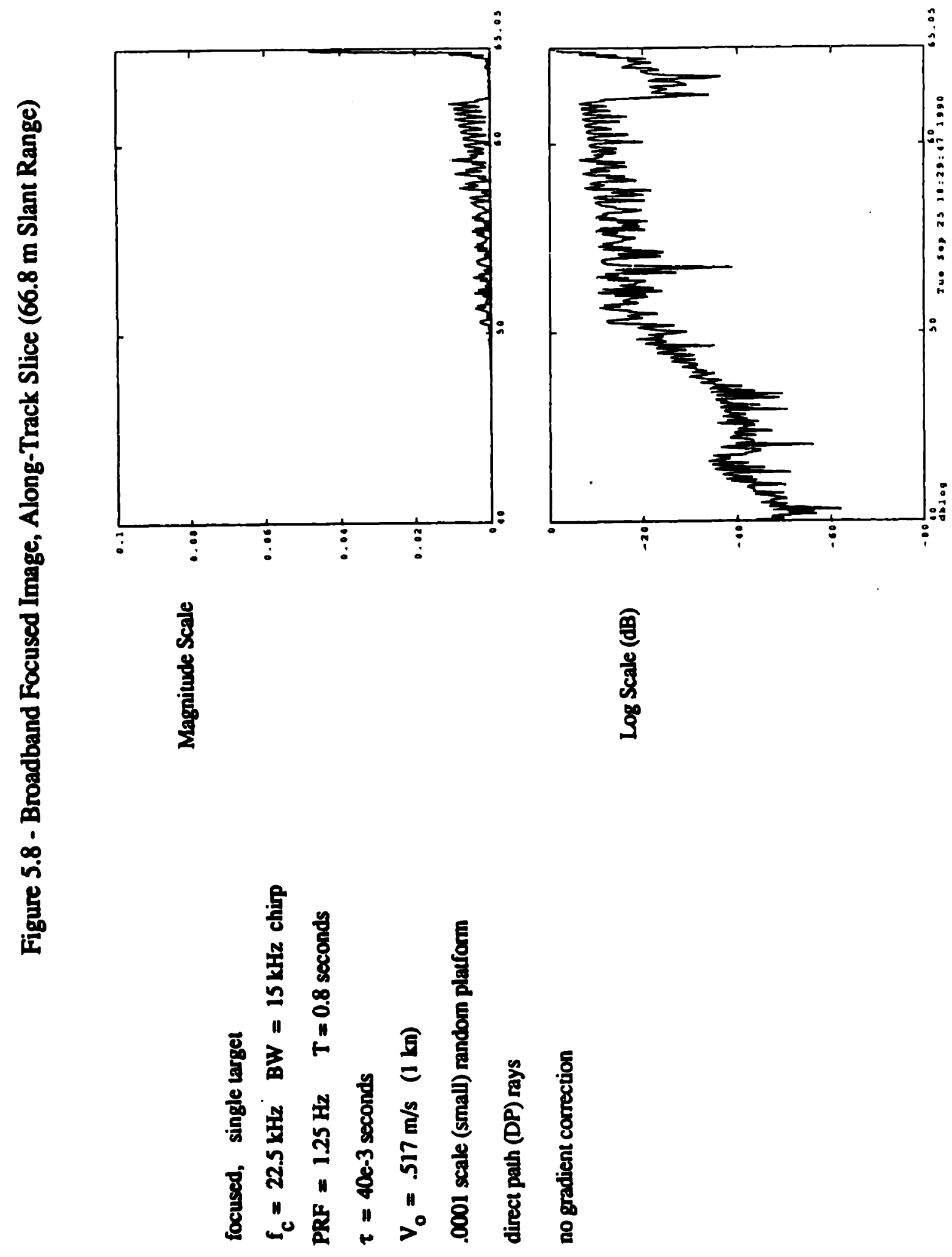




\subsubsection{Unfocused}

The previous section verified the computer model under focused operation (i.e. focus at all points in the slant-range/azimuth image space) by comparison with real data. This section shows images from the same examples of section 5.1.1, except that unfocused processing is used (as described in section 2.2.2; note that unfocused images were not created from the Gough and Hayes [1989] experiment data.)

Figures 5.9 and 5.10 respectively, show the magnitude and log-scale images of the target for the $f_{c}=28 \mathrm{kHz}, \mathrm{BW}=750 \mathrm{~Hz}$ narrowband case using unfocused processing. The along-track resolution is 1.2 $\mathrm{m}$ compared with $.93 \mathrm{~m}$ theoretical, while the slant-range resolution is about 1.0 meters for both the model and theory.

Figures 5.11 and 5.12 likewise show the respective magnitude and log-scale images of the target in the wideband case, where $f_{c}=22.5 \mathrm{kHz}$ and $\mathrm{BW}=15 \mathrm{kHz}$. The along-track resolution was about $1.5 \mathrm{~m}$, which may be compared with the along-track resolution at the extremes of the bandwidth: $.91 \mathrm{~m}$ for $30 \mathrm{kHz}$ and $1.28 \mathrm{~m}$ for $15 \mathrm{kHz}$. The deviation between the model and the theory is reasonable, since the theory expects a single wavelength transmitted signal, and because the maximum one-way $\lambda / 4$ measurement criterion (see Figure 2.11) for the unfocused array also depends on a single wavelength. The processor used to form these images uses an average wavelength, so there is an approximation involved which slightly reduces the resolution from the theoretical value. The range resolutions for both cases were not measurably different than for the focused cases shown in 5.1.1.

To recall from section 2.2.2, the unfocused synthetic aperture forms a target image using less of the azimuthal data (and less computation time), at the expense of less azimuthal resolution. The azimuthal resolution capability is proportional to the square root of the the range-wavelength product, contrasting the focused azimuthal resolution which is constant with range and frequency independent, and contrasting the azimuthal resolution for a real aperture which is proportional to the range-wavelength product. The unfocused synthetic aperture compromises azimuth resolution for imaging speed, and may be useful for initial large-scale fast imagery to identify key regions. These smaller key regions may then be reprocessed using focused techniques to recover the full azimuth resolution. 

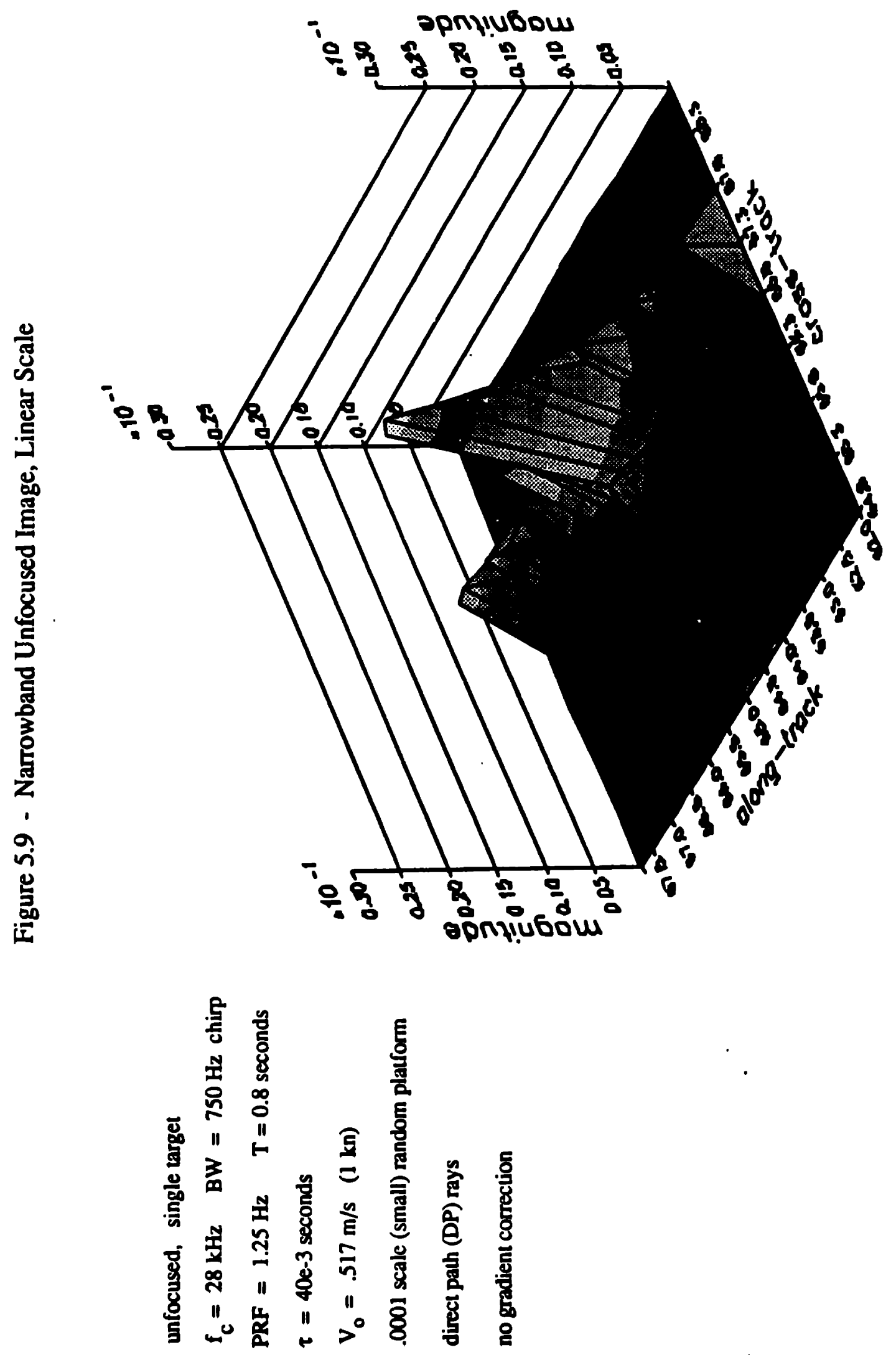

152 


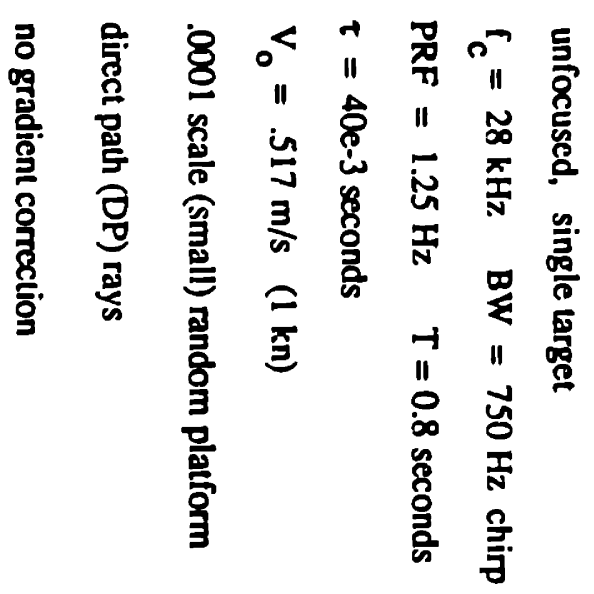

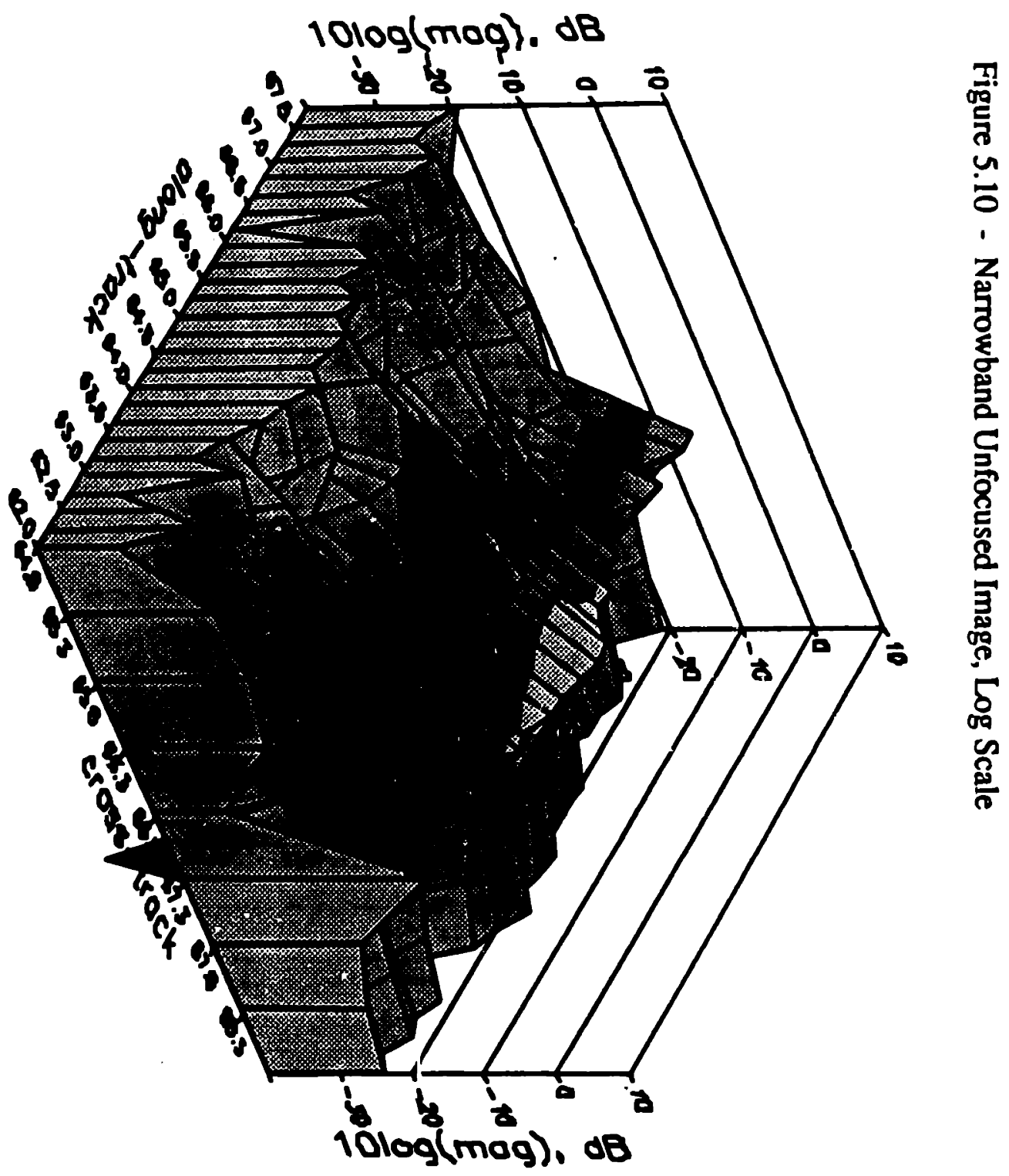




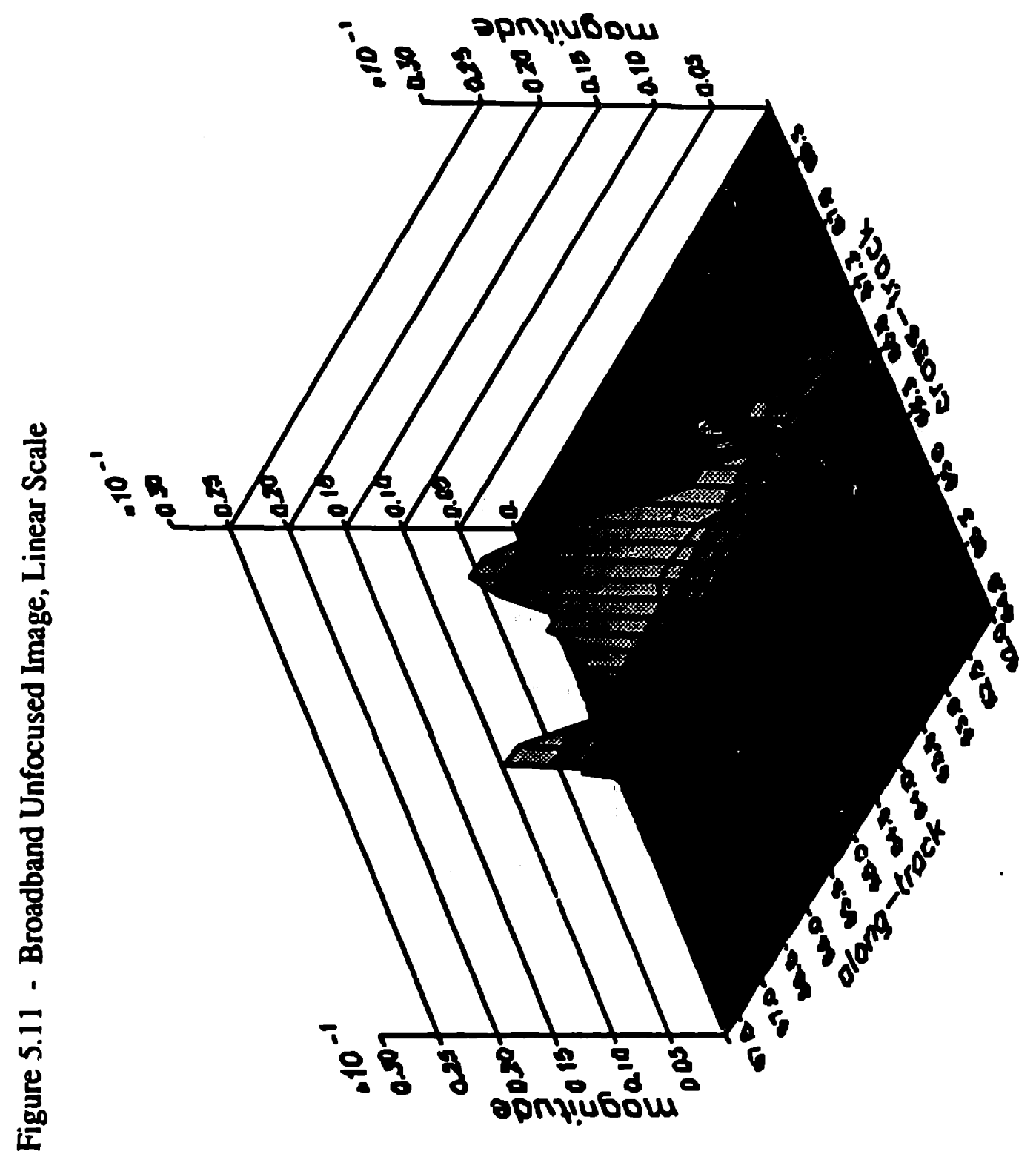

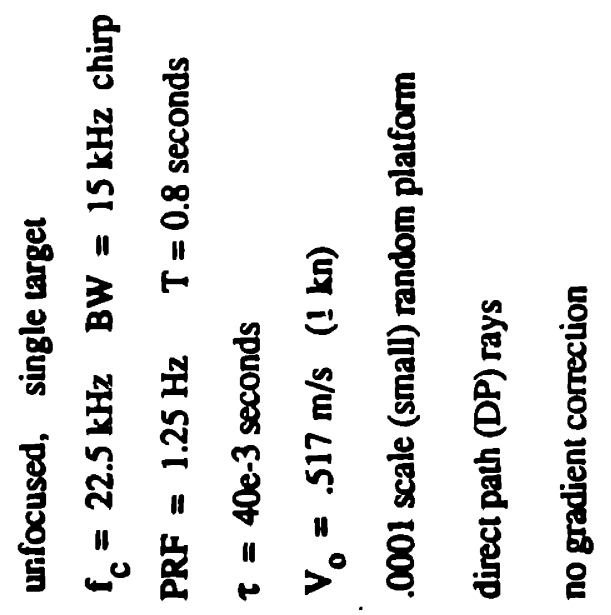




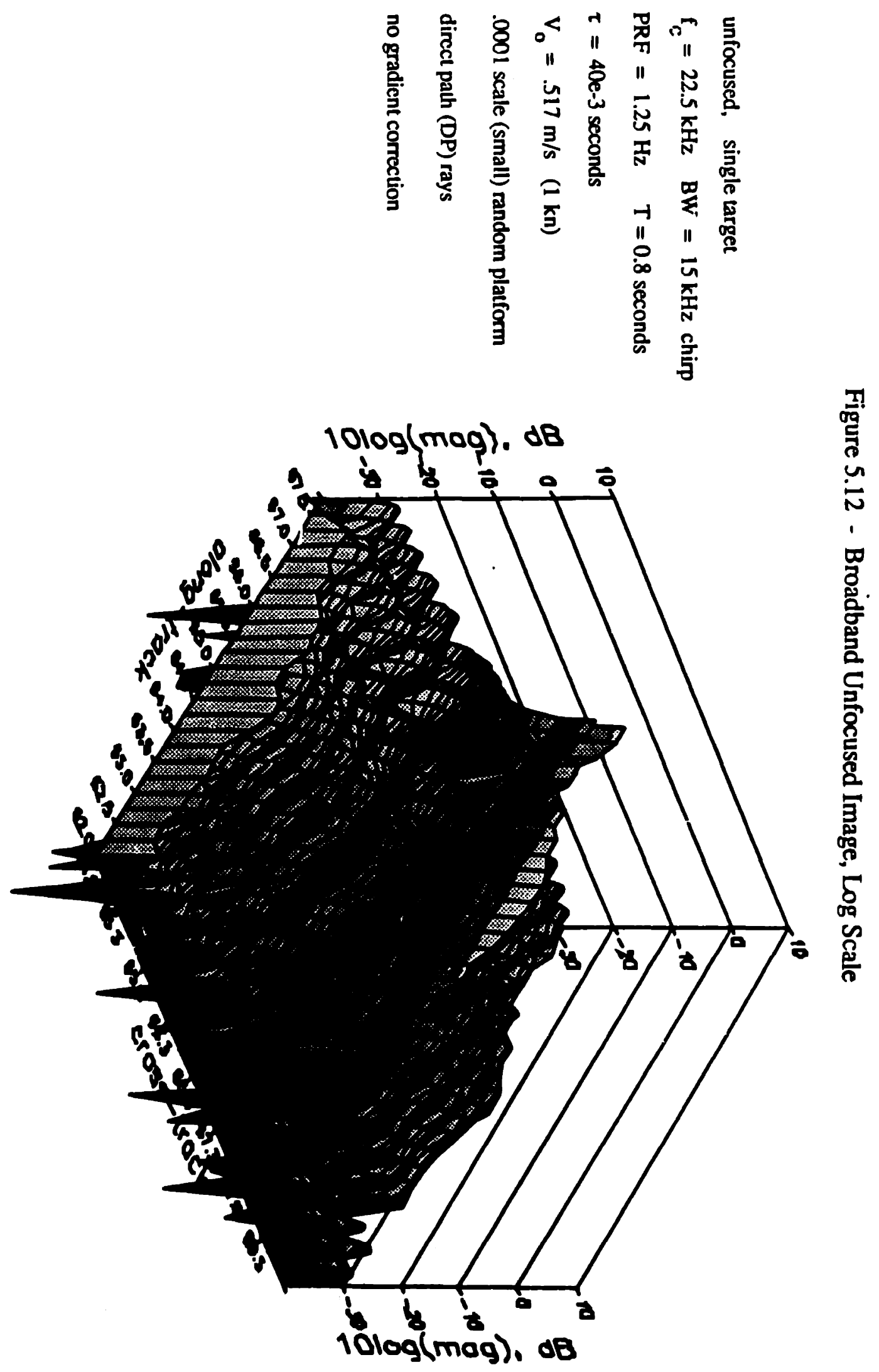




\subsection{Ocean Effects}

"Ocean effects" encompass the influence of an oceanic waveguide. The waveguide has boundaries which may not be smooth, which may be time-varying, which are partly absorptive, and which partly scatter. Multipath always exists in the ocean, although there are circumstances where it has a negligible effect (short range, high frequency operation e.g.). The ucean may also undergo a spatial/temporal change in propagation properties, which for our purposes alters the sound speed profile. These changes may also be the result of internal waves [e.g. Krauss, 1967]. All these features have an influence on the performance of SAS imaging. This section assesses the significance of their influence.

\subsubsection{Multipath}

Two examples are shown to illustrate multipath, using the Gough and Hayes experiment as the framework for the examples. Each example will include the direct path (DP), the surface reflected path (SR), and the bottom-surface reflected (BSR) path. The first case shows a perfectly smooth, perfectly reflecting water surface (relative to the acoustic wavelength), and a perfectly smooth, partially absorptive bottom. The image, shown in Figure 5.13, shows three prominent peaks at the same azimuth (alongtrack), and at different slant ranges; the three peaks correspond to the three paths. The reduced amplitude for the third peak is a consequence of bottom absorption.

The second case, shown in Figure 5.14, randomly perturbs the smoothness of the water and bottom surfaces, which causes the image peaks for the SR and BSR paths to be defocused. The fact that the ocean surface changes with time causes a perturbation in the range coherence from pulse to pulse, leading to a reduction in target amplitude upon azimuthal compression (due to destructive interference). The BSR target image is barely visible in Fig. 5.14, and the SR target image appears to be both reduced in amplitude (compared to the target) and displaced in azimuth. The displacement suggests that the perturbation of the SR echo was not entirely random. This means that the random number generator in the program is more coherent than it should be. However, this example is sufficient to show that perturbations in the bottom and surface multipath echoes are often sufficient to minimize the effects of short-range surface and bottom multipath, especially when the perturbations are large relative to an acoustic wavelength.

This lack of a strong mirror image was observed, in part, in the Gough and Hayes [1989] experiment, where the surface reflection (SR) target image would have been located about 0.5 meters behind the true target image. 


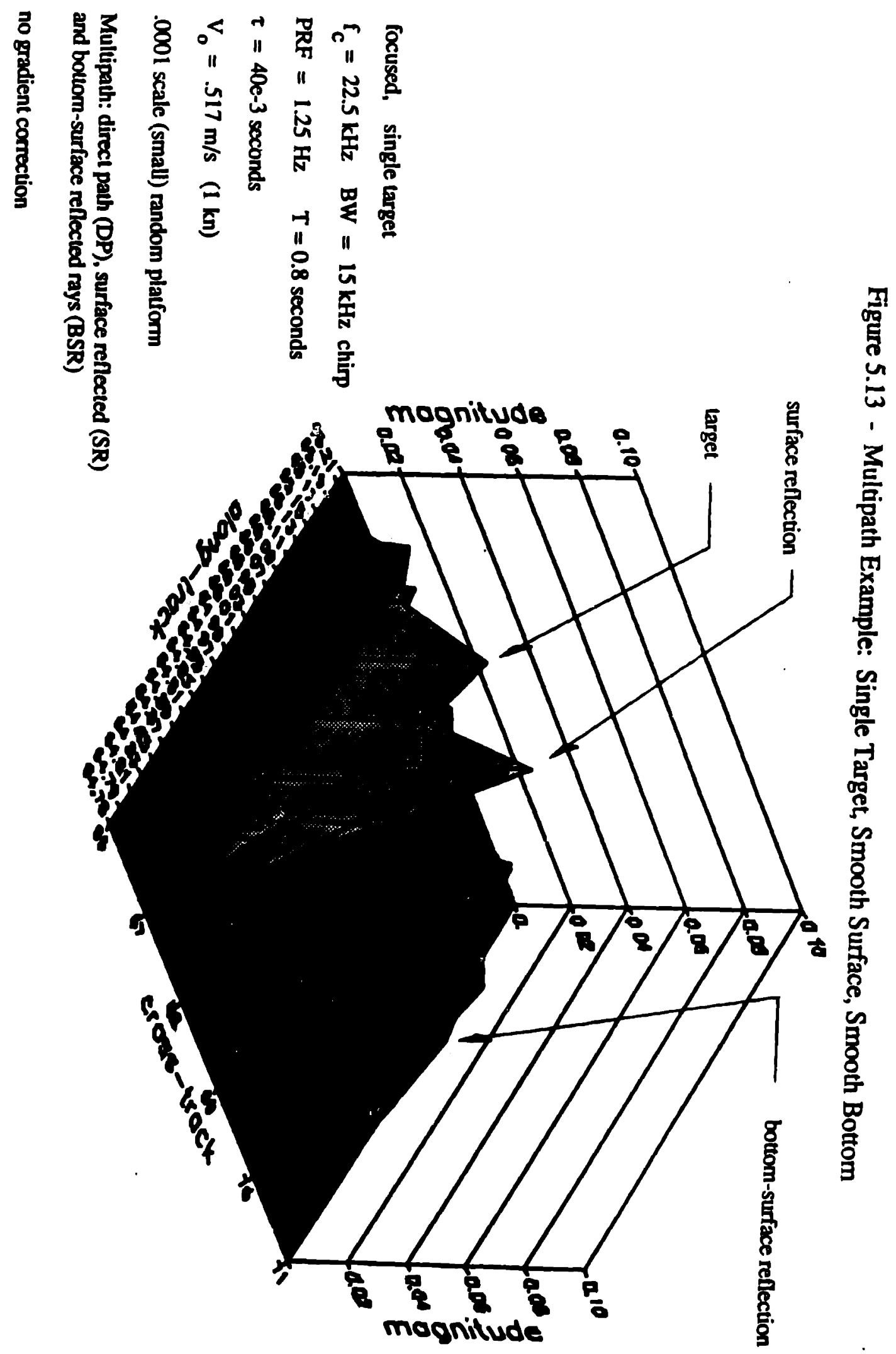




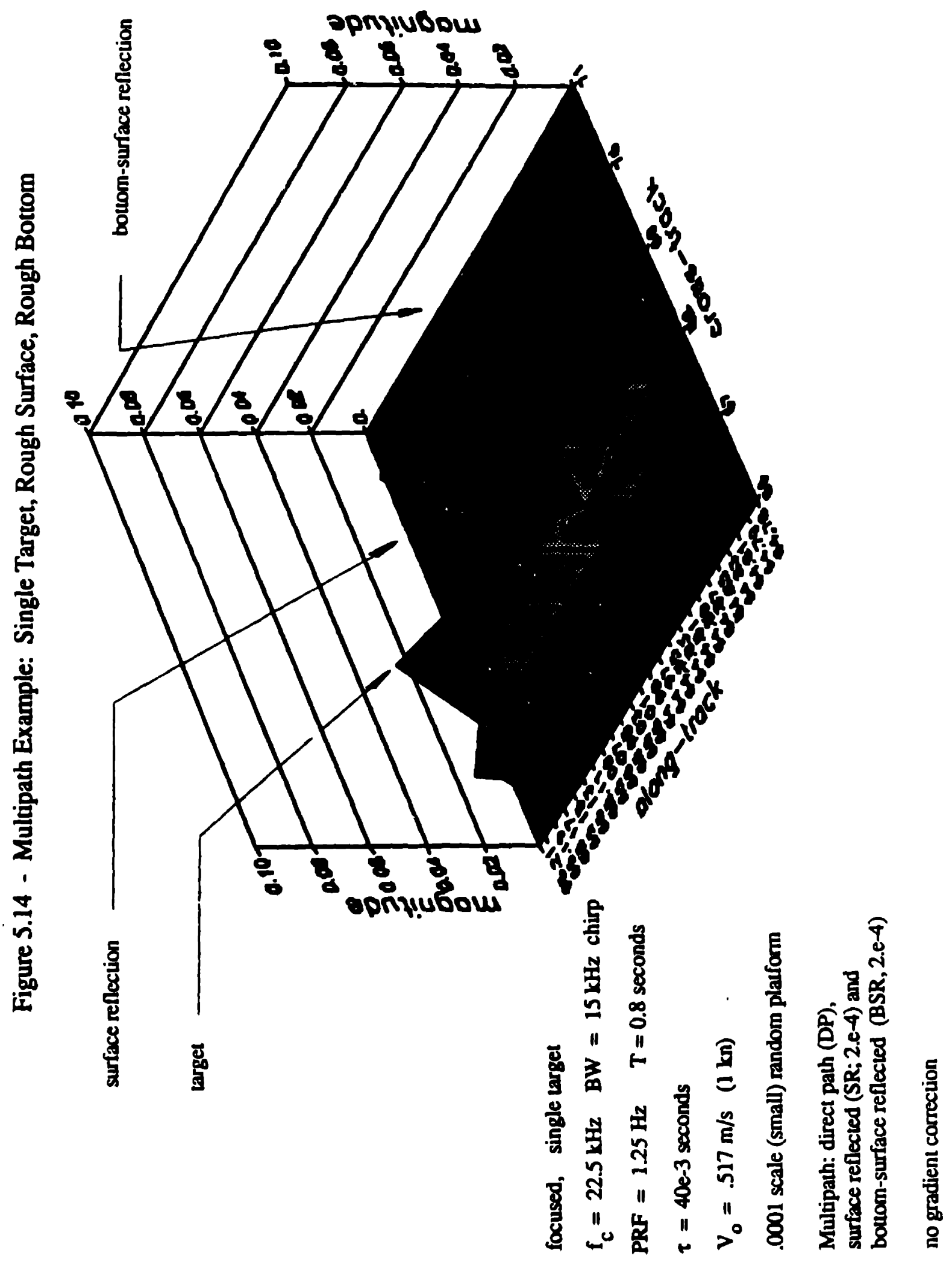




\subsubsection{Deep Ocean Refraction}

The effects of deep ocean refraction on synthetic aperture sonar have been previously studied by Tarng and Yang [1987], although they used a simplified linear profile and showed how refraction over a substantial distance ( $1 \mathrm{~km}$ depth to the target) would slightly alter the along-track resolution. However, the simulations shown by Tamg and Yang are somewhat misleading in the context of synthetic aperture sonar, because the platform speeds used in their paper drastically undersampled the synthetic aperture, leading to a large number of alias target images.

Furthermore, they did not mention the influence of any mismatch between environmental and image reconstruction model parameters, such as the sound speed profile which has a strong influence on image location and resolution, as illustrated in Figure 4.3. The environmental mismatch leads to an incorrect shape of the hyperbolic arc of target range migration, resulting in a degraded image.

Several cases will be shown here to illustrate ocean-model mismatches. The first simulation considers a sonar having the following properties:

Table 5.2 - Deep Océñn Simulation, $30^{\circ}$ Beam, $.10 \mathrm{~m} / \mathrm{s}$

$$
\text { platform: }
$$

$$
V_{0}=.1025 \mathrm{~m} / \mathrm{s} \quad \mathrm{D}=.925 \mathrm{~m} \quad \text { height }=.83 \mathrm{~m} \text { Jepression angle }=-20^{\circ}
$$

transmit:

$$
\begin{array}{ll}
\mathrm{f}_{\mathrm{c}}=3 \mathrm{kHz} & \mathrm{BW}=100 \mathrm{~Hz} \quad \mathrm{~N}=1 \text { (full aperture processed) } \\
\mathrm{PRF}=.222 \mathrm{~Hz} & \tau=.040 \text { seconds }
\end{array}
$$

target:

$$
\text { range } x=2897.5 \mathrm{~m} \quad \text { azimuth } y=850.0 \mathrm{~m} \quad \text { depth }=1002 \mathrm{~m} \text { below sonar }
$$

ocean:

isogradient (1449 at sonar, 1465 at target)

The $-3 \mathrm{~dB}$ mainlobe for this sonar is approximately $30^{\circ}$ (which is slightly more than the $20^{\circ}$ width used by Tarng and Yang.) For a nominal slant range of $3065 \mathrm{~m}$ from the sonar to the target location, the full synthetic aperture length is $1605 \mathrm{~m}$. If a pulse is transmitted once for every platform displacement $\mathrm{D} / 2$, then the total number of pulses is about 3466 . This means that 3466 pulses may be coherently combined to image a single target point, a large computational effort for the computers used to process the data. To reduce the computation time, only a range slice and an azimuth slice are taken, centercd on the 
target location. This gives a reduced image, showing sidelobe structure at the range and along the azimuth of the target, but nowhere else. This is entirely acceptable in view of the representative image in Figure 5.7, which shows that the significant image features near a target point are along the range and azimuth 'ridges' of the target location.

Figure 5.15 shows the azimuth and range image slices for the target location of Table 5.2, as evaluated using a sound speed profile (see example in Appendix D, file: GUESS_PROFILE) which exactly matched the isogradient sound speed profile of the ocean (see e.g. Appendix D, file: PROFILE). The image slices on the right are schematically placed in view on the upper left, and the sound speed profiles appear at the lower left. The target appears in the correct azimuth-range location, and the azimuth and range resolutions are respectively .73 and 4.2 meters (.46 and 6.9 meters per theory).

Figure 5.16 shows azimuth and range images for the same sonar data when the reconstruction profile was isospeed (i.e. the sound speed was constant; see bottom left illustration for profiles). The location of the range maximum was chosen as the location for the azimuth image slice. The extreme mismatch between the ocean and the reconstruction model sound speed profiles resulted in a target which was apparently split along azimuth, resulting in an ambiguous image. The amplitude of the image maxima are also significantly reduced from the image in Fig. 5.15 (.0016 strong mismatch vs, .006 for match). When viewed along the range axis, the image maximum is found to be displaced from the true target range (2880 m. strong mismatch vs. $2897 \mathrm{~m}$. true). When displaced along azimuth (symmetrical either to the left or right), the image reaches a maximum in two locations. This illustrates the effect of a mismatched sound speed profile when reconstructing a synthetic aperture image of a point target: the target peak is smeared into lobe-like structures resembling sidelobes, and the amplitude of the target image is strongly reduced. The mismatch in sound speed during image reconstruction leads to an incorrect hyperbolic range migration arc to be used, and this effectively wipes out the target image.

Of course, if even a crude approximation to the ocean isogradient profile were used in the reconstruction, the image is preserved nearly intact, as shown in Figure 5.17. In this case, the model profile takes an approximate estimate at the ocean profile (left illustration). Note that the target is correctly located, the azimuth and range resolutions are not substantially different from Fig. 5.15, and the amplitude of the image maximum is about .003 (versus .006 in Fig. 5.15, match profile). 

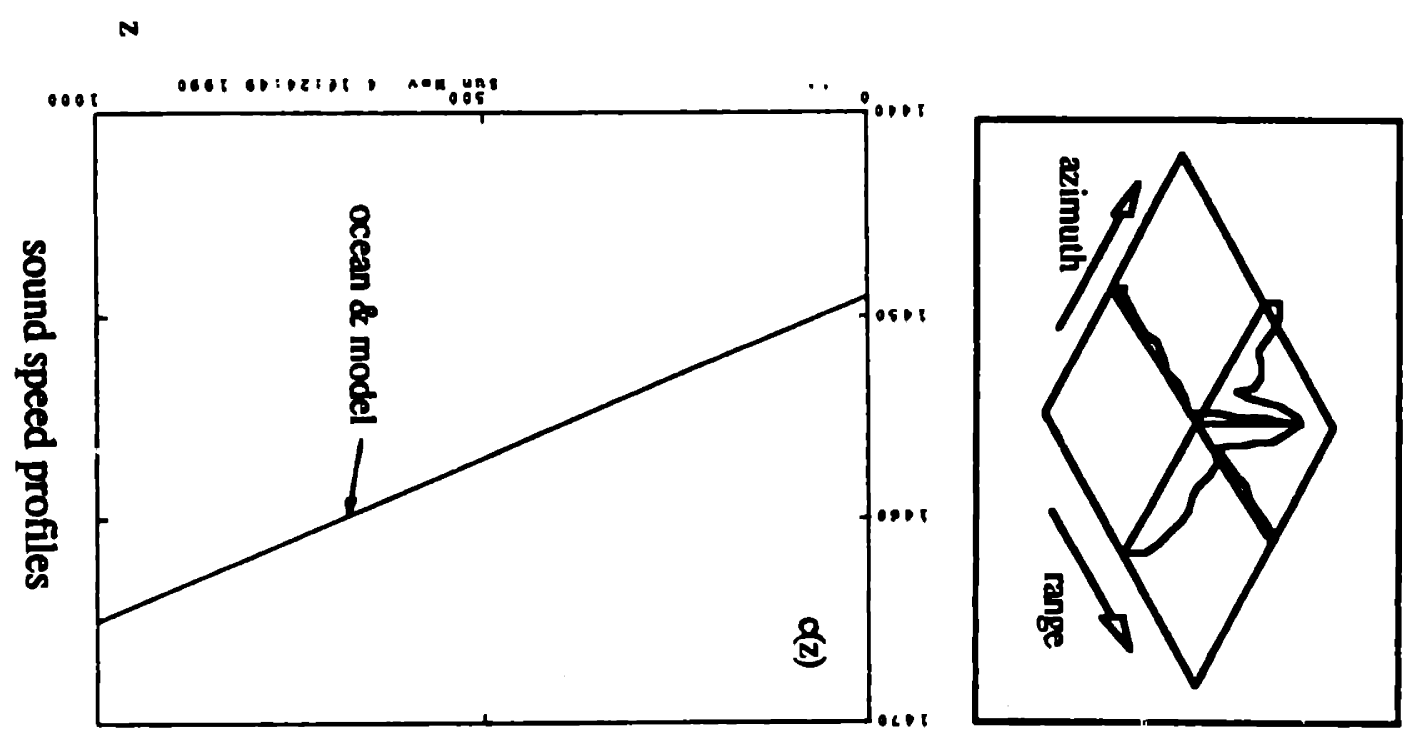

里

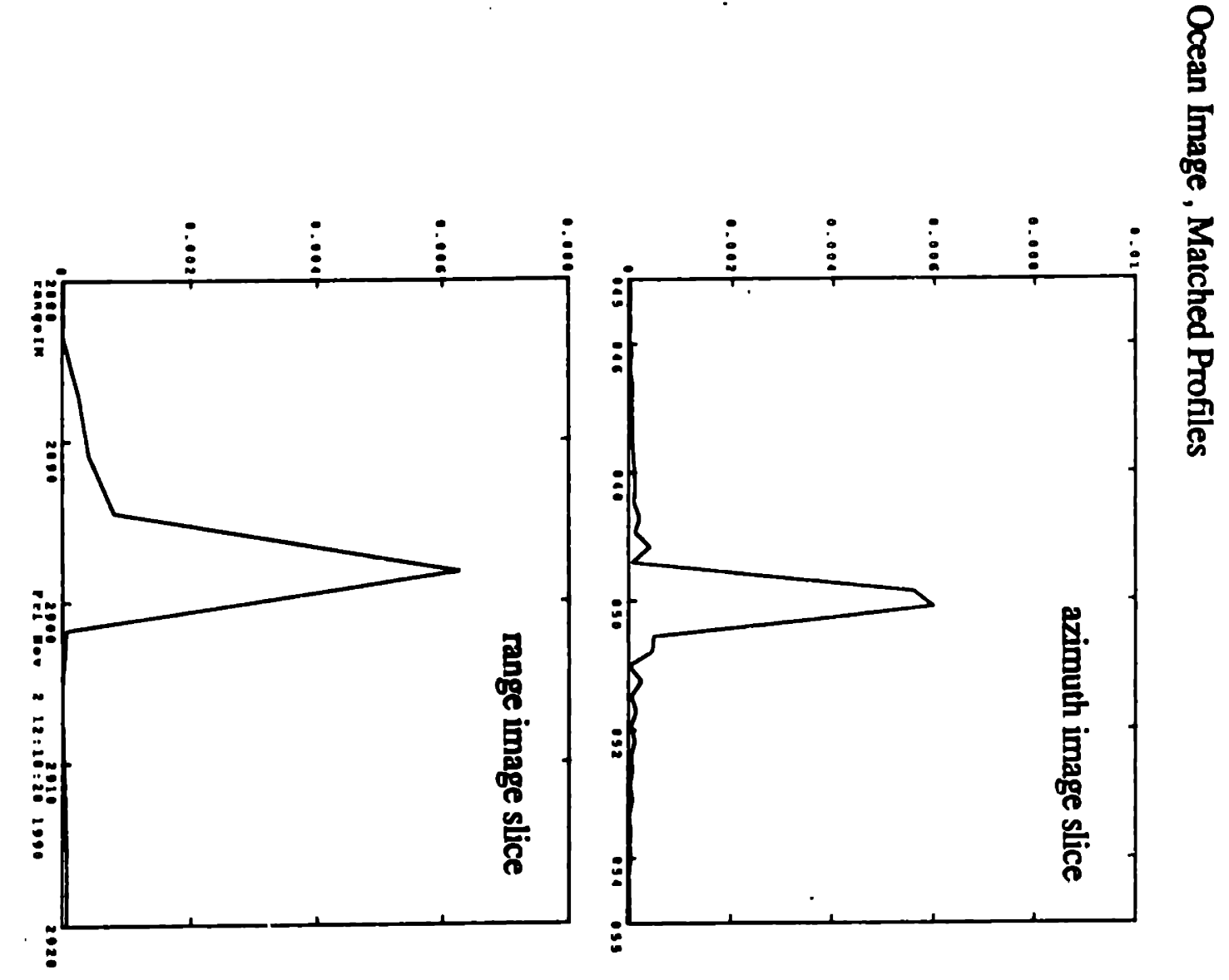




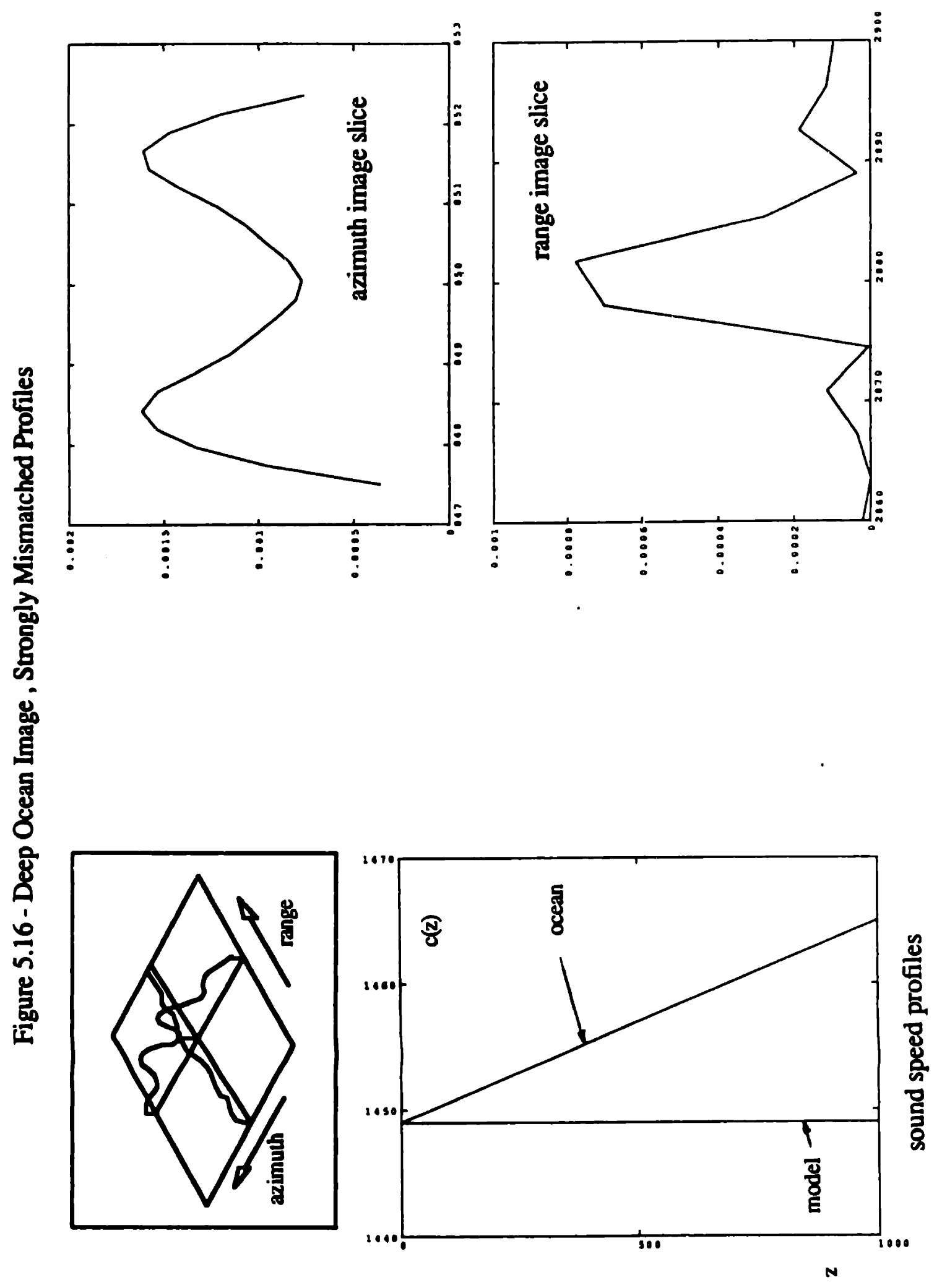




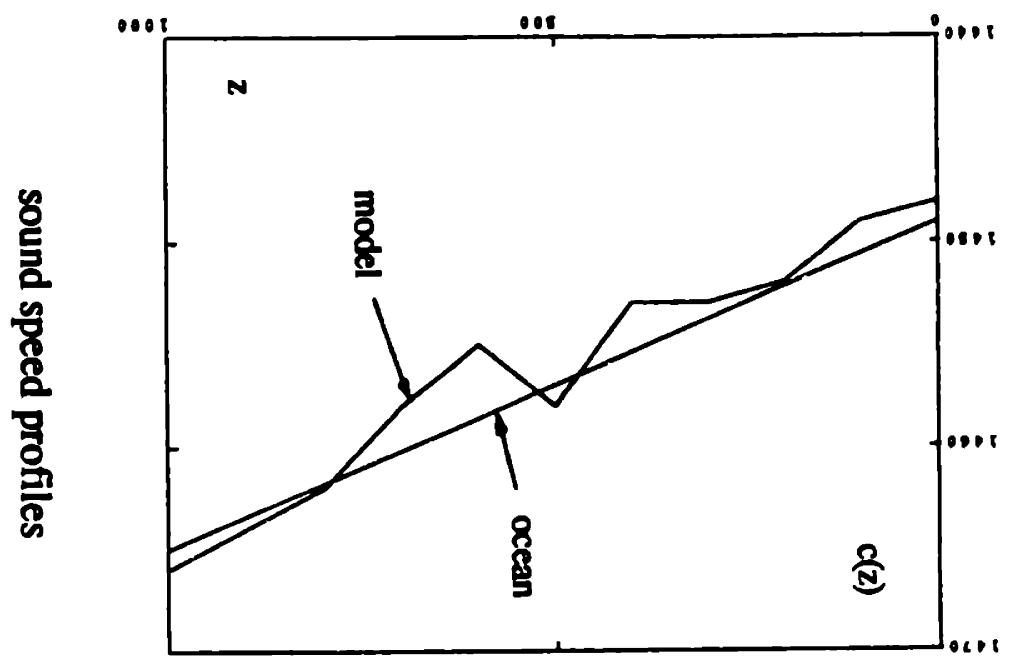

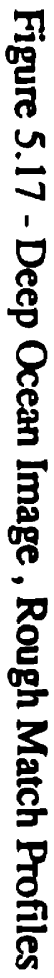
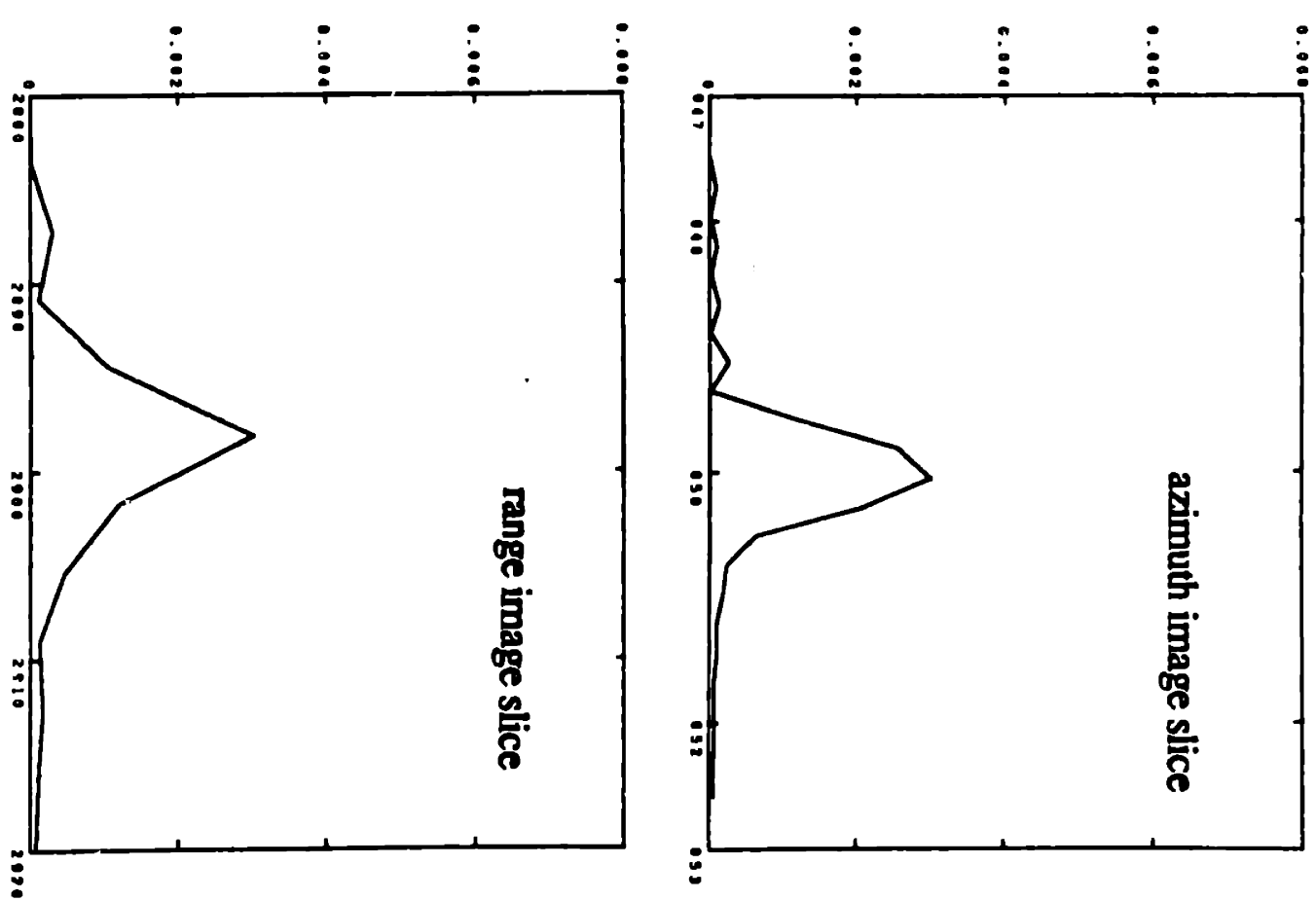

163 
Another way to reduce the influence of ocean-model environmental mismatch is by reducing the width of the sonar platform mainlobe. Recalling Figure 4.3, in the neighborhood of the broadside slant range $R_{0}$ the isogradient and isospeed arcs have similar curvature, which means that the range migration arc match between ocean and reconstruction model is better for narrower beam sonars. A narrower (real) beam then reduces the length of the synthesized aperture, which of course gives poorer resolution, but also reduces the computation needed to form images, and reduces influence of the medium coherence time.

As an example, we alter the sonar characteristics of Table 5.2 to those shown in Table 5.3 (faster platform, closer target).

Table 5.3 - Deep Ocean Simulation, $5^{\circ}$ Beam, $.55 \mathrm{~m} / \mathrm{s}$

\begin{abstract}
platform:$$
V_{0}=.555 \mathrm{~m} / \mathrm{s} \quad D=5 \mathrm{~m} \quad \text { height }=.83 \mathrm{~m} \quad \text { depression angle }=-20^{\circ}
$$

transmit:$$
\mathrm{f}_{\mathrm{c}}=3 \mathrm{kHz} \quad \mathrm{BW}=100 \mathrm{~Hz} \quad \mathrm{~N}=1 \text { (full aperture processed) }
$$$$
\mathrm{PRF}=.222 \mathrm{~Hz} \quad \tau=.040 \text { seconds }
$$

target:

$$
x=2897.5 \mathrm{~m} \quad \mathrm{y}=170.0 \mathrm{~m} \quad \text { depth }=1002 \mathrm{~m} \text { below sonar }
$$

ocean:

isogradient (1449 at sonar, 1465 at target)

Since the frequency is the same as the previous cases, the sonar transceiver becomes longer, reducing the beamwidth. This in turn reduces our need to transmit as frequently, since we need only transmit every $\mathrm{D} / 2$, and so the $\mathrm{PRF}$ is reduced. Figure 5.18 shows, side by side, a matched-profile image (left, isograntient model) with a mismatched profile (right, isospeed model). The matched example has an image amplitude of $2.2 \times 10^{-4}$ and is correctly located in azimuth and range. The mismatched example has an image amplitude of $1.8 \times 10^{-4}$, is displaced in range by about -20 meters, and appears to be spread over the range axis more so than the matched example. However, in spite of the differences in amplitude and location, the image is nonetheless preserved because the mismatch in sound speed profiles has a small influence on
\end{abstract} coherent imaging. 

One last look at the influences of deep ocean refraction is a sevies of images for different profile mismavshes from an isogradient, narrow beam sonar. Several parameters were changed in these examples: the target location (and therefore the sonar depression angle), and therefore the processing demands were altered due to the closer target $\left(P R F=.555 \mathrm{~Hz}, V_{0}=1.38 \mathrm{~m} / \mathrm{s}\right)$, as listed in Table 5.4 .

Table 5.4 - Deep Ocean Simulation, $5^{\circ}$ Beam, $1.38 \mathrm{~m} / \mathrm{s}$

$\begin{array}{llll}\text { platform: } & & \\ & V_{0}=1.38 \mathrm{~m} / \mathrm{s} & \mathrm{D}=5 \mathrm{~m} & \text { height }=.83 \mathrm{~m} \quad \text { depression angl } \\ \text { transmit: } & & \mathrm{BW}=100 \mathrm{~Hz} \quad \mathrm{~N}=1 \text { (full aperture processed) } \\ & \mathrm{f}_{\mathrm{c}}=3 \mathrm{kHz} & \mathrm{PRF}=.555 \mathrm{~Hz} & \tau=.040 \text { seconds }\end{array}$

target:

$$
x=2897.5 \mathrm{~m} \quad y=140.0 \mathrm{~m} \quad \text { depth }=1002 \mathrm{~m} \text { below sonar }
$$

ocean:

isogradient (1449 at sonar, 1465 at target)

Of the cases studied, none were significanly different from the rest, so only five examples are shown among the three Figures, 5.19 through 5.21, accompanied by their sound speed profiles (ocean vs. model). In synopsis, a reasonably close guess at the sound speed profile will image the target, and locate it with some cerainty. An unreasonable guess will slighuly defocus the image, and shift its location either closer or further than the true location. When all else fails; a isospeed estimate for the sound speed will generally give good results. The basic insensitivity of ocean-model mismatch for image formation occurs because no matter what sound speed profile (i.e. the model) is used during image reconstruction, the elapsed time (ET) ray path from broadside is linearly related to the ET ray path at the $-3 \mathrm{~dB}$ sonar beam extreme, in the same way that a leg of a right triangle is linearly (or geometrically) related to the length of the hypotenuse, so that virtually any profile will work. This is true because the sonar mainlobe is fairly narrow to begin with. Previous examples in this section showed the defocusing results for a deep ocean $30^{\circ}$ wide sonar beam, which does not have ray path ETs that are linearly related.

This is a restatement, in words, of the illustration in Figure 4.3: for narrow beam sonars, the ocean hyperbolic arc (shown as isogradient) is essentially the same as the model (shown as isospeed). For wide angle sonars, the hyperbolic arcs are not similar, and this is observed as a defocusing or blurring of the image. 
Figure 5.19 - Deep Ocean Image, Narrow Sonar Beam, Mismatch Series
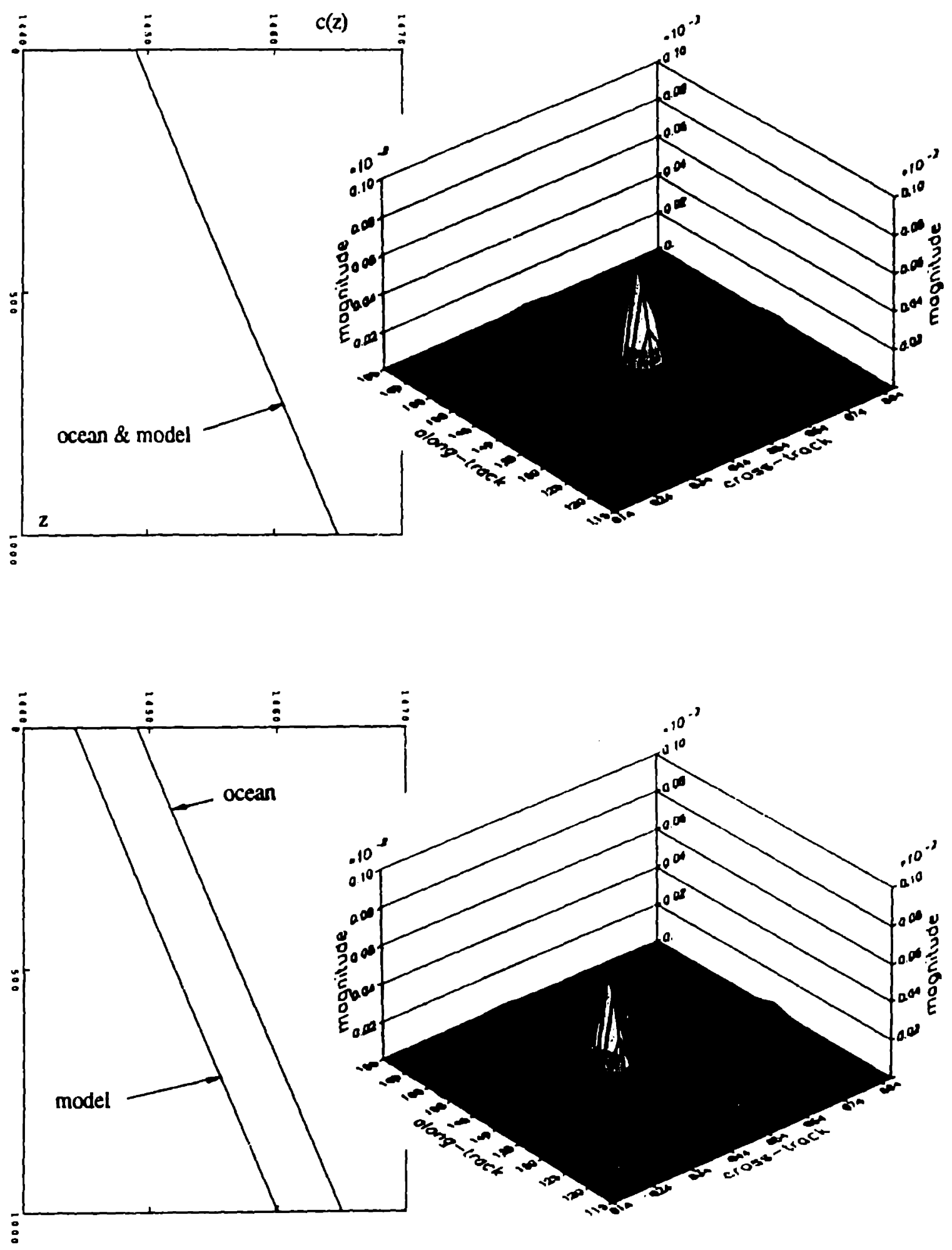
Figure 5.20 - Deep Ocean Image , Narrow Sonar Beam, Mismatch Series Continued
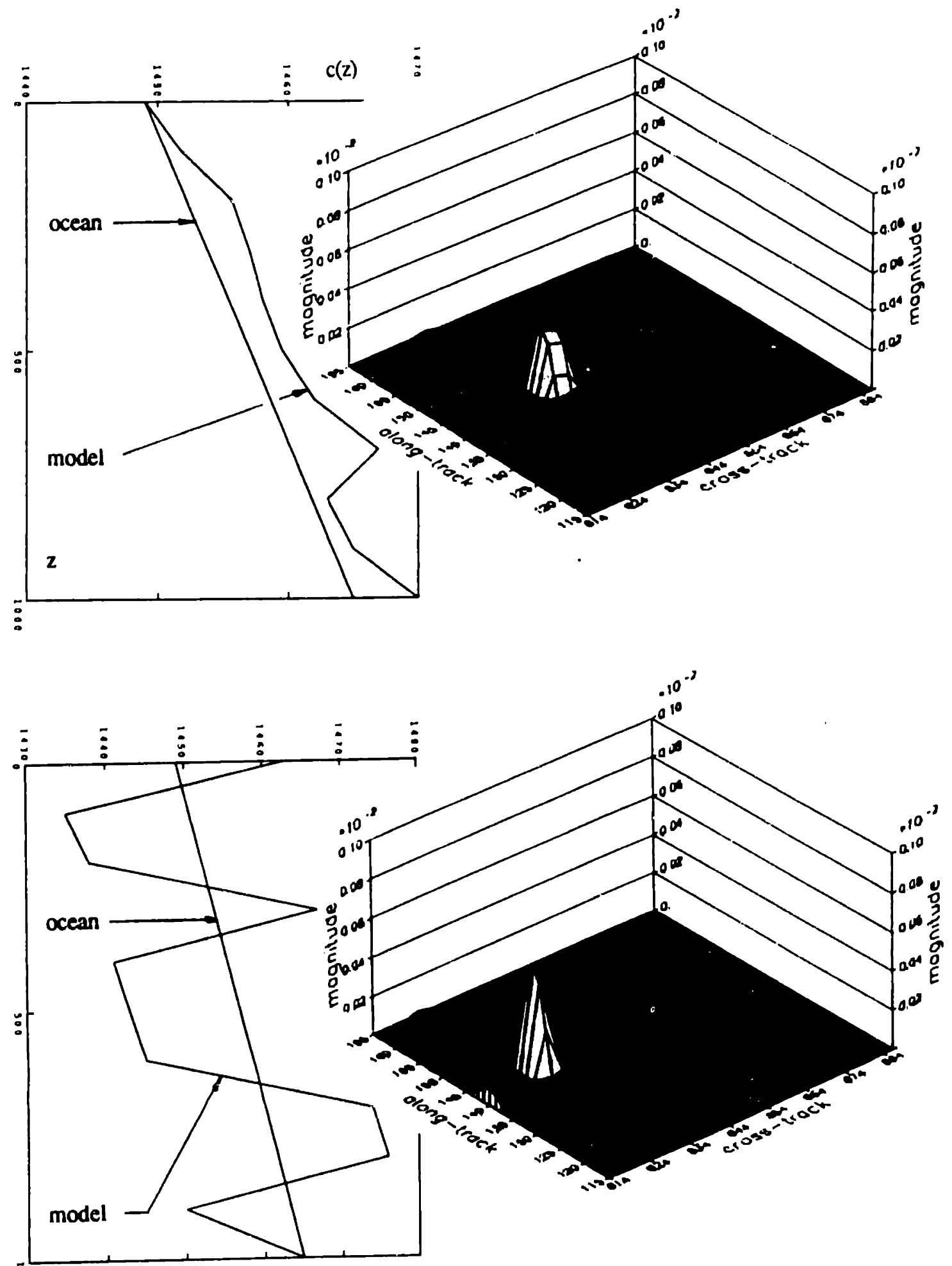
Figure 5.21 - Deep Ocean Image , Narrow Sonar Beam, Mismatch Series Continued

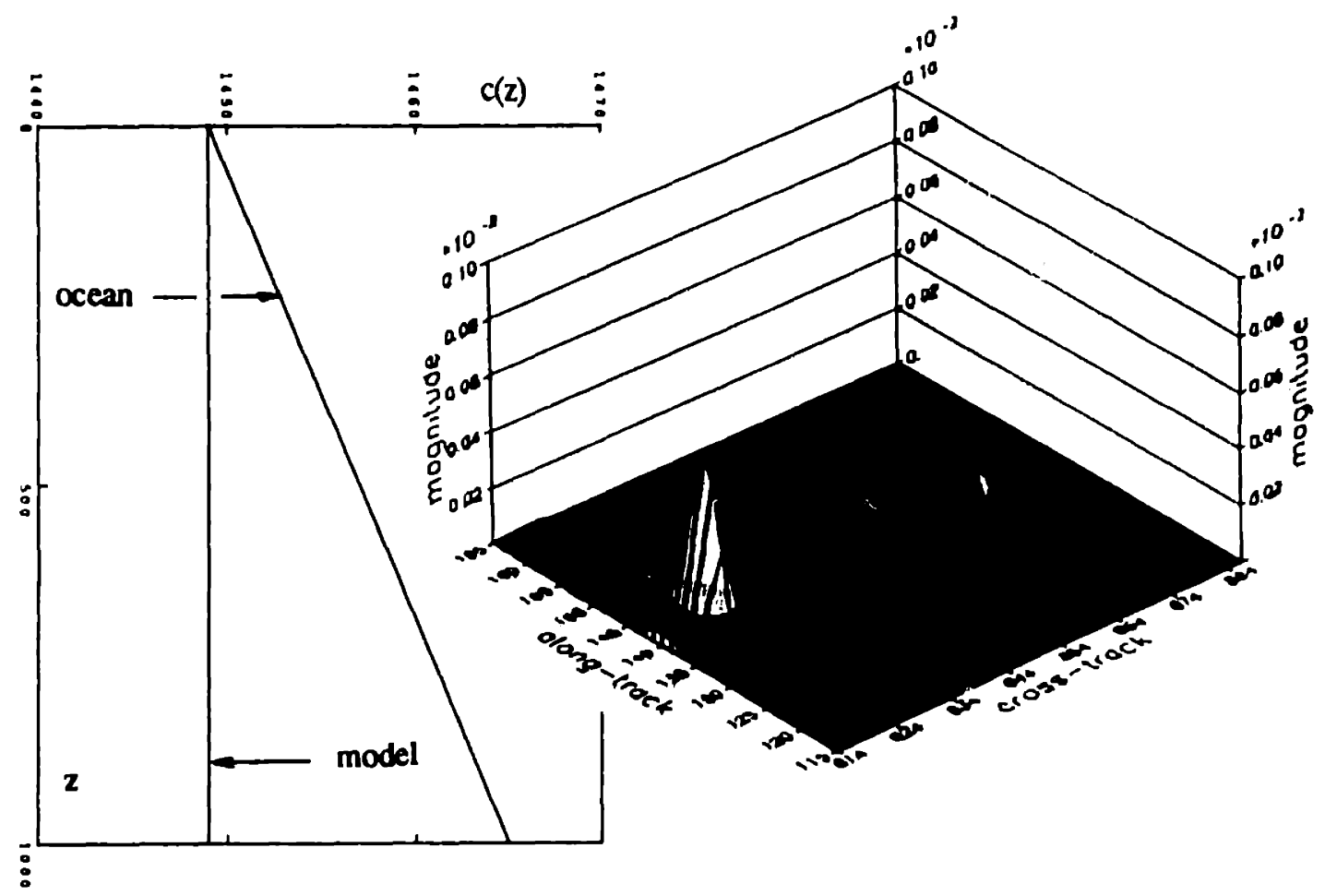




\subsubsection{Coherence Time}

The stability of the acoustic medium, which was discussed and the experiments reviewed in Chapter 4 , is described in terms of its spatial coherence and temporal coherence. Though spatial and temporal coherence are not necessarily linked, for the purpose of the computer model used here, I have assumed that they are. The medium has therefore, a spatial coherence which I have linked to the temporal coherence via the platform speed (see section 4.2). The examples shown here use the focused Gough and Hayes experiment as the framework to illustrate the influence of coherence time.

For example, we compare two SAS images of the Gough and Hayes experiment, as shown in Figure 5.22. The image on the left has a medium temporal coherence time of 120 seconds ( $100 \mathrm{~ms}$ angle). In order to take full advantage of all target echoes received in the main sonar beam, the coherence time needed to be at least 29 seconds. The image shown on the right in Fig. 5.22 has a coherence time of 14 seconds, so only about half of the echoes will add constructively during image processing. Indeed the one-half estimate was the case since the magnitudes of the target images were respectively .16 and .075 for the left and right images. The resolutions in azimuth and range were respectively: $.16 \mathrm{~m}$ and $6 \mathrm{~cm}$ on the left (120 sec. image), and $.19 \mathrm{~m}$ and $6 \mathrm{~cm}$ on the right (14 sec. image).

The comparison shown by this example is more or less obvious by inspection. If we allow data to be collected for a longer time for a target location, we known thai the synthetic aperture which may be formed is longer and hence the azimuth resolution is smaller (better). However, if we collect data over a period of time which exceeds the coherence time ( $\mathrm{T}_{\text {cohere, }}$ with an associated variance $\sigma_{\mathrm{rms}}$ ) then upon image formation, some of the data will not add constructively during the azimuth compression and this will lead to a smearing of the target image. In the limit, if we continue to take data for any time exceeding the coherence time (from the time we started collecting data for the target) then the destructive interfercnce in azimuthal compression will reduce the target image to obscurity in the 'background noise' of the image. For the example shown in Fig. 5.22, we observed: a smearing of the target image in azimuth (though there was no measurable smear in range), a reduction in scattering amplitude (i.e. the image magnitude), and an increase in the azimuthal sidelobes.

Of course the benefit of synthetic aperture processing is that the image may always be reconstructed from the raw echo data using different amounts of the aperture, and hence different dwell times. This allows the several images to be formed, from which the best tradeoff of resolution versus noise may be detcrmined. 

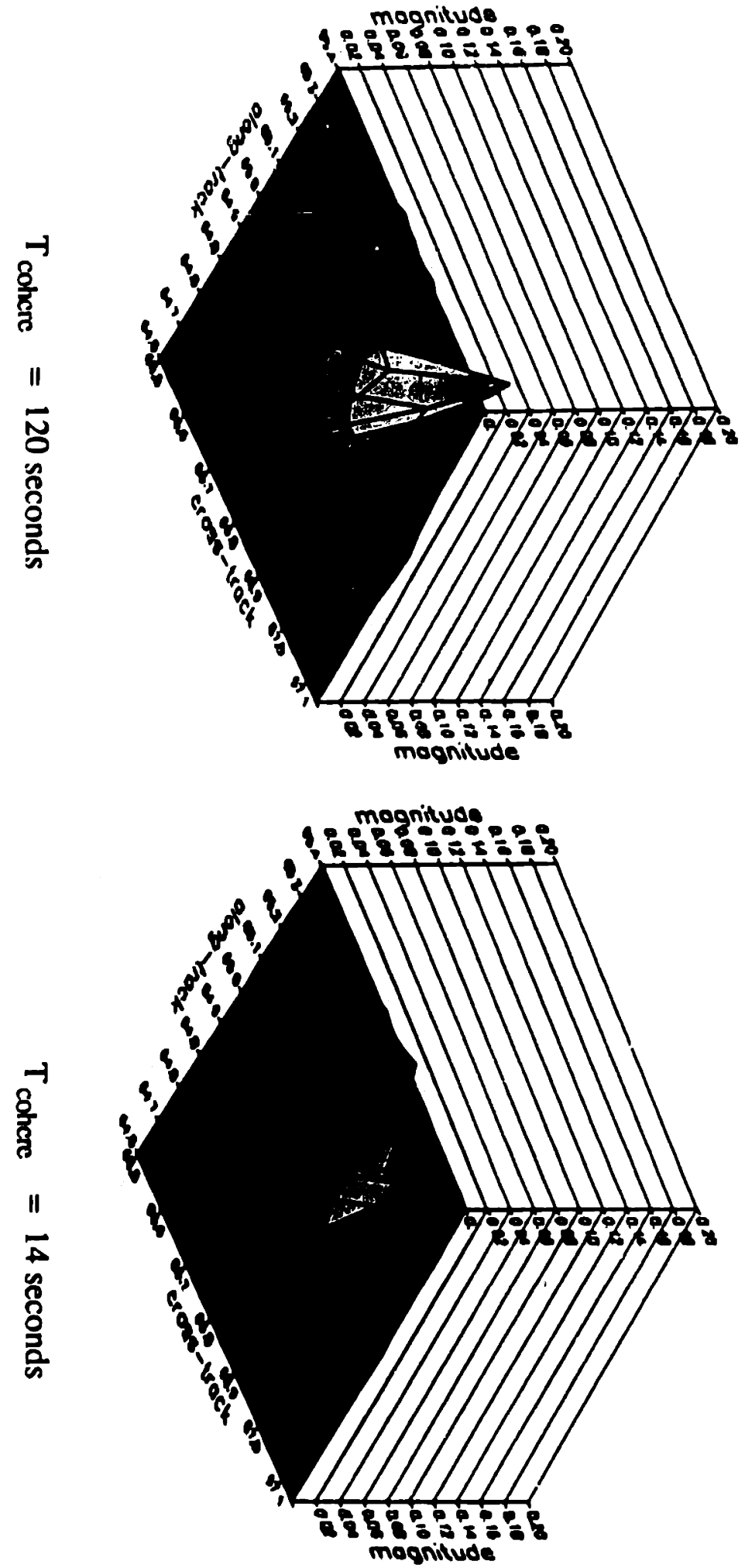

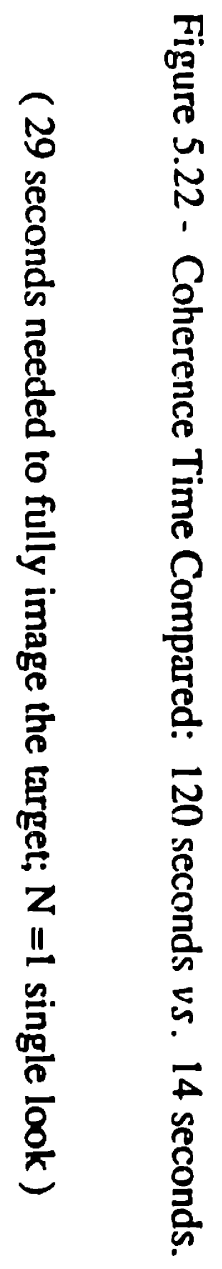




\subsection{Platform Efrects}

Two platform effects are shown. The first is the effect of a mismatched platform speed in the image reconstruction. The second is the effect of uncompensated platform motion during data collection, and how it influences the image.

\subsubsection{Mismatch Along-Track Platform Speed}

Hayes [1989] has shown a few examples of the mismatch of the actual platform speed (from the Gough and Hayes [1989] ) during data collection with the one used in the image reconstruction (i.e. in the image processing). Two examples are shown here for respective comparison with Figure 7.8, parts (a) and (c) in Hayes' 1989 thesis. These two examples are shown in Figure 5.23. The first (on the left) shows an image having a reconstruction platform speed $(.70 \mathrm{~m} / \mathrm{s})$ which matches the experiment speed; the secona (on the right) shows an image having a reconstruction platform speed of $.73 \mathrm{~m} / \mathrm{s}$ which slightly exceeds the experiment speed. Note that the image amplitude is reduced from .115 in the matched case to .061 for the mismatched case. Accompanying the loss in target amplitude is an increase in the apparent sidelobe level, and a azimuthal displacement of the target image from $65 \mathrm{~m}$ to about $66.6 \mathrm{~m}$.

Fiayes showed images for several other image reconstruction platform speeds both above and below the true platiorm speed, but they are not recreated here. Figure 5.23 is sufficient to confirm the results of Hayes, and to show that the platform speed must be known with some confidence for sharp imagery.

Effects similar to those shown here may also be found by using a mismatched value of the PRF, because the spacing of each element of the synthetic array is determined by $D_{\text {pulso }}=V_{0} / P R F$. Thus, a PRF which is too small during the reconstruction will have the same effect on imaging as a $V_{0}$ which is too large. 


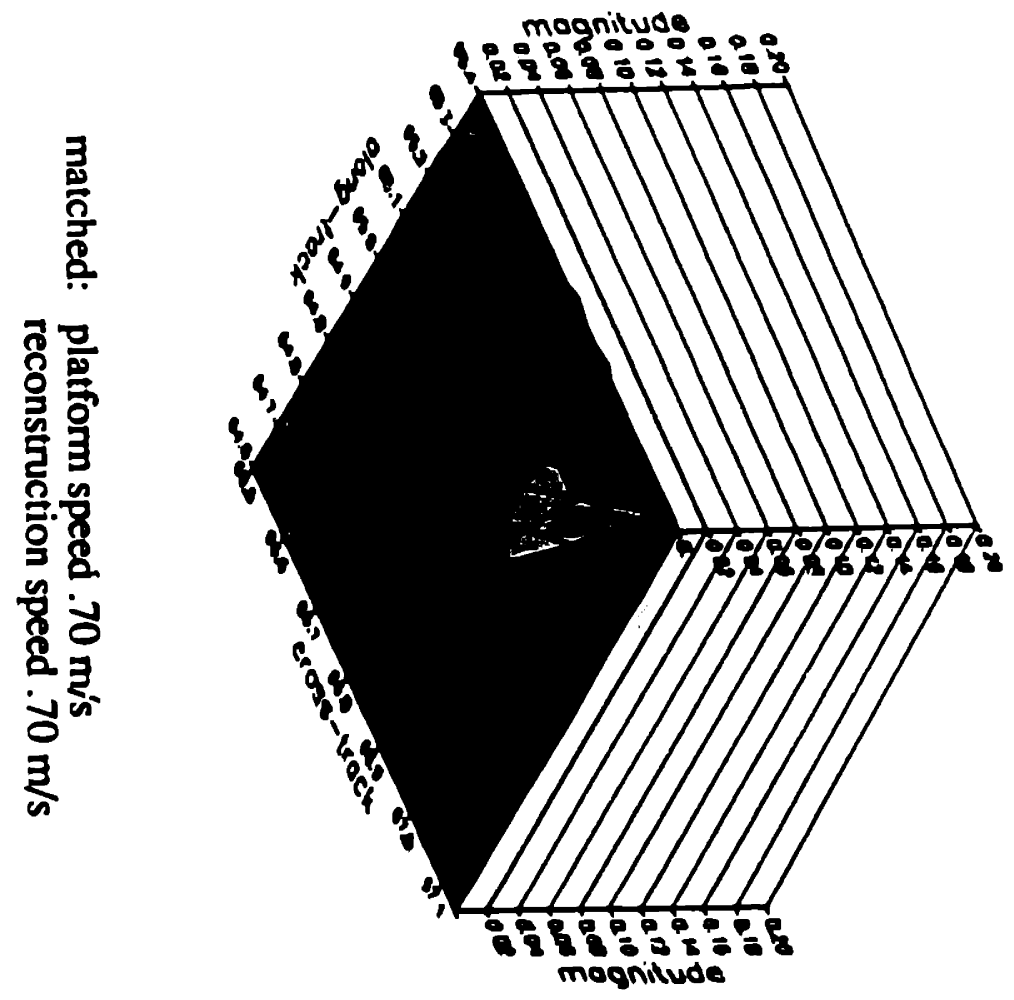

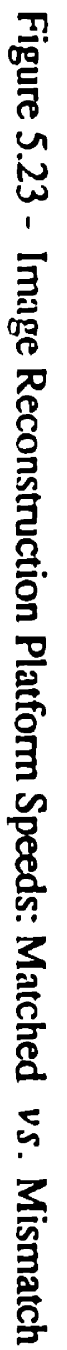




\subsubsection{Uncompensated Platform Motion}

All the images shown thus far have made the basic assumption that the platform location, velocity, and acceleration were known for all transmit-receive stations along a synthesized aperture. Equivalently, this means that the platform motion was either 'perfect' (as though it were guided along smooth rails) or that the motion was appropriately compensated in each received time series. This section departs from this assumption and deliberately adds elapsed time errors to the received echoes, which in turn mimics a platform position error during transit. The examples shown in this section show random platform motion superposed along a straight path; sinusoidal motion superposed along a straight track may also be implemented although the results are not explicidy shown here. Several contrasting figures are shown, but most of the results are summarized in two tables. As in the previous section, the Gough and Hayes [1989] experiment will be used as the framework model to illustrate the effects of uncompensated platform motion on imagery.

Platform motion is induced in the model by perturbation of the elapsed time of flights for each successive echo from the target. A random number is generated by the model between the values 0.5 and -0.5. This random number is then multiplied by a user-set scale factor, the average wavelength, and the result is divided by a nominal sound speed (i.e. $1500 \mathrm{~m} / \mathrm{s}$ ). This process gives a random time error to the $\mathrm{n}$ th pulse, and the random error is assumed to be constant over the interpulse time (this is not strictly true for a real ocean environment, but it is a reasonable estimate when imaging a single target). The $+/-$ nature of the random number (having an estimated zero mean), combined with the scale factor, gives a platform motion envelope. The statistics of the motion were not calculated (although they could be), because if the platform motion were capable of being measured, then the error from a straighuline track (and crror in Doppler if significant) could be removed from the time measurements. In this case, we are 'unable' to measure the platform motion, so we instead set an envelope bound on the platform motion and look for degradation in the images as the platform motion envelope becomes larger.

Two random platform motion examples are shown in Figure 5.24 (logarithmic). The left-most image has a $\lambda_{\mathbf{a v g}} / 16$ scale envelope, which corresponds to a worst-case $\lambda_{\mathrm{avg}} / 82$-way scale error, which is considered small. The target has an image amplitude of .149 (a near motion-free valuc), is correctly located, and the resolutions are 17 and $5 \mathrm{~cm}$ respectively for azimuth and range. In contrast on the right is an image for a $2 \lambda_{\text {avg }}$ envelope (worst-case $4 \lambda_{\text {avg }} 2$-way scale error). This image has an amplitude of .034 , is ambiguously located (see nearby false target), and has respective resolutions which are not easily measured due to the multiple image lobes. 


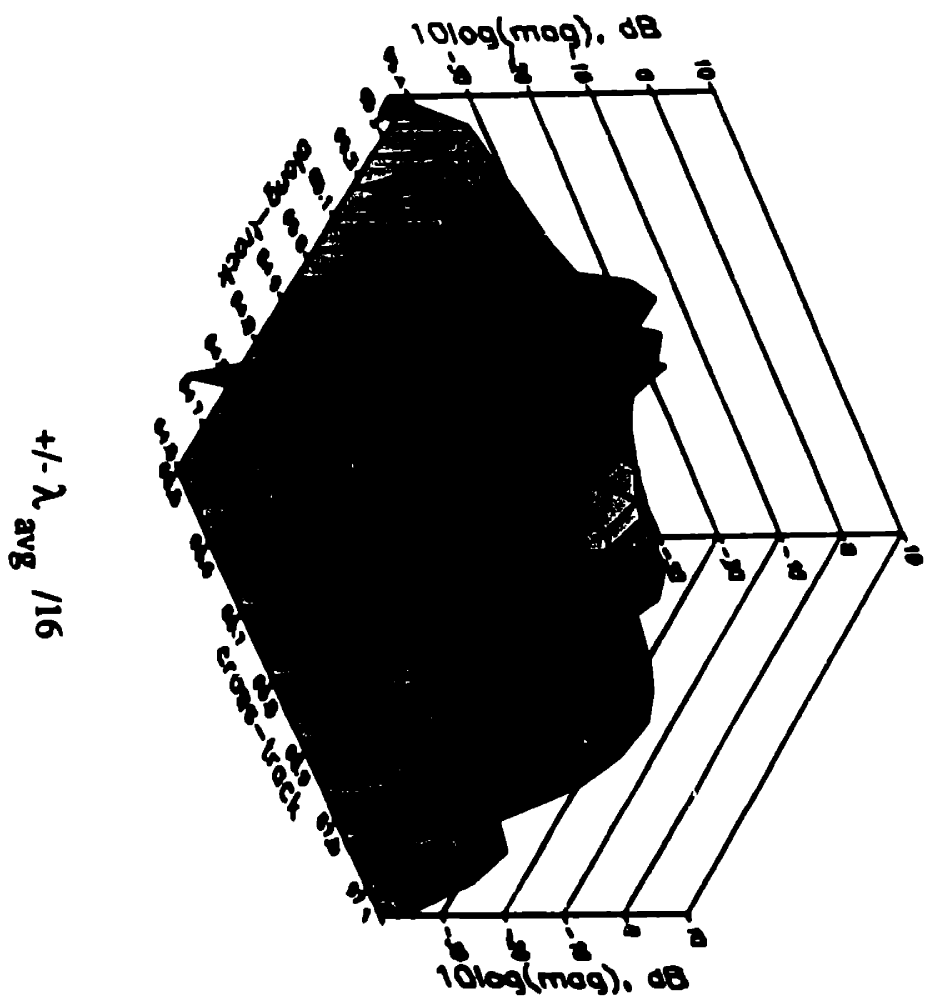

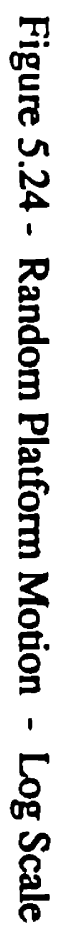

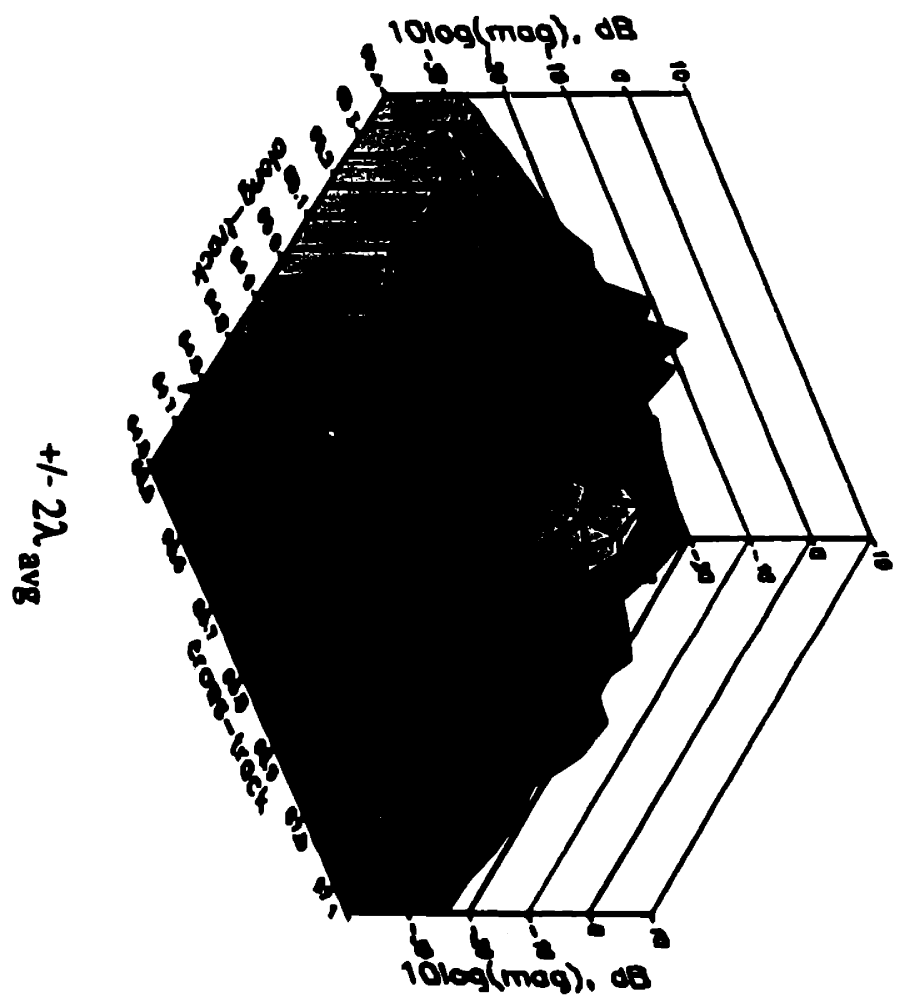


The sidelobe comparison is difficult to make because the right-most image has sidelobe-like structures, but not necessarily in the same range-plane or azimuth-plane as the target location. It appears that the sidelobes have changed from about $-9 \mathrm{~dB}$ in the left image, $10-7 \mathrm{~dB}$ in the right. The image on the left is clearly superior (as it should be); the difference in image quality is even more striking in Figure 5.25 which shows the same two images but now in a linear scale.

The intermediate examples between the two shown in Figures 5.24 and 5.25 are not furnished here, but the relevant information from them is summarized in Table $5.5 \ldots$

Table 5.5 - Random Platform Motion Examples

\begin{tabular}{cccccc}
$\begin{array}{c}\text { envelope } \\
+/-\end{array}$ & scale & image max. value & $\begin{array}{c}\text { azimuth sidelobe } \\
(\mathrm{dB})\end{array}$ & $\begin{array}{c}\text { resolution }(\mathrm{cm}) \\
\text { range azimuth }\end{array}$ \\
\hline$\lambda / 16$ & $1 / 16$ & .149 & -9 & 17 & 4 \\
$\lambda / 8$ & $1 / 8$ & .334 & -9 & 18 & 4 \\
$\lambda / 4$ & $1 / 4$ & .092 & -9 & 20 & 5 \\
$\lambda / 2$ & $1 / 2$ & .073 & -9 & 15 & 3 \\
$\lambda$ & 1 & .058 & -7 & 23 & 6 \\
$2 \lambda$ & 2 & .034 & -7 & $?$ & $>10$
\end{tabular}

a. range sidelobes not found due to choice of image area. 


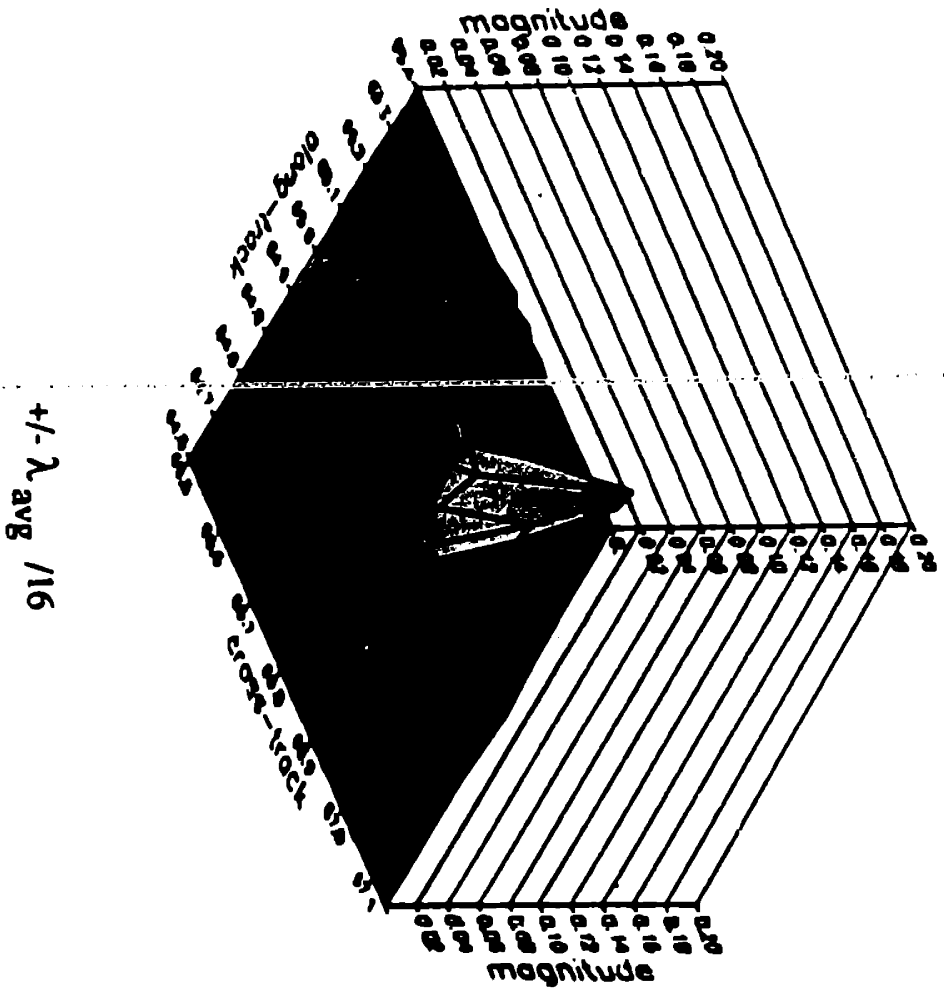

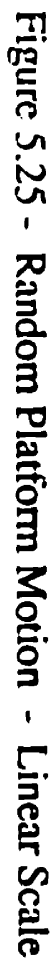

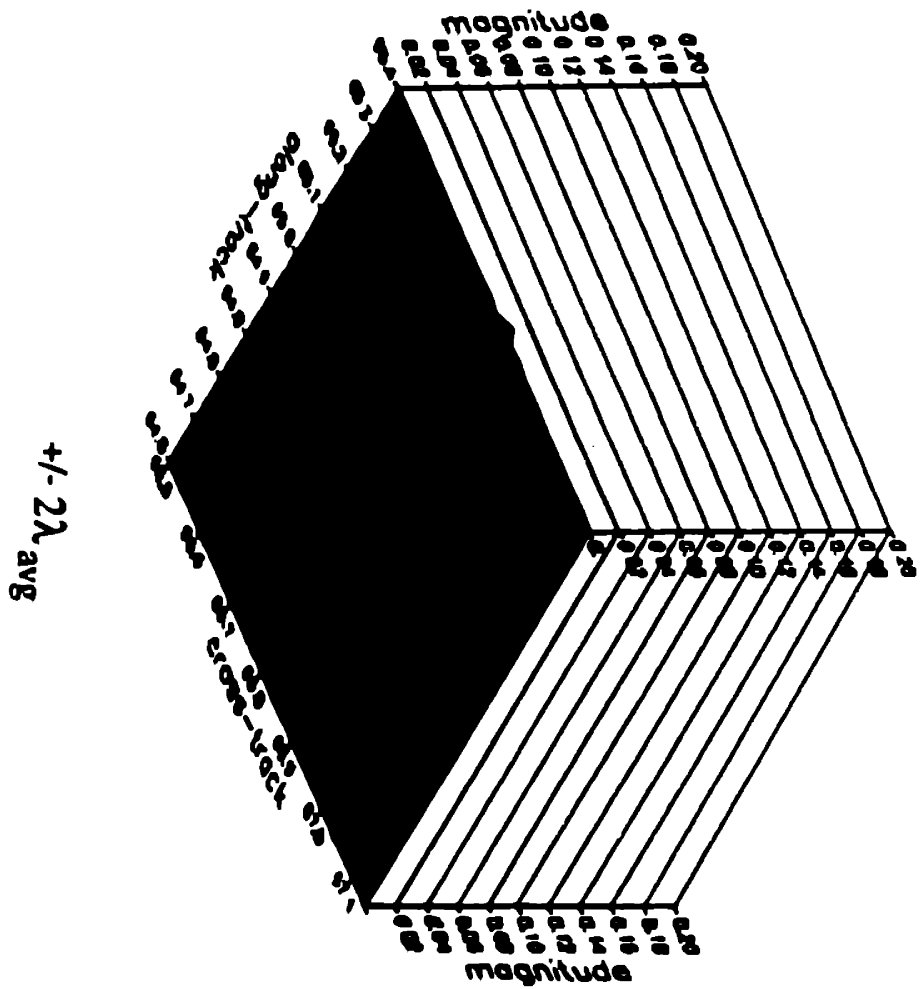


The sinusoidal option available for platform motion allows several platform influences to be studied. A very large period (very low frequency platform oscillation) combined with a large scale factor leads to a platform track which is skewed from the intended one. This could occur when the indicated platform speed is known but the cross-current in the water path is unknown. A sinusoidal frequency equalling the PRF would give the same error from pulse to pulse and lead to a displaced, but sharply imaged target. A very high sinusoidal frequency trends towards the alias of a lower-frequency platform motion.

\subsection{Signal Processing Effects}

This section shows the results of four forms of signal processing effects on imagery. The first two, cross-track weighting and along-track weighting, are well known signal processing effects associated with windowing and weighting for real apertures. Essentially, these metheds allow a tradeoff to be made between narrow resolution in range and azimuth against the range and azimuth sidelobe level.

The final two signal processing effects are respectively for sampled operation (using narrow, broadband, and sub-apenures), and for undersampled operation using broadband signals to overcome azimuthal aliases.

The Gough and Hayes [1989] experiment again provides the framework for these examples.

\subsubsection{Cross-Track (Range) Weighting/Tapering}

Three range weights/tapers are available to reduce range sidelobes: the rectangular (or uniform), the triangle, and the half-cycle cosine. Note that all the images shown in this thesis thus far have used rectangular weighting. An example of each taper is shown in Figure 5.26

\subsubsection{Along-Track (Azimuth) Weighting/Tapering}

Two azimuthal weights/tapers are available to reduce azimuthal sidelobes: the rectangular (which has been used in all the original Figures shown in this thesis), and the Hanning. An example of each is shown in Figure 5.27. Like the range taper examples, the choice of an azimuthal taper reduces the sidelobe level at
the cost of a wider mainlobe. 
Figure 5.26 - Range Taper
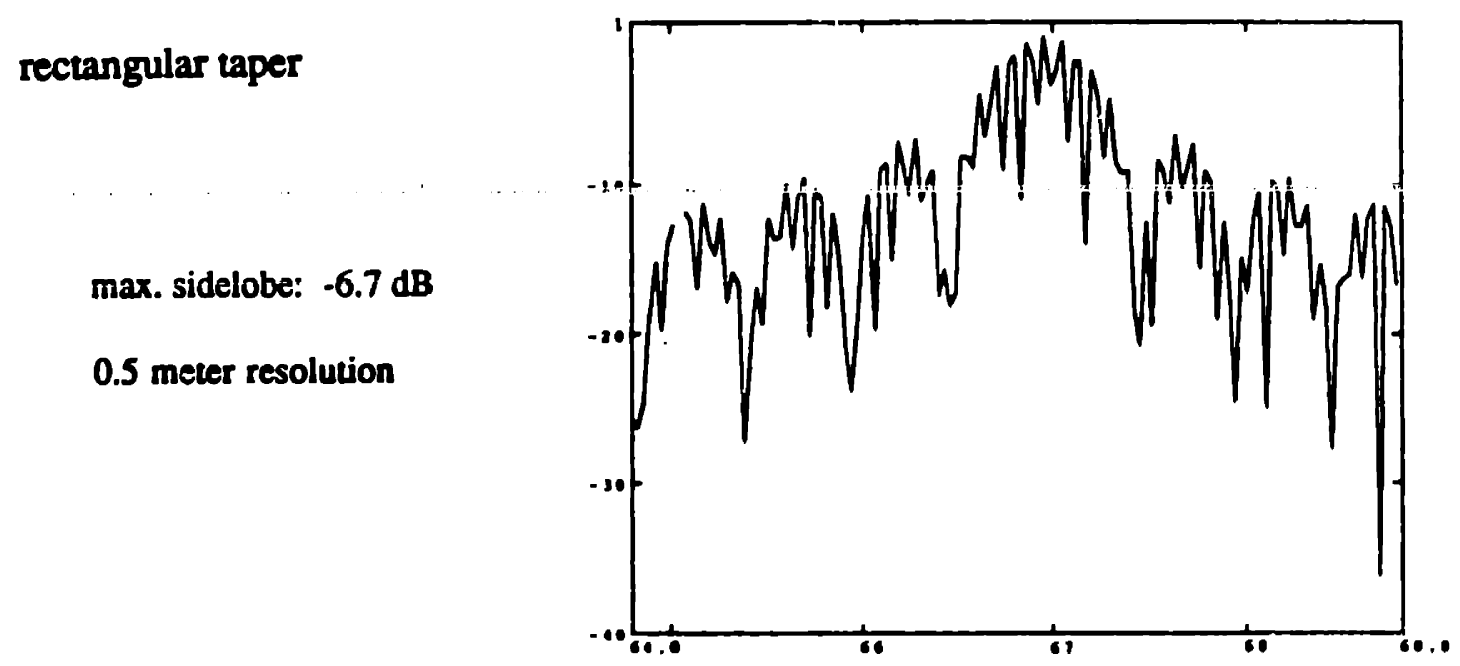

\section{triangle taper}

max. sidelobe: $-13.1 \mathrm{~dB}$

0.86 meter resolution

half-cycle cosine taper

max. sidelobe: $-11.5 \mathrm{~dB}$ 0.80 meter resolution

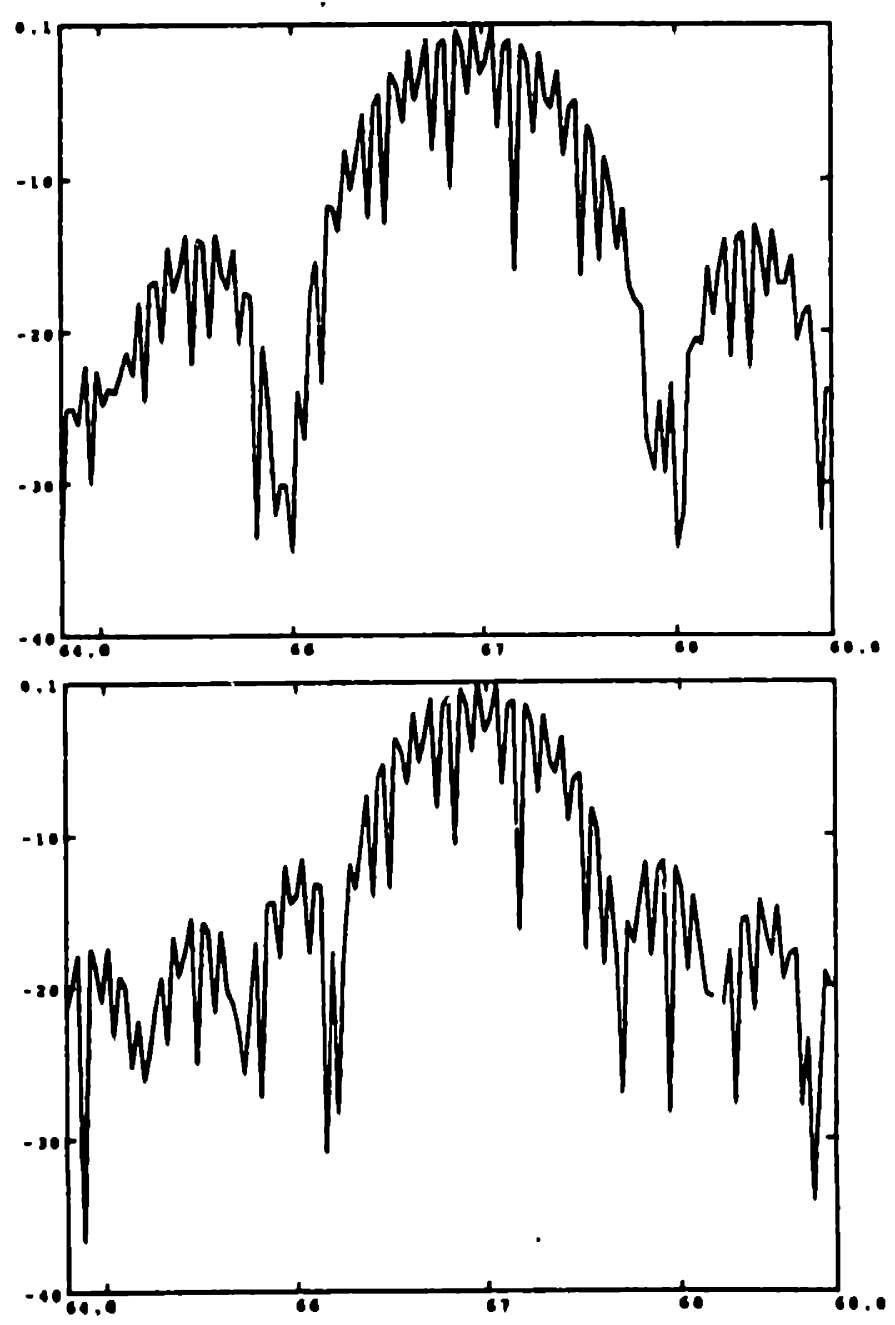


Figure 5.27 - Azimuth Taper

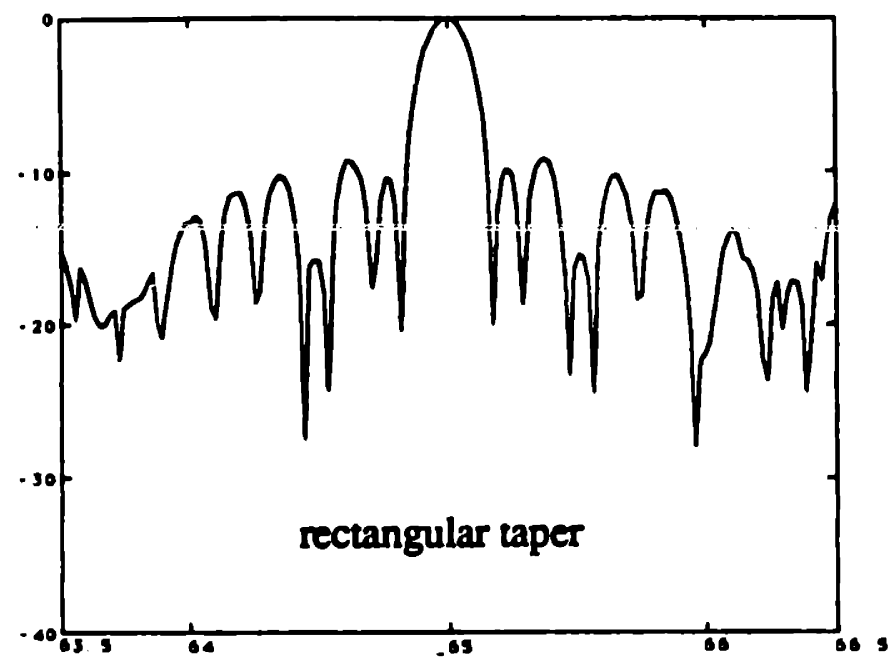

azimuth resolution: $20.5 \mathrm{~cm}$

max. sidelobe:

$-9.3 \mathrm{~dB}$ (mainlobe $=0 \mathrm{~dB}$ )

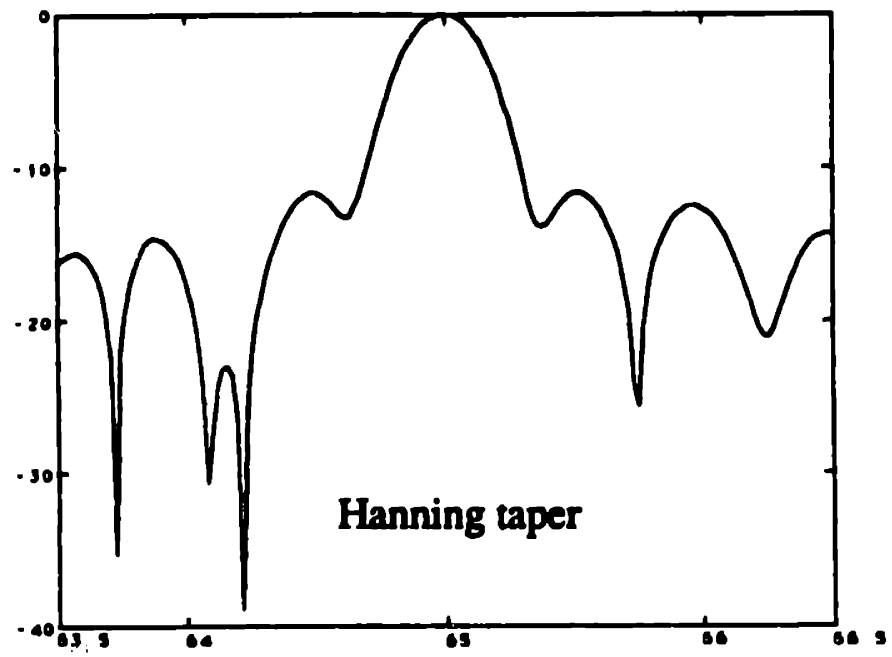

azimuth resolution: $34 \mathrm{~cm}$

max. sidelobe:

$-11.7 \mathrm{~dB}$ (mainlobe $=0 \mathrm{~dB}$ ) 


\subsubsection{Alias Levels at Nyquist Sampling Operation}

The results in this section refer to section 2.3.2 on the limitations to "Crushing" the alias lobe with the sonar beampattem null. The cases shown in this section were all modeled for a focused synthetic aperture sonar that moves exactly D/2 between pulses (which is the "sampling" or Nyquist criterion for a synthetic aperture radar or sonar).

For the cases shown, the mainlobe (i.e. the target lobe) always appears at $y=65.0$ meters: the alias closest to the mainlobe should appear at approximately $y=53.0$ meters for $f_{c}=28 \mathrm{kHz}$, and approximately at $y=49.8$ meters for $f_{c}=22.5 \mathrm{kHz}$. The alias levels shown in Table 5.6 are the largest levels observed in the neighborhood of the alias location for $f_{c}$ (note that the alias doesn't exist at a unique $y$-location for broadband operation - the alias lobes move as the transmit frequency sweeps across the band). $\mathrm{N}$ is the number of sub-aperture lengths the total aperture is divided into, and the alias level is the number of $\mathrm{dB}$ (magnitude) for the alias relative to zero $\mathrm{dB}$ for the target mainlobe. Only one sub-aperture length is used to form each image, and the ratio $1 / N$ is the portion of the aperture used, so these data correspond to a single look, at an N-partition aperture, from the broadside position sub-apenture.

Four cases case are listed in Table $\mathbf{5 . 6}$ for azimuthal image slices taken at the target slant range for five values of $\mathbf{N}$, the first three cases focused and the last unfocused. The second and third cases correspond to the Gough and Hayes experiment, even though they did not move their platform slowly enough to satisfy spatial sampling (hereafter referred $t 0$ as Nyquist-rate sampled). The first case is intended to show a very narrowband comparison to the other two. The remarks 'ridge' and 'bump' in Table 5.6 refer to general shape of the largest alias in the image, 'smeared' refers $\omega$ an alias which is indistinguishable from the nonalias sidelobes, and 'nulled' means a significant null is located where the alias should occur. Example images for $\mathbf{N}=1$ and $\mathbf{N}=\mathbf{8}$ for the first three cases in the table are shown respectively in Figures 5.28, 5.29 and 5.30.

Five general observations may be made from these results. The first two observations concern the 28 $\mathrm{kHz}$ cases. First, the reduction in the level of the alias target image is shown for both the $10 \mathrm{~Hz}$ bandwidth and $750 \mathrm{~Hz}$ bandwidth cases when the full $-3 \mathrm{~dB}$ to $-3 \mathrm{~dB}$ aperture length is subdivided by $\mathrm{N}$. The second observation is that the $750 \mathrm{~Hz}$ bandwidth does a better job at reducing the level of the alias image as compared to a corresponding $\mathrm{N}$-value for the $10 \mathrm{~Hz}$ bandwidth. 
Table 5.6 - Alias Level Relative to Mainlobe $\mathbf{N}$ alias level

remarks, y-position

\begin{tabular}{|c|c|c|c|}
\hline$f_{c}=28 \mathrm{kHz}$ & 1 & -11 & bump (53.1) \\
\hline \multirow[t]{4}{*}{$B W=10 \mathrm{~Hz}$} & 2 & -17 & bump (53.1) \\
\hline & 4 & -23 & smeared (53.1) \\
\hline & 8 & -30 & nulled (53.0) \\
\hline & 16 & -34 & nulled (53.0) \\
\hline$f_{c}=28 \mathrm{kHz}$ & 1 & -13 & bump (53.6) \\
\hline \multirow[t]{4}{*}{$\mathrm{BW}=750 \mathrm{~Hz}$} & 2 & -24 & bump (53.6) \\
\hline & 4 & -36 & smeared (53.0) \\
\hline & 8 & -38 & nulled (53.0) \\
\hline & 16 & -39 & nulled (53.0) \\
\hline$f_{c}=22.5 \mathrm{kHz}$ & 1 & -14 & ridge $(57.5)$ \\
\hline \multirow[t]{4}{*}{$\mathrm{BW}=15 \mathrm{kHz}$} & 2 & -13 & bump (57.9) \\
\hline & 4 & -21 & bump (57.5) \\
\hline & 8 & -21 & bump (57.5) \\
\hline & 16 & -22 & smeared (57.5) \\
\hline$f_{c}=28 \mathrm{kHz}$ & 1 & less than - 31 & nulled $(53.0)$ \\
\hline $\mathrm{BW}=750 \mathrm{~Hz}$, & & & \\
\hline
\end{tabular}

Notes: $V_{0}=.1875$ and $P R F=1.25 \mathrm{~Hz}$ (@ Nyquist ratc),

$R_{0}=66.8$ meters to point target; $t=.040$ seconds,

$D=0.30$ meters, $B W=f_{2}-f_{1}$, focused unless otherwise stated.

The third observation is that the $15 \mathrm{kHz}$ bandwidth, $\mathrm{f}_{\mathrm{c}}=22.5 \mathrm{kHz}, \mathrm{N}=1$ case has no improvement over the $750 \mathrm{~Hz}$ bandwidth, $28 \mathrm{kHz}, \mathrm{N}=1$ case. This suggests that for a Nyquist sampled synthetic aperture sonar, increases in bandwids must be used with caution because they won't necessarily give better alias image rejection. This is especially dramatic for $N \geq 2$, where the narrower bandwidth case has an alias image rejoction that is at least $10 \mathrm{~dB}$ better than the wideband case. 


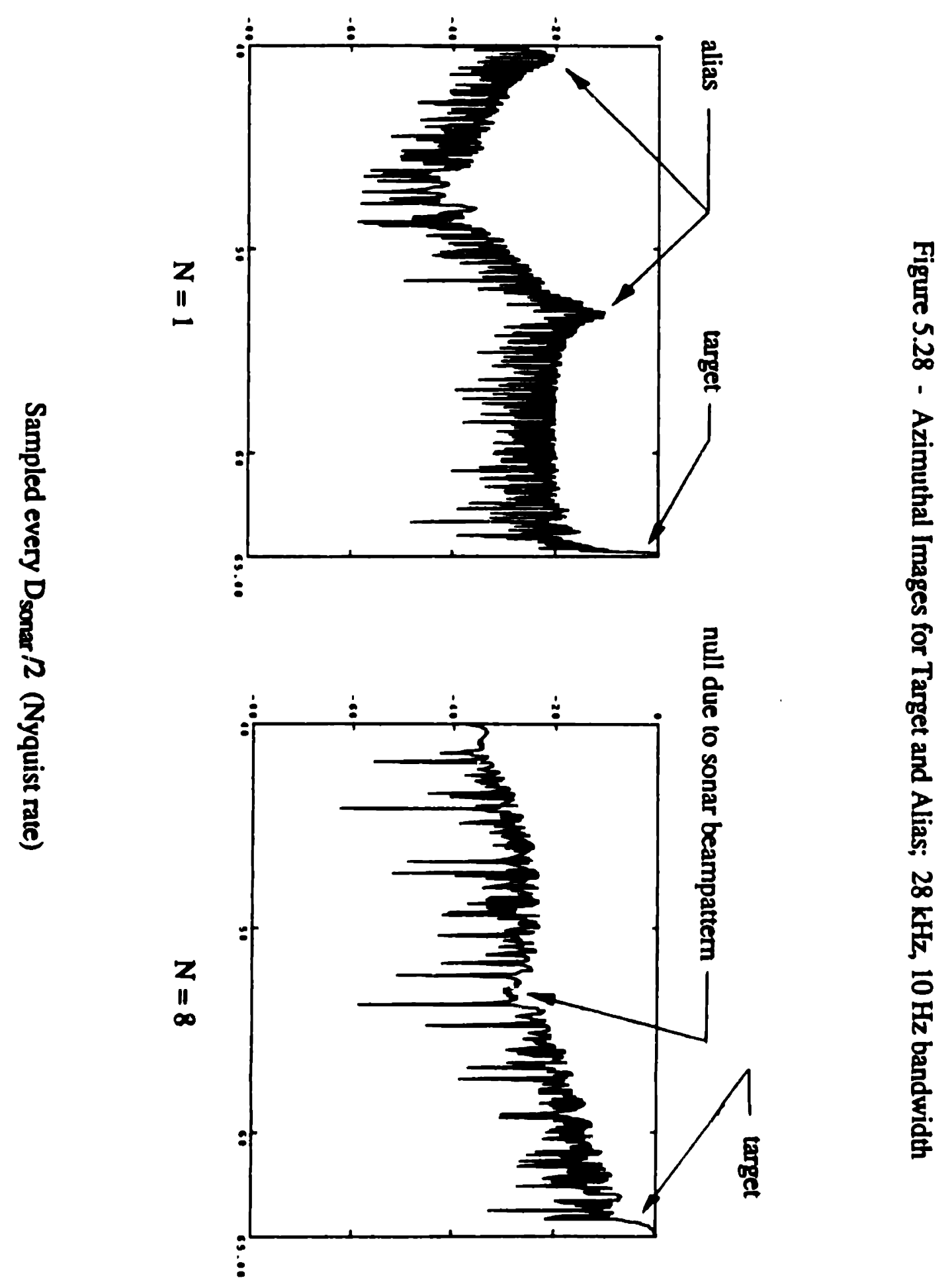


홍
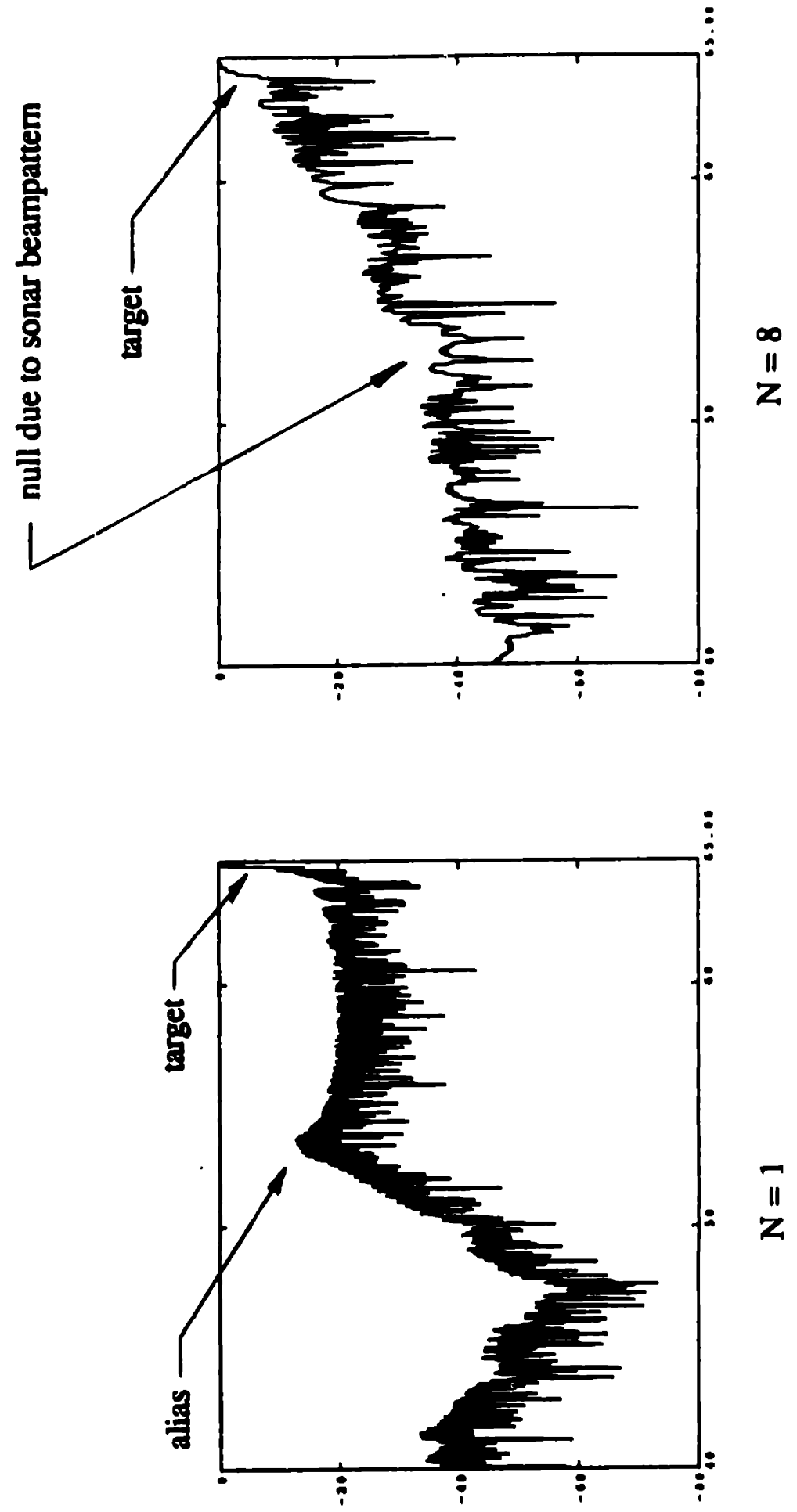

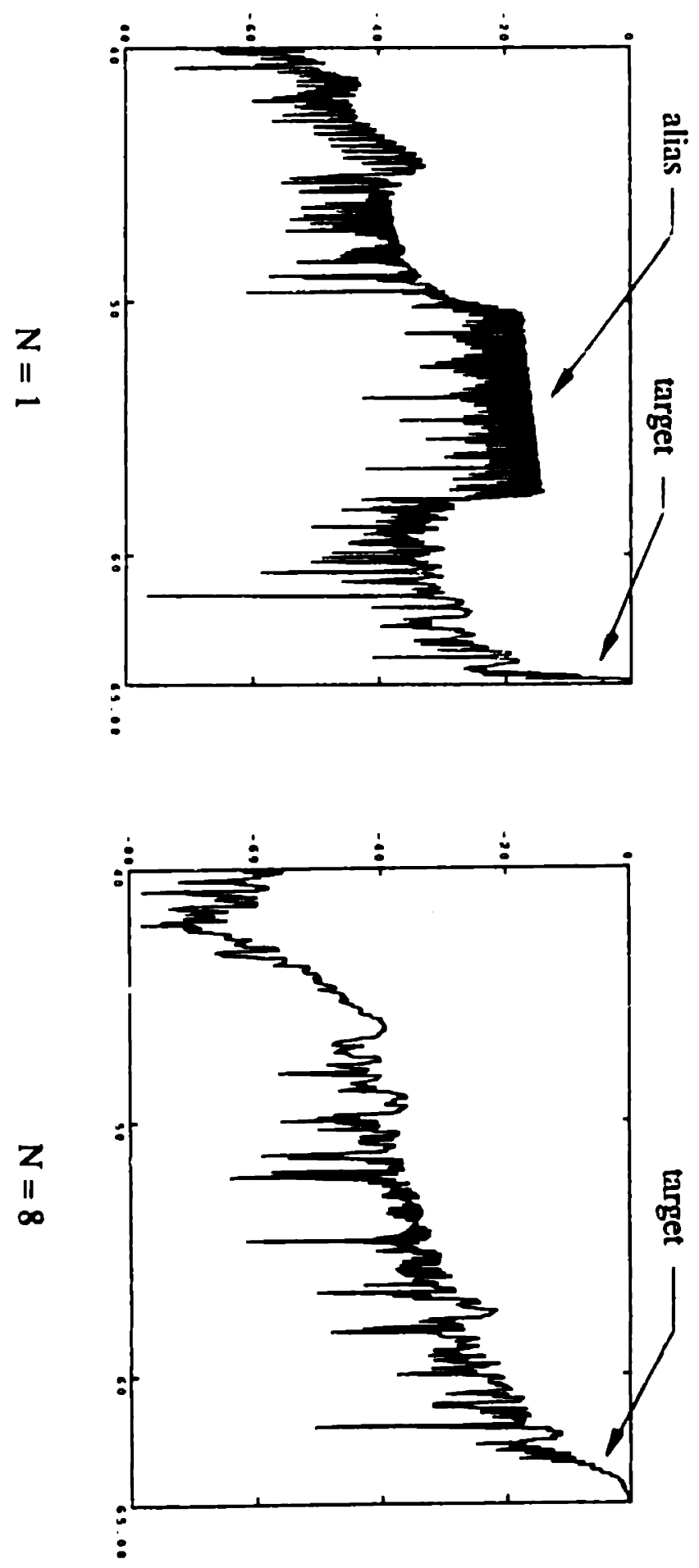

苞

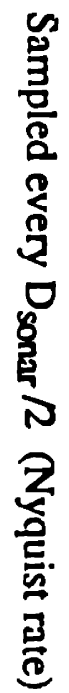

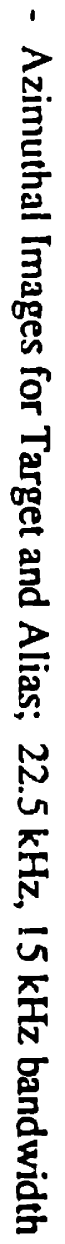


Figure 5.31 - Unfocused Azimuthal Image Showing Alias Rejection

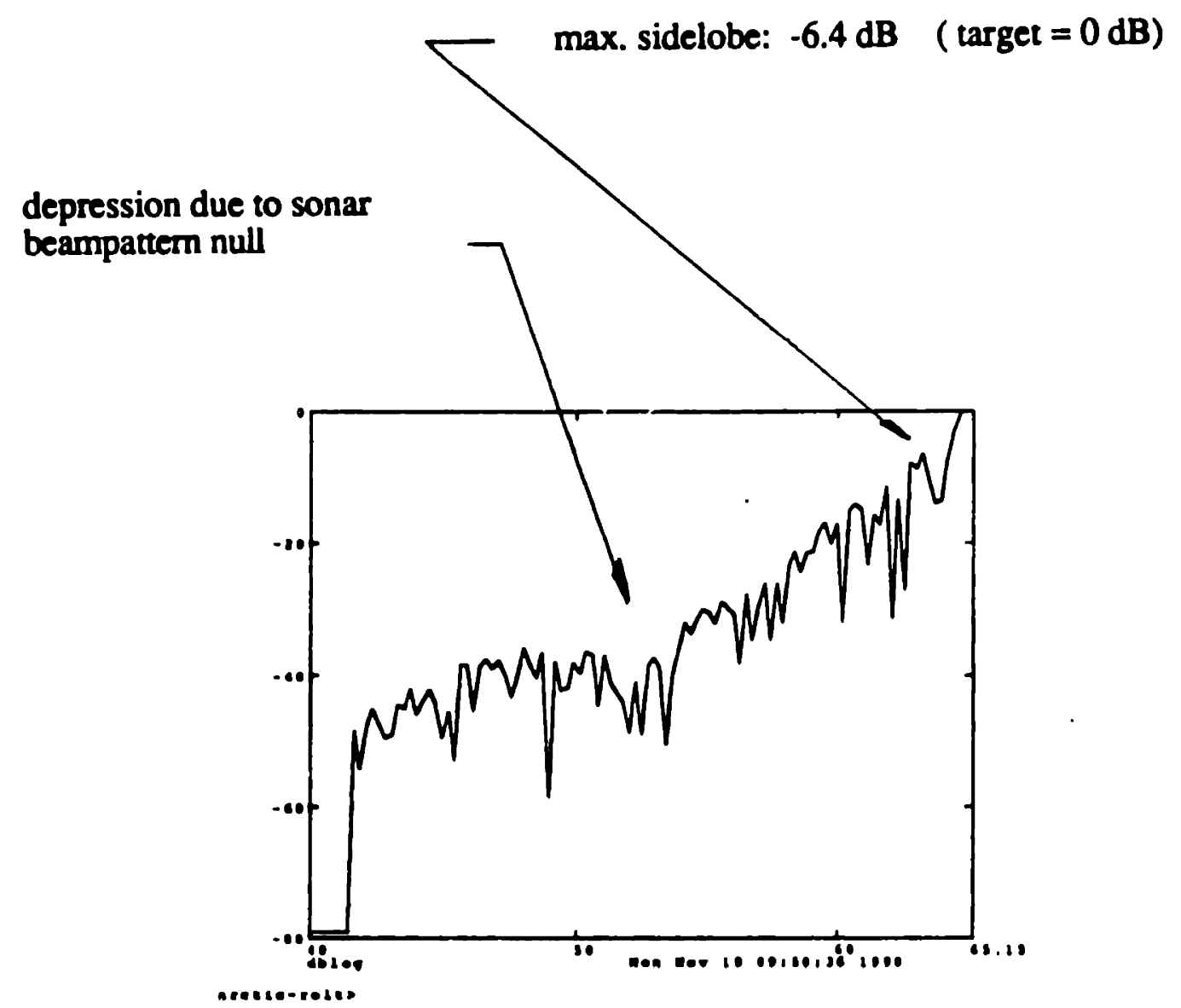

resolution: $.94 \mathrm{~m}$ (agrees $\mathrm{w} /$ theory) 
One final note concerning the use of sub-apertures for Nyquist-sampled focused synthetic apertures is that the sub-aperture length, as it is made progressively smaller, approaches that for a unfocused synthetic aperture. Because the use of sub-apertures in image processing reduces the alias level, then a Nyquistsampled unfocused aperture should likewise have good rejection of aliases. This is verified by a single example in Figure 5.31, and is the last entry in Table 5.6.

The subdivision of a Nyquist-rate sampled, full synthetic aperture to reduce alias images bears more scrutiny, particularly for SARs. Table 5.7 shows a comparison of bandwidth to center frequency ratios for SAR and SAS. The SAR examples (from both airborne and spaceborne platforms) show the lowest and highest values for $B W / f_{c}$ among the (unclassified) SARs listed in Synthetic Aperture Radar, Technology and Applications [1989], and range from .0016 to .08. Of the 13 airbome and spaceborne SARs listed (see pp. 13-141, 13-143, 14-113, 14-137) all but one were below $B W / f_{c}=.015$. This suggests that SARs are predominanuly narrowband devices if we assume broadband is $B W>.1 f_{c}$. This sharply contrasts with synthetic aperture sonars. An equivalent survey of SAS in the literature was not possible (the authors seldom published the transmit bandwidth), but of those that did, the $B W$ to $f_{c}$ ratio is considerably higher.

Since SARs may be classified as narrowband, then th:y must overcome strong aliases by means of dividing a full aperture into $\mathrm{N}$ sub-apertures, because there is litule bandwidth to assist in smearing the alias target lobes. The alias target image levels shown in Table 5.6 (especially the narrowband $28 \mathrm{kHz}, \mathrm{BW}=10$ $\mathrm{Hz}$ case) are consistent with those cited by R.L. Jordan [1980] for the Seasat-A SAR, where "the level of integrated ambiguities in azimuth is estimated to be between - 18 and $-24 \mathrm{~dB}$..." Recall that Seasat was a 4 look spaceborne SAR (see Table 2.2). This is a reasonable comparison between SAS (simulated) data and real SAR data because the alias target levels (or ambiguity-to-signal ratio, ASR) are not dependent on the propagation medium (i.e. radar vs. sonar); but are only dependent on the relations between wavelength $\lambda$, the transceiver length $D$, the speed $V_{0}$, and the PRF, and $N$ (the ratio of the full, available, aperture to the length of the processed aperture). 
Table 5.7 - BW to $f_{c}$ Ratios for SAR \& SAS

$$
\text { lowest } \mathrm{BW} / \mathrm{f}_{\mathrm{c}} \quad \text { highest } \mathrm{BW} / \mathrm{f}_{\mathrm{c}}
$$

\begin{tabular}{|c|c|c|}
\hline airbome SAR & .0016 & .08 \\
\hline & Aero Service GEMS & ERIM P-3 at L-band \\
\hline spacebome SAR & .0047 SIR-B & .0148 Seasat \\
\hline & .004 Eos SAR (1995) & .016 Eos SAR (1995) \\
\hline & & .016 SIR-C (1991-1992) \\
\hline SAS & .026 to .666 (variable). & - $23 \mathrm{kHz}$, Gough and Hayes [1989] \\
\hline & $-.222, \mathrm{f}_{\mathrm{c}}=4.5 \mathrm{kHz}$, Dutk & ewicz and Denbigh reservoir/sea lests [1987] \\
\hline & $?, \mathrm{f}_{\mathrm{c}}=20-100 \mathrm{kHz}$, & ICSC [1982]; ? NCSC [1974] \\
\hline
\end{tabular}

note: SAR data from Synthetic Aperture Radar, Technology and Applications [1989]. SAS data as cited. 


\subsubsection{Broadband Undersampled Operation}

The concept of a broadband undersampled synthetic aperture (or array) is essentially unknown in the radar literature primarily because of the high propagation speed of a radar wave; SARs for airbome and spacebome applications are able to sample echo data at least once for every $D / 2$ translation by the radar platform. This isn't generally true for the synthetic aperture sonar. The comparatively slow acoustic wave demands a combination of a slow platform and a limited range. Though there are operational circumstances where the sampled synthetic aperture sonars may be used, it would be certainly advantageous to allow higher platform speeds which therefore offer higher mapping rates. Higher platform speeds and/or greater imaging ranges combine to give a higher mapping rate, and this is always a desirable feature in underwater exploration. Hence, an increased mapping rate is the primary motivation for studying the broadband undersampled synthetic aperture.

In Chapter 2.4.2, the notion of the broadband undersampled synthetic array was described, and a "ballpark" algebraic expression was developed to estimate the level of alias smear (in $\mathrm{dB}$ ) relative to the mainlobe. This has also been described as the ambiguiry-10-signal ratio (ASR) in radar.

The section illustrates examples of broadband undersampled operation, using two sizes of subapertures $(N=1$ and $N=4)$. The test case will be the Gough and Hayes example from section 5.1.1 with the parameters listed in Table 5.1.

The $\mathrm{N}=1$ case (i.e. the full $-3 \mathrm{~dB}$ to $-3 \mathrm{~dB}$ aperture width) is summarized in Table 5.8, where a comparison is made between the ballpark equation 2.74 and the results from the computer model. An ensemble of broadband images for our framework wideband Gough and Hayes [1989] experiment is shown in Figure 5.32 for the $.517, .35$ and $.25 \mathrm{~m} / \mathrm{s}$ speeds. Note that the Gough and Hayes experiment used only a single speed $(.517 \mathrm{~m} / \mathrm{s}$ ). The point target for these images is at azimuth $y=65.0$ (and slant range $=66.8$ $m$ ). The convention from the previous section of showing only the azimuthal image slice and omituing the range image slice will be continued here.

The smeared alias target appears as an azimuthal (along track) ridge. Accompanying this ridge are uncluttered, symmetrical azimuthal regions straddling the target that are void of strong scauterers. These zones are then followed by the azimuthal alias ridge, which is stronger near the target, and weaker away from the target. The slope of the ridge is a result of the natural beampattem of the sonar transducer, which gives alias images close to the target more amplitude weight than alias images further away from the targët. 
Figure 5.32 - Broadband Undersampled Azimuth Images of Target and Smeared Aliases, Full Aperture $(\mathrm{N}=1)$

$$
\begin{aligned}
& \mathrm{f}=22.5 \mathrm{kHz} \\
& \mathrm{BW}=15 \mathrm{kHz} \\
& \text { full aperture }(\mathrm{N}=1)
\end{aligned}
$$

$.517 \mathrm{~m} / \mathrm{s}$

$.35 \mathrm{~m} / \mathrm{s}$

$.25 \mathrm{~m} / \mathrm{s}$
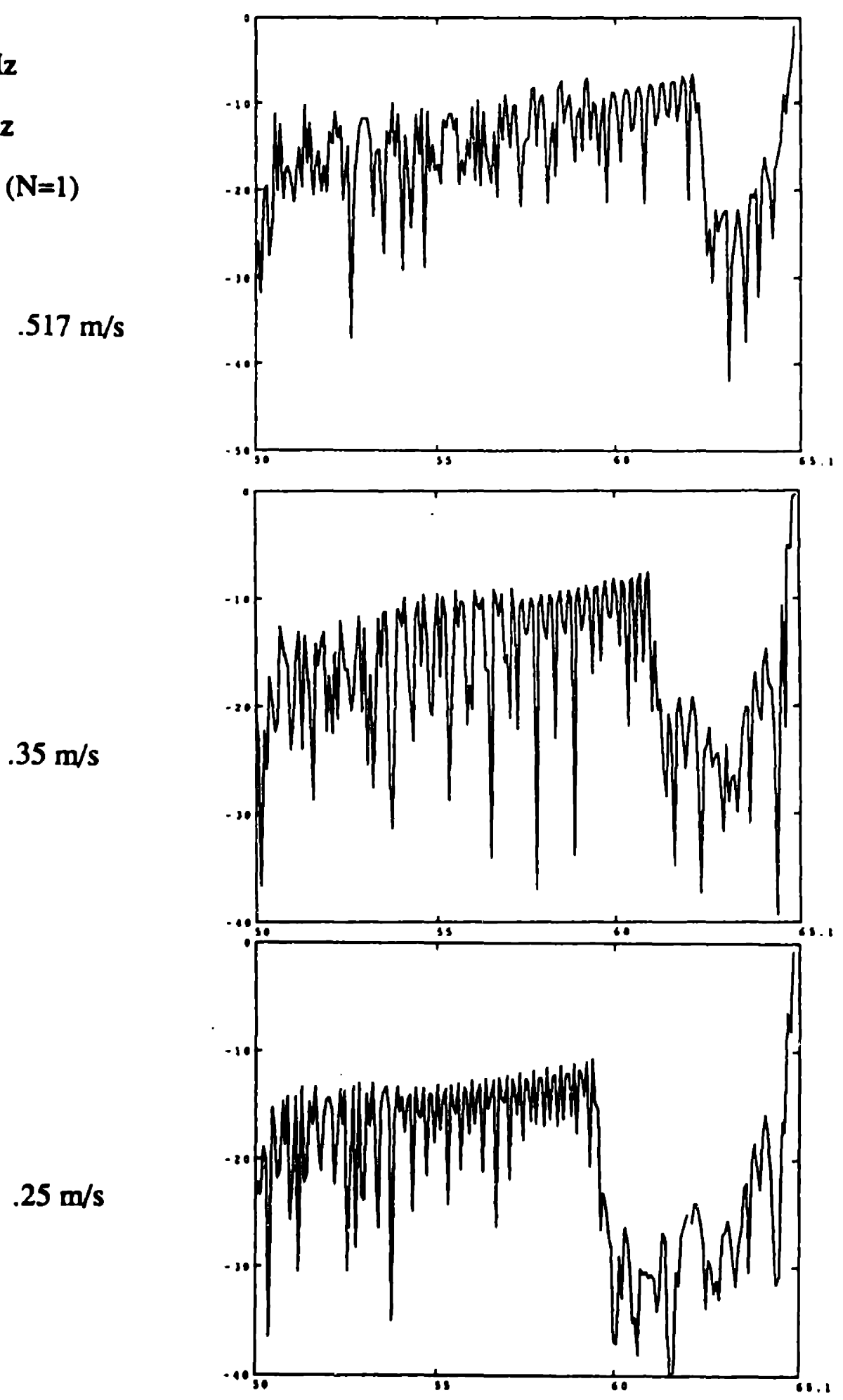
The comparison between equation 2.74 and the results from the computer model are shown for two test cases in Table 5.8. The computer model results lists both the level for the highest-level peak in the smeared alias ( "maximum"), and the average level for the smeared alias ("average").

Table 5.8 Broadband Undersampled Alias Levels Compared:

Equation 2.74 vs. Computer Model, $N=1$

\begin{tabular}{|c|c|c|c|c|}
\hline \multirow[t]{2}{*}{ Case } & \multirow{2}{*}{$\begin{array}{c}V_{0} \\
(\mathrm{~m} / \mathrm{s})\end{array}$} & \multirow{2}{*}{$\begin{array}{l}\text { equation } 2.74 \\
\text { (dB) }\end{array}$} & \multicolumn{2}{|c|}{ computer model (dB) } \\
\hline & & & maximum & average \\
\hline \multirow[t]{5}{*}{1} & .517 & -0.9 & $=0$ & 0 \\
\hline & .40 & -1.5 & $\approx 0$ & 0 \\
\hline & .35 & -1.8 & $\approx 0$ & 0 \\
\hline & .30 & -2.1 & -1.6 & -1.6 \\
\hline & .25 & -2.5 & -5.1 & -5.1 \\
\hline \multirow[t]{5}{*}{2} & .517 & -8.6 & -6.4 & -7.7 \\
\hline & .40 & -9.2 & -7.0 & -8.2 \\
\hline & .35 & -9.5 & -7.6 & -8.7 \\
\hline & .30 & -9.8 & -8.7 & -10.2 \\
\hline & .25 & -10.2 & -10.7 & -12.3 \\
\hline
\end{tabular}

notes: Case 1. $f_{c}=28 \mathrm{kHz}, \mathrm{BW}=750 \mathrm{~Hz}, P R F=1.25 \mathrm{~Hz}, \mathrm{D}=.30 \mathrm{~m}, \mathrm{~N}=1, \mathrm{R}_{\mathrm{o}}=66.8 \mathrm{~m}$. Case 2. $\mathrm{f}_{\mathrm{c}}=22.5 \mathrm{kHz}, \mathrm{BW}=15 \mathrm{kHz}, \mathrm{PRF}=1.25 \mathrm{~Hz}, \mathrm{D}=.30 \mathrm{~m}, \mathrm{~N}=1, \mathrm{R}_{\mathrm{o}}=66.8 \mathrm{~m}$. $V_{0}<.1875 \mathrm{~m} / \mathrm{s}$ is the speed needed to satisfy the sampling requirement.

Several comments must now be made concerning the results shown in Table 5.8. First, the narrowband example in the table (Case 1 ) is shown for comparison to the broadband results (Case 2). It does not compare well with equation 2.74, and indeed it shouldn't. Second, the $V_{0}=.517 \mathrm{~m} / \mathrm{s}$ Gough and Hayes [1989] example listed as part of Case 2, has reasonable agreement between the computer model and equation 2.74 , over the speed range .25 to $.517 \mathrm{~m} / \mathrm{s}$. The $-7.7 \mathrm{~dB}$ (avg.) value for the $.517 \mathrm{~m} / \mathrm{s}$ speed also substantially agrees with the experimental results of Gough and Hayes [1989] and in Hayes [1989]; I have estimated the maximum smeared alias at $-8.2 \mathrm{~dB}$ in two of their experimental images (see page 130, Fig. 7.5 in Hayes [1989], as reprinted in Fig. 5.5 in this thesis; see also Fig. 6, p. 2332 in Gough and Hayes [1989]; another image in Fig. 7.9 in Hayes [1989].) 
Hayes however, in the computer simulations in his thesis (see Chap. 6, pp. 114-124, in Hayes [1989]), shows that for coherent processing of the conditions listed for Case 2, speed $.517 \mathrm{~m} / \mathrm{s}$, the "minimum signal to self-clutter ratio is $24 \mathrm{~dB}$ " (the signal to self-clutter ratio is the level of alias smear described in this section). The $-24 \mathrm{~dB}$ value Hayes estimated appears to disagree with his own experimental measurements and with those shown here.

Since sub-aperture processing was a benefit to the Nyquist-rate sampled synthetic aperture, a subaperture approach was likewise altempted here for the broadband undersampled synthetic aperture sonar. Only three examples of an $\mathbf{N}=4$ focused process for a broadband undersampled synthetic aperture are shown, along with a comparison between the model and equation 2.74. The results are listed in Table 5.9, and the images are shown in Figure 5.33.

These images clearly demonstrate that sub-apenures are not useful for a broadband undersampled synthetic array, and the results of Table 5.9 demonstrate the ill effects. Essentially, by using sub-apertures in the processing, fewer pulses are coherenty combined to form a target image. Since the broadband undersampled syathetic aperture achieves performance by capitalizing on relatively poor range correlations at alias target locations, especially at the ends of the synthetic aperture, then the use of sub-apertures trims an important part of the processing, rendering the technique useless because the aliases grow in level instead of diminishing. The results of Table 5.9 show that the broadband undersampled synthetic aperture should always include the full aperiure, or $\mathbf{N}=1$.

Table 5.9 Broadband Undersampled Alias Levels Compared:

Equation 2.74 vs. Computer Model, $N=4$

$V_{0} \quad$ equation $2.74 \quad$ computer model (dB)

$(\mathrm{m} / \mathrm{s})$

maximum average

\begin{tabular}{llll}
\hline .517 & -10.1 & -6.2 & -6.9 \\
.40 & -10.7 & -4.7 & -6.2 \\
.35 & -11.0 & -5.2 & N/A
\end{tabular}

notes: $f_{c}=22.5 \mathrm{kHz}, \mathrm{BW}=15 \mathrm{kHz}, \mathrm{PRF}=1.25 \mathrm{~Hz}, \mathrm{D}=.30 \mathrm{~m}, \mathrm{~N}=4, \mathrm{R}_{\mathrm{o}}=66.8 \mathrm{~m}$.

$V_{0}<.1875 \mathrm{~m} / \mathrm{s}$ is the speed needed to satisfy the sampling requircillint.

N/A - not available 
Figure 5.33 - Broadband Undersampled Azimuth Images of Target and Smeared Aliases, Reduced Aperture $(N=4)$

$\mathrm{f}=22.5 \mathrm{kHz}$
$\mathrm{BW}=15 \mathrm{kHz}$
reduced aperture $(\mathrm{N}=4)$

$.517 \mathrm{~m} / \mathrm{s}$

$.40 \mathrm{~m} / \mathrm{s}$
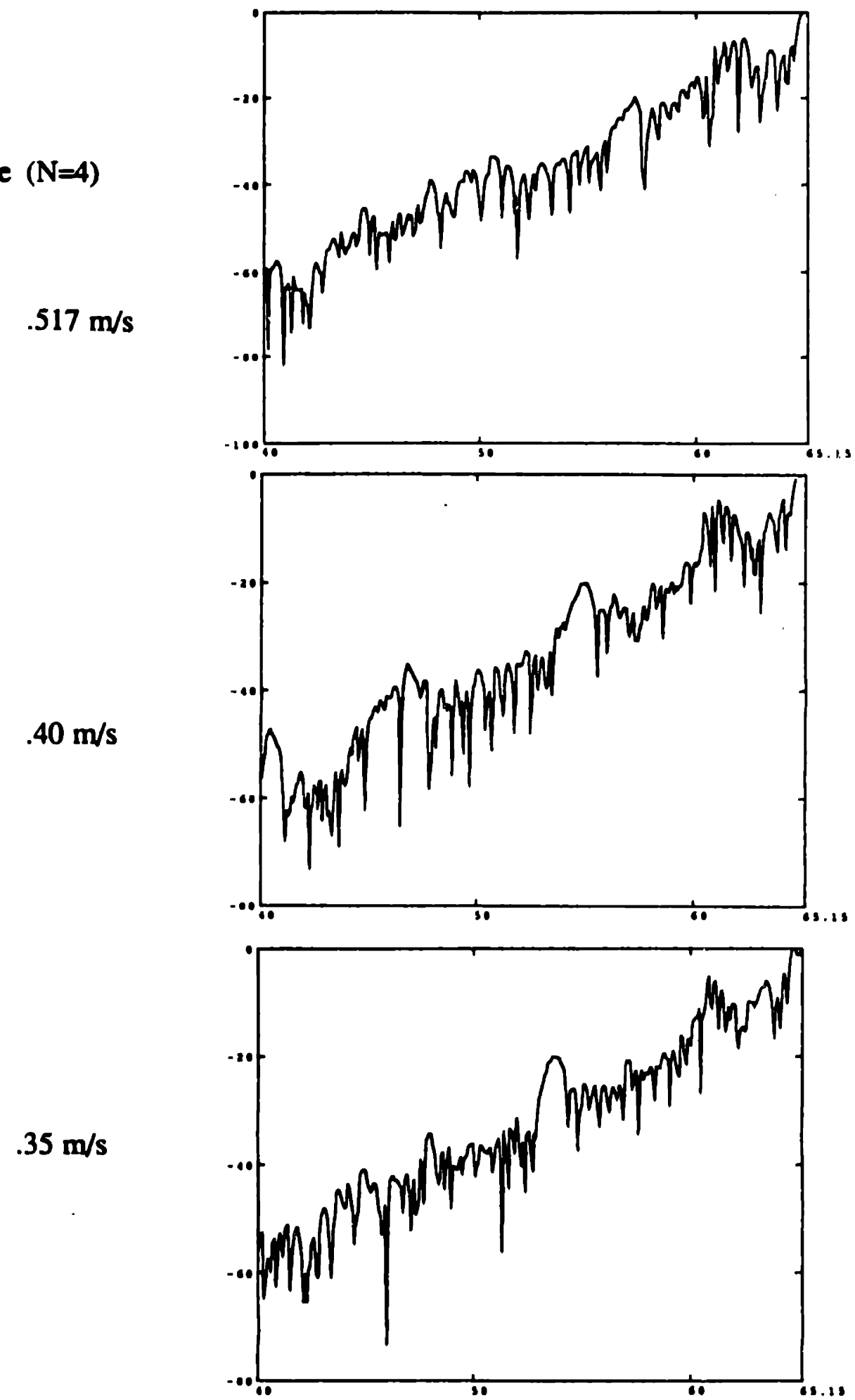
By this same line of reasoning, in contrast, it may be more advantageous to use a wider aperture in the processing (i.e. the full null-to-null beamwidth, instead of the usual $-3 \mathrm{~dB}$ to $-3 \mathrm{~dB}$ beamwidth) because more data is available to match to the true target during the correlation processing, and likewise more data is available to reduce the level of the alias smear. A single example is sufficient to show this, again using the Gough and Hayes, $15 \mathrm{kHz}$ bandwidth, $1 \mathrm{knot}(.517 \mathrm{~m} / \mathrm{s})$ example as the framework. An azimuthal slice image is shown in Figure 5.34 for a single point target (at a slant range of $66.84 \mathrm{~m}$.), where the target is at the azimuth location 65.0 meters. The processing used the nearly the full mainlobe null-to-null beamwidth (approximated at the center frequency of $22.5 \mathrm{kHz}$ ) which contrasts the $-3 \mathrm{~dB}$ to $-3 \mathrm{~dB}$ beamwidth used elsewhere throughout this thesis. The maximum smear lobe was - $11.5 \mathrm{~dB}$ (relative to the target), which favorably compares to the $-6.4 \mathrm{~dB}$ level found for the same sonar using the $-3 \mathrm{~dB}$ to $-3 \mathrm{~dB}$ mainlobe (see Table 5.8). The azimuth resolution was $15 \mathrm{~cm}$ (agrees with theory), and there are about 8 alias peaks from -11.5 to $-13.5 \mathrm{~dB}$ in the neighborhood of 57 to 63 meters (azimuth). This example shows that the broadband undersampled SAS not only demands that the full apenure be processed, but also that the performance in alias rejection improves by about $6 \mathrm{~dB}$ when the null-to-null mainlobe beamwidth is used to determine the aperture length. 
Figure 5.34 - Broadband Undersampled Azimuth Image of Target and Smeared Aliases, Null-to-Null Aperture

$$
\begin{aligned}
& \mathrm{f}=22.5 \mathrm{kHz} \\
& \mathrm{BW}=15 \mathrm{kHz}
\end{aligned}
$$

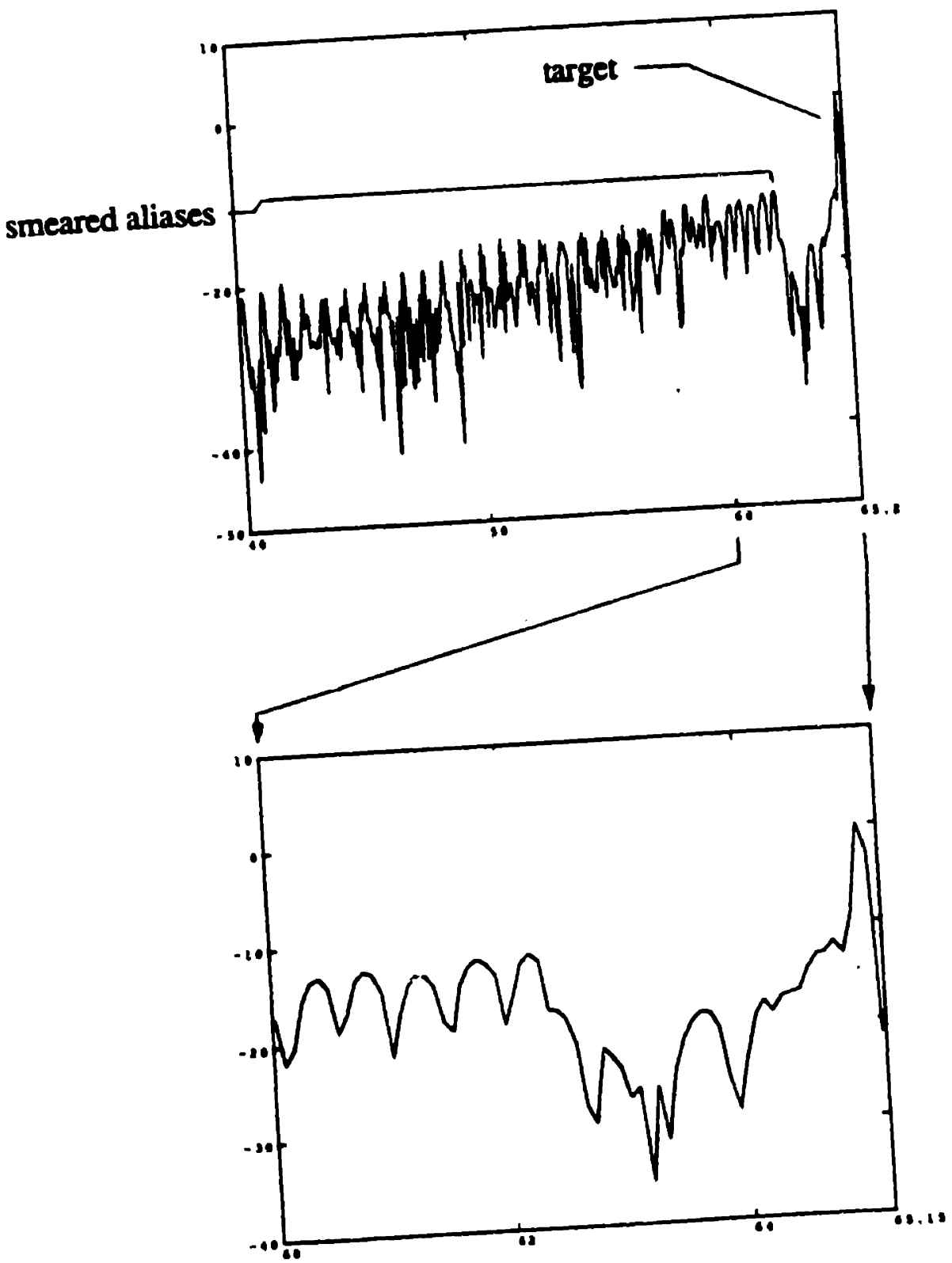




\section{Chapter 6}

\section{CONClusions AND Future WORK}

\subsection{Conclusions}

The first conclusion is that the SAS model developed has been verified by direct comparison with the Gough and Hayes [1989] experiment using an undersampled focused SAS. In the experiment, undersampling caused alias images to appear, in addition to the actual target, when the images were created using a narrowband transmit signal. These aliases were further shown to smear in the azimuthal direction when a broadband transmit signal was used. The computer model developed for this thesis showed the same results, and several image features were directly compared, and agreed, to those in the experiment:

- the target was correcty located, and the range and azimuthal resolutions agreed with the experiment,

- narrowband processing showed the existence of discrete alias target images,

- broadband processing showed the alias targets to be smeared along the azimuth,

- smeared alias targets agreed in both location and level (relative to the target, in dB),

- a mismatched image reconstruction platform speed showed azimuthal target image displacement.

The verification of the focused portion of the computer model allowed an exercise of the unfocused processing feature (Gough and Hayes did not perform any unfocused processing so there is no direct experimental comparison). The results agreed substantially with the theory for the unfocused synthetic aperture.

Several other conclusions concern the ocean effects on SAS. The first is that multipath due to surface and bottom influences are mitigated, largely due to the time-varying nature and predominant irregularity of the surface, and the absorption and occasional irregularity of the bottom. Since the SAS is a phase coherent system, the multipaths which involve surface and bottom reflections lose their phase coherency as compared to the direct path. Upon coherent imaging, these multipaths tend to be significantly reduced relative to the direct path target. The case for long-range multipath not necessarily involving the surface and bottom was not explored in this thesis because the short-range ocean issues and platform influences are more important and are a focus of this thesis. 
The effect of a 'deep' ocean path on SAS imagery substantially depends on the sound speed profile. In the absence of a good estimate for the profile, the beamwidth for the sonar platform must be fairly narrow so that the imaging process is insensitive to any mismatch between the real ocean and the 'guess' ocean used to reconstruct the image. A poor guess of the refraction profile will properly image the target but at an incorrect range position. On the other hand, if the ocean sound speed profile is reasonably well known, a wide beam sonar may be used which will lead to both high resolution target imagery and correct range image location. The model used in this thesis then becomes very important in identifying the widest beam sonar capable for deep ocean imaging when the sound speed profile is uncertain.

The coherence time in the medium was shown to have an influence on SAS imagery, as expected. It was shown that the image effectively 'spreads' in range and in azimuth, but the image was still present even when the dwell time was twice the coherence time in the medium. This suggests that echo data from a target region should be processed using a number of dwell times, and the images compared. Features which show sharper resolution with increasing dwell time mean that the spatial-temporal coherence time limit was not exceeded. On the other hand, features showing degraded resolution and accompanying false targets (or apparently higher sidelobe levels) with increasing dwell time suggests that the coherence time limit was exceeded. By spanning a range of dwell times, the best possible image may be observed visually, and the coherence time may therefore be estimated.

A study of previous experiments concerning medium stability and coherence time (especially those concerning SAS) revealed dimensionless quantities for estimating coherence time based on the acoustic path length, the frequency, and the variance. These previous experiments, and the knowledge that images may even ba, formed for dwell times exceeding the coherence time (with some loss in image quality), further demonstrate that synthetic aperture sonar is possible and achievable in an ocean environment. SAS imagery is certainly sensitive to ocean spatial and temporal coherence, but it is surprisingly good at imaging in the presence of ocean variability.

The results of platform motion show similar effects to those of ocean variability. A SAS platform ideally travels on a perfectly straight path, or has sensors capable of measuring and correcting for spurious motion. However, the SAS platform has been shown to be capable of imaging a target even when subjected to a lateral displacement error envelope greater than the canonical $\lambda / 8$ (one-way; $\lambda / 4$ round-trip). A displacement error envelope of $\lambda / 2$ (one-way) gives an image having roughly half the amplitude, as compared to a platform having no motion error.

The signal processing effects encompassed azimuth and rarige weighting, spatially sampled operation, and spatially undersampled operation. The azimuth and range weighting of the synthetic aperture showed 
similar results to the weighting for a real aperture array. The natural amplitude weighting due to the sonar transducer beampattern was also shown to alter the sidelobe level depending on how much of the full aperture length was used during processing. The range was found to be -6.6 to $-20 \mathrm{~dB}$ (amplitude), with -10 $\mathrm{dB}$ being typical for synthetic apertures using the $-3 \mathrm{~dB}$ to $-3 \mathrm{~dB}$ sonar mainlobe.

Signal processing efferts on spatially sampled synthetic apertures (i.e. Nyquist-rate, or a transmission for at least every $\mathrm{D} / 2$ along-track displacement of the sonar) were primarily concerned with minimizing alias targets from images. It was also shown that the notion found in many SAS papers, and mostly overlooked in SAR papers, that the nulls of the transceiver beam pattern crush the alias image contributions, is false; the aliases exist and they occasionally show up even in SAR images. It was also demonstrated that processing portions of the full aperture, a sub-aperture approach, helps minimize the alias image contributions and reduces them to the level of the sidelobes. Thus, sampled syrthetic aperture sonars benefit from sub-aperture processing.

It was also suggested that the superposition of several sub-aperture images from the same full-aperture data accomplishes as much in reducing aliases as it does in reducing speckle thereby improving image quality (the improvement in image quality is usually attributed only to speckle reduction.)

The study of sampled synthetic apertures also showed that too much signal bandwidth does not necessarily improve alias image rejection.

The study of undersampled synthetic apertures (where a signal is not transmitted for every D/2 alongtrack displacement of the sonar) included a development of a "ballpark" equation for determining the level of smeared alias images relative to a target. The equation was shown to roughly agree with the computer model, provided that the aperture was undersampled and that the signal was broadband. Two other equations were developed to show the minimum bandvidth and transducer $Q$ (quality factor) necessary for useful operation. It was also shown that the broadband undersampled synthetic aperture benefits from the processing of the full-aperture (null-to-null) of the sonar transducer mainlobe, instead of using only the -3 $\mathrm{dB}$ to $-3 \mathrm{~dB}$ mainlobe (as is customary in the prior art of synthetic apertires, both radar and sonar), in order to reduce alias target images. This contrasts the sampled SAS which benefits from the sub-aperture approach. 


\subsection{Future Work}

The future work for synthetic aperture sonar may, in some sense, be guided by what has been suggested by this thesis, and by those results shown in Chapter 5. Many of the results shown here are new (at least as far as the unclassified literature is concerned) and they await experimental verification. The major thrust for future work is therefore experiment-based.

The synthetic aperture sonar essentially needs to be demonstrated in a working environment and not on a test rig, on rails, or on guides. The rail-based experiment of the U.S. Naval Coastal Systems Center (Loggins, C.D., J.T. Christoff, and E.L. Pipkin, [1982] ) and the wire-guided expsriment of Gough and Hayes [1989] have proven that ocean-based SAS can be made to work if the transverse platform motion is limited or non-existent; however only one experimental system (Dutkiewicz and Denbigh, 1987) has ever been tested without some form of guide, but the results were inconclusive because the target field was not verified by other means. A towed hydrodynamically stable SAS also needs to be tested without the use of motion compensation sensors; this experiment would then show whether synthetic aperture imaging is feasible without the added complication (and expense) of measuring the platform motion, and using the motion data in the image processing. This thesis suggests that this is feasible, but much of this depencis on the innate stability of the platform during transit, as well as on the wavelength of the signal. If so, (relatively) inexpensive synthetic aperture sonars may be designed and operated which have much better imaging performance than a conventional incoherent sidescan sonar.

On the other hand, an active synthetic aperture sonar needs to be designed and constructed having onboard sensors to measure platform motion, and the images should be reconstructed both with the sensor data, and without using the sensor data (i.e. no motion compensation). The sensors should be highly accurate and therefore promise to be expensive. This could be ideally be performed on a research submersible or submarine. Alternative ways to measure platform motion, for both surface and subsurface craft should be investigated, especially with an eye towards good performance and low cost. A SAS system would also be an excellent addition to a non-towed, untethered autonomous vehicle, where the sonar and plafform motion-sensing data are stored on-board for later reconstruction.

The tremendous quantity of work done in SAR should be heavily exploited for use in SAS, particularly in the areas of speed in image reconstruction (hybrid processing e.g.), and perhaps with an eye toward a real time system (this is being approached in contemporary SAR systems). 
Synthetic apertures in both sonar and radar are created on straight line paths, and they give rise to images with three dimensions: 2-D in $x-y$ geometry and 1-D in target strength (or target reflectivity). Experiments need to be performed such that the synthetic array formed is within a 2-D plane (but not in a straight line) as shown in Fig. 2.33, the 2-D "billboard" synthetic aperture. This type of an aperture for sonar or radar could, in principle, form four dimensional images: 3-D in geometry, and 1-D in target strength.

Beyond the general topic of synthetic aperture sonars which need to be built on an experimental/verification basis, the broadband SAS should also be experimentally investigated. Several forms of broadband signals are available including chirps (FM sweeps), and pseudorandom noise; the merits and disadvantages of these have not been studied other than as presented in this work, at least as far as synthetic aperture sonars are concerned. Another feature not discussed in this thesis, but which has been discussed in several papers in the bibliography (see Walsh, Raytheon reports e.g.), is the use of coded pulses. These pulses have different frequency content and can therefise be discriminated from each other so that they may be in transit in the water at the same time without fear of ambiguous echoes. This is one way to overcome the $D / 2$ sampling requirement for every pulse transmission. The coded pulses could be used in both the sampled and in the undersampled synthetic aperture sonars.

Finally, broadband signals for airborne and spaceborne SAR, either for sampled or undersampled operation, have not been studied in the unclassified literature (to the author's knowledge). The improvement in ambiguity rejection, and the capability to map faster could prove as useful in certain SAR applications, and it is in certain SAS applications. 


\section{Appendix A}

\section{DIRECTIVITY OF APERTURES}

This Appendix formulates the directivity of apertures, specifically rectangular apertures, which gives rise to the beam pattern.

Consider the rectangular piston sound radiator, the aperture, shown in Figure A.1. It resides in a rigid baffle of infinite extent in the yz-plane. The piston vibrates sinusoidally at (radian) frequency $\omega$ with uniform velocity so it is also, in a sense, rigid but nevertheless free to oscillate. The piston is deliberately made with a large aspect ratio $\mathrm{L} / \mathrm{W}$, because we are at first interested in the directivity of the piston in the xz-plane.

Figure A.1 also shows some other explicit and important geometry. The piston length $L$ (in the $z-$ axis direction) has an inscribed differential element $\mathrm{dz}$ which is vanishingly small compared to $\mathrm{L}$. The differential element $\mathrm{dz}$ is located at position $\mathrm{z}$ along the $\mathrm{z}$-axis, and the piston is centered at the origin of the $x-, y-$, and $z$-axes for convenience.

Observer point $O$ (often called a field point) is the point in the xz-plane on which we will base this analysis, because we seek the directional character of the piston as viewed from observer puint $O$, as $O$ marches about the origin at a constant radius $\mathbf{r}$ in the $\mathbf{x z}$-plane. Observer point $\mathbf{O}$ is at rectangular coordinates $\left(x_{0}, y_{0}, z_{0}\right)$ where $y_{0}=0$. Length $l$ is the distance from the center of the differential element $\mathrm{dz}$ to $O, \alpha$ is the angle between the $Z$-axis and $O$, and $\theta$ is the angle between the $X$-axis and $O$.

We start by allowing that the time-varying differential pressure dp at $\mathrm{O}$ is given by

$$
\frac{\mathrm{e}^{-\mathrm{i} \omega t} \mathrm{dp}}{\mathrm{dzdy}}=\frac{\frac{A}{\mathrm{LW}}}{l} \mathrm{e}^{-\mathrm{i}(\omega t-k l)}
$$

which is the pressure per length and per width of the aperture. This is reasonable and is really a restatement of a solution to the wave equation for three dimensions [see Dyer, 1989]. The time-varying pressure dp equals a constant, $A /(L W)$, divided by the path distance $l$ (for spherical spreading), and multiplied by a timephase factor. Note that since $\mathrm{e}^{-\mathrm{i} \omega t}$ appears on both sides of (A.1), it may be divided from both sides. This is the so-called removal of time-dependence from the analysis. We also let $d y=W$, since we are confining this study to an xz-plane, and the distance from any $y$-direction side of $\mathrm{dz}$ to the observer point $\mathrm{O}$ is assumed to be close $10 l$. 

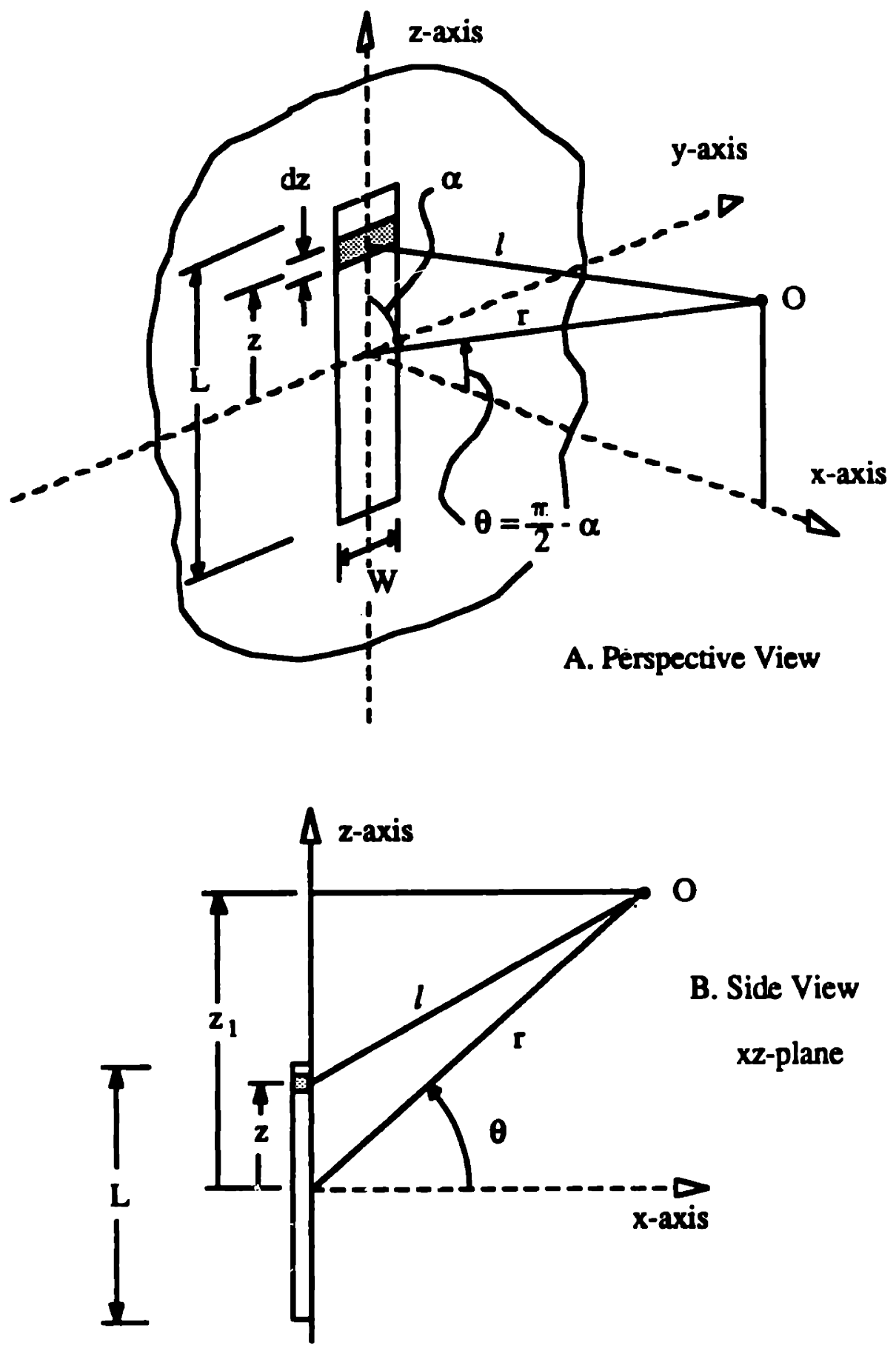

Figure A.1 - Rectangular Aperture 
Making these assumptions, we rewrite (A.1) as

$$
\mathrm{dp}=\frac{(\mathrm{A} / \mathrm{L})}{l} \mathrm{e}^{\mathrm{ikl} l \mathrm{dz}}
$$

To find the total pressure at $O$, we sum the differential pressure over $\mathrm{dz}$, which is nonzero between $+L / 2$ and $-L / 2$, and zero elsewhere (a finite aperture). The total pressure $p$ at point $O$ is written in the form of the integral

$$
\mathrm{p}=\int_{L / 2}^{L / R} \frac{(\mathrm{A} / \mathrm{L})}{l} \mathrm{e}^{\mathrm{ik} l} \mathrm{dz}
$$

where $A$ is a constant (i.e. the source strength),

$L$ is a constant (the aperture length along the $z$-axis).

$l$ is the length from the differential element center to $O$,

$k$ is the wavenumber, $k=\omega / c=2 \pi f / c=2 \pi / \lambda$,

and $d z$ is the length of the differential element along the z-axis.

Both $A$ and $L$ are constants, but the variable $l$ is not. We now must rewrite $l$ as a function of the integration variable z. Figure A.l also shows the xz-plane geometry including $l, r, z$, and $\theta$, and it introduces a new variable $z_{1}$. Recalling trigonometry, we find that

$$
l=\mathrm{r}\left(1-\frac{2 \mathrm{z}}{\mathrm{r}} \sin \theta+\frac{\mathrm{z}^{2}}{\mathrm{r}^{2}}\right)^{1 / 2}
$$

Equation A.3 is of the form

$$
(1+g)^{1 / 2}
$$

where $g=-\frac{2 \mathrm{z}}{\mathrm{r}} \sin \theta+\frac{\mathrm{z}^{2}}{\mathrm{r}^{2}}$,

and $\mathbf{r}>\mathbf{z}$. 
Using the binomial series expansion for (A.4) (see for example, H.-J. Bartsch, Handbook of Mathematical Formulas, Academic Press, NY [1974], page 432], we find that

$$
l \cong r-z \sin \theta+\frac{z^{2}}{r}\left(\frac{1}{2}-\left(\frac{\sin \theta}{2}\right)^{2}\right)
$$

where we have omitted the higher order terms from the binomial series expansion (which will be shortly justified). We check the goodness of (A.5) by noting that as $\theta$ approaches $\pi / 2, \mathrm{r}$ approaches $l+\mathrm{z}$. We may now substitute (A.5) into (A.2) since we now have $l$ as a function of the integration variable $z$. However, before we proceed with this step, we need to make two key assumptions. The first assumption concems the $l$ in the denominator of (A.2). This is the term for the geometric spherical spreading, and we assume that $l$ may be approximated by $r$. So long as $l$ is large compared to the length $L$ of the aperture, then this is a valid approximation. The second assumption concerns the $l$ in the kernel of the integrand $\mathrm{e}^{\mathrm{ik} l}$. This is a phase term. Of the three terms in (A.5), let's suppose we wish to retain the first two, and omit the third part. Since this is a phase term, we would write the third part as

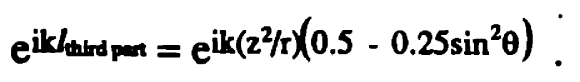

Since this is a phase term, suppose we demand that the phase angle, $k l_{\text {hird }}$ part, be less than $\pi / 4$, or as

$$
\mathrm{k} l_{\text {third part }} \leq \pi / 4 \text {. }
$$

We substitute now for the third part of $l$ :

$$
\mathrm{k}\left(\frac{\mathrm{z}^{2}}{\mathrm{r}}\left(0.5-\frac{\sin ^{2} \theta}{2}\right)\right) \leq \frac{\pi}{4} .
$$

We now substitute $k=\omega / c=2 \pi f / c=2 \pi / \lambda$, and assume the worst-case geometric conditions that $z=L / 2$, and $\theta=0$. By making these substitutions, and solving for $r$, we find the condition on $r$ which allows us to omit the third part of $l$, and therefore omit the higher order terms in $l$. We find that

$$
\mathbf{r} \geq \frac{\mathrm{L}^{2}}{\lambda}
$$

This is called the far-field condition which must be satisfied so that our omission of the third part and higher-order parts of $l$ is valid. Thus $r$ may be held constant as long as (A.9) is satisfied. We now return to 
our expression for pressure, and substitute only the first two terms of $l$ into the kernel of the integrand, and we substitute $l=r$ in the denominator of the integrand:

$$
p=\frac{(A M)}{r} \int_{L / 2}^{L / 2} e^{i k r} e^{-i k z \sin \theta} d z
$$

Since $\mathrm{e}^{\mathrm{ikr}}$ is a phase term that is independent of $\mathrm{z}$, it may be removed from under the integral, and we introduce the variable $s$, where $s=\sin \theta$ :

$$
\begin{aligned}
& p=\frac{A}{L} \frac{e^{i k r}}{r} \int_{L / 2}^{1 / 2} 1 \cdot e^{-i k s z} d z \\
& \text { where } s=\sin \theta .
\end{aligned}
$$

We note (A.11) is an expression which has constants multiplied by an integral on the interval $+L / 2$ to $-L / 2$, of unity, multiplied by $e^{-i k s z}$. We recognize this as the form of a Fourier transform of what we call a rectangle function (the value 1 over the $\mathrm{z}$-interval $+\mathrm{L} / 2$ to $-\mathrm{L} / 2$, and zero elsewhere). Performing the integral, we find the result for the pressure

$$
p=\frac{A}{L} \frac{e^{i k r}}{r} \frac{L \sin \left(\frac{k L}{2} \sin \theta\right)}{\left(\frac{k L}{2} \sin \theta\right)}
$$

We may rewrite the angular portion of (A.12) as a sinc ("sink") function:

$$
\mathrm{p}=\frac{\mathrm{A}}{\mathrm{L}} \frac{\mathrm{e}^{\mathrm{ikg}}}{\mathrm{r}} \mathrm{L} \operatorname{sinc}(\zeta)
$$

where $\operatorname{sinc}(\zeta) \equiv \sin (\zeta / \zeta$

$$
\begin{aligned}
& \zeta=(k L / 2) \sin \theta \\
& k=\omega / c=2 \pi f / c=2 \pi / \lambda
\end{aligned}
$$


We also note that the sinc portion of (A.13) is also called the normalized beam pattem function $B(\theta)$ for an aperture, which we define as

$$
B(\theta) \equiv \frac{\sin \left(\frac{k L}{2} \sin \theta\right)}{\left(\frac{k L}{2} \sin \theta\right)}
$$

- Thus the normalized (the on-axis value is 1.0 ) beam pattern function $B(\theta)$ is therefore a sinc function for a rectangular aperture in one plane (in this analysis, the xz-plane). By the same line of thought shown here, we could find the beam pattern function in the xy-plane, which would have exactly the same form as (A.12) and (A.13) for pressure, and (A.14) for the beam pattern function itself. The only difference would be that the length $L$ would be replaced by the width $W$, and a different angle would used (other than $\theta$ ).

It is important to note that this analysis showed the variation of pressure as a function of angle $(\theta)$ at a distance $r>L^{2} \lambda$ from a rectangular aperture to an observer point $O$. This is a one-way, out-direction, radiated sound result; it would be the sort of measurement taken when finding the beam pattern of a rectangular piston transducer by using a hydrophone at the radial distance $r$, for $r>L^{2} \lambda$. Here, the hydrophone takes the place of the observer at point $O$. The two-way, out- and back-direction result would be when the piston transducer (i.e. the aperture) transmits sound and receives an echo from a small target located at $O$. The two way pattern may be found by the convolution of transmit aperture with the receive aperture, which, in the Fourier transform domain (the wavenumber domain) is the multiplication of the transmit beam pattern with the receive beam pattern. Since the same transducer transmits and receives sound, the aperture is convolved against itself ( a rectangle convolved with a rectangle, which gives a triangle function) and the beam pattern function is $B^{2}(\theta)$, or $\operatorname{sinc}^{2}(\theta)$.

We may also note from (A.14) that $B(\theta)$ is zero (or nulled) when the numerator equals zero. This occurs when the argument of the numerator

$$
\begin{aligned}
& \qquad \frac{\pi \mathrm{L}}{\lambda} \sin (\theta)=\mathrm{n} \pi \\
& \text { and } \mathrm{n}=1,2,3 \text {...etc. }
\end{aligned}
$$

Letting $n=1$, and solving for $\sin (\theta)$ :

$$
\sin (\theta)=\frac{\lambda}{L}
$$


Equation A.16 gives the expression for the angle of the first null in the beam pattern of the L-length rectangular piston. We may then make the small angle approximation that $\sin (\theta) \approx \theta$ :

$$
\theta_{\text {null }} \approx \frac{\lambda}{L}
$$

Since $\theta$ is measured from the main radiation axis, the null-to-null beamwidth is $2 \theta_{\text {null, }}$ or $2 \lambda / \mathrm{L}$. Since we are usually interested in the portion of the full beam width (null-to-null) which contains most of the transmit or reflected energy, we then consider the $-3 \mathrm{~dB}$ beamwidth (the $-3 \mathrm{~dB}$ is the logarithmic halfamplitude value), and this is given by

$$
\theta_{-3 \mathrm{~dB}} \approx \frac{\lambda}{\mathrm{L}}
$$

Equation A.18 is the value usually taken for the beam width of the mainlobe for a rectangular aperture of length $L$, and it represents the beam width from half amplitude to half amplitude ( $-3 \mathrm{~dB}$ to $-3 \mathrm{~dB}$ ) across the span of the main lobe.

\section{Appendix B}

\section{Range Resolution and Pulse Compression}

The resolution of a sonar or radar signal depends on the both the signal transmitted and the type of signal processing available. We may think of resolution as being the smallest distance to which we can resolve two separate objects, or we may think of resolution as the smallest distance to which we may correctly image an object. Figure 2.4 showed how well objects on the bottom could be resolved by a side scan sonar, and gave an example image. This Appendix will review two ways to resolve range: the first is an incoherent means and the second is a coherent means using a replica correlator. In both cases, we ignore the influence of wave dispersion (see Chapter 2.7 in Tolstoy and Clay e.g.)

\section{B.1 Incoherent Range Resolution}

Consider the one-dimensional sonar (or radar) experiment shown in Figure B.1. The transceiver (a transmitter-receiver) is located at the $x$-origin, and transmits a rectangular pulse $s(t)$ at time $t=0$. The 
duration of the pulse is $\tau$. Two identical targets, $A$ and $B$, are also located on the $x$-axis at the respective locations $x=d_{1}$ and $x=d_{2}$, and the targets are assumed to be vanishingly thin along the $x$-direction. The propagation speed for the pulse is $c$. The reflected waves $r(t)$ may be written in the following form:

For the target at $x=d_{1}$,

$$
\begin{aligned}
& \text { Ileading }_{\text {la }}(t)=s\left(t-\frac{2 d_{1}}{c}\right), \\
& r_{\text {trailing }}(t)=s\left(t-\frac{2 d_{1}}{c}-\tau\right),
\end{aligned}
$$

where "leading" and "trailing"are the leading and trailing edges of the pulse $t$. Similarly for the target at $x=$ d2,

$$
\begin{aligned}
& \Pi_{\text {leading }}(t)=s\left(t-\frac{2 d_{2}}{c}\right), \\
& \Gamma_{\text {leading }}(t)=s\left(t-\frac{2 d_{2}}{c}-\tau\right) .
\end{aligned}
$$

Since our desire is to resolve the targets A and B, we need to find the appropriate conditions to do so. To resolve $A$ and $B$, we require that the reflection from the trailing edge of $\operatorname{target} A$ (at $x=d_{1}$ ) occur before the reflection from the leading edge of target $B\left(a t x=d_{2}\right)$. Mathematically, this is written as

$$
t-\frac{2 d_{1}}{c}-\tau<t-\frac{2 d_{2}}{c}
$$

We subtract $t$ from both sides of B.5, and rearrange the terms so that we have the distance between the targets, $d_{2}-d_{1}$, on one side. The result is

$$
\frac{c T}{2}>d_{2}-d_{1}
$$

This shows that the shortest distance between two objects that may be resolved by observing pulses, and not allowing them to overlap, is $c \tau / 2$. We could also have said that the targets $A$ and $B$ were really the leading and trailing edges of a single target $C$, and so we could have only resolved the $x$-length of $C$ to the smallest distance of $\mathrm{c} \tau / 2$. 

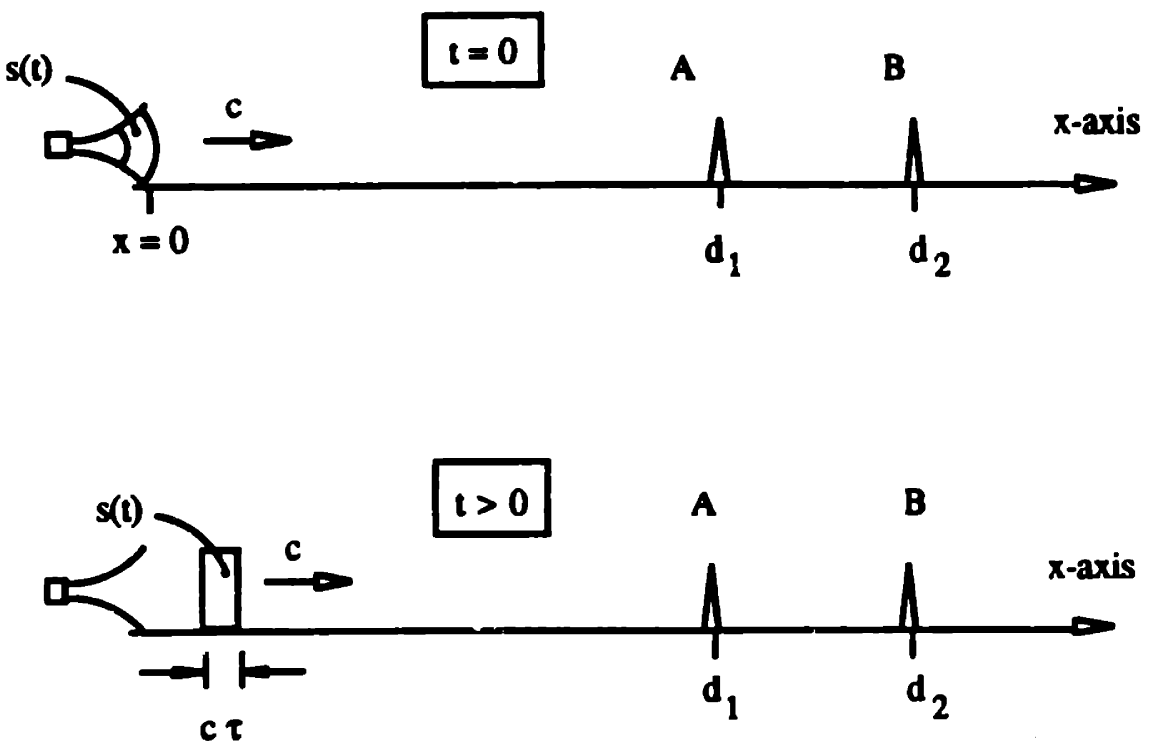

Figure B.1 - One Dimensional View of Range Resolution 


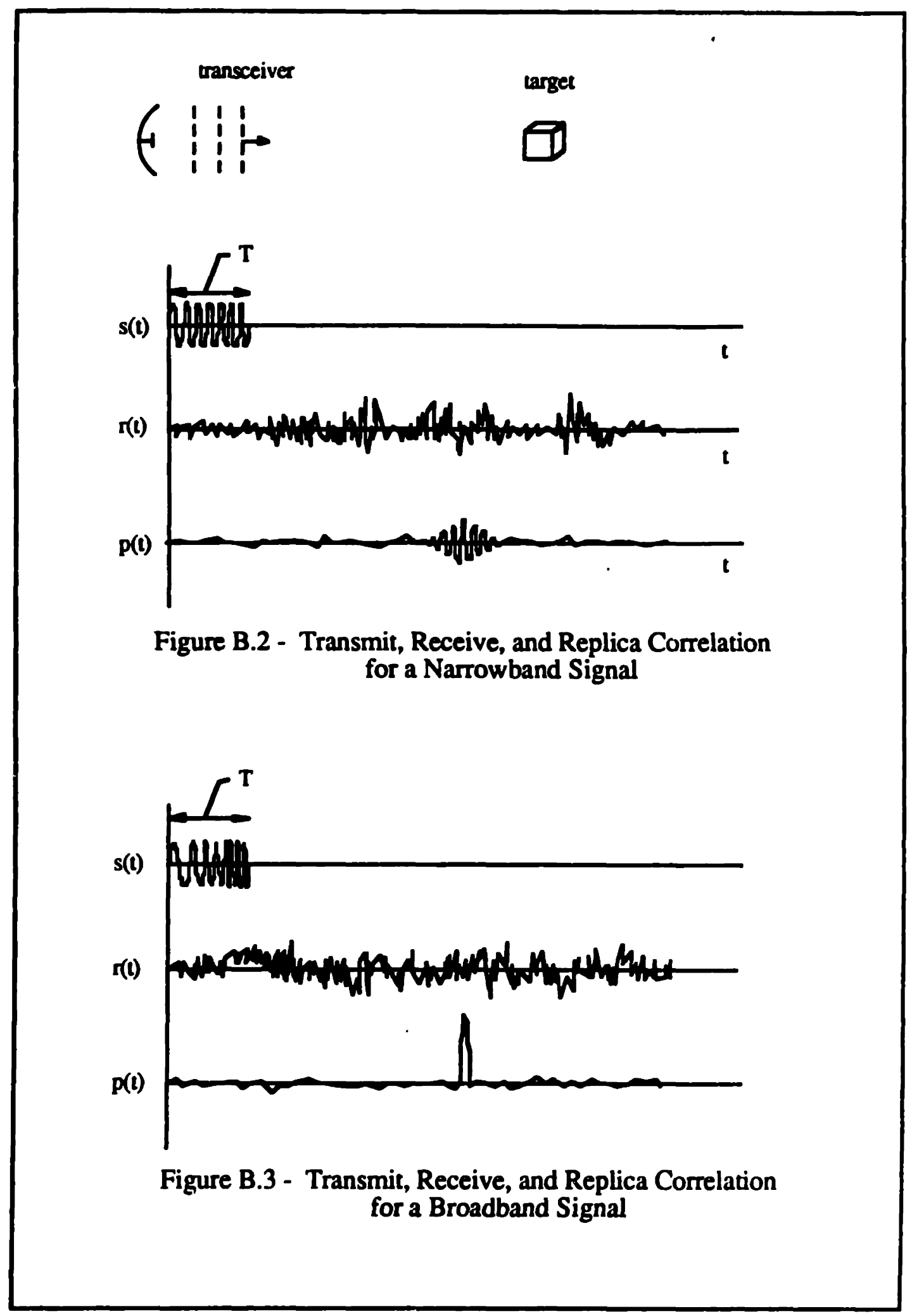


What has just been reviewed here is range resolution formulated from the point of view of a pulse with duration $\tau$, and the observation rule is that the echoes from two distinct targets should not overlap if they are spaced by a distance of greater than $c \tau / 2$. This is one form of range resolution, and it is an incoherent means of detection because it does not rely on the phase of the transmitted signal; instead, it relies only on the envelope of the signal, which was shown here as a rectangular pulse.

\section{B.2 Coherent Range Resolution using Replica Correlation}

Pulse compression is a special name for a form of cross correlation, and equivalently is a form of matched filtering. It is also given the descriptive name replica correlation, because it relies on using a replica of the transmitted signal to find an echo in a received signal, and is therefore unique to active radar and sonar systems.

A signal $s(t)$ is transmitted having time duration $T$, after which a record is made of the received signals as a function of time, or $r(t)$. If we assume that there is only a single stationary target which could return an echo somewhere in $r(t)$, then $r(t)$ will have the same waveform shape as $s(t)$. Geometric spreading and absorption will of course reduce the amplitude of any echo present in $r(t)$, but the waveform will still be preserved and we ignore any dispersive effects.

We need a mathematical way to describe the degree of waveform matching between $r(t)$ and $s(t)$ and so we use the correlator $p(\tau)$ :

$$
p(\tau)=\int_{0}^{t_{\max }} s_{c o p y}(t-\tau) r(t) d t
$$

$$
\begin{array}{ll}
\text { where } & s_{\text {copy }}(t-\tau)=\text { the replica of } s(t) \\
& t_{\max }=\text { the maximum time of received signal. }
\end{array}
$$

Physically this represents the multiply-sum of $s_{\text {copy }}(t)$ with $r(t)$, as $s_{\text {copy }}(t)$ is shifted through the extent of $t$, and it is a mathematical description of the pattern matching. When the waveform $r(t)$ "matches" the waveform $s_{\text {copy }}(t)$, then the value $p(\tau)$ will be large. Similarly where $s_{\text {copy }}(t)$ does not match $r(t)$, the value of $p(\tau)$ will be relatively small. The utility of a wideband $s(t)$ will now be illustrated in the following example. 
Figure B.2 shows a narrowband carrier $f_{0}$, rectangular transmit pulse $s(t)$ having duration $T$. This signal is transmitted to a distant target, and the time series for the returns is shown as $r(t)$. The combination of a weak long-distance scatterer, geometric spreading, and overall system noise makes the identification of the target echo in $r(t)$ impossible. If however, we run $r(t)$ through the replica correlator we

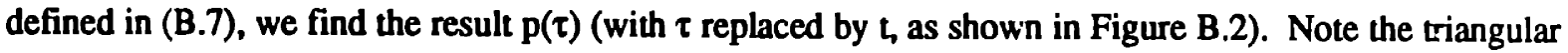
envelope with the sinusoidal carrier which denotes the general vicinity of the target along $t$.

In comparison we show an identical experiment, this time using a wideband transmit signal which is shown as an FM chirp in Figure B.3, and where the bandwidth $B W$ is approximately $f_{2}-f_{1}$. Like the previous example, the received echo in $r(t)$ is too small in amplitude to be observed, but is easily identified in the correlator result $p(t)$. Also note that where we previously had a triangular envelope, we now have an exponential envelope which considerably reduces the level of the sidelobes.

It may be shown that the width of the peak shown in Figure B.3 is $\mathrm{c} /(2 \mathrm{BW})$. Rigorous details on pulse compression, replica correlation, matched filsering, and/or cross correlation may be found in many, many texts in electrical engineering and in radar. A good description may be found in Woodward [1953], as well as in the SAR texts listed at the end of section 1.1.1 and in Siebert [1986]. 


\section{Appendix C}

\section{Program file Examples}

\section{file: COHERE}

This file contains two free-formatted numbers: the first is the coherence time in seconds; the second number is the rms-phase angle in degrees which is a limit for the duration of the coherence time.

This file is read by the program synth.f

This file contains a free-formatted list of ten (the default) depth versus sound-speed points which define a sound speed profile. The last entry is the depth at which the bottom occurs.

$0 . D+0$

8. $5 \mathrm{D}+0$ $1000 . D+0$ $2000 . D+0$ $3000: D+0$ $4000 . D+0$ $5000 . D+0$ $6000 . D+0$ $7000 . D+0$ $8000 . D+0$ 8. $5 D+0$
$1480 . D+0$

$1480.2 \mathrm{D}+0$

$1520 . D+0$

$1520 . D+0$

$1520 . D+0$

$1520 . D+0$

$1520 . D+0$

$1520 . D+0$

$1520 . D+0$

$1520 . D+0$ e.g. at 2000 . meters the sound-speed is $1520 \mathrm{~m} / \mathrm{s}$. 


\section{fi.le name: DECK}

This file contains the input data for the create.out program, which symbolically tabulates sonar echoes without having to store large time series files. The data in the file is free-formatted.

The medium option allows one of 3 options: direct path only (D); direct plus surface-reflected paths (D+SR); or direct plus surface-reflected plus bottom-surface-reflected paths $(D+S R+B S R)$.

Note the use of metric units: meters $(m)$ and meters/second $(\mathrm{m} / \mathrm{s})$. Depth is defined here as a positive quantity, along the z-axis.

The sonar dimensions are given as length (in the horizontal, and parallel to the water surface), vertical (in the depth direction) without any tilt. The last entry for dimensions is for the diameter (if a circular piston is used). The ciameter is ignored if the sonar type is a rectangular piston, and vice versa.

The main radiation axis (mra) of the sonar, or boresight angle in radar, is taken from the horizontal and is in degrees. A down-tilted sonar has a negative mra. No squint angles are allowed (yet).

The file appears below with comments. Don't include the comments in the actual file DECK, just the numbers.

1

1480 .

.517

0.0 .

1

66.5

1.0

$27.625 D+3$

$28.375 D+3$

1.25

$40.0 D-3$

1

300

1

$.3 \quad .050$

$-5.18$

190 . *medium option: 1 for $D, 2$ for $D+S R, 3$ for $D+S R+B S R$

*sound speed at platform depth (m/s)

*platform speed (m/s)

2. *initial platform position $(m): x, y, z$ or range, az, depth * number of target (s)

$65.0 \quad 8.5$ *pos. of target $(s)(m): x, y, z$ or range, az, depth *target scattering sigma

*transmit start frequency (Hz)

*transmit stop frequency ( $\mathrm{Hz}$ )

^pulse repetition freq. or PRF (Hz)

†pulse duration (seconds)

*pulse envelope, 1 for Cos, 2 for triangle, 3 for rect * number of pulses

*sonar type, 1 for rect., 2 for circular piston

.25 *sonar dimensions (m): length, height, diameter

*sonar mra, in degrees from horizontal

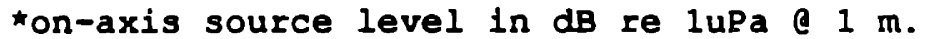


file: GUESS_PROFILE

This file is in the same format as PROEILE

The difference is that PROFILE is the real ocean sound speed profile, whereas GUESS_PROFILE is the estimated profile for the imaging program.

The data is depth (meters), sound speed (m/s) in ten rows and two columns (unformatted), and the 11 th line is the bottom depth.

$\begin{array}{ll}0 . D+0 & 1480 . D+0 \\ 8.5 D+0 & 1480.2 D+0 \\ 1000 . D+0 & 1520 . D+0 \\ 2000 . D+0 & 1520 . D+0 \\ 3000 . D+0 & 1520 . D+0 \\ 4000 . D+0 & 1520 . D+0 \\ 5000 . D+0 & 1520 . D+0 \\ 6000 . D+0 & 1520 . D+0 \\ 7000 . D+0 & 1520 . D+0 \\ 8000 . D+0 & 1520 . D+0 \\ 8.5 D+0 & \end{array}$

file: SAS_CONTROL

This is the file which controls the processing options of the compiled synthetic aperture program synth.out

Below are the control indexes and comments:

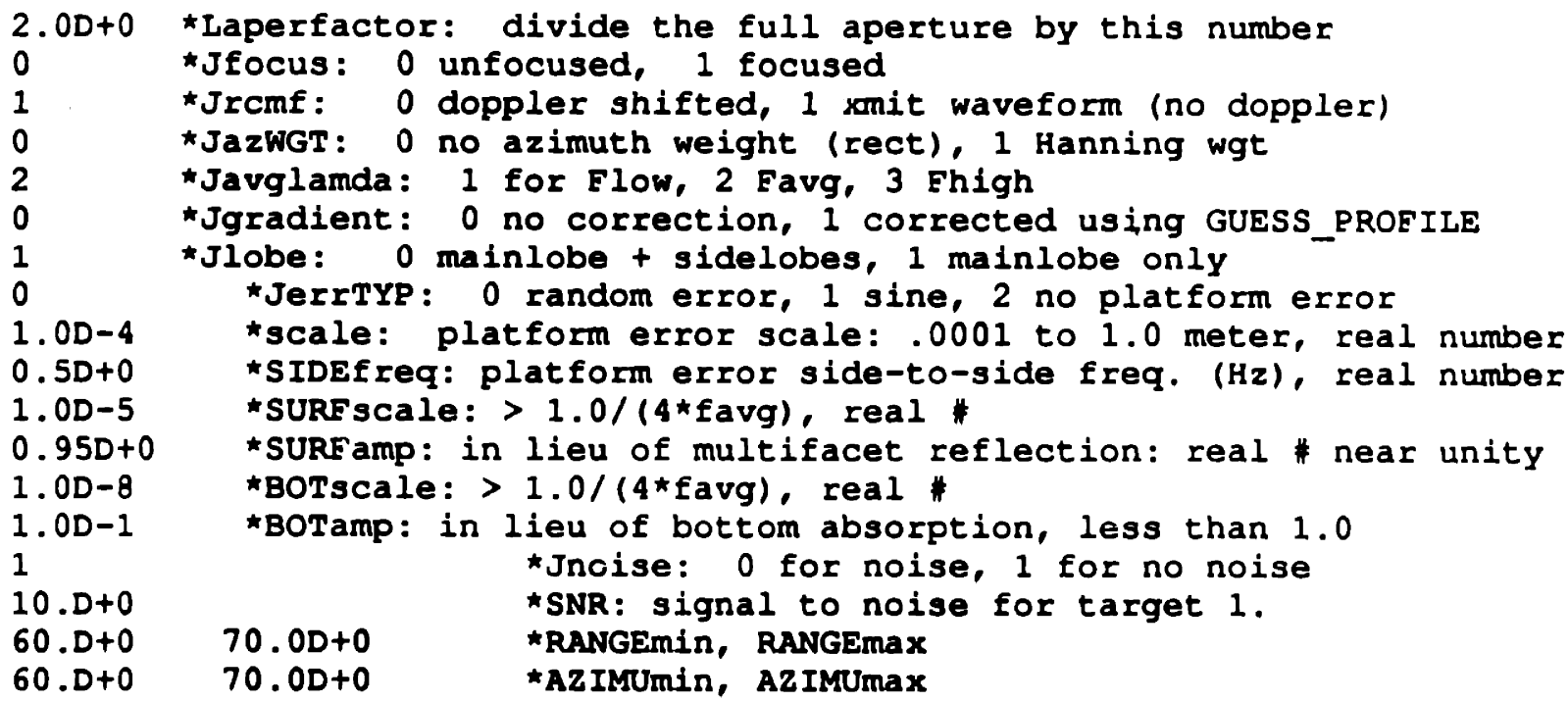




\section{Appendix D}

\section{DOPPLER SHIFT}

The Doppler shift is formulated here for the case of a moving sonar platform and a fixed target. The moving platform/fixed target is the relevant Doppler shift case for the syntheric aperture sonars discussed in this thesis.

\section{Moving Sonar Platform / Fixed Target}

Consider the top view of a moving sonar platform traveling along $y$ in an $x-y$ plane as shown in Figure D.1. The platform has a sonar transceiver on board, and it transmits at frequency $f_{o}$. Meanwhile, a target is located at $x_{0}, y_{0}$ and is, for now, also located in the $x-y$ plane. For illustration purposes, we temporarily make this a 2-D analysis, and later we shall extend it to 3-D geometry.

When the sonar transmits a signal, a stationary observer in the water would perceive either a downshift, no-shift, or up-shift to the frequency tone for observer positions respectively behind, broadside and ahead of the sonar platform transmit position. We call this the outgoing signal into the water and its frequency is given by

$$
f_{\text {out going }}=f_{o}\left(1+\frac{V_{0} \cos \left(\alpha_{0}\right)}{c}\right) \text {. }
$$

$$
\begin{aligned}
& \text { where } \quad V_{0}=\text { the } y \text {-direction platform speed, } \\
& f_{0}=\text { the stable on-board oscillator frequency } \\
& \alpha_{0}=\text { the outgoing angle to the observer measured from forward } \\
& c=\text { the sound speed. }
\end{aligned}
$$

Suppose that the observer were located at $x_{0}, y_{0}$, the target location. The frequency of the sound received would be that given by (D.1). If we assume the target is a perfect reflector such as a comer reflector (as it is known in radar, or a triplane reflector as it has been called in sonar - see Principles and Applications of Underwater Sound, pp 170-174), then an echo will be returned at the same frequency because the target is fixed. We call the frequency of the echo

$$
f_{\text {echo }}=f_{\text {out going }} \text {. }
$$




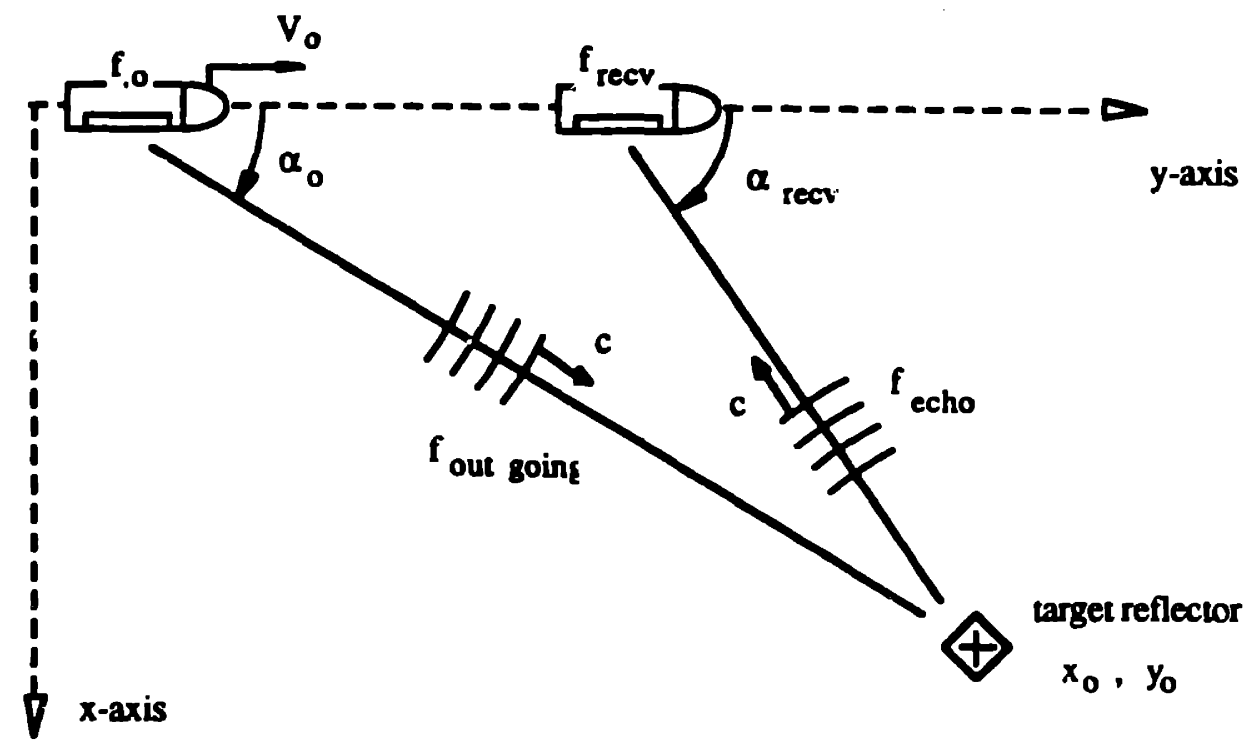

Figure D.1 - 2-D Doppler Geometry

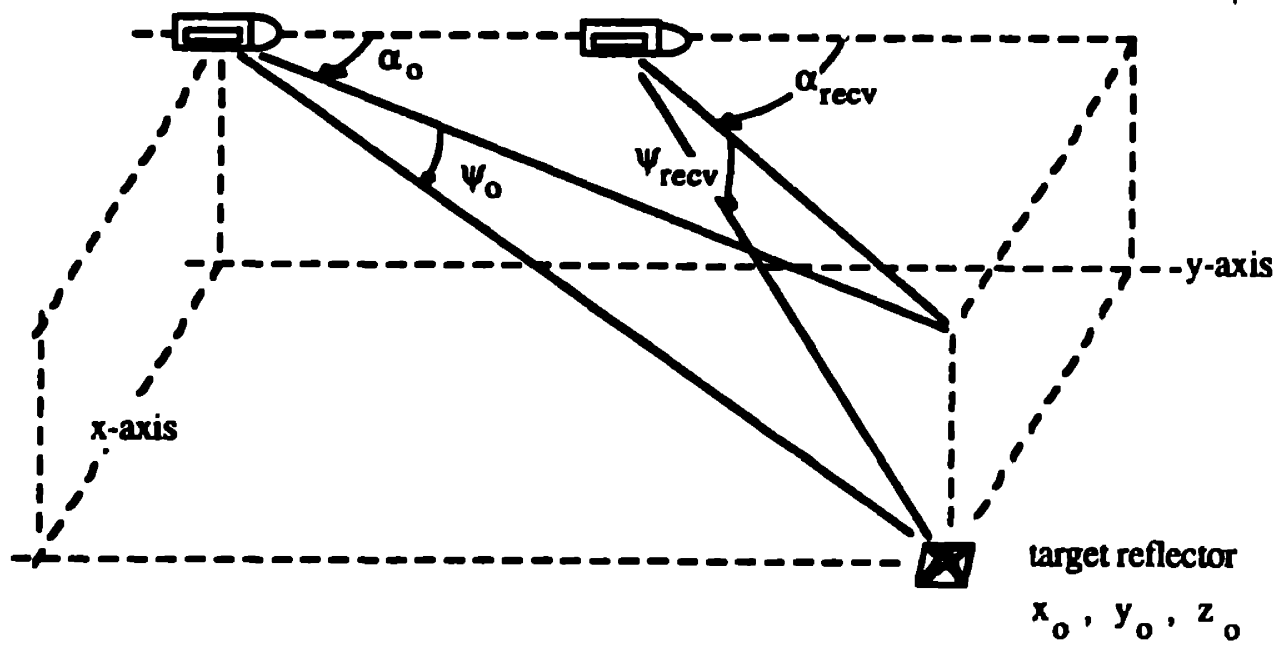

Figure D.2 - 3-D Doppler Geometry 
The sonar meanwhile, has moved to a new position $y+\Delta y$ by the time it receives the echo. Since the sonar is at a new position, it therefore receives the echo from the target a: a different angle than it transmitted. This is given by

$$
f_{\text {recv }}=f_{\text {echo }}\left(1+\frac{V_{o} \cos \left(\alpha_{\text {recv }}\right)}{c}\right)
$$

Combining C.1, C.2 and C.3:

$$
f_{\text {recv }}=f_{o}\left(1+\frac{V_{0} \cos \left(\alpha_{0}\right)}{c}\right)\left(1+\frac{V_{0} \cos \left(\alpha_{\text {recv }}\right)}{c}\right)
$$

Equation D.4 is the form for the Doppler shifted frequency $f_{\text {recv }}$ based on the on-board $f_{0}$ transmitter as influenced by the sonar moving at speed $V_{0}$ and a reflection from a fixed target which was at angle $\alpha_{0}$ for the transmit, and was at an angle of $\alpha_{\text {recv }}$ for the reception. Both angles $\alpha$ are measured with respect to the forward direction $\left(\alpha=0\right.$ ). For example, broadside gives $\alpha=90^{\circ}$.

In most operational instances encountered in practice,

$$
\alpha=\alpha_{0} \approx \alpha_{\text {recv }}
$$

Making this substitution into (D.4):

$$
f_{\text {recv }}=f_{o}\left(1+\frac{2 V_{0} \cos (\alpha)}{c}+\left(\frac{V_{0} \cos (\alpha)}{c}\right)^{2}\right)
$$

For ocean operation $\mathrm{V}_{\mathrm{o}}<15 \mathrm{~m} / \mathrm{s}$ and $\mathrm{c}=1500 \mathrm{~m} / \mathrm{s}$, so $\mathrm{V}_{\mathrm{o}} / \mathrm{c}$ is on the order of .01 (at maximum). If $\cos (\alpha)$ were about 1.0 (a worst-case condition), then the squared term in (D.6) would be at least two orders of magnitude less than the second term in (D.6). For this reason, equation D.6 is often approximated by

$$
\mathrm{f}_{\mathrm{recv}}=\mathrm{f}_{\mathrm{o}}\left(1+\frac{2 \mathrm{~V}_{\mathrm{o}} \cos (\alpha)}{\mathrm{c}}\right)
$$

and $f_{\text {recv }}-f_{0}$ is the so-called Doppler shift.

This is the result for the 2-D case, or where the sonar is close to the bottom and the intended area to be imaged is at a comparatively greater distance. If we extend (D.4) now to the casc where the sonar may not be close to the bottom, or that the target area is not at great range, it may be shown that 


$$
f_{\text {recv }}=f_{0}\left(1+\frac{V_{0} \cos \left(\alpha_{0}\right) \cos \left(\psi_{0}\right)}{c}\right)\left(1+\frac{V_{o} \cos \left(\alpha_{\text {recv }}\right) \cos \left(\psi_{\text {recv }}\right)}{c}\right),
$$

and define the

Doppler factor $\equiv D_{\mathrm{f}}=\left(1+\frac{V_{\mathrm{o}} \cos \left(\alpha_{\mathrm{o}}\right) \cos \left(\psi_{\mathrm{o}}\right)}{\mathrm{c}}\right)\left(1+\frac{\mathrm{V}_{\mathrm{o}} \cos \left(\alpha_{\text {recv }}\right) \cos \left(\psi_{\text {recv }}\right)}{\mathrm{c}}\right)$.

Again, these results may be multiplied out and the higher order terms omitted for the same reasons just shown. The geometry for this expression is shown in Figure D.2.

A few assumptions must now be considered. First, in order for (D.4) to be true, the sonar must continuously transmit (and hence continuously receive). The reason for this is that a continuous sinusoidal wave of frequency $f_{o}$ in the time domain appears as delta functions in the frequency domain. If however, the sonar transmits a finite-length pulse, of say a rectangular envelope over the carrier frequency $f_{0}$, then the frequency domain will show sinc functions centered at $\pm f_{0}$, but having spectral contributions surrounding $f_{o}$ (due to the sinc). Since the real sonar transmits finite duration pulses of frequency $f_{o}$ then there will be spectral spreading, but we assume the Doppler shift is the same for the entire pulse (in time) and so the spectral components will be shifted linearly (i.e. they all have the same Doppler factor).

For a wideband sonar transmitting frequency $f(t)$ then we assume that the Doppler factor (the two terms in parentheses in D.8) are constant with time because the geometry changes little during the transmit duration $\tau$, but the Doppler shift (the Doppler factor multiplied by the frequency) is not constant because $f$ itself changes with time. This shows the distinction between the Doppler factor and the Doppler shift; one is constant over $\tau$, and the other is not.

We also assume that the outgoing angle $\alpha_{0}$, and the incoming or receive angle $\alpha_{\text {recv, }}$ are essentially constant during the pulse duration time $\tau$. By doing this we effectively assume that the platform is moreor-less stationary during transmission and reception. We do not, however, make the assumption that the outgoing angle $\alpha_{0}$ is equal to the receive angle $\alpha_{\text {recv }}$ because synthetic aperture sonars are sensitive to phase by means of the number of wavelengths round-rip from sonar to target and back to sonar. To emulate a real SAS, the create.out program calculates each value separately.

We make the assumption that the geometry changes little during time $\tau$ for transmission or reception on the following grounds and using Figure D.3. For the 2-D case we write the expression for the angle $\alpha$ in D.8 as a function of time in the form of a MacLaurin series: 


$$
\alpha(t)=\alpha(0)+\dot{\alpha}(0) t+\frac{\ddot{\alpha}(0) t^{2}}{2}+\cdots
$$

and so the cosine term in D.8 is given by

$$
\cos (\alpha(t))=\cos \left(\alpha(0)+\dot{\alpha}(0) t+\frac{\ddot{\alpha}(0)}{2} t^{2}+\cdots\right) .
$$

Figure D.3 shows the important geometry features. A single target at $x_{0}, y_{0}$ is shown (where $x_{0}=$ $\mathbf{R}_{\mathbf{0}}$ ), and the platform (as shown by a rectangular aperture sonar) moves along the $y$-axis at $V_{0}, \Delta y$ is the distance from the sonar platform to broadside the target, and the angle from the forward direction to the target is given by $\alpha$. The distance $\Delta y$ as a function of time is given by

$$
\Delta y(t)=y_{0}-V_{0} t,
$$

and trigonometry gives us the relation between $\alpha(t)$ and the change in the geometry:

$$
\tan (\alpha(t))=R_{0} / \Delta y(t)=R_{0} /\left(y_{0}-V_{o} t\right)
$$

Figure D.4 shows $\alpha(t)$ and it's time derivative. The expressions for each are respectively

$$
\begin{aligned}
& \alpha(t)=\frac{\pi}{2}+\tan ^{-1}(t), \\
& \dot{\alpha}(t)=\frac{V_{0}}{R_{0}} \cos ^{2}\left(\alpha-\frac{\pi}{2}\right) .
\end{aligned}
$$

In order to show that the changes in Doppler shift across the transmit pulse due to the change in sonar-target geometry during the transmit pulse time $\tau$, we need to show that $\alpha(t)$ is nearly constant over the duration $\tau$. This means that the $\alpha(t)$ term must be substantially greater than the $\alpha^{\prime}(t)$ and higher terms in equation D.11. Only one operating condition is sufficient to show that the Doppler shift is trivially small over the pulse duration $\tau$, and that occurs when the bearing rate $a^{\prime}(t)$ is a maximum. This occurs when the sonar platform is broadside to the target. In this case, $\alpha(t)$ is $\pi / 2$ while $\alpha^{\prime}(t)$ is $V_{0} / R_{0}$. 


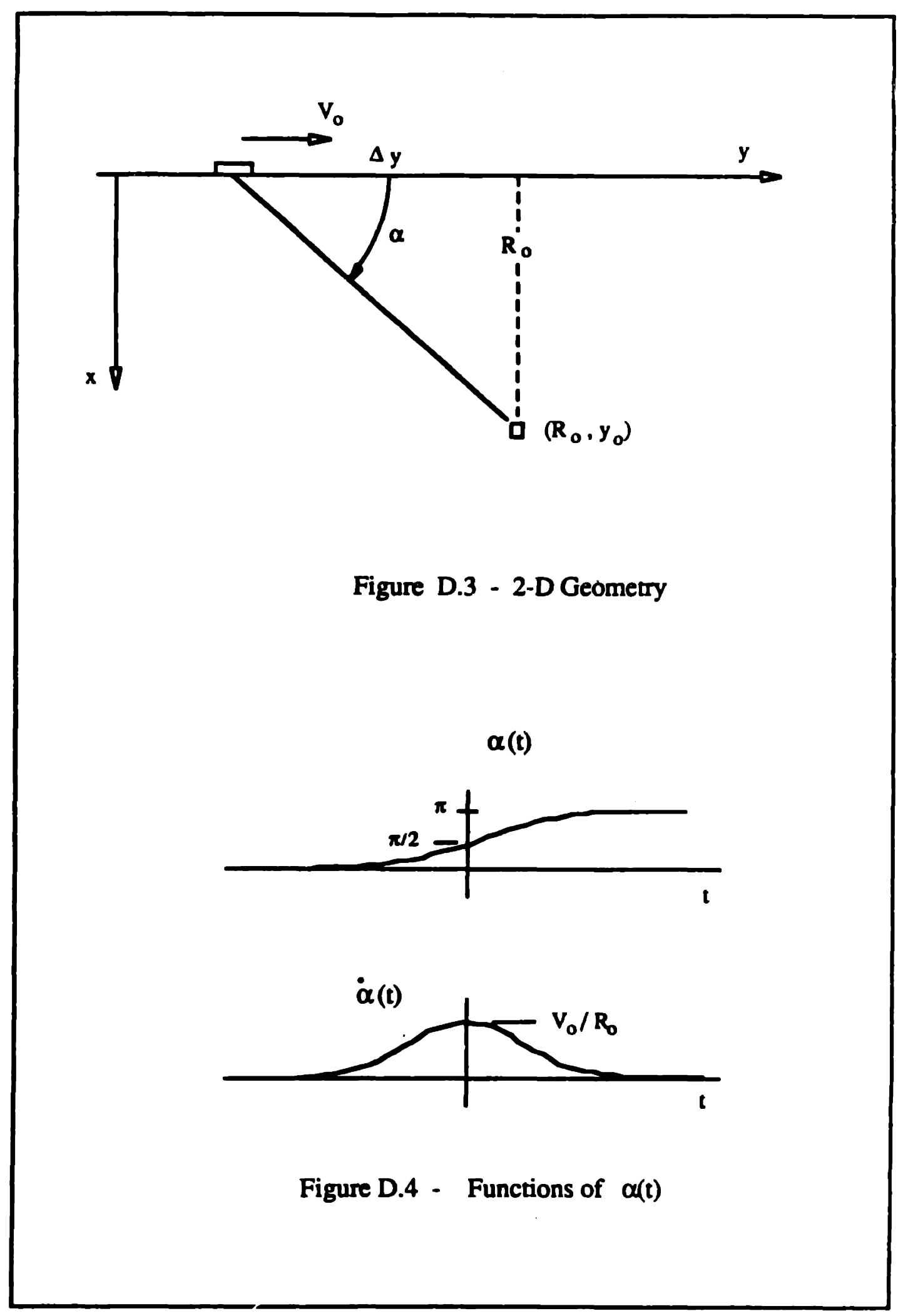


For nearly all the practical cases of concern for synthetic aperture sonar, $V_{0} / R_{0}$ will be substantially smaller than $\pi / 2$, so $\dot{\alpha}(0) t$, or $V_{0} \tau / R_{0}$ will always be much less than $\alpha(t)=\pi / 2$. So the assumption may be made that the angle $\alpha$, for transmit or for receive, is essentially constant over the pulse duration time $\tau$. This also means that the Doppler factor $D_{f}$ is constant over time $\tau$. A more explicit description of this may be found in Rihaczek [1969], section 3.3. These assumptions are used in the computer model.

\section{Appendix E \\ Derive Platform Motion equation}

A platform travels along the $y$-axis at speed $V_{y}$ (note the departure from the usual $V_{0}$ notation). If the platform were absolutely stable, the flight path for the platform would identically be the $y$-axis; however, the motion of most real platforms (in the ocean, air, or in space) is not so ideal. Figure E.1 shows the $y$-axis (the ideal path) and an example actual path whict. is $s(t)$, where $s$ is the displacement in the slant range direction (transverse to $y$ and approximately horizontal). Since the platform travels along $y$ at constant speed, then $s$ is both a function of $y$ and $t$, because $y=V_{y} t$.

Since $s(t)$ is unknown to us, let's treat it in an abstract way by expanding $s(t)$ into a MacLaurin series:

$$
s(t)=s(0)+\frac{s^{\prime}(0)}{1 !} t+\frac{s^{\prime \prime}(0)}{2 !} t^{2} \cdots
$$

We now rewrite (E.1) as

$$
s(t)=S_{0}+V_{0} t+\frac{1}{2} a_{0} t^{2} \cdots
$$

where $S_{0}=s(0), V_{0}=s^{\prime}(0)$, and $a_{0}=s^{\prime \prime}(0)$. We also show $s^{2}(t)$ :

$$
s^{2}(t)=S_{0}^{2}+V_{0}^{2} t^{2}+\frac{1}{4} a_{0}^{2} t^{4}+2 S_{0} V_{o} t+V_{o} a_{0} t^{3}+S_{o} a_{0} t^{2} \cdots
$$

We now take the expectation of $s^{2}(t)$, or $E\left[s^{2}(t)\right]$ or 


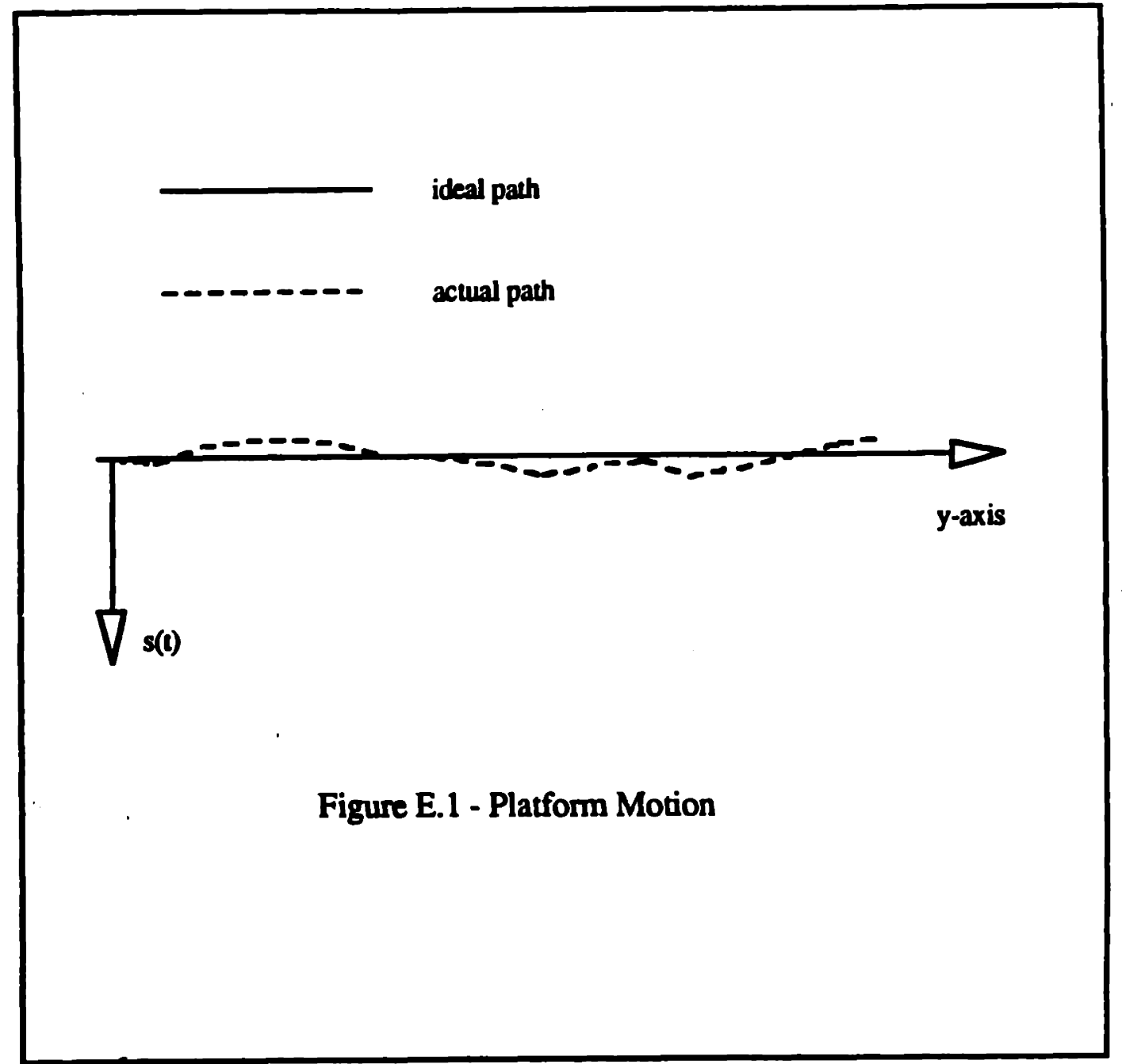




$$
\begin{aligned}
& E\left[s^{2}(t)\right]=\text { variance of } s(t)=\sigma^{2}=\int_{c}^{d} s^{2}(t) p(s) d s \\
& E\left[s^{2}(t)\right]=s_{0}^{2} \int_{c}^{d} p(s) d s+V_{0}^{2} t^{2} \int_{c}^{d} p(s) d s+\frac{a_{0}^{2}}{4} t^{4} \int_{c}^{d} p(s) d s+ \\
& 2 S_{0} V_{0} t \int_{c}^{d} p(s) d s+V_{o} a_{0} t^{3} \int_{c}^{d} p(s) d s+S_{0} a_{0} t^{2} p(s) d s \ldots
\end{aligned}
$$

We also recognize that

$$
\int_{c}^{d} p(s) d s=1.0
$$

for $\mathrm{d}$ and $\mathrm{c}$ being the interval from plus to minus infinity. We also make several assumptions: that $S_{\mathrm{o}}=$ $V_{0}=0$, and we let $a_{0}=\sigma_{\text {acceleration }}=\sigma_{\mathrm{a}}$. Recognizing (E.6) and making these assumptions we find

$$
E\left[s^{2}(t)\right]=\sigma_{\text {position }}^{2}=\sigma_{r}^{2}=\frac{\sigma_{\mathrm{a}}^{2}}{4} \mathfrak{t}^{4}
$$

Equation E.7 is the point at which Cutrona started his analysis on motion compensation considerations (see Cutrona, page 345 [1975] ).

Platform motion errors include displacements in three directions (along-track, cross-track, and heave) and in three rctations (roll, pitch, and yaw), and their derivatives. We have confined our attention to displacements in the cross-track direction, and defer a detailed study of motion compensation to the SAR literature (see Kovaly [1980] and Lozow [1988].) 


\section{Appendix F}

\section{DEFINITIONS}

active

along-track

aperture

antenna

azimuth

B-scan

bistatic

BW

broadband

c.

comelation

cross-track

CTFM

CW

e.g.

et alia

$f_{c}$

FM

hydrophone

i.e.

narrowband

mapping rate

matched filter

$\boldsymbol{N} . \boldsymbol{B}$.

passive

pinger

projector

rade.

SAR

SAS

side looking

side scan

slant range

sonar

squint angle

transducer

wideband as in active sonar. To radiate sound and listen for an echo.

along the same path, or track, as the platform motion.

the area through which radar/sonar/optical energy is transmitted or received.

an aperture.

same as along-track.

brightness scan (see pp. 222-223 in Kino [1987] ).

in transmit from one array, and receive with a second, different array. bandwidth.

a description of a signal where $\mathrm{BW}$ is not $\ll \mathrm{f}_{\mathrm{c}},\left(e . g . \mathrm{BW}>0.1 \mathrm{f}_{\mathrm{c}}\right)$.

( circa); approximately.

the degree of matching or similarity between two things. See Appendix B.

same as slant-range direction. Normal to the along-track direction.

continuous transmission frequency modulation.

continuous wave.

(exempli gratia); Latin: "for example."

or et al., Latin: "and others."

center frequency.

frequency modulation.

an underwater microphone

(id est ); Latin for "that is."

a description of a signal where $B W \ll f_{c}$.

rate of area mapped (by a sonar) per unit time.

see Appendix B.

(Nota Bene ); Latin: "note well."

listen-only radar or sonar operation.

an undenvater sound transmitter used for tracking. They "ping."

sound transmitter; usually describes underwater sound transmitters.

RAdio Detection And Ranging.

Synthetic aperture radar, or synthetic array radar.

synthetic aperture sonar, or synthetic array sonar.

or side-looking; a radar or sonar which operates from the side of its platform.

or side-scan; See side looking.

range from the sonar to a target location on the bottom for an above bottom sonar. SOund Navigation And Ranging.

the angle in the horizontal plane from broadside, either towards forward or aft. as applied to underwater sound, a transducer is a reversible sound energy converter. see broadband. 


\section{BIBLIOGRAPHY}

Autrey, S.A., (1988). "Passive Synthetic Arrays" J. Acoust. Soc. Am. 84(2), 592-598.

Autrey, S.A., (1990). "Reply to "Comments on 'Passive Synthetic Arrays" " [J.Acoust.Soc.Am.85, 1372 (1989)]" J. Acoust. Soc. Am. 87(3), 1355-1356(L) .

Badalyan, V.G. and E.G. Bazulin, (Sept.- Oct., 1989). "The Use of Pseudorandom Sequences in Digital Acoustic Holography" Sov. Phys. Acoust. 35(5), 457-460.

Bennett, S., D.K. Peterson, D. Corl and G.S. Kino, (1982). "A Real-Time Synthetic Aperture Digital Acoustic Imaging System" in Acoustical Imaging, Vol. 10, (P. Alais and A.F. Metherell editors), 669-692 (Plenum Press, NY).

Berktay, H.O., (1985). "Resolution in Sonar Systems - A Review" in Acoustical Imaging, Vol. 14, 69-83 (Plenum Press, NY).

Blacknell, D., and S. Quegan, (1990). "Motion Compensation of Airborne Synthetic Aperture Radars Using Autofocus" GEC Journ. Res. 7(3), 168-182.

Brekhovskikh, L.M., V.V. Krasnoborodko, and V.Ch. Kiriakov, (1987). "Acoustical Visualization of the Ocean Bottom" in Progress in Underwater Acoustics, (H.M. Merklinger, editor), pages 7-13, (Plenum Press, NY. ISBN 0-306-42552-1).

Brown, D.H., (1969). "Application of the Synthetic Aperture Concepts to High Resolution Sonar (U)" in High Resolution Sonar Technology, Vol. II, (National Research Council, Mine Advisory Committee; NRC:MAC:2027, March 1969.) CONFIDENTIAL.

Bucknam, J.N., A.M. Chwastyk, H.D. Black, and F.C. Paddison, (1971). "Synthetic Aperture Sonar" Applied Physics Laboratory/Johns Hopkins University, Tech. Memorandum TG 1161A, May 1971. DTIC number: AD 744657.

Burchìardt, C.B., P. Grandchamp and H. Hoffmann, (1974). "An Experimental 2 MHz Synthetic Aperture Sonar System Intended for Medical Use" IEEE Trans. on Sonics and Ultrasonics SU-21(1), pages 16 (Jan. 1974).

Candy, J.V. and E.J. Sullivan, (1989). "Model-Based Passive Ranging" J. Acoust. Soc. Am. 85(6), 2472-2480.

Carey, W. and N. Yen, (1984). "The Formation of a Synthetic Aperture with Towed Hydrophones" J. Acoust. Soc. Am. 75(S1), S62-63(A).

Carey, W.M., (1988). "Passive Acoustic Synthetic Aperture Processes" lecture given to the Narragansett Chapter of the Acoustical Society of America, 12 January 1988.

Carey, W., (1989). "Comments on 'Passive Synthetic Arrays' [J. Acoust. Soc. Am. 84, 592-598 (1988)]" J. Acoust. Soc. Am. 85(3), 1372(L).

Carey, W.M., (1990). "Reply to Comments by S.W. Autrey [J.Acoust.Soc.Am. 87, 1355-1356 (1990)]" J. Acoust. Soc. Am. 87(3), 1356(L).

Castella, F.R., (1971). "Application of One-Dimensional Holographic Techniques to a Mapping Sonar System" in Acoustical Holography, Vol. 3, (A.F. Metherell, editor), 247-271 (Plenum Press, NY). 
Chramiec, M.A. and G.M. Walsh, (1971). "A Medium Stability Experiment Supporting S، 'AMS, Synthetic Aperture Imaging and Mapping Sonar: An Inspection System for Sea-Bed Arms Control" Raytheon Company, Submarine Signal Division, Portsmouth RI; 31 March 1971.

Christoff, J.T., C.D. Loggins and E.L. Pipkin, (1982). "Measurement of the Temporal Phase Stability of the Medium" J. Acoust. Soc. Am. 71(6), 1606-1607(L).

Cooper, D., (1979). "Synthetic Aperture Sonar Image Processing Study (U)" IBM Federal Systems Division, IBM-79-M74-101, Sept. 1979. CONFIDENTIAL.

Copeland, T.C., (1984). "Bibliography of Underwater Acoustic Imaging and High Frequency, High Resolution Sonars" Naval Coastal Systems Center, Panama City FL, NCSC-TM-392-84; also DTIC number: AD B081337L.

Cutrona, LJ., (1975). "Comparison of Sonar Systems Performance Achievable Using Synthetic-Aperture Techniques vith the Performance Achievable by More Conventional Means" J. Acoust. Soc. Am. 58(2), 336-348.

Cutrona, L.J., (1977). "Additional Characteristics of Synthetic-Aperture Sonar Systems and a Further Comparison with Nonsynthetic-Aperture Sonar Systems" J. Acoust. Soc. Am. 61(5), 1213-1217.

de Heering, P., (1984). "Alternate Schemes in Synthetic Aperture Sonar Processing" IEEE J. Oceanic Eng. OE-9(4), 277-280.

de Heering, P., (1989). Acoustic Synthetic Aperture Processing Theory and Applications, (Dissertation zur erlangen des grades eines Doktors der Ingenieurwissenschaften der Universitat Bremen; PhD. thesis, University of Bremen, Bremen, Germany (formerly FRG]).

Denbigh, P.N., (1977). "Phase Only Side-Scan Sonar for Underwater Mapping" Acoustics Letters, Vol. 1, 84-87.

Dick, M.L., D.E. Dick, F.D. McLeod and N.B. Kindig, (1977). "Ultrasonic Synthetic Aperture Imaging" in Acoustical Holography, (L.W. Kessler, editor), Vol. 7, 327-346 (Plenum Press, NY).

Doctor, S., et alia, (1986). "Real-Time SAFT-UT for Nuclear Component Inspection" in Proceedings of the 8th International Conference on NDE in the Nuclear Industry, (American Society of Metals, Nov. 1986). See also Hall, T.E., L.D. Reid and S.R. Doctor, (no date) "SAFTUT (Synthetic Aperture Technique for Ultrasonic Testing) Real-Time Inspection System: Operational Principles and Implementation" Battelle Pacific Northwest Labs., tech. report PNL-6413, Richland WA. NTIS number: NUREG/CR-5075/HDiM.

Dutkiewicz, M.K. and P.N. Denbigh, (1987). "Synthetic Aperture Sonar for Sub-Bottom Imaging" in Acoustical Imaging, (H.W. Jones, editor), Vol. 15, 585-598 (Plenum Press, NY).

Dyer, I., (1989). "Fundamentals and Applications of Underwater Sound" notes for course 13.851, MIT, Cambridge MA.

Edelson, G.S., and E.J. Sullivan, (1990). "Resolution Performance of the Overlap-Correlator Synthetic Aperture Technique" J. Acoust. Soc. Am. 88(S1), S30(A).

Elachi, C., (1988), Spaceborne Radar Remote Sensing: Applications and Techniques, (ISBN 0-87942-241-6; IEEE Press, NY).

Fay, H.J.W., (c. 1944). Submarine Signal Log, (Submarine Signal Company, Boston MA; reprinted by the Raytheon Company, Submarine Signal Division, Portsmouth RI, Ii( 3). See also the official test account of Capt. J.H. Quinan, Hydrographic Office Bulletin, 13 May' 
Flaherty, J.J., K.R. Erickson and V.M. Lund, (1970). "Synthetic Aperture Ultrasonic Imaging Systems" U.S. Patent 3,548,642; 22 Dec. 1970.

Flemming, B.W. (1982). "A Historical Introduction to Underwater Acoustics with Special Reference to Echo Sounding, Sub-Bottom Profiling and Side Scan Sonar" in Recent Developments in Side Scan Sonar Techniques, (Russell-Cargill, W.G.A. editor, Central Acoustics Laboratory, University of Cape Town, Cape Town, South Africa), pp. 3-9.

Fitch, J.P., (1988). Synthetic Aperture Radar, (ISBN 0-387-96665-X; Springer-Verlag, NY).

Fitzgerald, R.M., A.N. Guthrie, and J.D. Shaffer, (1976). "Low-Frequency Coherence Transverse to the Direction of Propagation" J. Acoust. Soc. Am. 60(3), 752-753(L).

Garmon, L., (1989). "Echoes of War" NOVA (television) broadcast on the story of radar (WGBH, Boston, MA).

Gilmour, G.A., (1978). "Synthetic Aperture Side-Looking Sonar System" U.S. Patent 4,088,978; 9 May 1978. See review in J. Acoust. Soc. Am. 65(2), 557(P) (1979).

Gilmour, G.A., (1980). "High Resolution Sonar" (revised from 1976 edition; Westinghouse Electric Corp., OER No. 76-21).

Gough, P.T., (1986). "A Synthetic Aperture Sonar Capable of Operating at High Speed and in Turbulent Media" IEEE J. Oceanic Eng. OE-11(2), 333-339.

Gough, P.T. and M.P. Hayes, (1989). "Measurement of Acoustic Phase Stability in Loch Linnhe, Scotland" J. Acoust. Soc. Am. 86(2), 837-839(L).

Gough, P.T. and M.P. Hayes, (1989). "Test Results Using a Prototype Synthetic Aperture Sonar" J. Acoust. Soc. Am. 86(6), 2328-2333.

Graham, L.C., (1974). "Synthetic Interferometric Radar for Topographic Mapping" Proc. IEEE 62(6), 763-768. Reprinted in Synthetic Aperture Radar, (J.J. Kovaly, editor. See this bibliography for reference.), 275-280.

Hanish, S., (1975). 'A Review of Synthetic Aperture Sonar', personal communication, (U.S. Naval Research Laboratories, Washington DC).

Hayes, M.P., (1989). A CTFM Synthetic Aperture Sonar, (Ph.D. thesis, University of Canterbury, Dept. of Electrical and Electronic Engineering, Christchurch, New Zealand, September 1989).

Harvey, R., (1990). "Passive Synthetic Aperture Sonar" Conference Book of Proceedings, 1990 Undersea Defence Technology, (Microwave Exhibitions and Publishers Lid, Kent, UK).

Heimiller, R.C., (1962). "Theory and Evaluation of Gain Patterns of Synthetic Arrays" IRE Trans. Mil. Elect. MIL-6(2), 122-129. Reprinted in Synthetic Aperture Radar (see citation for Kovaly, J.J., editor, 1980), 72-79.

Hovanessian, S.A., (1980). Introduction to Synthetic Array and Imaging Radar, (ISBN 089006-082-7; Artech House, Norwood MA).

Hughes, R.G., (1977). "Sonar Imaging with the Synthetic Aperture Method" in Oceans '77 Conf. Record, Vol. 1, pages 10C-1 to 10C-5, (MTS and IEEE publishers; IEEE Publ. number 77CH12724 OEC, 1977).

Ikeda, O., T. Sato, and K. Suzuki (1979). "Super-Resolution Imaging System Using Waves with a Limited Frequency Bandwidth" J. Acoust. Soc. Am. 65(1), 75-81 (1979). 
Ikeda, O., T. Sato, and H. Ohshima (1979). "Synthetic Aperture Sonar in Turbulent Media" J. Acoust. Soc. Am. 66(1), 209-218 (1979).

Ikeda, O., T. Sato (1980). "Further Examination of Synthetic-Apertire Sonar in a Turbulent Medium" J. Acoust. Soc. Am. 68(2), 516-522 (1980). J. Acoust. Soc. Am. 78(1), 112-119 (1985).

Ikeda, O., T. Sato, Y. Minamide and A. Fukushima (1985). "Image Reconstruction from Disturbed Synthetic Aperture Sonar Data Using Aperture Division"

Ikeda, H., et alia, (1988). "Ultrasonic Imaging Apparatus and Method of Forming an Ultrasonic Image of an Object" U.S. Patent 4,793,184; 27 Dec. 1988. Reviewed in J. Acoust. Soc. Am. 86(3), 1212(P) (1989).

Jordan, R.L., (1980). "The Seasat-A Synthetic Aperture Radar System" IEEE J. Oceanic Eng. OE-5(2), 154-164.

Keating, P.N., R.F. Koppelmann, T. Sawatari, and R.F. Steinberg, (1975), "Holographic Aperture Synthesis via a Transmitter Array" in Acoustical Holography, Vol. 6, (N. Booth, editor), 485506 (Plenum Press, NY).

Kino, G.S., (1987). Acoustic Waves: Devices, Imaging, and Analog Signal Processing, (ISBN 0-13-003047-3, Prentice-Hall, NJ).

Klein, M. (1982), in Recent Developments in Side Scan Sonar Techniques, (Russell-Cargill, W.G.A. editor, Central Acoustics Laboratory, University of Cape Town, Cape Town, South Africa).

Kovaly, J.J., editor, (1980). Synthetic Aperture Radar, (ISBN 0-89006-056-8; Artech House, Norwood MA).

Krauss, W. (1967). "Internal Waves" in Underwater Acoustics, Vol. 2, (V.M. Albers, editor), 375-392 (Plenum Press, NY).

Kreuzer, J.L., (1971). "A Synthetic Aperture Coherent Imaging Technique" in Acoustical Holography, Vol. 3, (A.F. Metherell, editor), 287-315 (Plenum Press, NY).

Lee, H.E., (1978). "Synthetic Array Processing for Underwater Mapping Applications" ICASSP 78 (1978 IEEE Int. Conf. on ASSP), 148-151 (IEEE, NY).

Lee, H.E., (1979). "Extension of Synthetic Aperture Radar (SAR) Technique to Undersea Applications" IEEE J. Oceanic Eng. OE-4(2), 60-63.

Liem, R. and T.J. Davis, (1988). "Signal Processing Software for Ground Penetrating Radar User's Manual" Sigma Research, Inc., Redmond WA for the U.S. Naval Civil Engineering Laboratory. DTIC number: AD-A194 286.

Liang, K., B.T. Khuri-Yakub, C-H Chou and G.S. Kino, (1982). "A Three-Dimensional Synthetic Focus System" in Acoustical Imaging, Vol. 10, (P. Alais and A.F. Metherell, editors), 643-668 (Plenum Press, NY).

Loggins, C.D., et alia, (1974). "Synthetic Aperture Sidelooking Sonar (U)" U.S. Navy Journal of Underwater Acoustics 24(4), 457-473 (Oct. 1974). CONFIDENTIAL.

Loggins, C.D., J.T. Christoff, and E.L. Pipkin, (1982). "Results from Rail Synthetic Aperture Experiments" J. Acoust. Soc. Am. 71(S1), S85(A).

Loggins, C.D., (1982). "Synthetic Aperture Sonar - Theory and System Design" J. Acoust. Soc. Am. 72(S1), S73(A). 
Lozow, J., (1986). "Synthetic Aperture Technique for Underwater Acoustic Imaging" Charles Stark Draper Laboratory (memo), (16 Oct. 1986).

Lozow, J., (1988). "INS Motion Compensation Requirements for Synthetic Aperture Sonar" Charles Stark Draper Laboratory, memo JBL-88-129, (2 Aug. 1988).

Macovski, A., (1979). "Ultrasonic Imaging Using Arrays" Proc. IEEE 67(4), 484-495.

McKean, R.S., (1974). "A Preliminary Report on the Acoustic Transmission Experiment at Cobb Seamount" Applied Physics Laboratory/University of Washington, Seattle.

McKinney, C.M. (1982). "An Overview of High-Resolution Sonar Developments" J. Acoust. Soc. Am. 72(S1), S72(A).

Mensa, D.L., (1981). High Resolution Radar Imaging, (ISBN 0-89006-109-2; Artech House, Norwood MA).

MIT, Radiation Laboratory Series. See in particular: L.N. Ridenour, editor, Radar System Engineering. (McGraw-Hill, NY, 1947); and J.S. Hall, editor, Radar Aids to Navigation, (especially Chapter 3, "Characteristics of Airborne Radar"; McGraw-Hill, NY, 1947).

Moshfeghi, M., (1986). "Ultrasound Reflection-Mode Tomography Using Fan-Shaped-Beam Insonification" IEEE Trans. Ultrasonics, Ferroelectrics, and Frequency Control UFFC-33(3), 299314 (May 1986).

Munson, D.C. and R.L. Visentin, (1989); "A Signal Processing View of Strip-Mapping Synthetic Aperture Radar" IEEE Trans. ASSP 37(12), 2131-2147.

NAT Study Group, (1985). "North Atlantic Transect: A Wide-Aperture, Two-Ship Multichannel Seismic Investigation of the Ocean Crust" J. Geophys. Res. 90(B12), pages 10,321 to 10,341.

NCSC, (1989). "A Concept in High Area Coverage Rate Sonar" U.S. Naval Coastal Systems Center (NCSC) Technical Report on Hybrid Synthetic Aperture Sonar, Panama City FL, Dec. 1989.

Nissen, J.M. with A.W. Cockerill, (1989). Winning the Radar War, (ISBN 0-7090-3731-7; R. Hale Lid., London).

Nitadori, K., (1975). "Synthetic Aperture Approach to Multi-Beam Scanning Acoustical Imaging" in Acoustical Holography, Vol. 6, (N. Booth, editor), 507-523 (Plenum Press, NY).

Nitadori, K., K. Mano, and H. Kamata, (198C). "An Experimental Underwater Acoustic Imaging System Using Multi-Beam Scanning" in Acoustical Imaging, Vol. 8, (A.F. Metherell, editor), 249-266 (Plenum Press, NY).

Parkins, B.P. and G.R. Fox, (1971). "Measurement of the Coherence and Fading of Long-Range Acoustic Signals" IEEE Trans. on Audio and Electroacoustics AU-19(2), 158-165 (June 1971).

Peterson, D.K., S.D. Bennett and G.S. Kino, (1982). "Examination of Slots and Cracks with a Real-Time Synthetic Aperture Acoustic Imaging System" J. Acoust. Soc. Am. 71(S1), S81(A).

Preston Jr., K. and J.L. Kreuzer, (1967). "Ultrasonic Imaging Using a Synthetic Holographic Technique" Applied Physics Letters 10(5), 150-152.

Principles and Applications of Underwater Sound, Summary Technical Report of Div. 6 (SubSurface Warfare), Volume 7, Office of Scientific Research and Development, National Defense Research Commituee (Washington, DC, 1946). 
Pritchard, D., (1989). The Radar War: the German Achievement 1904-45, (ISBN 1-85260-2465; Patrick Stephens Ltd., England).

Pusone, E. and L. Lloyd, (1984). "Synthetic Aperture Sonar: An Analysis of Beamforming and System Design" ICASSP 84 (1984 IEEE Int. Conf. on ASSP), Vol. 2, pp. 33.12.1 to 33.12.4, (IEEE, NY).

Pusone, E.G., and L.J. Lloyd, (1985). "Passive Synthetic Aperture Sonar - An Analysis of the Beamforming Process" in Adaptive Methods in Underwater Acoustics (H.G. Urban, editor), NATO ASI Series, Vol. 151, 731-739 (D. Reidel Publishing Company). See also "SyntheticAperture Sonar: Performance Analysis of the Beamforming and System Design" by E.G. Pusone and L.J. Lloyd, SACLANT ASW Research Centre, La Spezia Italy, SACLANTCEN-SR-91, Nov. 1985.

Rihaczek, A.W. (1969). Principles of High-Resolution Radar (McGraw-Hill, NY. Republished by Peninsula Publ., Los Altos, CA).

Rudgers, A., personal communication for synthetic aperture sonar application to acoustic calibration of sources and receivers, U.S. Naval Research Laboratories, Underwater Sound Reference Detachment (USRD), Orlando FL 32856-8337.

Rolt, K., H. Schmidt and J. Milgram, (1990). "Ocean Medium and Platform Effects on Synthetic Aperture Sonar" J. Acoust. Soc. Am. 87(S1), S155(A).

Rolt, K., J. Milgram and H. Schmidt, (1990). "Broadband Undersampled Synthetic Aperture Arrays: Targets Stay Sharp, Aliases Smear" J. Acoust. Soc. Am. 88(S1), S30(A).

Sato, T., M. Ueda and S. Fukuda, (1973). "Synthetic Aperture Sonar" J. Acoust. Soc. Am. 54(3), 799802(L).

Sato, T. and O. Ikeda, (1977a). "Sequential Synthetic Aperture Sonar System - A Prototype of a Synthetic Aperture Sonar System" IEEE Transactions on Sonics and Ultrasonics SU-24(4), 253-259 (July 1977).

Sato, T. and O. Ikeda, (1977b). "Super-Resolution Ultrasonic Imaging by Combined Spectral and Aperture Synthesis" J. Acoust. Soc. Am. 62(2), 341-345 (August 1977).

Sato, T., O. Ikeda, H. Ohshima, and H. Fujikura, (1977c). "A Few Effective Preprocessings in Synthetic Aperture Sonar Systems" in Acoustical Holography, Vol. 7, 569-582 (Plenum Press, NY).

Sato, T., O. Ikeda, and K. Endo (1981). "Combined Spectral and Aperture Synthetic Ultrasonic Imaging System" IEEE Trans. Sonics and Ultrasonics SU-28(2), 64-69 (March, 1981).

Siebert, W. McC., (1986). Circuits, Signals, and Systems, (ISBN: 0-262-19229-2, MIT Press, Cambridge, MA).

Shaffer, J.D., R.M. Fitzgerald and A.N. Guthrie, (1974). "Coherence of Low-Frequency Acoustic Signals in the Deep Ocean" J. Acoust. Soc. Am. 56(4), 1122-1125.

Shishido, M., (1988). "On Synthetic Aperture Sonar" J. Acoust. Soc. Am. 84(S1), S51(A).

Skinner, D.P., and D.W. Ricker, (1980). "Single Pulse Synthetic Aperture Sonar (U)" Applied Research Laboratories - Pennsylvania State University, February 1980. CONFIDENTIAL.

Spiesberger, J.L., R.C. Spindel and K. Metzger, (1980). "Stability and Identification of Ocean Acoustic Multipaths" J. Acoust. Soc. Am. 67(6), 2011-2017.

Spindel, R.C., R.P. Porter, and R.J. Jaffee, (1974). "Long-Range Sound Fluctuations with Drifting Hydrophones" J. Acoust. Soc. Am. 56(2), 440-446. 
Spindel, R.C., (1980). "Multipath processing for Ocean Acoustic Tomography" in EASCON '80 Record, 165-170 (IEEE, NY).

Stergiopoulos, S. and E.J. Sullivan, (1989). "Extended Towed Array Processing by an Overlap Correlator" J. Acoust. Soc. Am. 86(1), 158-171.

Stergiopoulos, S., (1989). "Optimum Bearing Resolution for a Moving Towed Array and Extension of its Physical Aperture" J. Acoust. Soc. Am. 86(S1), S116(A).

Stewart Jr., W.K., (1988). "Multisensor Modeling Underwater with Uncertain Information" Ph.D. thesis, Woods Hole Oceanographic Institution, Woods Hole MA, and Massachusetts Institute of Technology, Cambridge MA, WHOI-89-5, Sept. 1988.

Stowe, D.W., L.H. Wallman, J.W. Follin Jr. and P.J. Luke, (1974). "Stability of the Acoustic Pathlength of the Ocean Deduced from the Medium Stability Experiment" Applied Physics Laboratory/Johns Hopkins University, Tech. Memorandum TG 1230, Jan. 1974. DTIC number: AD 776242.

Stowe, D.W., (1974). (title unknown) (ASW Symposium, Oct. 1974). CONFIDENTIAL.

Stowe, D.W., (1975). (title unknown) U.S. Navy Journal of Underwater Acoustics, (April 1975). CONFIDENTIAL.

Sullivan, E.J. and S. Stergiopoulos, (1989). "A New Method for Passive Synthetic Aperture Array Processing" J. Acoust. Soc. Am. 86(S1), S117(A).

Sutton, J.L., (1979). "Underwater Acoustic Imaging" Proc. IEEE 67, 554-566 (April 1979); reprinted in Modern Acoustical Imaging, (H. Lee and G. Wade, editors; IEEE Press, NY, 1986).

Synthetic Aperture Radar, Technology and Applications (1989), Vols. I and II; and Workshop on SAR Interpretation (1989); both for course 8915 held at the University of Michigan, Engineering Summer Conferences, 17-21 July 1989. A detailed bibliography of SAR and SAR-related books, papers and applications is also included in these volumes.

Tamg, J.H. and C.C. Yang (1987). "Effects of Propagation on the Operation of a Synthetic Aperture Sonar" J. Acoust. Soc. Am. 82(4), 1403-1408.

Thomson, R.N. (1984). "Transverse and Longitudinal Resolution of the Synthetic Aperture Focusing Technique" Ultrasonics 22(1), 9-15 (Jan. 1984).

Thorp, W.H., (1967). "Analytic Description of the Low-Frequency Attenuation Coefficient" J. Acoust. Soc. Am. 42(1), 270(L).

Tolstoy, I., and C.S. Clay, (1987). Ocean Acoustics, Theory and Experiment in Underwater Sound, (ISBN 0-88318-527-X, American Institute of Physics for the Acoustical Society of America, NY, NY).

Tomiyasu, K., (1978). "Tutorial Review of Synthetic-Aperture Radar (SAR) with Applications to Imaging of the Ocean Surface" Proc. IEEE 66(5), 563-583.

Tsujiuchi, J., S. Ueha and K. Ueno, (1974). "Holographic Synthetic Aperture Sonar System" in Ultrasonic Imaging and Holography, (the Proceedings of the U.S.-Japan Science Cooperation Seminar on Pattern Information Processing in Ultrasonic Imaging, Jan. 7-13, 1973; G.W. Stroke, W.E. Kock, Y. Kikuchi, and J. Tsujiuchi editors), 531-552 (Plenum Press, NY).

Urban, H. and S. Stergiopoulos, (1990). "An Experimental Study in Forming a Long Synthetic Aperture in a Nonisotropic Ocean" J. Acoust. Soc. Am. 88(S1), S30(A). 
Urick, R.J., (1982). Sound Propagation in the Sea, (ISBN: 0-932146-08-2, Peninsula Publ, Los Altos, CA).

Walsh, G.M., (1967). "Final Report, Feasibility Study: Synthetic Aperture Array Techniques for High Resolution Ocean Bottom Mapping" Raytheon Company, Submarine Signal Division, Portsmouth RI; Dec. 1967. DTIC number: AD 851498.

Walsh, G.M., (1968). "Final Report, Feasibility Study: Phase 1A, Synthetic Aperture Array Techniques for High Resolutioil Ocean Bottom Mapping" Raytheon Company, Submarine Signal Division, Portsmouth RI; July 1968.

Walsh, G.M., (1969). "Acoustic Mapping Apparatus" U.S. Patent 3,484,737; 16 Dec. 1969. Reviewed in J. Acoust. Soc. Am. 47(5), 1205(P) (1970).

Walsh, G.M. and GJ. Moss, (1970). "A New Approach to Preliminary Site Surveillance" Navigation: J. Inst. Nav. 17(2), 142-148 (Summer 1970).

Werby, M.F., G.J. Tango and H.B. Ali, (1987). "A Synthetic Aperture-Array Technique for Fast Approximate Geobottom Reconnaissance" in Progress in Underwater Acoustics, (H.M. Merklinger, editor), 295-302 (Plenum Press, NY), See also: H.B. Ali and M.F. Werby, (1988), "The Determination of Bottom Layer Thickness in Shallow-Water Ocean Environments Using a Synthetic Aperture Technique" in Acoustics Letters 12(1), 13-17.

Williams, R.E., (1970). "Stability of Ocean Propagated Acoustic Signals" J. Acoust. Soc. Am. 48(1), 92(A).

Williams, R.E. and H.F. Battestin, (1976). "Time Coherence of Acoustics Signals Transmitted Over Resolved Paths in the Deep Ocean" J. Acoust. Soc. Am. 59(2), 312-328. See this article for additional references on medium stability.

Williams, R.E., (1976). "Creating an Acoustic Synthetic Aperture in the Ocean" J. Acoust. Soc. Am. 60(1), 60-73.

Woodward, P.M., (1953). Probability and Information Theory, with Applications to Radar, (Permagon Press Lid., London and McGraw-Hill, NY).

Worcester, P.F., (1981). "An Example of Ocean Acoustic Multipath Identification at Long Range Using Both Travel Time and Vertical Arrival Angle" J. Acoust. Soc. Am. 70(6), 1743-1747.

Yen, N. and W. Carey, (1984). "Application of Synthetic Aperture Processing to Towed Array Data" U.S. Navy Journal of Underwater Acoustics 34(2), 115-146. Reprinted in J. Acoust. Soc. Am. 86(2) 754765 (1989). "DTIC" is the Defense Technical Information Center, Defense Logistics Agency, Cameron
Station, Alexandria VA 22304-6145.

"NTIS" is the National Technical Information Service, Springfield VA 22151. 
